\title{
Embedded contact homology and Seiberg-Witten Floer cohomology II
}

\author{
Clifford Henry TAubes
}

\begin{abstract}
This is the second of five papers that construct an isomorphism between the embedded contact homology and Seiberg-Witten Floer cohomology of a compact 3-manifold with a given contact $1-$ form.
\end{abstract}

57R17; 57R57

\section{Introduction}

This is the second of a five part series whose purpose is to prove that the embedded contact homology of a compact, oriented 3-dimensional manifold with contact 1-form is isomorphic to the manifold's Seiberg-Witten Floer cohomology. This isomorphism theorem is stated formally in the first paper of this series [8]. This part constructs a map between generators of the corresponding complexes that gives the isomorphism, and a map that is used to compare the corresponding differentials. The former map is denoted in [8, Theorem 4.2] by $\Phi^{r}$ and the latter is denoted in [8, Theorem 4.3] by $\Psi^{r}$.

The main theorem here about $\Phi^{r}$ is Theorem 1.1 in Section 1.d. Theorem 1.1 brings together ideas from three distinct parts of geometry. The first part concerns the closed orbits of the Reeb vector field for a given contact structure, the second concerns the moduli spaces of solutions to the vortex equations on $\mathbb{C}$, and the third concerns the moduli spaces of solutions to certain versions of the Seiberg-Witten equations on $M$. Sections 1.a-1.c introduce the necessary background for each of these three topics. The main theorem on $\Psi^{r}$ is Theorem 1.2 in Section 1.g. This theorem brings into play two additional geometric notions. The first is that of a pseudoholomorphic curve in $\mathbb{R} \times M$, and the second is that of a Seiberg-Witten instant on $\mathbb{R} \times M$. Sections 1.e-1.f provide the background material for these last two subjects.

\section{1.a Contact 3-manifolds and Reeb orbits}

This subsection reintroduces from [8] the contact geometry notions that are needed for Theorem 1.1. There are three parts to this reintroduction. 
Part 1 Let $M$ denote the 3-manifold in question and let a denote the contact 1-form. The manifold $M$ is oriented using as volume form $a \wedge d a$. Use $v$ in what follows to denote the Reeb vector field for $a$; this the vector field on $M$ that generates the kernel of $d a$ and pairs with $a$ so as to equal 1. The integral curves of $v$ are deemed to be Reeb orbits. They are oriented implicitly by $v$. The integral of the contact 1 -form along a Reeb orbit $\gamma$ is denoted by $\ell_{\gamma}$.

Fix an almost complex structure, $J$, on the kernel of $a$ so that $d a(\cdot, J(\cdot))$ defines a Riemannian metric on the kernel of $a$. Let $\gamma$ denote a Reeb orbit. This loop in $M$ has a neighborhood that is parameterized by the product of $S^{1}$ and a disk $D \subset \mathbb{C}$ about the origin by an embedding $\varphi: S^{1} \times D \rightarrow M$ which makes $a$, $d a$ and the Reeb vector field $v$ appear as

$$
\begin{aligned}
\frac{2 \pi}{\ell_{\gamma}} a & =\left(1-2 v|z|^{2}-\mu \bar{z}^{2}-\bar{\mu} z^{2}\right) d t+\frac{i}{2}(z d \bar{z}-\bar{z} d z)+\cdots, \\
\frac{2 \pi}{\ell_{\gamma}} d a & =i d z \wedge d \bar{z}-2(v z+\mu \bar{z}) d \bar{z} \wedge d t-2(v \bar{z}+\bar{\mu} z) d z \wedge d t+\cdots, \\
\frac{\ell_{\gamma}}{2 \pi} v & =\frac{\partial}{\partial t}+2 i(v z+\mu \bar{z}) \frac{\partial}{\partial z}-2 i(v \bar{z}+\bar{\mu} z) \frac{\partial}{\partial \bar{z}}+\cdots
\end{aligned}
$$

Here, $v$ and $\mu$ are respectively real and complex valued functions on $S^{1}$. The unwritten terms in the top equation are $O\left(|z|^{3}\right)$ and those in the lower two equations are $O\left(|z|^{2}\right)$. Here and in what follows, the circle $S^{1}$ is implicitly identified with $\mathbb{R} /(2 \pi \mathbb{Z})$ and $t \in \mathbb{R} /(2 \pi \mathbb{Z})$ is used to denote its affine coordinate. These coordinates are such that the vector field $\partial / \partial z$ at $z=0$ pushes forward via $\varphi$ so as to generate the $+i$ eigenspace of $J$ on $\operatorname{kernel}(a)$.

Part 2 Define a first order, $\mathbb{R}$-linear symmetric operator on $C^{\infty}(\mathbb{R} ; \mathbb{C})$, this the operator that takes a function $t \rightarrow z(t)$ to

$$
\mathcal{L} z=\frac{i}{2} \frac{d}{d t} z+v z+\mu \bar{z}
$$

Such an operator is defined given any pair $(\nu, \mu) \in C^{\infty}\left(S^{1} ; \mathbb{R} \oplus \mathbb{C}\right)$. When $z$ is written in terms of real functions $x$ and $y$ as $z=x+i y$, then any function in the kernel of (1-2) can be written as

$$
\left(\begin{array}{l}
x(t) \\
y(t)
\end{array}\right)=U\left(\begin{array}{l}
x(0) \\
y(0)
\end{array}\right) \quad \text { where }\left.U\right|_{t} \in \operatorname{SL}(2 ; \mathbb{R}) \text { for each } t \in \mathbb{R} .
$$

As $t$ varies in $[0,2 \pi]$, the map $\left.t \rightarrow U\right|_{t}$ defines a path in $\operatorname{SL}(2 ; \mathbb{R})$ from the identity. A pair of functions $(\nu, \mu)$ is said to be nondegenerate when the corresponding matrix $U$ has $\operatorname{trace}\left(\left.U\right|_{2 \pi}\right) \neq 2$. The pair is deemed to be elliptic when $\left|\operatorname{trace}\left(\left.U\right|_{2 \pi}\right)\right|<2$ and 
hyperbolic when $\left|\operatorname{trace}\left(\left.U\right|_{2 \pi}\right)\right|>2$. Note that when $(\nu, \mu)$ is hyperbolic, then the $k$-th power of $\left.U\right|_{2 \pi}$ does not have eigenvalue 1 for any $k$. Such is the case because $\left.U\right|_{2 \pi}$ in this case has two real eigenvalues, one with absolute value greater than 1 and the other with absolute value less than 1 . When elliptic, the pair $(\nu, \mu)$ is said to be $n$-elliptic when the $k$-th power of $\left.U\right|_{2 \pi}$ does not have eigenvalue 1 for all $k \leq n$. Note here that a matrix in $\operatorname{SL}(2 ; \mathbb{R})$ whose trace has absolute value less than 2 has two complex eigenvalues, these are on the unit circle and one is the conjugate of the other. A Reeb orbit $\gamma$ is said to be respectively nondegenerate, hyperbolic or $n$-elliptic when such is the case for the functions $(\nu, \mu)$ that come from (1-1). This notion of being hyperbolic or elliptic is intrinsic to $\gamma$; it depends neither on the map $\varphi$ or the almost complex structure $J$.

Let $k$ denote a positive integer. A map from $\mathbb{R}$ to $\mathbb{C}$ is said to be $2 \pi k$-periodic if it is invariant under the shift $t \rightarrow t+2 \pi k$ but is not invariant under any shift $t \rightarrow t+2 \pi k^{\prime}$ for $k^{\prime} \in\{1, \ldots, k\}$. With $S^{1}$ viewed as $\mathbb{R} /(2 \pi \mathbb{Z})$, a map from $S^{1}$ to $C$ is neither more nor less than a $2 \pi$-periodic map from $\mathbb{R}$ to $\mathbb{C}$. The operator $\mathcal{L}$ has trivial kernel on the space of maps from $S^{1}$ to $\mathbb{C}$ if and only if $(\nu, \mu)$ is nondegenerate. If $(\nu, \mu)$ is hyperbolic, then $\mathcal{L}$ has trivial kernel on the space of $2 \pi k$-periodic maps from $\mathbb{R}$ to $\mathbb{C}$ for any positive integer $k$. Meanwhile, if $(\nu, \mu)$ is $n$-elliptic, then $\mathcal{L}$ has trivial kernel on the space of $2 \pi k$-periodic maps from $\mathbb{R}$ to $\mathbb{C}$ for all $k \in\{1,2, \ldots, n\}$.

\section{1.b Vortices and Reeb orbits}

This subsection reintroduces from [8] the spaces of solutions of the vortex equations on $\mathbb{C}$ and discusses some of their relevant features. There are four parts to this subsection.

Part 1 The vortex moduli spaces are labeled by a nonnegative integer, with the integer $n$ version of the vortex moduli space denoted by $\mathfrak{C}_{n}$. The latter consists of certain equivalence classes of pairs $(A, \alpha)$, where $A$ is a Hermitian connection on the trivial complex line bundle over $\mathbb{C}$, and where $\alpha$ is a section of this bundle. A pair $\mathfrak{c}=(A, \alpha)$ is in $\mathfrak{C}_{n}$ if and only if the curvature of $A$ and the $A$-covariant derivative of $\alpha$ satisfy

- $* F_{A}=-i\left(1-|\alpha|^{2}\right)$.

- $\bar{\partial}_{A} \alpha=0$.

- $|\alpha| \leq 1$.

- The function $\left(1-|\alpha|^{2}\right)$ is integrable on $\mathbb{C}$ and $\int_{\mathbb{C}}\left(1-|\alpha|^{2}\right)=2 \pi n$.

Here, $\bar{\partial}_{A}$ denotes the $d$-bar operator that is defined by the connection $A$. The equivalence relation that defines a point in $\mathfrak{C}_{n}$ identifies pairs $(A, \alpha)$ and $\left(A^{\prime}, \alpha^{\prime}\right)$ when $A^{\prime}=A-u^{-1} d u$ and $\alpha^{\prime}=u \alpha$ where $u$ is a smooth map from $\mathbb{C}$ to $S^{1}$. 
Part 2 The vortex moduli space $\mathfrak{C}_{n}$ is a smooth complex manifold, in fact $\mathbb{C}^{n}$. The identification is given by functions $\left\{\sigma_{q}\right\}_{1 \leq q \leq n}$ given by

$$
\sigma_{q}=\frac{1}{2 \pi} \int_{\mathbb{C}} z^{q}\left(1-|\alpha|^{2}\right)
$$

Note that $\sigma_{q}=\sum_{1 \leq k \leq n} z_{j}^{q}$ where $\left\{z_{1}, \ldots, z_{n}\right\}$ denote the set of zeros of $\alpha$ where it is understood that a zero with multiplicity $k$ contributes $k$ identical copies of itself to this set. The function depicted in (1-5) is well defined because

$$
1-|\alpha|^{2}<c_{0} \sum_{1 \leq j \leq m} e^{-\sqrt{2}\left|z-z_{j}\right|},
$$

where $c_{0}$ is a constant that is independent of $n$ and $(A, \alpha) \in \mathfrak{C}_{n}$.

Note that proofs of these and subsequent assertions in this subsection about the solutions to (1-4) either follow directly from what is done by the author in $[4 ; 1]$, or can be established in a straightforward fashion using the techniques in these references. This being the case, the proofs are not given.

The holomorphic tangent space to $\mathfrak{C}_{n}$ at any given $\mathfrak{c}=(A, \alpha)$ is isomorphic to the vector space of square integral pairs, $(x, \iota)$, of complex functions that obey the equations

$$
\partial x+2^{-1 / 2} \bar{\alpha} \iota=0 \quad \text { and } \quad \bar{\partial}_{A} \iota+2^{-1 / 2} \alpha x=0 .
$$

A Hermitian metric on $\mathfrak{C}_{n}$ is defined by declaring the square of the norm of $(x, \iota)$ to be

$$
\frac{1}{\pi} \int_{\mathbb{C}}\left(|x|^{2}+|\iota|^{2}\right) \text {. }
$$

As it turns out this is a complete, Kahler metric. Only in the case $n=1$ is this the metric that is pulled back from $\mathbb{C}^{n}$ using the functions in (1-5). The metric defined by (1-8) and the associated symplectic form are used implicitly in what follows.

Part 3 Let $(\nu, \mu)$ denote a pair consisting of a real number and a complex number. Any such pair defines a function, $h$, on $\mathfrak{C}_{n}$ given by

$$
\hbar=\frac{1}{4 \pi} \int_{\mathbb{C}}\left(2 v|z|^{2}+\left(\mu \bar{z}^{2}+\bar{\mu} z^{2}\right)\right)\left(1-|\alpha|^{2}\right) .
$$

As with any function on $\mathfrak{C}_{n}$, this one defines a Hamiltonian vector field as defined by the symplectic form from the Kahler metric.

Now suppose that $\nu$ and $\mu$ are respectively, a real valued function on $S^{1}$ and a $\mathbb{C}-$ valued function on $S^{1}$. Then (1-9) defines a 1-parameter family of Hamiltonian vector 
fields on $\mathfrak{C}_{n}$. Of interest are the closed, integral curves of this now time dependent vector field. These are maps $\mathfrak{c}: S^{1} \rightarrow \mathfrak{C}_{n}$ that obey at each $t \in S^{1}$ the equation

$$
\frac{i}{2} \mathfrak{c}^{\prime}+\left.\nabla^{(1,0)} \mathfrak{h}\right|_{\mathfrak{c}}=0
$$

where $\mathfrak{c}^{\prime}$ is shorthand for the $(1,0)$ part of $\mathfrak{c}_{*}(d / d t)$, and where $\nabla^{(1,0)} \mathfrak{h}$ denotes the $(1,0)$ part of the gradient of $h$.

A map c: $S^{1} \rightarrow \mathfrak{C}_{n}$ defines the symmetric operator

$$
\zeta \rightarrow \frac{i}{2} \nabla_{t} \zeta+\left.\left(\nabla_{\zeta_{\mathbb{R}}} \nabla^{1,0} \hbar\right)\right|_{c}
$$

on $C^{\infty}\left(S^{1} ; \mathfrak{c}^{*} T_{1,0} \mathfrak{C}_{n}\right)$. Here, $\nabla_{t}$ is the covariant derivative on $C^{\infty}\left(S^{1} ; \mathfrak{c}^{*} T_{1,0} \mathfrak{C}_{n}\right)$ as defined by the pull back of the Levi-Civita connection on $T_{1,0} \mathfrak{C}_{n}$. Meanwhile, $\left.\left(\nabla_{\zeta_{\mathbb{R}}} \nabla^{1,0} \mathfrak{h}\right)\right|_{\mathfrak{c}}$ denotes the covariant derivative at $\mathfrak{c}$ along the vector defined by $\zeta$ in $\left.T \mathfrak{C}_{n}\right|_{\mathfrak{c}}$ of the vector field $\nabla^{1,0} \mathfrak{h} \in C^{\infty}\left(\mathfrak{C}_{n} ; T_{1,0} \mathfrak{C}_{n}\right)$. The operator depicted in (1-11) can rightly be said to be the linearization of (1-10) at the given map $\mathfrak{c}$. A solution to (1-10) is deemed to be nondegenerate when the corresponding version of (1-11) has trivial kernel.

Part 4 Let $\gamma$ denote a Reeb orbit. Fix a tubular neighborhood map for $\gamma$ of the sort described in Section 1.a. Then $\gamma$ has an associated pair $(\nu, \mu)$ for use in (1-9), this the pair that appears in (1-1) and (1-3). Given $\gamma$ and a positive integer $m$, use $\mathfrak{C}_{(\gamma, m)}$ to denote the set of maps $\mathfrak{c}: S^{1} \rightarrow \mathfrak{C}_{m}$ that obey (1-10).

\section{1.c The Seiberg-Witten equations on $M$}

Fix a metric on $T M$ for which $* d a=2 a$ and $|a|=1$. Such a metric induces one on $\operatorname{kernel}(a)$ that can be written as $d a(\cdot, J(\cdot))$ with $J$ an almost complex structure on $\operatorname{kernel}(a)$. Conversely, an almost complex structure $J$ on $\operatorname{kernel}(a)$ with $d a(\cdot, J(\cdot))$ a metric defines a metric on $T M$ with $|a|=1$ and $* d a=2 a$. If $J$ is given, then the metric on $T M$ will be assumed to be defined in this manner.

Let $F \rightarrow M$ denote a $\operatorname{Spin}_{\mathbb{C}}$ structure. This is a principal $U(2)$ bundle lift of the oriented, orthonormal frame bundle of $M$. Use $\mathbb{S}$ to denote the associated $\mathbb{C}^{2}$ bundle $F \times_{U(2)} \mathbb{C}^{2}$. As done in [8, Section 3.a], introduce the Clifford multiplication homomorphism cl: $T^{*} M \rightarrow \operatorname{End}(\mathbb{S})$. This homomorphism is such that $\operatorname{cl}(b)^{\dagger}=-\operatorname{cl}(b)$ and $\operatorname{cl}(b) \operatorname{cl}\left(b^{\prime}\right)=-\operatorname{cl}\left(*\left(b \wedge b^{\prime}\right)\right)-\left\langle b, b^{\prime}\right\rangle$. Here, $\langle$,$\rangle denotes the metric inner product and *$ denotes the associated Hodge star. The endomorphism $\operatorname{cl}(a)$ on $S$ has square -1 and so its eigenspaces in each fiber define a splitting of $\mathbb{S}$ as the orthogonal, direct sum of two complex, Hermitian line bundles. This direct sum is written here as $E \oplus E K^{-1}$ where 
$E \rightarrow M$ and $K \rightarrow M$ are complex line bundles. The convention has $\operatorname{cl}(a)$ act as $i$ on the first summand and $-i$ on the second. The bundle $K^{-1} \rightarrow M$ is isomorphic as an $\mathrm{SO}(2)$ bundle to the kernel of $a$ in $T M$ with the orientation defined by $d a$. The SeibergWitten equations constitute a system of first order, nonlinear partial differential equations for a pair consisting of a connection on $E$ and a section of $\mathbb{S}=E \oplus E K^{-1}$. The versions used here are written below in Part 5 of what follows. Parts 1-4 set the stage.

Part 1 Introduce two related endomorphisms. The first, $\widehat{c}: \mathbb{S} \otimes T^{*} M \rightarrow \mathbb{S}$, is defined so as to send any given decomposable element $\eta \otimes b$ to $\operatorname{cl}(b) \eta$. The second is a quadratic, bundle preserving map from $\mathbb{S}$ to $i T^{*} M$. The image of any given $\eta \in \mathbb{S}$ under the latter map is written in what follows as $\eta^{\dagger} \tau \eta$. It is defined by the rule $\left\langle b, \eta^{\dagger} \tau \eta\right\rangle=\eta^{\dagger} \operatorname{cl}(b) \eta$.

Part 2 The choice of a connection on $\operatorname{det}(\mathbb{S})$ determines, with the metric's Levi-Civita connection, a covariant derivative, $\nabla: C^{\infty}(M ; \mathbb{S}) \rightarrow C^{\infty}\left(M ; \mathbb{S} \otimes T^{*} M\right)$. Composing with $\hat{c}$ gives the Dirac operator. The contact form, $a$, determines an associated canonical

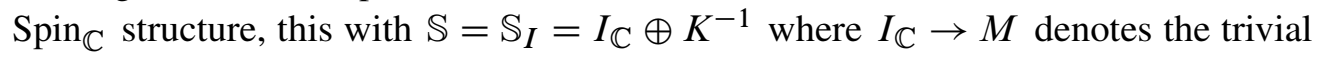
complex line bundle. Fix a unit norm section $1_{\mathbb{C}}$ of $I_{\mathbb{C}}$. This section defines a canonical connection on $K^{-1}=\operatorname{det}\left(\mathbb{S}_{I}\right)$. This is the unique connection for which the section $\psi_{I}=\left(1_{\mathbb{C}}, 0\right)$ of $\mathbb{S}_{I}$ is annihilated by the corresponding Dirac operator. This canonical connection is written as $A_{K}$.

Let $\mathbb{S}=E \oplus E K^{-1}$ now denote the spinor bundle for some other $\operatorname{Spin}_{\mathbb{C}}$ structure. Any given connection on $\operatorname{det}(\mathbb{S})=E^{2} K^{-1}$ can be written as $A_{K}+2 A$ where $A$ is a connection on $E$. The space of smooth connections on $E$ is denoted by $\operatorname{Conn}(E)$.

The symbol $D_{A}$ is used to denote the Dirac operator on $C^{\infty}(M ; \mathbb{S})$ that is defined by using the connection $A_{K}+2 A$ on the line bundle $\operatorname{det}(\mathbb{S})$. This operator sends a given section $\psi$ to $D_{A} \psi=\widehat{c}\left(\nabla_{A} \psi\right)$ where $\nabla_{A}$ denotes the version of $\nabla$ defined by connection $A_{K}+2 A$.

Part 3 Use $B_{A} \in C^{\infty}\left(M ; T^{*} M\right)$ in what follows to denote the Hodge star of the curvature of $A$. This enters in the story through both the Seiberg-Witten equations, and via the functional $\mathrm{E}$ on $\operatorname{Conn}(E)$ that is given by

$$
\mathrm{E}(A)=i \int_{M} a \wedge * B_{A} \text {. }
$$

Part 4 [8, Section 3.d] discusses a certain dense, Banach subspace, $\Omega$, of the Frechet space of smooth, coclosed and exact 1 -forms on $M$. The norm on $\Omega$ is called the "P norm" This $\mathcal{P}$ norm can be assumed to dominate any given $C^{k}$ norm. The versions below of the Seiberg-Witten equations require the choice of a 1 -form from $\Omega$. 
Part 5 Any given $(r, \mu)$ with $r \in[1, \infty)$ and $\mu \in \Omega$ determines a certain version of the Seiberg-Witten equations. These equations require $(A, \psi) \in \operatorname{Conn}(E) \times C^{\infty}(M ; \mathbb{S})$ to obey

$$
\begin{aligned}
& \text { - } B_{A}-r\left(\psi^{\dagger} \tau \psi-i a\right)-i * d \mu+\frac{1}{2} B_{A_{K}}=0 . \\
& \text { - } D_{A} \psi=0 .
\end{aligned}
$$

The group $C^{\infty}(M ; U(1))$ acts on $\operatorname{Conn}(E) \times C^{\infty}(M ; \mathbb{S})$ as follows: If $\mathfrak{c}=(A, \psi)$ and $u \in C^{\infty}(M ; U(1))$, then $u \mathfrak{c}=\left(A-u^{-1} d u, u \psi\right)$. Pairs $\mathfrak{c}$ and $\mathfrak{c}^{\prime}$ from $\operatorname{Conn}(E) \times$ $C^{\infty}(M ; \mathbb{S})$ are said to be gauge equivalent when $\mathfrak{c}^{\prime}=u \mathfrak{c}$ for some $u \in C^{\infty}(M ; U(1))$. The condition that $\mathfrak{c}$ obey (1-13) is preserved by this action of $C^{\infty}(M ; U(1))$. Thus, if ( $A, \psi)$ solves (1-13), then so does $\left(A-u^{-1} d u, u \psi\right)$. With $\mu$ fixed, the set of gauge equivalence classes of solutions to (1-12) is denoted by $M^{r}$.

\section{1.d The map $\Phi^{r}$}

Let $\Theta$ denote a finite set whose typical element is a pair $(\gamma, m)$ whereby $\gamma$ is a Reeb orbit and $m$ is a positive integer. Require in addition that distinct pairs from $\Theta$ have distinct Reeb orbit components, and that the formal sum $\sum_{(\gamma, m) \in \Theta} m \gamma$ defines a chain that represents the Poincare' dual of $c_{1}(E)$ in $H_{1}(M ; \mathbb{Z})$. Let $\mathcal{Z}$ denote the set of such $\Theta$. Given $L \geq 1$, use $\mathcal{Z}^{L} \subset \mathcal{Z}$ to denote the subset $\Theta$ with $\sum_{(\gamma, m) \in \Theta} m \ell_{\gamma}$. An element $\Theta \in \mathcal{Z}$ is said to be nondegenerate when the following is true: If $(\gamma, m) \in \Theta$, then $\gamma$ is nondegenerate, and if elliptic, then also $m$-nondegenerate.

Given $\Theta \in \mathcal{Z}$, let $\mathfrak{C} \Theta$ to denote $\times_{(\gamma, m) \in \Theta} \mathfrak{C}_{(\gamma, m)}$. An element in $\mathfrak{C} \Theta$ associates to each $(\gamma, m) \in \Theta$ a solution to the corresponding version of (1-10). Use $\mathfrak{C} \Theta{ }^{*} \subset \mathfrak{C} \Theta$ to denote the subset with the property that each $\mathfrak{c}_{\gamma}$ is nondegenerate. Fix $L \geq 1$ and introduce $\mathfrak{C} \mathcal{Z}^{L}$ to denote $\left\{\mathfrak{C} \Theta^{*}: \Theta \in \mathcal{Z}^{L}\right\}$ and $\mathfrak{C} \mathcal{Z}^{L *} \subset \mathfrak{C} \mathcal{Z}^{L}$ to denote $\left\{\mathfrak{C} \Theta^{*}: \Theta \in\right.$ $\mathcal{Z}^{L}$ is nondegenerate .

Theorem 1.1 Suppose that $a$ is a contact form on $M$ and that $J$ is an almost complex structure on the kernel of a such that $d a(\cdot, J(\cdot))$ defines a Riemannian metric on the kernel of $a$. Fix $L \geq 1$ and a finite subset $\mathfrak{X} \subset \mathfrak{C Z}^{L *}$. There exists $\kappa>1$ with the following significance: Fix $\mu \in \Omega$ with $\mathcal{P}$ norm less than 1, and fix $r>\kappa$. Use $r$ and $\mu$ to define $M^{r}$. There is an injective map $\Phi^{r}: \mathfrak{X} \rightarrow M^{r}$ whose image lies where $\mathrm{E}<2 \pi L$.

The map $\Phi^{r}$ is constructed in Section 3. The assertions about $\Phi^{r}$ are proved in Section 3.g. 


\section{1.e Pseudoholomorphic subvarieties}

Assume now that the contact form a is such that all elements in $\mathcal{Z}$ are nondegenerate. Such is the case for a residual set in the space contact forms. Fix an almost complex structure, $J$, on $\mathbb{R} \times M$ in the set $\mathcal{J}_{a}$ that is described in [8, Section 2c]. Keep in mind that $J(\partial / \partial s)=v$, that $J$ preserves the kernel of $a$, and that $d a(\cdot, J(\cdot))$ is the induced metric from $M$ on the kernel of $a$. Furthermore, $J$ is translation invariant. A 2-dimensional submanifold $C \subset \mathbb{R} \times M$ is said to be pseudoholomorphic if $J$ maps $T C$ to itself, and if the integral over $C$ of $d a$ is finite.

Let $\Theta$ denote a finite set whose typical element is a pair $(\gamma, m)$ with $\gamma$ a Reeb orbit and with $m$ a positive integer. Assume, as before, that distinct pairs in $\Theta$ have distinct Reeb orbit components. A set such as $\Theta$ appears as a generator of the embedded contact homology chain complex if each hyperbolic Reeb orbit has partner $m=1$. Let $\Theta_{-}$and $\Theta_{+}$denote a given pair of generators of the embedded contact homology chain complex. [8, Section 2.c] assigns to this ordered pair a set $\mathcal{M}_{1}\left(\Theta_{-}, \Theta_{+}\right)$whose typical element, $\Sigma$, is a finite set of the following sort: An element in $\Sigma$ consists of a pair $(C, m)$ where $C$ is a pseudoholomorphic subvariety and where $m$ is a positive integer. In this regard, $m=1$ if $C$ is not an $\mathbb{R}$-invariant cylinder. [8, Section 2.c] describes additional constraints on the elements in $\mathcal{M}_{1}\left(\Theta_{-}, \Theta_{+}\right)$.

As noted in [8], the set $\mathcal{M}_{1}\left(\Theta_{-}, \Theta_{+}\right)$has the structure of a smooth, 1-dimensional manifold. As such, it has a finite set of components, each diffeomorphic to $\mathbb{R}$. In fact, each component is an orbit of the action of $\mathbb{R}$ on the set of pseudoholomorphic subvarieties; this is the action that translates a given subvariety a constant amount along the $\mathbb{R}$ factor of $\mathbb{R} \times M$. Moreover, the identification of a given component with $\mathbb{R}$ can be chosen so as to intertwine the $\mathbb{R}$ action on the set of pseudoholomorphic subvarieties with $\mathbb{R}$ 's action on itself via translation.

\section{1.f Instantons}

A smooth map $\mathfrak{d}: \mathbb{R} \rightarrow \operatorname{Conn}(E) \oplus C^{\infty}(M ; \mathbb{S})$ is deemed to be an instant on when the following conditions are met: Write $\mathfrak{d}=(A, \psi)$. Then

$$
\begin{aligned}
& \text { - } \frac{\partial}{\partial s} A+B_{A}-r\left(\psi^{\dagger} \tau \psi-i a\right)-i * d \mu+\frac{1}{2} B_{A_{K}}=0 . \\
& \text { - } \frac{\partial}{\partial s} \psi+D_{A} \psi=0 .
\end{aligned}
$$

In addition, both the $s \rightarrow-\infty$ and $s \rightarrow \infty$ limits of $\left\{\left.\mathfrak{d}\right|_{s}\right\}_{s \in \mathbb{R}}$ exist, and both limits are solutions to (1-13). Given solutions $\mathfrak{c}_{-}$and $\mathfrak{c}_{+}$to (1-14), use $\mathcal{M}\left(\mathfrak{c}_{-}, \mathfrak{c}_{+}\right)$to denote the set of instantons with $s \rightarrow-\infty$ equal to $\mathfrak{c}_{-}$and with $s \rightarrow \infty$ limit equal to $u \mathfrak{c}_{+}$ with $u \in C^{\infty}(M ; U(1))$. Recall that this set depends only on the gauge equivalence 
classes of $\mathfrak{c}_{-}$and $\mathfrak{c}_{+}$in the following sense: Suppose that $u \in C^{\infty}(M ; U(1))$. If $\mathfrak{d}=(A, \psi) \in \mathcal{M}\left(\mathfrak{c}_{-}, \mathfrak{c}_{+}\right)$, then $u \mathfrak{d}=\left(A-u^{-1} d u, u \psi\right) \in \mathcal{M}\left(u \mathfrak{c}_{-}, \mathfrak{c}_{+}\right)$. The set $\mathcal{M}\left(\mathfrak{c}_{-}, \mathfrak{c}_{+}\right)$enjoys an action of $\mathbb{R}$ that is induced by that of $\mathbb{R}$ on $\mathbb{R} \times M$ via translation along the $\mathbb{R}$ factor.

\section{1.g The map $\Psi^{r}$}

Let $\Theta$ denote a finite set whose typical element is a pair $(\gamma, m)$ with $\gamma$ a Reeb orbit and with $m$ a positive integer. Assume, as always, that distinct pairs in $\Theta$ have distinct Reeb orbit components. Say that $\Theta$ is simple if the following condition is met:

If $\gamma$ is elliptic and paired with an integer $m>1$, then there is a tubular neighborhood of $\gamma$ of the sort described in Section 1.a for which

$$
(\nu, \mu)=\left(\frac{1}{2} \mathrm{R}, 0\right) \quad \text { with } \quad \mathrm{R} \in \mathbb{R} \text { an irrational constant. }
$$

As it turns out, this last assumption implies that $\mathfrak{C} \Theta *=\mathfrak{C} \Theta$; and that this set consists of a single element. This fact is proved in Section 2.b.

Theorem 1.2 Assume that a is such that all elements in $\mathcal{Z}$ are nondegenerate, and that $J \in \mathcal{I}_{a}$. Suppose that both $\Theta_{-}$and $\Theta_{+}$are simple. There exists $\kappa \geq 1$ with the following properties: Fix $\mu \in \Omega$ with $\mathcal{P}$ norm less than 1. Fix $r \geq \kappa$ large enough to define $\Phi^{r}\left(\mathfrak{C}^{r} \Theta_{-}\right)$and $\Phi^{r}\left(\mathfrak{C} \Theta_{+}\right)$via Theorem 1.1. Fix $c_{-}$and $\mathfrak{c}_{+}$in the respective gauge equivalence classes $\Phi^{r}\left(\mathfrak{C} \Theta_{-}\right)$and $\Phi^{r}\left(\mathfrak{C}_{+} \Theta_{+}\right)$. There exists an $\mathbb{R}$-equivariant injective map $\Psi^{r}: \mathcal{M}_{1}\left(\Theta_{-}, \Theta_{+}\right) \rightarrow \mathcal{M}\left(\mathfrak{c}_{-}, \mathfrak{c}_{+}\right)$.

As an addendum to this theorem, note that the construction of $\Psi^{r}$ given below is suitably equivariant with respect to the action of $C^{\infty}(M ; U(1))$. This means the following: Let $u \in C^{\infty}(M ; U(1))$. Then the version of $\Psi^{r}$ that maps into $\mathcal{M}\left(u \mathfrak{c}_{-}, \mathfrak{c}_{+}\right)$is obtained from the version that maps to $\mathcal{M}\left(\mathfrak{c}_{-}, \mathfrak{c}_{+}\right)$by acting by $u$ on the latter map's image.

The construction of $\Psi^{r}$ occupies Sections 4-7 of this article. The assertions about $\Psi^{r}$ are proved in Section 7.k.

\section{1.h Table of contents}

What follows is a table of contents for the remainder of this paper.

Section 2 discusses the vortex equations on $\mathbb{C}$. Solutions to the latter play a prominent role in the construction of both $\Phi^{r}$ and $\Psi^{r}$. The section presents what is needed for these constructions. 
Section 3 constructs the map $\Phi^{r}$. It then proves that $\Phi^{r}$ obeys the assertions that are made by Theorem 1.1.

Section 4 sets the stage for the construction of $\Psi^{r}$. It starts by summarizing what is needed about pseudoholomorphic curves. The constraints on the allowed curves are presented. The section ends with a definition of the parameters that enter the construction.

Section 5 uses the solutions of the vortex equations to construct a set of approximations to the map $\Psi^{r}$. This set is parameterized by a ball in a certain Banach space.

Section 6 explains how each approximate version of $\Psi^{r}$ can be deformed so as to solve most of (1-14).

Section 7 proves that the deformation given in Section 6 solves the whole of (1-14) for one and only one element in Section 5's Banach space. The latter element is used to define the map $\Psi^{r}$. The final subsection here proves Theorem 1.2.

After Section 7 is an index to the notation and then the references. The index to the notation lists the commonly used symbols with the equation number or subsection that contains the definition.

\section{1.i A note on conventions}

What is written below as $c_{0}$ in all cases denotes a constant greater than 1 . The value of this constant can be assumed to increase from appearance to appearance. Unless said otherwise, it should be assumed to be independent of any other constants such as the parameter $r$, or a particular solution to a given equation.

Acknowledgements This work was supported in part by the National Science Foundation.

\section{Vortices on $\mathbb{C}$}

As noted in Section 1.b, the constructions of both $\Phi^{r}$ and $\Psi^{r}$ make use of the solutions to the vortex equations on $\mathbb{C}$. This section constitutes a digression to summarize the salient properties of these solutions. Subsections 2.e-2.g discuss properties that are solely used in the construction of $\Psi^{r}$; the latter can be read just prior to starting Section 4. 


\section{2.a Properties of solutions}

What follows describes the properties of the solutions to (1-4) that are explicitly used in later constructions. The proofs for the properties asserted below either follow directly from [4] and what the author does in [1], and/or are obtained from straightforward modifications of these arguments. This the case, no proofs are given here.

1 Exponential decay There exists for each $k \geq 0$ a constant $c_{k} \geq 1$ with the following significance: Let $\mathfrak{c}=(A, \alpha) \in \mathfrak{C}_{n}$ and let $\mathfrak{Z}=\left\{z_{1}, \ldots, z_{n}\right\} \in \operatorname{Sym}^{n}(\mathbb{C})$ denote the zero locus of $\alpha$. Then

$$
1-|\alpha|^{2}+\sum_{k=1,2, \ldots} c_{k}^{-1}\left|\nabla_{A}^{k} \alpha\right|^{2} \leq c_{0} \sum_{1 \leq j \leq n} e^{-\sqrt{2}\left|z-z_{j}\right|}
$$

at each point $z \in \mathbb{C}$. Here, $\nabla_{A}$ denotes the covariant derivative that is defined by $A$. The following is also true: With $\mathfrak{c}$ and $\mathfrak{Z}$ as above, let $(x, \iota)$ denote a solution to (1-7). Then

$$
\sum_{k=0,1,2, \ldots} c_{k}^{-1}\left(l\left|\nabla^{k} x\right|^{2}+\left|\nabla_{A}^{k} \iota\right|^{2}\right) \leq \sum_{1 \leq j \leq n} e^{-\sqrt{2}\left|z-z_{j}\right|} \int_{\mathbb{C}}\left(|x|^{2}+|\iota|^{2}\right) .
$$

2 The action of $\mathbb{C}$ The action of the group $\mathbb{C}$ on $\mathbb{C}$ by translations induces a holomorphic and isometric action of $\mathbb{C}$ on $\mathfrak{C}_{n}$ because the equations in (1-4) are translationally invariant. This is a free action; its generating vector field at any given $\mathfrak{c}=(A, \alpha)$ is the solution $(x, \iota)$ to $(1-7)$ given by

$$
x=2^{-1 / 2}\left(1-|\alpha|^{2}\right) \quad \text { and } \quad \iota=\partial_{A} \alpha .
$$

Note that this fact has the following two implications: First, the holomorphic identification between $\mathfrak{C}_{1}$ and $\mathbb{C}$ given by the $q=1$ version of (1-5) is, up to a constant, an isometry. Second, the $n>1$ versions of this holomorphic identification between $\mathfrak{C}_{n}$ and $\mathbb{C}^{n}$ is not isometric.

3 The action of $S^{1}$ The group of rotations about the origin in $\mathbb{C}$ also acts isometrically and holomorphically on $\mathfrak{C}_{n}$. This action has a unique fixed point in each $n \geq 1$ version of $\mathfrak{C}_{n}$, this the vortex $(A, \alpha)$ with all $\alpha^{-1}(0)=0$. This fixed point is called the symmetric vortex. It corresponds to the origin in $\mathbb{C}^{n}$ via the holomorphic identification of $\mathfrak{C}_{n}$ with $\mathbb{C}^{n}$ given by the functions $\left\{\sigma_{q}\right\}_{q=1,2 \ldots, n}$ depicted in (1-5). This identification identifies the generator of the $S^{1}$ action with vector field whose type $(1,0)$ component is $\sum_{1 \leq q \leq n} i q \sigma_{q}\left(\partial / \partial \sigma_{q}\right)$. 
4 Localization I The discussion here concerns solutions to (1-4) with the following property: Two or more zeros of $\alpha$ are relatively far apart. To set the stage, fix a set of positive integers $\left\{n_{1}, \ldots, n_{m}\right\}$ that sum to $n$, and then choose a corresponding set $\left\{\mathfrak{c}_{1}, \ldots, \mathfrak{c}_{m}\right\}$ with each $\mathfrak{c}_{j}$ from the corresponding $\mathfrak{C}_{n_{j} 0}$. Write $\mathfrak{c}_{j}=\left(A_{j}, \alpha_{j}\right)$, and let $\mathfrak{Z}_{j} \in \operatorname{Sym}^{n_{j}}(\mathbb{C})$ denote the set of zeros of $\alpha_{j}$. Next, fix a set of distinct points, $z_{1}, \ldots, z_{m}$, in $\mathbb{C}$. Let $\mathfrak{Z}_{j}^{t}$ denote the set obtained from $\mathfrak{Z}_{j}$ by adding $z_{j}$ to each of its elements and let $\mathfrak{c}_{j}^{t}$ denote the corresponding translate of $\mathfrak{c}_{j}$. Write $\mathfrak{c}_{j}^{t}=\left(A_{j}^{t}, \alpha_{j}^{t}\right)$. Thus, $\left(A_{j}^{t}, \alpha_{j}^{t}\right)$ at any given $z \in \mathbb{C}$ is equal to $\left(A_{j}, \alpha_{j}\right)$ at the point $z-z_{j}$.

To continue, define $\mathfrak{c}=(A, \alpha)$ to be the point in $\mathfrak{C}_{n}$ whose corresponding locus in $\operatorname{Sym}^{n}(\mathbb{C})$ is $\mathfrak{Z}_{1}^{t} \cup \cdots \cup \mathfrak{Z}_{m}^{t}$. Then $\alpha=e^{u} \prod_{1 \leq j \leq m} \alpha_{j}^{t}$ where

$$
|\operatorname{Re}(u)| \leq c_{0} \sum_{1 \leq j \neq i \leq m} e^{-\sqrt{2}\left|z_{i}-z_{j}\right|} .
$$

Here, $c_{0}$ is independent of $(A, \alpha)$. In addition, given $k \in\{1,2, \ldots\}$, then the derivatives of the real part of $u$ to order $k$ obey (2-4) as well with $c_{0}$ replaced by a $k$-dependent but $(A, \alpha)$ independent constant.

5 Localization II Let $\mathfrak{c}=(A, \alpha)$ be as just described. The ensuing discussion concerns the vector space, $\mathbb{V}$, of solutions to the corresponding version of (1-7). To this end, let $\mathbb{V}_{j}^{t}$ denote the space of solutions to the $\mathfrak{c}_{j}^{t}$ version of (1-7). This is the translation via the action of $z_{j}$ of the space of solutions to the $\mathfrak{c}_{j}$ version. There exists an isomorphism, $\phi: \bigoplus \mathbb{V}_{j}^{t} \rightarrow \mathbb{V}$ such that if $\varsigma \in \mathbb{V}_{k}^{t}$ has $L^{2}$ norm equal to 1 , then

$$
\begin{aligned}
|\phi(\varsigma)-\varsigma| \leq c_{0}\left(\sum_{1 \leq j \neq i \leq m} e^{-\sqrt{2}\left|z_{i}-z_{j}\right|}\right) e^{-\sqrt{2}\left|z-z_{k}\right|} & \\
& +c_{0} \sum_{1 \leq j \neq k \leq m} e^{-\sqrt{2}\left|z_{k}-z_{j}\right|} e^{-\sqrt{2}\left|z-z_{j}\right|} .
\end{aligned}
$$

In addition, given $k \in\{1,2, \ldots\}$, then the norms of the derivatives $\phi(\varsigma)-\varsigma$ to order $k$ obey (2-5) as well with $c_{0}$ replaced by a $k$-dependent constant.

6 The deformation operator The tangent space to $\mathfrak{C}_{n}$ at any given vortex $\mathfrak{c}=(A, \alpha)$ is characterized in (1-7) as the $L^{2}$ kernel of an operator mapping $C^{\infty}(\mathbb{C} ; \mathbb{C} \oplus \mathbb{C})$ to itself. This operator is denoted by $\vartheta_{\mathfrak{c}}$. Thus

$$
\vartheta_{\mathfrak{c}}(q, \varsigma)=\left(\partial q+2^{-1 / 2} \bar{\alpha}_{\varsigma}, \bar{\partial}_{A} \varsigma+2^{-1 / 2} \alpha q\right) .
$$

It defines a bounded, $\mathbb{C}$-linear Fredholm operator from $L_{1}^{2}(\mathbb{C} ; \mathbb{C} \oplus \mathbb{C})$ to $L^{2}(\mathbb{C}, \mathbb{C} \oplus \mathbb{C})$ with kernel dimension $n$ (over $\mathbb{C}$ ) and with trivial cokernel. Let $\vartheta_{\mathfrak{c}}^{\dagger}$ denote its formal $L^{2}$ adjoint. Note in particular that $\vartheta_{\mathfrak{c}} \vartheta_{\mathfrak{c}}^{\dagger}$ sends any given pair of functions $(q, \varsigma)$ to the pair whose two components are

$$
\frac{1}{4} d^{\dagger} d q+\frac{1}{2}|\alpha|^{2} q \quad \text { and } \quad \frac{1}{2} \nabla_{A}^{\dagger} \nabla_{A} \varsigma+\frac{1}{4}\left(1+|\alpha|^{2}\right) \varsigma .
$$


What with (2-2) and (2-4), this last identity has two consequences: First, the absolute values of the unit $L^{2}$ norm elements in the kernel of $\Theta$ are bounded by $\kappa_{0} e^{-\operatorname{dist}\left(\cdot, \alpha^{-1}(0)\right) / \kappa_{0}}$ with $\kappa_{0}$ independent of $\mathfrak{c}$ and $n$. Second, there exists $\kappa>1$ that is independent of $c$ and such that if $\zeta \in L_{1}^{2}(\mathbb{C} ; \mathbb{C} \oplus \mathbb{C})$ then

$$
\text { - } \int_{\mathbb{C}}\left|\vartheta_{\mathfrak{c}}^{\dagger} \zeta\right|^{2} \geq \kappa^{-1} \int_{\mathbb{C}}\left(\left|\nabla_{\mathfrak{c}} \zeta\right|^{2}+|\zeta|^{2}\right) \text {. }
$$

- $\int_{\mathbb{C}}\left|\vartheta_{\mathfrak{c}} \zeta\right|^{2} \geq \kappa^{-1} \int_{\mathbb{C}}\left(\left|\nabla_{\mathfrak{c}} \zeta\right|^{2}+|\zeta|^{2}\right)$ if $\zeta$ is $L^{2}$-orthogonal to the $L^{2}$-kernel of $\vartheta_{\mathfrak{c}}$.

Here, $\nabla_{\mathfrak{c}}$ is defined by the rule $\nabla_{\mathfrak{c}}(x, \iota)=\left(d x, \nabla_{A} \iota\right)$.

7 Metric properties of $\mathfrak{C}_{n}$ To say more about the metric on $\mathfrak{C}_{n}$, introduce the distance function on $\operatorname{Sym}^{n}(\mathbb{C})$ given by $\operatorname{dist}\left(\mathfrak{Z}, \mathfrak{Z}^{\prime}\right)=\inf _{\sigma \in P(n)} \sum_{1 \leq i \leq n}\left|z_{i}-z_{\sigma(i)}{ }^{\prime}\right|$ with the infimum taken over the set $P(n)$ of permutations of $\{1, \ldots, n\}$. Suppose that $\mathfrak{c}=(A, \alpha)$ and $\mathfrak{c}^{\prime}=\left(A^{\prime}, \alpha^{\prime}\right)$ are points in $\mathfrak{C}_{n}$ and suppose that at least one zero of $\alpha$ has distance 1 or greater from the zero locus of $\alpha^{\prime}$. Then the metric distance between $\mathfrak{c}$ and $\mathfrak{c}^{\prime}$ is such that $c_{0}^{-1} \operatorname{dist}\left(\mathfrak{Z}_{\mathfrak{c}}, \mathfrak{Z}_{\mathfrak{c}^{\prime}}\right) \leq \operatorname{dist}\left(\mathfrak{c}, \mathfrak{c}^{\prime}\right) \leq c_{0} \operatorname{dist}\left(\mathfrak{Z}_{\mathfrak{c}}, \mathfrak{Z}_{\mathfrak{c}^{\prime}}\right)$ where $c_{0} \geq 1$ is independent of $\mathfrak{c}$ and $\mathfrak{c}^{\prime}$. Note that this is a consequence of (2-4) and (2-5).

Here is another consequence of (2-4) and (2-5): There exists $\varepsilon_{0}>0$ with the following significance: Let $\mathfrak{B} \subset T \mathfrak{C}_{n}$ denote the set of vectors with norm less than $\varepsilon_{0}$. Then the exponential map restricts to $\mathfrak{B}$ so as to embed any given fiber by a map whose derivatives to any given order are bounded by a constant that does not depend on the base point.

8 Another exponential map There is a different exponential map from $T \mathfrak{C}_{n}$ to $\mathfrak{C}_{n}$ that is easier to describe. This map is denoted by êxp. Here are its properties: First, there exists $\varepsilon_{0}>0$ such that exp restricts to the radius $\varepsilon_{0}$ ball about the origin in the fiber of $T_{1,0} \mathfrak{C}_{n}$ over any given $\mathfrak{c} \in \mathfrak{C}_{n}$ as an embedding, êxp $\mathfrak{c}$, that sends the origin to $c$. The differential of $\exp _{\mathfrak{c}}$ at the origin is the identity and it agrees with the metric's exponential map to second order. To describe the map exp $\operatorname{ex}_{\mathfrak{c}}$, fix $\zeta \in T_{1,0} \mathfrak{C}_{n}$ with norm less than $\varepsilon_{0}$. Lift $c$ as a pair $(A, \alpha)$ solving (1-4) so as to view $\zeta$ as an element in $\operatorname{kernel}\left(\vartheta_{\mathfrak{c}}\right)$ with $L^{2}$ norm bounded by $\varepsilon_{0}$. Viewed in this way, then $\exp _{\mathfrak{c}}(\zeta)$ can be written as

$$
\operatorname{êxp}_{\mathfrak{c}}(\zeta)=\left(A+2^{-1 / 2}\left(q_{\zeta} d \bar{z}-\bar{q}_{\zeta} d z\right), \alpha+\varsigma \zeta\right),
$$

where $\left(q_{\zeta}, \zeta \zeta\right)=\zeta+\hat{\mathrm{e}}_{\mathfrak{c}}(\zeta)$ and $\hat{\mathrm{e}}_{\mathfrak{c}}(\zeta)$ is defined as follows: First, it is $L^{2}$-orthogonal to the kernel of $\vartheta_{\mathfrak{c}}$ and so can be written as $\vartheta_{\mathfrak{c}}^{\dagger} v_{\zeta}$. Meanwhile, $v_{\zeta} \in C^{\infty}(\mathbb{C} ; \mathbb{C} \oplus \mathbb{C})$ is the unique, small normed solution to the equation

$$
\vartheta_{\mathfrak{c}} \vartheta_{\mathfrak{c}}^{\dagger} v_{\zeta}+2^{-1 / 2}\left(\frac{1}{2} \bar{\zeta} \zeta \sigma_{\zeta}, q_{\zeta} \sigma_{\zeta}\right)=0
$$


This equation for $v_{\zeta}$ guarantees that $\exp _{\mathfrak{c}}(\zeta)$ obeys the equations in (1-4). Moreover, what is written in (2-9) is gauge invariant, so does indeed descend to define a map from $T_{1,0} \mathfrak{C}_{n}$ to $\mathfrak{C}_{n}$.

The inequalities in (2-2) and (2-8) can be used to prove the existence of $\mathfrak{c}$-independent constants $\kappa \geq 1$ and $\varepsilon_{0}>0$ such that the equation for $v_{\zeta}$ has a unique solution with $\sum_{0 \leq k \leq 2}\left\|\nabla^{k} v \zeta\right\|_{2}<\kappa^{-1}$ when $\|\zeta\|_{2} \leq \varepsilon_{0}$. In fact, this norm is bounded by $\kappa\|\zeta\|_{2}^{2}$. Here, the derivative $\nabla$ is defined on an ordered pair $(q, \varsigma) \in C^{\infty}(\mathbb{C} ; \mathbb{C} \oplus \mathbb{C})$ by the rule $\nabla(q, \varsigma)=\left(d q, \nabla_{A} \varsigma\right)$ with $d$ denoting the usual exterior derivative on complex valued functions and $\nabla_{A}$ denoting the covariant derivative that is defined by the connection $A$.

The constant $\varepsilon_{0}$ can be chosen so as to guarantee that the norms of the derivatives of $\hat{e}_{\mathfrak{c}}(\zeta)=\vartheta_{\mathfrak{c}}^{\dagger} v_{\zeta}$ with respect to the coordinate $z$ on $\mathbb{C}$, the element $\zeta \in \operatorname{kernel}\left(\vartheta_{\mathfrak{c}}\right)$ and variations of $c$ in $\mathfrak{C}_{n}$ obey uniform estimates. Indeed, $\varepsilon_{0}>0$ can be chosen so that for each $k \in\{0,1, \ldots\}$, there is a constant $\kappa_{k}$ with the following significance: Fix $\mathfrak{c}=(A, \alpha) \in \mathfrak{C}_{n}$ and let $\left\{z_{j}\right\}_{1 \leq j \leq n} \in \operatorname{Sym}^{n}(\mathbb{C})$ denote the point given by the zeros of $\alpha$. Then

$$
\begin{aligned}
& \text { - }\left|\nabla^{k} \hat{\mathrm{e}}_{\mathfrak{c}}(\zeta)\right| \leq \kappa_{k}\|\zeta\|_{2}^{2} \sum_{1 \leq j \leq n} e^{-\sqrt{2}\left|z-z_{j}\right|} \\
& \text { - }\left|\nabla^{k}\left(\frac{d}{d s} \hat{\mathrm{e}}_{\mathfrak{c}}\left(\zeta+s \zeta^{\prime}\right)\right)\right|_{s=0} \mid \leq \kappa_{k}\|\zeta\|_{2}\left\|\zeta^{\prime}\right\|_{2} \sum_{1 \leq j \leq n} e^{-\sqrt{2}\left|z-z_{j}\right|}
\end{aligned}
$$

To say something about the derivatives of the map $\hat{\mathrm{e}}_{\mathfrak{c}}$ with respect to changes of $\mathfrak{c}$, agree to trivialize $T_{1,0} \mathfrak{C}_{n}$ in a neighborhood of any given $\mathfrak{c} \in \mathfrak{C}_{n}$ using the differential of the map êxp ${ }_{\mathfrak{c}}$. This identifies the kernel of $\vartheta_{\mathfrak{c}}$ with that of $\vartheta_{\mathfrak{c}^{\prime}}$ for $\mathfrak{c}^{\prime}$ near to $\mathfrak{c}$ in $\mathfrak{C}_{n}$. With this identification understood,

$$
\begin{aligned}
& \text { - }\left|\nabla^{k}\left(\frac{d}{d s} \hat{\operatorname{e}}_{\exp _{\mathrm{c}}(s \zeta)}\left(\zeta^{\prime}\right)\right)\right|_{s=0} \mid \leq \kappa_{k}\|\zeta\|_{2}\left\|\zeta^{\prime}\right\|_{2} \sum_{1 \leq j \leq n} e^{-\sqrt{2}\left|z-z_{j}\right|} . \\
& \text { - }\left|\nabla^{k}\left(\frac{d}{d s} \frac{d}{d t} \hat{\mathrm{e}}_{\exp _{\mathrm{c}}(s \zeta)}\left(\zeta^{\prime}+t \zeta^{\prime \prime}\right)\right)\right|_{\substack{s=t=0\\
}} \mid \\
& \quad \leq \kappa_{k}\|\zeta\|_{2}\left\|\zeta^{\prime}\right\|_{2}\left\|\zeta^{\prime \prime}\right\|_{2} \sum_{1 \leq j \leq n} e^{-\sqrt{2}\left|z-z_{j}\right|} .
\end{aligned}
$$

Here, $\kappa_{k}$ is as described in the preceding paragraph.

9 Variation of the kernel of $\vartheta_{\mathfrak{c}}$ Fix $\mathfrak{c} \in \mathfrak{C}_{n}$ and lift $\mathfrak{c}$ to a pair $(A, \alpha)$ solving (1-4). Let $\left.B \subset T^{1,0} \mathfrak{C}_{n}\right|_{\mathfrak{c}}$ denote a ball on which the map $\exp _{\mathfrak{c}}$ is defined. When $\zeta \in B$, use $c_{\zeta}$ to denote $\exp _{\mathfrak{c}}(\zeta)$ as defined in (2-9).

Each $\zeta \in B$ defines a corresponding operator $\vartheta_{c_{\zeta}}$; and the associated $n$-dimensional complex vector subspace $\operatorname{kernel}\left(\vartheta_{\mathfrak{c} \xi}\right) \subset C^{\infty}(\mathbb{C} ; \mathbb{C} \oplus \mathbb{C}) \cap L_{1}^{2}(\mathbb{C} ; \mathbb{C} \oplus \mathbb{C})$. Here, the $L_{1}^{2}$ inner product is defined using the covariant derivative that is defined on a pair $(b, \eta) \in C^{\infty}(\mathbb{C} ; \mathbb{C} \oplus \mathbb{C})$ by $\left(d b, \nabla_{A} \eta\right)$. The point to be made here is that these subspaces 
define a smooth map from $B$ to the Frechet manifold of $n$-dimensional subspaces of $C^{\infty}(\mathbb{C} ; \mathbb{C} \oplus \mathbb{C}) \cap L_{1}^{2}(\mathbb{C} ; \mathbb{C} \oplus \mathbb{C})$. Moreover, the derivatives of this map to any given order at any given $\zeta \in B$ enjoy $c$ independent upper bounds if the radius of $B$ is less than some $\mathfrak{c}$-independent, positive constant. All of this is a consequence of (2-11) and (2-12).

\section{2.b Hamiltonian vector fields on $\mathfrak{C}_{n}$}

This subsection considers solutions to (1-10). With regards to (1-10), note for future reference that the pairing between the differential of $h$ at a given vortex $\mathfrak{c}=(A, \alpha)$ with a $(1,0)$ tangent vector $(x, \iota)$ from $(1-7)$ can be written in a number of ways. What follows are two:

$$
\begin{aligned}
& \text { - }-\frac{1}{\sqrt{2} \pi} \int_{\mathbb{C}}(v \bar{z}+\bar{\mu} z) x . \\
& \text { - } \frac{1}{\pi} \int_{\mathbb{C}}(v \bar{z}+\bar{\mu} z)\left(2^{-1 / 2}\left(1-|\alpha|^{2}\right) x+\bar{\partial}_{A} \bar{\alpha} \iota\right) .
\end{aligned}
$$

Both are derived via integration by parts using (1-7) and (1-4).

There may be many solutions to (1-10) for any given pair of functions $(\nu, \mu)$. However, the story is quite simple in three special cases. What follows is the first.

Lemma 2.1 Suppose that $(v, \mu)$ is nondegenerate. Then the only solution $\mathfrak{c} \rightarrow \mathfrak{C}_{1}$ of the $n=1$ version of (1-10) is the symmetric vortex.

Proof of Lemma 2.1 Use the holomorphic isomorphism between $\mathfrak{C}_{1}$ and $\mathbb{C}$ given by the $q=1$ version of (1-5) to identify these two spaces. Fix any pair of functions $(\nu, \mu)$ on $S^{1}$. With $\mathfrak{C}_{1}$ viewed as $\mathbb{C}$, it follows from (2-13) that the $(\nu, \mu)$ version of (1-10) asks for a map $t \rightarrow z(t)$ from $S^{1}$ to $\mathbb{C}$ that obeys the equation $\mathcal{L} z=0$, where $\mathcal{L}$ is the operator in (1-2). By assumption, the kernel of $\mathcal{L}$ is $\{0\}$.

The next lemma describes the second simple case.

Lemma 2.2 Suppose that $\mathrm{R} \in \mathbb{R}$ is not of the form $p / q$ with $p \in \mathbb{Z}$ and $q \in\{1, \ldots, n\}$. Then the symmetric vortex is the unique solution in $\mathfrak{C}_{n}$ to the version of (1-10) that is defined by the pair $\left(v=\frac{1}{2} \mathrm{R}, \mu=0\right)$.

Proof of Lemma 2.2 It follows by using (2-13) that the Hamiltonian vector field in this case is R times the generator of the $S^{1}$ action on $\mathfrak{C}_{n}$. To see its integral curves, view $\mathfrak{C}_{n}$ as $\mathbb{C}^{n}$ using the holomorphic isomorphism provided by the functions $\left\{\sigma_{q}\right\}_{1 \leq q \leq n}$ that are depicted in (1-5). The integral curve of $\mathrm{R}$ times the generator through a point in 
$\mathbb{C}^{n}$ with coordinates $\left\{\sigma_{q}=a_{q}\right\}_{q=1, \ldots, n}$ is given by the map from $\mathbb{R}$ to $\mathbb{C}^{n}$ that sends $t \in \mathbb{R}$ to the point with coordinates $\left\{\sigma_{q}=e^{i \mathrm{R} q t} a_{q}\right\}_{q=1,2, \ldots, n}$. Since no $q \in\{1, \ldots, n\}$ version of $q \mathrm{R}$ is an integer, the only such curve that descends to map $S^{1}=\mathbb{R} / 2 \pi \mathbb{Z}$ into $\mathfrak{C}_{n}$ as all $a_{q}=0$.

What follows is the final case.

Lemma 2.3 Suppose that $k \in \mathbb{Z}$, that $\varepsilon \in \mathbb{R}-\{0\}$. Set $v=\frac{1}{4} k$ and $\mu=i \varepsilon e^{i k t}$. Then the symmetric vortex is the unique solution in $\mathfrak{C}_{1}$ to the corresponding version of (1-10). Meanwhile, there are no solutions to this version of (1-10) when $n \geq 1$.

Proof of Lemma 2.3 It follows from Lemma 2.1 that the only solution in the case $n=1$ when $\left(v=\frac{1}{4} k, \mu=i \varepsilon e^{i k t}\right)$ is the symmetric vortex. Indeed, such is the case by virtue of the fact that the corresponding matrix $U_{t=2 \pi}$ that appears in (1-3) is hyperbolic.

Now consider the assertion of the lemma for $n>1$. Use the functions $\left\{\sigma_{q}\right\}_{1 \leq q \leq n}$ to again identify $\mathfrak{C}_{n}$ with $\mathbb{C}^{n}$. Write the Kähler metric as $g_{i} \bar{j}_{j} d \sigma_{i} d \bar{\sigma}_{j}$. It is a consequence of (2-13) that the 1-parameter family of diffeomorphisms that is generated by the Hamiltonian vector field defined by the function $h$ in (1-9) is such that the function $t \rightarrow \sigma_{2}(t)$ obeys the equation

$$
\frac{i}{2} \frac{d}{d t} \sigma_{2}+2 v \sigma_{2}+\mu g^{2 \overline{2}}=0 .
$$

Here, $g^{2 \overline{2}}$ comes from the inverse to the Hermitian form that defines the Kahler metric. Note that this is a strictly positive function on $\mathbb{C}^{n}$. In the case at hand, (2-14) reads

$$
\frac{i}{2} \frac{d}{d t} \sigma_{2}+\frac{1}{2} k \sigma_{2}+i \varepsilon e^{i k t} g^{2 \overline{2}}=0 .
$$

To proceed, write the 1-parameter family of diffeomorphisms $t \rightarrow\left\{\sigma_{q}(t)\right\}_{q=1,2, \ldots, n}$ as $t \rightarrow\left\{e^{i q k t / 2} a_{q}(t)\right\}_{q=1,2 \ldots, n}$. By virtue of (2-14), the function $t \rightarrow a_{2}(t)$ obeys

$$
\frac{d}{d t} a_{2}+2 \varepsilon g^{2 \overline{2}}=0 \text {. }
$$

Since $\varepsilon g^{2 \overline{2}}$ is real and nowhere zero, it follows that there are no solutions to (2-15) where $a_{2}(2 \pi)=a_{2}(0)$. This implies that there are no solutions to (2-15) with $\sigma_{2}(2 \pi)=\sigma_{2}(0)$. As a consequence, there are no solutions to (1-9) with domain $S^{1}=\mathbb{R} / 2 \pi \mathbb{Z}$.

A solution, $\mathfrak{c}: S^{1} \rightarrow \mathfrak{C}_{n}$, to (1-10) is deemed to be nondegenerate when (1-11) has trivial kernel as an operator on $C^{\infty}\left(S^{1} ; \mathfrak{c}^{*} T_{1,0} \mathfrak{C}_{n}\right)$. 
Lemma 2.4 The solutions to (1-10) that are described by Lemmas 2.1-2.3 are nondegenerate.

Proof of Lemma 2.4 In the case $n=1$, the identification of $\mathfrak{C}_{1}$ with $\mathbb{C}$ using the coordinate function $\sigma_{1}$ is a holomorphic isometry that makes (1-10) appear as the equation $\mathcal{L} z=0$ for $z$ a map from $S^{1}$ to $\mathbb{C}$ and with $\mathcal{L}$ as in (1-2). The linearized version of (1-10) appears as the operator $\mathcal{L}$. By assumption, this operator has trivial kernel. In the case when $\mu=0$, the functions $\left\{\sigma_{q}\right\}_{1 \leq q \leq n}$ identify $\mathfrak{C}_{n}$ with $\mathbb{C}^{n}$ and linearize (1-10). The linearized version of (1-10) about the symmetric vortex restricts to the $q$-th summand in $\mathbb{C}^{n}$ has nontrivial kernel if and only if such is the case for $(i / 2)(d / d t)+(1 / 2) q \mathrm{R} v$. The assumptions guarantee that this operator has trivial kernel.

\section{2.c Lifting maps from $S^{1}$ to $\mathfrak{C}_{n}$}

Focus for the moment on a given smooth map $\mathfrak{c}: S^{1} \rightarrow \mathfrak{C}_{n}$. Because $\mathfrak{C}_{n}$ is contractible, any such map lifts to a smooth map $\left.t \rightarrow(A, \alpha)\right|_{t}$ from $S^{1}$ into the space of pairs whose first component is a connection on the trivial bundle over $\mathbb{C}$ and whose second component is a section of this bundle. There are innumerable lifts; but any two differ by the action of a map from the circle into $C^{\infty}(\mathbb{C} ; U(1))$. To constrain the lifts under consideration, note that with $\mathfrak{c}$ given, there exists $R \geq 1$ such that $|\alpha| \geq \frac{1}{2}$ at all $(t, z) \in S^{1} \times \mathbb{C}$ with $|z| \geq R$. Consider in what follows lifts where $\alpha$ appears at points with $|z|$ sufficiently large as $|\alpha| z^{n} /|z|^{n}$.

Now suppose that a smooth map $A_{0}: S^{1} \rightarrow C^{\infty}(\mathbb{C} ; i \mathbb{R})$ has been given. Define a pair $(x, \iota)$ by writing

- $\frac{\partial}{\partial t} A-\bar{\partial} A_{0} d z-\bar{\partial} A_{0} d \bar{z}=2^{-1 / 2}(x d \bar{z}-\bar{x} d z)$.

- $\frac{\partial}{\partial t} \alpha+A_{0} \alpha=\iota$.

To put (2-17) in perspective, view $A_{0} d t+A$ as a connection, $A$, on the trivial complex line bundle over $S^{1} \times \mathbb{C}$. What is written in the first line is the contraction of $\partial / \partial t$ with the associated curvature 2 -form. What is written in the second line of (2-17) is the associated covariant derivative of $\alpha$ along the $S^{1}$ factor of $S^{1} \times \mathbb{C}$. As explained momentarily, there is a natural choice for $A_{0}$ that has the following property: Let $F_{A}$ denote $A$ 's curvature 2-form. Then the latter does not depend on the chosen lift of $\mathfrak{c}$. Meanwhile the covariant derivative $\nabla_{A} \alpha$ behaves in an equivariant fashion under a change of the lift.

To motivate this choice of $A_{0}$, note that the $L^{2}$-orthogonal projection at each $t \in S^{1}$ of $(x, \iota)$ into the vector space of $L^{2}$ solutions to (1-7) is insensitive to the choice 
for $A_{0}$. However, there is a unique choice of $A_{0}$ for which the pair $(x, \iota)$ actually defines an element in this vector space. This is the desired choice for $A_{0}$. To say more about this, note that the right hand equation in (1-7) is obeyed for any choice of $A_{0}$. It is also the case that the real part of the left hand equation is always obeyed. However, the imaginary part of the left hand equation in (1-7) demands that $A_{0}$ on each constant $t \in S^{1}$ copy of $\mathbb{C}$ obey

$$
\left(\frac{1}{4} d^{*} d\left(A_{0} \mid t\right)+\frac{1}{2}|\alpha|^{2} A_{0}\right)+\frac{1}{2} \operatorname{im}\left(\partial\left(\frac{\partial}{\partial t} a\right)+2^{-1 / 2} \bar{\alpha} \frac{\partial}{\partial t} \alpha\right)=0,
$$

where $a$ is obtained by writing $A=\frac{1}{2}(a d \bar{z}-\bar{a} d z)$. Given the large $|z|$ behavior of $\alpha$ as described in (2-1), it follows that this equation has a unique solution at each $t \in S^{1}$ that lies in $L_{1}^{2}(\mathbb{C} ; i \mathbb{R})$. This choice for $A_{0}$ guarantees that the pair $(x, \iota)$ is an $L^{2}$ solution to (1-7) at each $t \in S^{1}$.

As the solution to (2-18) varies smoothly with $t$, so does $(x, \iota)$ in $(2-17)$. What is said in Part 8 of Section 2.a implies that the lift of $\mathfrak{c}$ can be chosen so that the following is true: Let $\left\{z_{j}\right\}_{1 \leq j \leq n}$ denote the zeros of $\alpha$. Then

$$
\left|A_{0}\right|+\left|d\left(\left.A_{0}\right|_{t}\right)\right| \leq c_{\mathfrak{c}} \sum_{1 \leq j \leq n} e^{-\sqrt{2}\left|z-z_{j}\right|}
$$

at each $t \in S^{1}$. Here, $c_{\mathfrak{c}}$ is a constant that depends on the chosen lift.

The solution $A_{0}$ to (2-18) depends on the chosen lift of $\mathfrak{c}$ in the following way: Suppose that $t \rightarrow u(t)$ is a map from $S^{1}$ to $C^{\infty}(\mathbb{C} ; U(1))$. This map defines a new lift by changing $\left.(A, \alpha)\right|_{t}$ to $\left.\left(A^{\prime}, \alpha^{\prime}\right)\right|_{t}=\left.\left(A-u^{-1} d u, u \alpha\right)\right|_{t}$. Change $A_{0} \rightarrow A_{0}^{\prime}=$ $A_{0}-u^{-1}(\partial / \partial t) u$. Then $A_{0}^{\prime}$ obeys the $\left(A^{\prime}, \alpha^{\prime}\right)$ version of (2-18) and the resulting pair $\left(x^{\prime}, \iota^{\prime}\right)$ obeys the $\left(A^{\prime}, \alpha^{\prime}\right)$ version of (1-7).

\section{2.d $L_{1}^{2}$ maps from $S^{1}$ to $\mathfrak{C}_{n}$}

It is a straightforward business to define an $L_{1}^{2}$ map from $S^{1}$ to $\mathfrak{C}_{n}$ because the complex coordinates $\left\{\sigma_{q}\right\}_{1 \leq q \leq n}$ for $\mathfrak{C}_{n}$ can be used to identify $\mathfrak{C}_{n}$ with $\mathbb{C}^{n}$. With this identification understood, an $L_{1}^{2}$ map from $S^{1}$ to $\mathfrak{C}_{n}$ is simply an $L_{1}^{2}$ map, $t \rightarrow\left\{\sigma_{q}(t)\right\}_{1 \leq q \leq n}$ from $S^{1}$ to $\mathbb{C}^{n}$.

Because $L_{1}^{2}$ functions on $S^{1}$ are continuous, there is an equivalent way to define such a map: Fix $\varepsilon_{0}>0$ so that the map êxp as described in Part 8 of Section 2.a is defined on the radius $\varepsilon_{0}$ ball in $T_{1,0} \mathfrak{C}_{n}$. Let $\mathfrak{c}: S^{1} \rightarrow \mathfrak{C}_{n}$ denote a given smooth map. Fix an $L_{1}^{2}$ section, $\zeta$, of $\mathfrak{c}^{*} T_{1,0} \mathfrak{C}_{n}$ with pointwise norm less than $\varepsilon_{0}$. The map $t \rightarrow \mathfrak{c}_{* \zeta}(t)=\hat{\exp }_{\mathfrak{c}(t)} \zeta(t)$ defines an $L_{1}^{2}$ map from $S^{1}$ to $\mathfrak{C}_{n}$.

The remaining two parts of this subsection discuss two aspects of $L_{1}^{2}$ maps. 
Part 1 The second definition of an $L_{1}^{2}$ map gives a cheap way to define its lift as a map from $S^{1}$ into the space of solutions to (1-4). To explain, let $\mathfrak{c}: S^{1} \rightarrow \mathfrak{C}_{n}$ denote a given smooth map, and let $\zeta$ denote an $L_{1}^{2}$ section of $\mathfrak{c}^{*} T_{1,0} \mathfrak{C}_{n}$ with norm less than $\varepsilon_{0}$. Choose a lift of $\mathfrak{c}$ so as to give a pair, $(A, \alpha)$, this a smooth map from $S^{1}$ into the space of solutions to (1-4). Assume, as before that $\alpha$ is proportional to $z^{m}$ where $|z|$ is large. This lift of $\mathfrak{c}$ defines one for $\mathfrak{c}_{* \zeta}(t)$ : View $\zeta$ as an $L_{1}^{2}$ map from $S^{1}$ into $C^{\infty}(\mathbb{C} ; \mathbb{C} \oplus \mathbb{C})$ with $\left.\vartheta_{c} \zeta\right|_{t}=0$ at each $t \in S^{1}$, and then (2-9) defines the pair $\left(A^{\zeta}, \alpha^{\zeta}\right)$ and the desired lift of $\mathfrak{c}_{* \zeta}$ is the pair $\left(A_{0} d t+A^{\zeta}, \alpha^{\zeta}\right)$.

To say more, write $\zeta$ as a map $\left.t \rightarrow\left(q_{\zeta 0}, \varsigma_{\zeta 0}\right)\right|_{t}$ of $S^{1}$ into $C^{\infty}(\mathbb{C} ; \mathbb{C} \oplus \mathbb{C})$. Now let $f_{\zeta}$ denote the $L^{2}$ function on $S^{1}$ whose square is given by

$$
f_{\zeta}^{2}=\int_{\mathbb{C}}\left(\left|\frac{\partial}{\partial t} q_{\zeta 0}\right|^{2}+\left|\left(\frac{\partial}{\partial t}+A_{0}\right) \varsigma \zeta 0\right|^{2}\right)+\int_{\mathbb{C}}\left(|x|^{2}+|\iota|^{2}\right) \int_{\mathbb{C}}|\zeta|^{2} .
$$

Here, $A_{0}$ and $(x, \iota)$ are defined from $(A, \alpha)$ using (2-18) and (2-17). Note that a $\mathfrak{c}$-independent multiple of $f_{\zeta}$ bounds the $L^{2}$ norm of the covariant derivative of $\zeta$ 's incarnation as a section over $S^{1}$ of $\mathfrak{c}^{*} T_{1,0} \mathfrak{C}_{n}$. This covariant derivative is defined using the pullback by $\mathfrak{c}$ of the Levi-Civita connection on $T_{1,0} \mathfrak{C}_{n}$. Meanwhile, a $\mathfrak{c}$-independent multiple of the $L_{1}^{2}$ norm of the latter incarnation bounds $f_{\zeta}$.

With the preceding as background, define $\left(q_{\zeta}, \varsigma \zeta_{\zeta}\right)$ via (2-9) and (2-10); and set

$$
x_{\zeta}=x+\frac{\partial}{\partial t} q_{\zeta} \quad \text { and } \quad \iota \zeta=\iota+\left(\frac{\partial}{\partial t}+A_{0}\right) \varsigma \zeta,
$$

for the $\mathfrak{c}_{* \zeta}$ versions of the functions that are defined by (2-17). It follows from (2-2), (2-11) and (2-12) that there exists a constant, $\kappa$, that is independent of $\mathfrak{c}$ and of $\zeta$, with the following properties:

$$
\left|x_{\zeta}-x\right|+|\iota-\iota| \leq \kappa f_{\zeta}(t) \sum_{1 \leq j \leq n} e^{-\sqrt{2}\left|z-z_{j}(t)\right|} .
$$

Here, $t \rightarrow\left\{z_{j}(t)\right\}_{1 \leq j \leq n}$ is $\mathfrak{c}$ 's associated map from $S^{1}$ to $\operatorname{Sym}^{n}(\mathbb{C})$.

Part 2 What follows concerns two important points that play a prominent role in the next section. To set the stage, suppose that $\mathfrak{c}: S^{1} \rightarrow \mathfrak{C}_{n}$ is a smooth map and $\zeta$ is A section of $\mathfrak{c}^{*} T_{1,0} \mathfrak{C}_{n}$ with small $L_{1}^{2}$ norm. Lift $\mathfrak{c}$ as a pair, $(A, \alpha)$ so as to define $\left(A^{\zeta}, \alpha^{\zeta}\right)$. At each $t \in S^{1}$, the pair $\left(A^{\zeta}, \alpha^{\zeta}\right)$ defines the associated operator $\vartheta_{\mathfrak{c}_{* \zeta}(t)}$. Use $\Pi_{\mathfrak{c}_{* \zeta}(t)}$ to denote the $L^{2}(\mathbb{C} ; \mathbb{C} \oplus \mathbb{C})$-orthogonal projection onto the kernel of $\vartheta_{\mathfrak{c}_{* \zeta}(t)}$. As the lift of $\mathfrak{c}_{* \zeta}$ defines a continuous pair of connection and complex function, so the family $\left\{\Pi_{\mathfrak{c}_{* \zeta}(t)}\right\}_{t \in S^{1}}$ defines a continuous map from the circle into the space of bounded operators on $L^{2}(\mathbb{C} ; \mathbb{C} \oplus \mathbb{C})$. Use $\mathbb{K} \subset L^{2}\left(S^{1} \times \mathbb{C} ; \mathbb{C} \oplus \mathbb{C}\right)$ for the subspace of $\mathfrak{f} \in L^{2}\left(S^{1} \times \mathbb{C} ; \mathbb{C} \oplus \mathbb{C}\right)$ such that $\Pi_{\mathfrak{c}_{* \zeta}(t)} \mathfrak{f}=\mathfrak{f}$ for all $t \in S^{1}$. This is a closed, 
linear subspace. Introduce $\Pi_{\mathfrak{c}_{* \zeta}}$ to denote the $L^{2}$-orthogonal projection onto $\mathbb{K}$. Thus, $\left.\left(\Pi_{\mathfrak{c}_{* \zeta}} \mathfrak{f}\right)\right|_{t}=\Pi_{\mathfrak{c}_{* \zeta}(t)}\left(\left.\mathfrak{f}\right|_{t}\right)$.

The next lemma concerns this $\Pi_{\mathfrak{c}_{* \zeta}}$ The lemma introduces $\nabla_{t}$ to denote the covariant derivative on $C^{\infty}\left(S^{1} \times \mathbb{C} ; \mathbb{C} \oplus \mathbb{C}\right)$ that sends any given pair $\mathfrak{f}=(q, \varsigma)$ of functions on $S^{1} \times \mathbb{C}$ to the pair $\nabla_{t} \mathfrak{f}=\left((\partial / \partial t) q,\left(\partial / \partial t+A_{0}\right) \varsigma\right)$.

Lemma 2.5 The projection $\Pi_{\mathfrak{c}_{* \zeta}}$ maps $L_{1}^{2}\left(S^{1} \times \mathbb{C} ; \mathbb{C} \oplus \mathbb{C}\right)$ to itself. Moreover, there exists $\kappa \geq 1$ that depends on $\mathfrak{c}$ but not on $\zeta$ such that

$$
\left\|\left[\nabla_{t}, \Pi_{\mathfrak{c}_{* \zeta}}\right] \mathfrak{f}\right\|_{2}^{2} \leq \kappa\left(\left\|f_{\xi}\right\|_{2}^{2}+1\right)\left(\left\|\nabla_{t} \mathfrak{f}\right\|_{2}\|\mathfrak{f}\|_{2}+\|\mathfrak{f}\|_{2}^{2}\right),
$$

for all $\mathfrak{f} \in L_{1}^{2}\left(S^{1} \times \mathbb{C} ; \mathbb{C} \oplus \mathbb{C}\right)$.

Proof of Lemma 2.5 The commutator $\left[\nabla_{t}, \Pi_{\mathfrak{c}_{* \zeta}(\cdot)}\right]$ obeys

$$
\int_{\mathbb{C}}\left|\left[\nabla_{t}, \Pi_{\mathfrak{c}_{* \zeta}(\cdot)}\right] \mathfrak{f}\right|^{2} \leq c_{\mathfrak{c}}\left(f_{\zeta}^{2}+1\right) \int_{\mathbb{C}}|\mathfrak{f}|^{2},
$$

where $c_{\mathfrak{c}}$ is independent of $\lambda, \zeta$ and $t \in S^{1}$, but does depend on $\mathfrak{c}$. This last bound follows using (2-21) and (2-22) with the $k=0$ version of (2-2). Equation (2-23) implies the inequality that is asserted by Lemma 2.5. The latter implies that the projection $\Pi_{\mathfrak{c}_{* \zeta}}$ maps $L_{1}^{2}\left(S^{1} \times \mathbb{C} ; \mathbb{C} \oplus \mathbb{C}\right)$ to itself.

\section{2.e Surfaces and vortices}

Let $C$ denote a compact or noncompact complex curve and let $\pi: E \rightarrow C$ denote a complex, holomorphic line bundle equipped with a Hermitian metric and compatible connection. Use $S_{E} \subset E$ to denote the unit circle bundle in $E$. The integer $n$ version of the vortex bundle associated to $E$ is the fiber bundle $S_{E} \times_{S^{1}} \mathfrak{C}_{n}$. The latter is denoted by $\mathfrak{C}_{E, n}$; when needed, the projection to $C$ from $\mathfrak{C}_{E, n}$ is also denoted by $\pi$. This bundle $\mathfrak{C}_{E, n}$ is a holomorphic fiber bundle over $\mathbb{C}$. Let $V_{1,0} \mathfrak{C}_{E, n} \rightarrow \mathfrak{C}_{E, n}$ denote the $(1,0)$ component of the vertical tangent bundle with respect to the projection to $C$. Thus $V_{1,0}=S_{E} \times S^{1} T_{1,0} \mathfrak{C}_{n}$.

There exists in this context a version of the $d$-bar operator that takes a section, $\mathfrak{c}$, of $\mathfrak{C}_{E, n}$ to a section, $\bar{\partial} \mathfrak{c}$, of the bundle $\mathfrak{c}^{*} V_{1,0} \otimes T^{0,1} C \rightarrow C$. It is defined as follows: With $\mathfrak{c}$ viewed as an $S^{1}$-invariant map from $S_{E}$ to $\mathfrak{C}_{n}$, its differential defines a linear map from $T S_{E}$ to $\mathfrak{c}^{*} T_{1,0} \mathfrak{C}_{n}$. Restriction of this differential to the horizontal subbundle in $T S_{E}$ as defined by the given Hermitian connection gives the desired section of $\mathfrak{c}^{*} V_{1,0} \mathfrak{C}_{E, n} \otimes T^{0,1} C$. 
Let $v_{C}$ denote a section of $T^{0,1} C$ and $\mu_{C}$ a section of $E^{2} \otimes T^{0,1} C$. With $v=$ $v_{C}$ and $\mu=\mu_{C}$, what is written in (1-9) defines a section, $f\left(h\right.$, over $\mathfrak{C}_{E, n}$ of the bundle $\pi^{*} T^{0,1} C$. This understood, use $\nabla^{1,0} h$ to denote the corresponding section of $V_{1,0} \mathfrak{C}_{E, n} \otimes \pi^{*} T^{0,1} C$. Of interest here are sections of $\mathfrak{C}_{E, n}$ that obey the equation

$$
\bar{\partial} \mathfrak{c}+\mathfrak{c}^{*} \nabla^{1,0} \mathfrak{h}=0 .
$$

To see something of what this looks like, note that in the case $n=1$, the identification $\mathfrak{C}_{1}=C$ given by the function $\sigma_{1}$ on $\mathfrak{C}_{1}$ identifies the expression on the right hand side of $(2-24)$ with the $\mathbb{R}$-linear operator from $C^{\infty}(C ; E)$ to $C^{\infty}\left(C ; E \otimes T^{1,0} C\right)$ that sends any given section $\zeta$ to

$$
\bar{\partial} \zeta+v_{C} \zeta+\mu_{C} \bar{\zeta}
$$

When $\mu_{C}=0$, the identification $\mathfrak{C}_{n}=\mathbb{C}^{n}$ identifies the right hand side of (2-24) as the $\mathbb{C}$-linear operator from $C^{\infty}\left(C ; \bigoplus_{1 \leq q \leq n} E^{q}\right)$ to $C^{\infty}\left(C ;\left(\bigoplus_{1 \leq q \leq n} E^{q}\right) \otimes T^{0,1} C\right)$ that acts in a diagonal fashion as

$$
\bar{\partial}+q v_{C}
$$

on the $q$-th summand. These are the two cases of paramount interest in what follows.

\section{2.f Banach spaces of sections of $\mathfrak{C}_{E, n}$}

The bundle $V_{1,0} \mathfrak{C}_{E, n} \rightarrow \mathfrak{C}_{E, n}$ is a rank $n$, complex vector bundle. As such, it inherits a Hermitian structure from the $L^{2}$ metric on $\mathfrak{C}_{n}$ and a fiberwise covariant derivative. The latter with the given Hermitian connection on $S_{E}$ defines a connection on this vector bundle. Fix a smooth section, $\mathfrak{c}$, of $\mathfrak{C}_{E, n}$. The pullback by $\mathfrak{c}$ of this connection on $V_{1,0} \mathfrak{C}_{E, n} \rightarrow \mathfrak{C}_{E, n}$ gives the bundle $\mathfrak{c}^{*} V_{1,0} \mathfrak{C}_{E, n}$ a covariant derivative. The latter can be used to define Sobolev spaces of sections. Of interest here are $L_{1}^{2}$ sections with apriori pointwise bounds. What follows describes a convenient Banach space of such sections. The space defined below is a version of a space used by Morrey in [3].

The definition of this Banach space requires the choice of a positive constant, $v$, but with $v<1 / 100$. Three norms on the space of smooth, compactly supported sections over $C$ of $\mathfrak{c}^{*} V_{1,0} \mathfrak{C}_{E, n}$ are defined in the next equation. The first is the standard $L_{1}^{2}$ norm. Then second and third require the constant $v$. These norms are defined by declaring the square of their respective values on any given section $\zeta$ are

- $\|\zeta\|_{\mathcal{K}_{1}^{2}}^{2}=\int_{\mathbb{C}}\left(|\nabla \zeta|^{2}+|\zeta|^{2}\right)$.

- $\|\zeta\|_{\mathcal{K} *}^{2}=\sup _{p \in C, \rho<1} \rho^{-v} \int_{\operatorname{dist}(\cdot, p)<p}|\nabla \zeta|^{2}$.

- $\|\zeta\|_{\mathcal{K}}^{2}=\|\zeta\|_{\mathcal{K}_{1}^{2}}^{2}+\|\zeta\|_{\mathcal{K} *}^{2}$. 
Here, $\nabla$ denotes the aforementioned covariant derivative on $C^{\infty}\left(C ; \mathfrak{c}^{*} V_{1,0} \mathfrak{C}_{E, n}\right)$. Meanwhile, the function $\operatorname{dist}(\cdot, p)$ denotes the metric distance on $C$ to the point $p$.

Use $\mathcal{K}_{\mathfrak{c} *}$ and $\mathcal{K}_{\mathfrak{c}}$ to denote the respective completions of the space of compactly supported, smooth sections of $\mathfrak{c}^{*} V_{1,0} \mathfrak{C}_{E, n}$ with respect to the norms $\|\cdot\|_{\mathcal{K} *}$ and $\|\cdot\|_{\mathcal{K}}$. By definition, $\mathcal{K}_{\mathfrak{c}}$ is a subvector space of $\mathcal{K}_{\mathfrak{c} *}$.

The norm $\|\cdot\|_{\mathcal{K} *}$ is somewhat stronger than the $L_{1 ; \text { loc }}^{2}$ norm. Indeed, as stated in the next lemma, elements of $\mathcal{K}_{\mathfrak{c} *}$ are bounded and Hölder continuous. Moreover, if $M$ is a compact 3-manifold with a given contact 1 -form as in Section 1 , and if $C$ is a pseudoholomorphic subvariety in $\mathbb{R} \times M$ of the sort described in Section 1.e, then sections of $\mathcal{K}_{\mathfrak{c} *}$ converge uniformly to zero as $|s| \rightarrow \infty$ on $C$.

Lemma 2.6 With $v$ and $C$ as just described, there is a constant $\kappa \geq 1$ such that if $\mathfrak{c} \in C^{\infty}\left(C ; \mathfrak{C}_{E, n}\right)$ and $\zeta \in \mathcal{K}_{\mathfrak{c} *}$ then $\sup _{C}|\zeta| \leq \kappa\|\zeta\|_{\mathcal{K}_{*}}$. In fact, each element in $\mathcal{K}_{\mathfrak{c} *}$ (and hence $\mathcal{K}_{\mathfrak{c}}$ ) is Hölder continuous with exponent $\kappa^{-1}$, and the inclusion of $\mathcal{K}_{\mathfrak{c} *}$ into the Banach space of such Hölder continuous sections defines a continuous map. If $C$ is a pseudoholomorphic subvariety of the sort described in Section 1.e, then $\mathcal{K}_{\mathfrak{c} *}$ includes into the Banach space of Hölder continuous section with exponent $\kappa^{-1}$ that have limit zero as $|s| \rightarrow \infty$; and the latter inclusion is a bounded, linear map. However, the norm of this map depends on $\mathfrak{c}$.

Proof of Lemma 2.6 See Morrey [3, Theorem 3.5.2] for the proof that elements in $\mathcal{K}_{\mathfrak{c} *}$ are Hölder continuous. In the case when $C$ is of the sort described in Section 1.e, the uniform decay to zero as $|s| \rightarrow \infty$ follows from this fact as it implies that $\mathcal{K}_{\mathfrak{c} *}$ sits in the $C^{0}$ closure of the space of compactly supported sections of $\mathfrak{c}^{*} V_{1,0} \mathfrak{C}_{E, n}$.

The Banach space $\mathcal{K}_{\mathfrak{c} *}$ is introduced because it is relatively easy to work with, and because its small normed elements can be used to define deformations of the given section $\mathfrak{c}$. In particular, given Lemma 2.6 and given what is said in Part 8 of Section 2.a about the map êxp, there exists $\varepsilon_{0}>0$ with the following significance:

Fix $\mathfrak{c} \in C^{\infty}\left(C ; \mathfrak{C}_{E, n}\right)$. If $\zeta \in \mathcal{K}_{\mathfrak{c} *}$ obeys $\|\zeta\|_{\mathcal{K}}<\varepsilon_{0}$ then $\mathfrak{c}_{* \zeta}=\operatorname{expp}_{\mathfrak{c}}(\zeta)$ defines a Hölder continuous section of $\mathfrak{C}_{E, n}$.

An $L^{2}$ version of the Banach space $\mathcal{K}_{\mathfrak{c}}$ is needed for sections of $\mathfrak{c}^{*} V_{1,0} \mathfrak{C}_{E, n} \otimes T^{0,1} C$. This $L^{2}$ version is denoted by $\mathcal{L}_{\mathfrak{c}}$; it is the completion of the space of compactly supported sections of $C^{\infty}\left(C ; \mathfrak{c}^{*} V_{1,0} \mathfrak{C}_{E, n} \otimes T^{0,1} C\right)$ using the norm whose square sends a section $\zeta$ to

$$
\int_{C}|\zeta|^{2}+\sup _{p \in C, \rho<1} \rho^{-v} \int_{\operatorname{dist}(\cdot, p)<\rho}|\zeta|^{2}
$$


The Banach space $\mathcal{L}_{\mathfrak{c}}$ is used when considering the linearized version of (2-24). Thi linearization is defined at a section $c \in C^{\infty}\left(C ; \mathfrak{C}_{E, n}\right)$ as a linear operator that takes any given section of $\mathfrak{c}^{*} V_{1,0} \mathfrak{C}_{E, n}$ and gives back a section of $\mathfrak{c}^{*} V_{1,0} \mathfrak{C}_{E, n} \otimes T^{0,1} C$. This operator can be written schematically as

$$
\zeta \rightarrow \bar{\partial}_{\mathfrak{c}} \zeta+\left.\left(\nabla_{\zeta_{\mathbb{R}}} \nabla^{1,0} \hbar\right)\right|_{\mathfrak{c}}
$$

where $\bar{\partial}_{\mathfrak{c}}$ here denotes the $d$-bar operator on the space of sections of $\mathfrak{c}^{*} V_{1,0} \mathfrak{C}_{E, n}$ that is defined using the pullback via $\mathfrak{c}$ of the Levi-Civita connection. Meanwhile, $\zeta_{\mathbb{R}}$ denotes the section of the vertical tangent bundle of $\mathfrak{c}$ 's pullback of the (real) vertical tangent bundle to $\mathfrak{C}_{E, n}$ that is defined by $\zeta$.

Lemma 2.7 The operator that is depicted in (2-30) defines a bounded operator $L_{1}^{2}\left(C ; \mathfrak{c}^{*} V_{1,0} \mathfrak{C}_{n}\right)$ to $L^{2}\left(C ; \mathfrak{c}^{*} V_{1,0} \mathfrak{C}_{n} \otimes T^{0,1} C\right)$. Moreover, there exists $\kappa \geq 1$ such that if $D \subset C$ is a ball of radius $\kappa^{-1}$ and if $\zeta$ has compact support in $D$, then

$$
\left\|\bar{\partial}_{\mathfrak{c}} \zeta+\left.\left(\nabla_{\zeta_{\mathbb{R}}} \nabla^{1,0} h\right)\right|_{\mathfrak{c}}\right\|_{\mathcal{L}} \geq c_{0}^{-1}-\|\zeta\|_{\mathcal{K}}
$$

As a consequence, if the operator $\zeta \rightarrow \bar{\partial}_{\mathfrak{c}} \zeta+\left.\left(\nabla_{\zeta_{\mathbb{R}}} \nabla^{1,0} h\right)\right|_{c}$ is Fredholm from $L_{1}^{2}$ to $L^{2}$, then it is Fredholm with the same index, kernel and cokernel as a map from $\mathcal{K}_{\mathfrak{c}}$ to $\mathfrak{L}_{\mathfrak{c}}$. In particular, if it is Fredholm from $L_{1}^{2}$ to $L^{2}$ and if its $L^{2}$ cokernel is trivial, then this operator has a bounded inverse mapping $\mathcal{K}_{\mathfrak{c}}$ to the $L^{2}$-orthogonal complement in $\mathfrak{L}_{\mathfrak{c}}$ of the kernel of its $L^{2}$ adjoint.

Proof of Lemma 2.7 The claims follow from [3, Theorems 3.5.2 and 5.4.1].

The Banach spaces $\mathcal{K}_{\mathfrak{c}}$ and $\mathcal{K}_{\mathfrak{c} *}$ are used later in this article with the two special cases that are described at the end of the preceding subsection. Recall that the first case is that with $n=1$. In this case, the isometry between $\mathfrak{C}_{1}$ and $\mathbb{C}$ that is supplied by the $k=1$ version of (1-5) identifies $C^{\infty}\left(C, \mathfrak{C}_{E, 1}\right)$ with $C^{\infty}(C ; E)$. With this identification understood, the Banach spaces $\mathcal{K}_{\mathfrak{c} *}$ and $\mathcal{K}_{\mathfrak{c}}$ are the respective completions of the space of compactly supported sections of $C^{\infty}(C ; E)$ using the norm whose square is given by the relevant version of (2-27) where $\zeta$ is a section of $E$ and $\nabla$ denotes the covariant derivative from the connection $A_{E}$. With $C^{\infty}\left(C ; \mathfrak{C}_{E, 1}\right)$ viewed as $C^{\infty}(C ; E)$, the operator in (2-30) is that in (2-25).

The second case is that where $\mathbb{C}=\mathbb{R} \times S^{1}$, the bundle $E$ is given as the trivial bundle $\left(\mathbb{R} \times S^{1}\right) \times \mathbb{C}$ and the function $\mu$ that appears in the equation for $h$ is taken to equal to zero. As remarked previously, (2-24) in this case is also linear, now a direct sum of $n$ operators, these acting on $\bigoplus_{1 \leq q \leq n} C^{\infty}\left(\mathbb{R} \times S^{1} ; \mathbb{C}\right)$ where the operator on the $q$-th summand is $\bar{\partial}+q v$. The norm for the Banach spaces $\mathcal{K}_{\mathfrak{c} *}$ and $\mathcal{K}_{\mathfrak{c}}$ now depend on the choice for $\mathfrak{c}$. 


\section{2.g Lifts of sections of $\mathfrak{C}_{E, n}$}

Let $\pi: E \rightarrow C$ denote the projection map. What follows describes how to lift a section of $\mathfrak{C}_{E, n}$ so as to get a pair whose first component is a connection on $\pi^{*} E^{n}$ and whose second component is a section of this same bundle. It then goes on to discuss various properties of such lifts. The discussion has three parts.

Part 1 To set the stage, remark that $\pi^{*} E \rightarrow E$ has a canonical section; the latter, $\mathfrak{s}$, assigns to any given point $\eta \in E$ the point $(\eta, \eta) \in E \times_{C} E$. This bundle also has a canonical connection; this is the pullback, $\theta$, of the given Hermitian connection on $E$. This understood, the $\left(\pi^{*} E\right)^{*}$ valued $1-$ form $\nabla_{\theta} \overline{\mathfrak{s}}$ pulls back to each fiber of $E$ so as to span the $(0,1)$ cotangent bundle of the fiber. This 1 -form annihilates the $\theta$-horizontal subspace in $T E$.

With these preliminaries in hand, suppose that $\mathfrak{c}$ is a given smooth section of $\mathfrak{C}_{E, n}$. Then $\mathfrak{c}$ can be lifted as a pair $(\mathbb{A}, \alpha)$ where $\mathbb{A}$ a smooth, Hermitian connection on the line bundle $\pi^{*} E^{n} \rightarrow E$ and $\alpha$ is a section of this bundle. The connection $\mathbb{A}$ here is taken equal to

$$
\mathbb{A}=n \theta+A_{0}+A
$$

where the notation is as follows: First, $A$ is an $i$-valued 1 -form on $E$ that annihilates the horizontal vectors in $T E$. Thus, $A$ can be written as $\frac{1}{2}\left(a \nabla_{\theta}, \overline{\mathfrak{s}}-\bar{a} \nabla_{\theta}, \mathfrak{s}\right)$ where $a$ is a section of $\pi^{*} E$. In addition $(A, \alpha)$ solves (1-4) along each fiber of $E$; and in doing so it defines the equivalence class of $\mathfrak{c}$. Finally, $A_{0}$ is a section over $E$ of $\pi^{*}\left(i T^{*} C\right)$ that is given along each fiber as the unique, $L^{2}$ solution to the equation

$$
\left(\frac{1}{4} d^{V \dagger} d^{V}\left(A_{0}\right)+\frac{1}{2}|\alpha|^{2} A_{0}\right)+\frac{1}{2} \operatorname{im}\left(\partial^{V}\left(\nabla_{\theta}^{H} a\right)+2^{-1 / 2} \bar{\alpha} \nabla_{\theta}^{H} \alpha\right)=0 .
$$

Here, $d^{V}$ and $\partial^{V}$ are the respective exterior derivative and holomorphic derivative along the fibers of $\pi$ and $\nabla_{\theta}^{H}$ denotes the horizontal part of the covariant derivative that is defined by $\theta$. By way of an example, $\nabla_{\theta}^{H} \mathfrak{s}=0$.

With $A_{0}$ understood, introduce the pair $(x, \iota)$ by

$$
x=2^{-1 / 2}\left(\nabla_{\theta}^{H} a-2 \bar{\partial}^{V} A_{0}\right) \quad \text { and } \quad \iota=\left(\nabla_{\theta}^{H}+A_{0}\right) \alpha .
$$


Then $(x, \iota)$ restrict to each fiber of $\pi: E \rightarrow C$ as a solution to (1-7). Note that the lift of $(A, \alpha)$ can be chosen so that they, the pair $(x, \iota)$ and $A_{0}$ obey

- $A=(1 / 2) n\left(\overline{\mathfrak{s}}^{-1} \nabla_{\theta} \overline{\mathfrak{s}}-s^{-1} \nabla_{\theta} \mathfrak{s}\right)+v$ and $\alpha=\mathfrak{s}^{n} /|\mathfrak{s}|^{n}+r$ where $|v|+|r| \leq$ $c_{\mathfrak{c}} e^{-\sqrt{2}|\mathfrak{s}|}$ at points where $|\mathfrak{s}| \gg 1$.

- $|x|+|\iota| \leq c_{\mathfrak{c}} e^{-\sqrt{2}|\mathfrak{s}|}$.

- $\left|A_{0}\right| \leq c_{\mathfrak{c}}^{\prime} e^{-\sqrt{2}|\mathfrak{s}|}$.

Here, $c_{\mathfrak{c}}$ is a constant that depends on the section $\mathfrak{c}$. Meanwhile, $c_{\mathfrak{c}}^{\prime}$ depends on the chosen lift of $\mathfrak{c}$. A lift of the sort just described is used implicitly below.

A change in the connection $\theta$ to $\theta+\Gamma$ changes $(x, \iota)$ to

$$
(x, \iota) \rightarrow(x, \iota)-\Gamma\left(2^{-1 / 2}\left(1-|\alpha|^{2}\right), \partial_{A}^{V} \alpha\right) .
$$

Thus, the change adds to $(x, \iota)$ a multiple of the solution to (1-7) along each fiber that is given by (2-3).

Part 2 As is explained next, the lift of $\mathfrak{c}$ as $(\mathbb{A}, \alpha)$ gives a canonical way to lift $\mathfrak{c}_{* \zeta}$ when $\zeta$ is any given small normed element in $\mathcal{K}_{\mathfrak{c} *}$. To begin the story, remark that the chosen lift of $\mathfrak{c}$ defines the rank $n$, complex vector bundle $\mathcal{K}_{\mathfrak{c}} \rightarrow C$ whose fiber at any given point $p \in C$ is the kernel of the operator $\vartheta_{\mathfrak{c}}$ along $\left.E\right|_{p}$. Here, $\vartheta_{\mathfrak{c}}$ is defined in (2-6) using $(\mathbb{A}, \alpha)$ along $\left.E\right|_{p}$. The bundle $\mathcal{K}_{\mathfrak{c}}$ is canonically isomorphic to $\mathfrak{c}^{*} V_{1,0} \mathfrak{C}_{E, n}$ and so any given section of the latter can be viewed as a section of $\mathcal{K}_{\mathfrak{c}}$. This understood, a corresponding fiberwise version of (2-9) is used to define the lift of $\mathfrak{c}_{* \zeta}$ for any given section $\zeta$. Indeed, define $\left(q_{\zeta}, \zeta \zeta\right)$ fiberwise by (2-9). Thus, $q \zeta$ is a section of $\pi^{*} E$ and $\zeta \zeta$ one of $\pi^{*} E^{n}$. Granted this notation, the lift of $\mathfrak{c}_{* \zeta}$ is given by

$$
\left(n \theta+A_{0}+A+2^{-1 / 2}\left(q_{\zeta} \nabla_{\theta} \overline{\mathfrak{s}}-\bar{q}_{\zeta} \nabla_{\theta} \mathfrak{s}\right), \alpha+\varsigma \zeta\right) .
$$

In what follows $\left(A^{\zeta}, \alpha^{\zeta}\right)$ is used to denote $\left(A+2^{-1 / 2}\left(q_{\zeta} \nabla_{\theta} \overline{\mathfrak{s}}-\bar{q}_{\zeta} \nabla_{\theta} \mathfrak{s}\right), \alpha+\varsigma_{\zeta}\right)$.

There are analogs for $\mathfrak{c}_{* \zeta}$ of (2-21) and (2-22). These are defined as follows: Introduce the pair $\left(x_{\zeta}, \iota_{\zeta}\right)$ by

$$
x_{\zeta}=x+\nabla_{\theta} q_{\zeta} \quad \text { and } \quad \iota \zeta=\iota+\left(\nabla_{\theta}+A_{0}\right) \varsigma \zeta .
$$

Then

$$
\left|x_{\zeta}-x\right|+|\iota \zeta-\iota| \leq \kappa f_{\zeta} \sum_{1 \leq j \leq n} e^{-\sqrt{2}\left|\mathfrak{s}-\mathfrak{s}_{j}\right|},
$$

where the notation is as follows: First, $\kappa$ is a constant that is independent of $\mathfrak{c}$. Second, $\left\{\mathfrak{s}_{j}\right\} \in \operatorname{Sym}^{n}(E)$ is the zero locus of $\alpha$. Finally, $f_{\xi}$ is the function on $C$ whose square 
at a given $p \in \Sigma$ is

$$
f_{\zeta}^{2}(p)=\int_{\left.E\right|_{p}}\left(\left|\nabla_{\theta} q_{\zeta 0}\right|^{2}+\left|\left(\nabla_{\theta}+A_{0}\right) \zeta \zeta 0\right|^{2}\right)+\int_{\left.E\right|_{p}}\left(|x|^{2}+|\iota|^{2}\right) \int_{\left.E\right|_{p}}|\zeta|^{2}
$$

Here, $\left(q_{\zeta 0}, \varsigma_{\zeta 0}\right)$ are the components of $\zeta$. Note that

$$
\text { - } c_{0}^{-1}\|\zeta\|_{\mathcal{K}_{1}^{2}}^{2} \leq \int_{C}|\zeta|^{2}+\int_{C} f_{\zeta}^{2} \leq c_{0}\|\zeta\|_{\mathcal{K}_{1}^{2}}^{2} .
$$

$$
\text { - } c_{0}^{-1}\|\zeta\|_{\mathcal{K} *}^{2} \leq \sup _{p \in C, \rho<1} \rho^{-v}\left(\int_{\operatorname{dist}(\cdot, p)<\rho}|\zeta|^{2}+\int_{\operatorname{dist}(\cdot, p)<\rho} f_{\zeta}^{2}\right)
$$

Here $c_{0} \geq 1$ is independent of $c$ and $\zeta$. Note with regards to the first item that the $L^{2}$ norm of $\zeta$ as a section over $C$ of $\mathfrak{c}^{*} V_{1,0} \mathfrak{C}_{E, n}$, is the same, up to a fixed multiplicative constant, as the $L^{2}$ norm over $E$ of $\zeta$ as a section over $E$ of $\pi^{*} E \oplus \pi^{*} E^{n}$.

Part 3 The final item on the agenda for this section concerns the analog of Lemma 2.5 and (2-23) for sections of $\mathfrak{C}_{E, n}$. To set the stage, let $\mathfrak{c}$ denote a given smooth section of $\mathfrak{C}_{E, n}$. Introduce $\nabla$ to denote the covariant derivative on $C^{\infty}\left(E ; \pi^{*} E \oplus\left(\pi^{*} E\right)^{n}\right)$ that acts as $\nabla_{\theta}+d^{E}$ on the first summand and $\nabla_{\mathbb{A}}$ on the second. Here, $\mathbb{A}$ is defined by (2-31) with $A_{0}$ defined by (2-32). Use this covariant derivative to define the Sobolev space $L_{1}^{2}\left(E ; \pi^{*} E \oplus \pi^{*} E^{n}\right)$. To be precise, the latter is the completion of the space of compactly supported sections over $E$ of $\pi^{*} E \oplus \pi^{*} E^{n}$ with the norm whose square sends a section $\mathfrak{f}$ to

$$
\int_{E}\left(|\nabla \mathfrak{f}|^{2}+|\mathfrak{f}|^{2}\right)
$$

To finish with these preliminaries, fix a small normed section, $\zeta \in \mathcal{K}_{\mathfrak{c} *}$ so as to define the section $\mathfrak{c}_{* \zeta}$ of $\mathfrak{C}_{E, n}$. Use the associated pair $\left(A^{\zeta}, \alpha^{\zeta}\right)$ to define on each fiber the operator $\vartheta_{\mathfrak{c}_{* \zeta}(\cdot)}$ as in (2-6). Let $\Pi_{\mathfrak{c}_{* \zeta}(\cdot)}$ denote the fiberwise $L^{2}$ orthogonal projection to the kernel of this operator. Apriori, the latter defines a continuous section over $C$ of a vector bundle that is associated to $S_{E}$ and whose fiber is the vector space of bounded, finite rank operators on $L^{2}(\mathbb{C} ; \mathbb{C} \oplus \mathbb{C})$. Let $\mathbb{K} \subset L^{2}\left(E ; \pi^{*} E \oplus \pi^{*} E^{n}\right)$ denote the

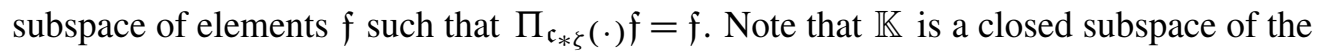
Hilbert space $L^{2}\left(E ; \pi^{*} E \oplus \pi^{*} E^{n}\right)$. Use $\Pi_{\mathfrak{c}_{* \zeta}}$ to denote the $L^{2}$ orthogonal projection onto $\mathbb{K}$.

With the stage now set, the following lemma plays a key role in the construction of the map $\Psi^{r}$. 
Lemma 2.8 The projection $\Pi_{\mathfrak{c}_{* \zeta}}$ maps $L_{1}^{2}\left(E ; \pi^{*} E \oplus \pi^{*} E^{n}\right)$ to itself as a bounded operator. Moreover,

$$
\|\left[\nabla, \Pi_{\left.\mathfrak{c}_{* \zeta}(\cdot)\right]} \mathfrak{f}_{2}^{2} \leq \kappa\|\zeta\|_{\mathcal{K} *}^{2}\left(\|\nabla \mathfrak{f}\|_{2}+\|\mathfrak{f}\|_{2}\right)^{2}+\kappa\|\mathfrak{f}\|_{2}^{2} .\right.
$$

Here, $\kappa>1$ depends only on the section $\mathfrak{c}$.

This lemma is proved momentarily.

The following lemma is used in the proof Lemma 2.8 and elsewhere.

Lemma 2.9 Let $\theta:[0, \infty) \rightarrow \mathbb{R}$ denote a smooth function such that the function $z \rightarrow \theta(|z|)$ on $C$ is square integrable. Use $\theta_{E}: E \rightarrow \mathbb{R}$ to denote the function $\theta(|s|)$. Let $\mathfrak{f}$ denote an element of $L_{1}^{2}\left(E ; \pi^{*} E \oplus \pi^{*} E^{n}\right)$. Then function on $C$ given by

$$
\int_{\pi^{-1}(\cdot)} \theta_{E}|\mathfrak{f}|
$$

is an $L_{1}^{2}$ function with $L_{1}^{2}$ norm bounded by a $\theta$-dependent multiple of $\|\nabla \mathfrak{f}\|_{2}+\|\mathfrak{f}\|_{2}$.

Proof of Lemma 2.9 This follows using Hölder's inequality given that the exterior derivative of the indicated function on $C$ is bounded by a multiple of $\int_{\pi^{1}(\cdot)} \theta_{E}|\nabla \mathfrak{f}|$.

Proof of Lemma 2.8 The assertion that $\Pi_{\mathfrak{c}_{* \zeta}}$ maps $L_{1}^{2}\left(E ; \pi^{*} E \oplus \pi^{*} E^{n}\right)$ to itself follows from the inequality. To prove the inequality, note first that there is an analog here of (2-23). This analog replaces the integration domain $C$ by any given fiber of $\pi$ and it replaces $\nabla_{t}$ with $\nabla$. This version of (2-23) follows using (2-2), (2-11), (2-12) and (2-38). Granted this $\nabla_{t} \rightarrow \nabla$ and $\left.C \rightarrow E\right|_{(\cdot)}$ version of (2-23) use [3, Lemma 5.4.1] with Lemma 2.9 to obtain the desired inequality.

\section{The proof of Theorem 1.1}

The purpose of this section is to first construct the map $\Phi^{r}$ and then prove the assertions made in Theorem 1.1. To this end, fix a once and for all a coclosed $1-$ form $\mu \in \Omega$ with $\mathcal{P}$ norm less than 1 .

To start the construction, fix a finite set, $\Theta$, whose elements are pairs $(\gamma, m)$ where $\gamma$ is a Reeb orbit and $m$ is a positive integer. Require that distinct elements have distinct Reeb orbits. Assign to each $(\gamma, m) \in \Theta$ a map $\mathfrak{c}_{\gamma}: S^{1} \rightarrow \mathfrak{C}_{m}$ that solves (1-10) and is nondegenerate in the sense that the associated version of (1-11) has trivial kernel. This data constitutes an element, $\mathfrak{J}_{0}$, in the set $\mathfrak{C}^{*}{ }^{*}$. The first task here is to construct from $\mathfrak{J}_{0}$ a solution to the large $r$ versions of (1-13). 
The first and simplest case has $\Theta=\varnothing$. The corresponding solution in this case is described by [7, Proposition 2.8]. This proposition is restated below. The proposition refers to the function $\mathrm{E}$ given by (1-12).

Proposition 3.1 Suppose that the $\operatorname{Spin}_{\mathbb{C}}$ structure is such that $\mathbb{S}=I_{\mathbb{C}} \oplus K^{-1}$. There exists $r_{I}>1$ and $\delta \in\left(0, \frac{1}{2}\right)$ with the following significance: Fix $\mu \in \Omega$ with $\mathcal{P}$ norm less than 1 and fix $r \geq r_{I}$. Then there exists a unique gauge equivalence class of solution to (1-13) with the norm of the spinor component nowhere less than $1-\delta$. Moreover, E has an $r$ independent upper bound on this equivalence class.

Assume henceforth that $\Theta$ is nonempty. The spinor bundle $\mathbb{S}$ for the relevant $\operatorname{Spin}_{\mathbb{C}}$ structure decomposes as $E \oplus E K^{-1}$ where the first Chern class of $E$ is Poincare' dual to the class in $H_{1}(M ; \mathbb{Z})$ that is given by the formal sum $\sum_{(\gamma, m) \in \Theta} m \gamma$. This

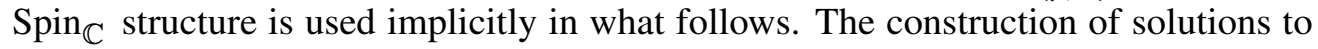
(1-13) from $\mathfrak{J}_{0}$ uses much the same technology that is used in [7] to prove the latter's Proposition 2.8. The salient new input involves the solutions to (1-10). As outlined in [8, Section 5b], the first part of the construction uses the solutions to (1-10) to construct a pair in $\operatorname{Conn}(E) \times C^{\infty}(M ; \mathbb{S})$ that almost solves (1-10). The perturbation-theoretic approach used for [7, Proposition 2.8] augmented with techniques from the article $\mathrm{Gr}=\mathrm{SW}$ in [5] are then brought to bear to prove that there is a unique solution to (1-10) that is near to this pair in $\operatorname{Conn}(E) \times C^{\infty}(M ; \mathbb{S})$. Sections 3.a-3.g supply the existence proof. The uniqueness assertion is proved in Section 2 of the fourth paper in this series [10]. Section 3.g proves the assertions made by Theorem 1.1.

\section{3.a Configurations that nearly solve (1-13)}

Let $\varepsilon_{0}>0$ be as described in the Part 8 of Section 2.a's discussion of exponential maps. The purpose of this subsection is to associate a Hermitian connection on $E$ with $L^{2}$ curvature and an $L_{1}^{2}$ section of $\mathbb{S}$ to the following data:

- A set finite set $\Theta$ whose typical element is a pair $(\gamma, m)$, where $\gamma$ is a Reeb orbit and $m$ is a positive integer. There is no need to assume that $\gamma$ is nondegenerate. These are constrained as follows:

(i) No two pairs share the same Reeb orbit.

(ii) $\sum_{(\gamma, m) \in \Theta} m \gamma$ is Poincaré dual to $c_{1}(E)$.

- A set $\mathfrak{J}=\left\{\left(\mathfrak{c}_{\gamma}, \zeta_{\gamma}\right)\right\}_{(\gamma, m) \in \Theta}$ where

(i) $\mathfrak{c}_{\gamma}$ is a smooth map from $S^{1}$ into $\mathfrak{C}_{m}$.

(ii) $\zeta_{\gamma}$ is an $L_{1}^{2}$ section over $S^{1}$ of $\mathfrak{c}_{\gamma}^{*} T_{1,0} \mathfrak{C}_{m}$ with pointwise norm less than $\varepsilon_{0}$.

The pair of connection and spinor with the label $\Theta$ and $\mathfrak{J}$ is denoted below by $\mathfrak{c}_{\Theta \mathfrak{J}}$. 
Step 1 As described in Section 1.a, each Reeb orbit $\gamma$ from $\Theta$ has an associated tubular neighborhood coordinate chart, $\varphi_{\gamma}$, that maps $S^{1} \times D$ into $M$ with $D \subset \mathbb{C}$ a small radius disk about the origin. Such a chart can and should be chosen so that the following is true: There is an orthonormal basis for $T^{*} M$ near $\gamma$ of the form $\left\{e^{1}, e^{2}, a\right\}$ such that the $\mathbb{C}$-valued 1 -form $e=e^{1}+i e^{2}$ annihilates $v$ and is given by

$$
\left(\frac{2 \pi}{\ell_{\gamma}}\right)^{1 / 2} e=d z-2 i(v z+\mu \bar{z}) d t+e,
$$

where the $d t$ and $d z$ components of $e$ are bounded by $c_{0}|z|^{2}$ and the $d \bar{z}$ component by $c_{0}|z|$. Fix such a chart for each Reeb orbit from $\Theta$ so that their images are pairwise disjoint. Such a map is used in what follows to implicitly identify a neighborhood of given Reeb orbit from $\Theta$ with $S^{1} \times D$. In particular, this identification supplies coordinates, $t \in S^{1}$ and $z \in D$, for a neighborhood of the given Reeb orbit.

The constructions that follow require a constant $\rho_{*}>0$ but less than $1 / 100$ times the radius of $D$. An upper bound for the constant $\rho_{*}$ is given in Lemma 3.2. This constant can depend on $r$, but it is important that $r \rho_{*}^{2}>r^{\delta}$ for some fixed $\delta>0$. The construction of instanton solutions to (1-14) takes $\rho_{*}=r^{-1 / 2+3 \sigma}$ where $\sigma \in(0,1 / 100)$ is an appropriately chosen constant.

When $\gamma$ comes from a pair in $\Theta$, use $U_{\gamma} \subset M$ to denote the $\varphi_{\gamma}$-image of the subset $(t, z) \in S^{1} \times D$ with $|z| \leq 4 p_{*}$. Use $U_{\gamma}^{\prime} \subset U_{\gamma}$ to denote the $\varphi_{\gamma}$-image of the concentric solid torus where $|z| \leq \rho_{*}$. The pair $\mathfrak{c}_{\Theta \mathfrak{J}}$ is given first over $U_{0}=M-\operatorname{int}\left(\bigcup_{(\gamma, m) \in \Theta} U_{\gamma}^{\prime}\right)$ and then over each $U_{\gamma}$.

To specify $\mathfrak{c}_{\Theta \mathfrak{J}}$ over $U_{0}$, remark that $E$ 's restriction to $U_{0}$ is isomorphic to the trivial bundle. Choose an isomorphism to identify $\mathbb{S}=E \oplus E K^{-1}$ over $U_{0}$ with $\mathbb{C} \oplus K^{-1}$. Granted this identification, take $\mathfrak{c}_{\Theta \mathfrak{J}}$ on $U_{0}$ to be $\left(A_{I},\left(1_{\mathbb{C}}, 0\right)\right)$ where $A_{I}$ is the trivial connection on the bundle $U_{0} \times \mathbb{C}$ that is defined by the product structure and where $1_{\mathbb{C}}$ is the constant section with value $1 \in \mathbb{C}$. This pair is written as $\left(A_{0}, \psi_{0}\right)$ in what follows.

Step 2 This step sets the stage for the definition of $\mathfrak{c}_{\Theta \mathfrak{J}}$ over each set in $\left\{U_{\gamma}\right\}_{(\gamma, m) \in \Theta}$. To start, remark that there are trivializations of $E$ over any given set from $\left\{U_{\gamma}\right\}_{(\gamma, m) \in \Theta}$ with the following property: The trivializing section, 1, for Step 1's trivialization of $E$ 's restriction to $U_{0}$ appears in the trivialization $\left.E\right|_{U_{\gamma}}=U_{\gamma} \times \mathbb{C}$ as the $S^{1}$-independent function $u=z^{m} /|z|^{m}: S^{1} \times(D-0) \rightarrow S^{1} \subset \mathbb{C}$. Here, and in what follows, $U_{\gamma}$ is implicitly identified by $\varphi_{\gamma}$ with its preimage in $S^{1} \times D$. These transition functions exist because $E$ 's first Chern class is Poincare' dual to $\Gamma$ and $\Gamma$ is the class of the cycle $\sum_{(\gamma, m) \in \Theta} m \gamma$ in $H_{1}(M ; \mathbb{Z})$. What with the coordinate $1-$ forms $d t$ and $d z$ giving a trivialization of $T^{*}\left(S^{1} \times D\right)$, this trivialization of $E$ over $U_{\gamma}$ identifies $S$ over $U_{\gamma}$ with the $\mathbb{C} \oplus \mathbb{C}$ product bundle. As before, the direct sum decomposition 
corresponds to the eigenspaces of Clifford multiplication by $a$. The identification just described is implicit in what follows.

Required next are two rather mundane definitions. For the first, fix once and for all, a smooth, nonincreasing, [0,1]-valued function on $\mathbb{R}$ which is 1 on $(-\infty, 5 / 16]$ and equal to 0 on $[7 / 16, \infty)$. Let $\chi: \mathbb{R} \rightarrow[0,1]$ denote this function. Fix a Reeb orbit $\gamma$ so as to define a function, $\chi_{\gamma}: M \rightarrow[0,1]$ with support near $\gamma$. The latter is defined in terms of the coordinates on $S^{1} \times D$ by $X_{\gamma}=\chi\left(|z| / \rho_{*}\right)$. Here is the second: Given $r \geq 1$, set $r_{\gamma}=\left(\ell_{\gamma} / 2 \pi\right) r$ and let $\widehat{r}_{\gamma}: C \rightarrow C$ denote the map that sends $z$ to $r_{\gamma}^{1 / 2} z$.

Step 3 Digress momentarily, and suppose that $\gamma \subset M$ is any given embedded curve. Fix an embedding, $\varphi$, of $S^{1} \times D$ in $M$ so as to identify a tubular neighborhood of $\gamma$ with the solid torus $S^{1} \times D$ and $\gamma$ with $S^{1} \times 0$. Assume that $\varphi$ 's differential is isometric along $\gamma$. Use $\varphi$ to identify $S^{1} \times D$ with its image, $U$. Fix a trivialization $\left.E\right|_{U}=U \times \mathbb{C}$ as described in Step 2, and use this trivialization to identify a given version of $\mathbb{S}$ over $U$ with $\left(S^{1} \times D\right) \times(\mathbb{C} \oplus \mathbb{C})$.

Let $m$ denote a positive integer and choose some fixed $R \gg 1$. Select a smooth map c: $S^{1} \rightarrow \mathfrak{C}_{m}$. Lift $\mathfrak{c}$ as a pair $(A, \alpha)$ so as to give a smooth map from $S^{1}$ into the space of solutions to (1-4). Choose such a lift so that $\alpha /|\alpha|=z^{m} /|z|^{m}$ for $|z| \geq R$. Introduce the solution $A_{0}$, to denote the solution to the $(A, \alpha)$ version of (2-18). Now let $\zeta$ denote an $L_{1}^{2}$ section of $\mathfrak{c}^{*} T_{1,0} \mathfrak{C}_{m}$ with pointwise small norm. View $\zeta$ as an $L_{1}^{2}$ map from $S^{1}$ into $C^{\infty}(\mathbb{C} ; \mathbb{C} \oplus \mathbb{C})$. Use $\zeta$ to define $\hat{\operatorname{xxp}}_{\mathfrak{c}}(\zeta)$ via (2-9); then use $\mathfrak{c}_{* \zeta}=\left(A^{\zeta}, \alpha^{\zeta}\right)$ as shorthand for this pair. For $r \geq 1$, write $\hat{r}_{\gamma}^{*} A^{\zeta}=A^{\zeta, r}$ and $\hat{r}_{\gamma}^{*} \alpha^{\zeta}=\alpha^{\zeta, r}$; these viewed as an $S^{1}$ dependent pair of connection on the trivial bundle over $\mathbb{C}$ and section of this bundle. Set $A_{0}^{r}=\widehat{r}_{\gamma}^{*} A_{0}$. Also, set $u_{m}=z^{m} /|z|^{m}$.

A pair $\left(A_{\gamma}, \psi_{\gamma}\right)$ of connection on $\left.E\right|_{U}$ and section of $\left.S\right|_{U}=U \times(\mathbb{C} \oplus \mathbb{C})$ is given by

$$
A_{\gamma}=\chi_{\gamma} A_{0}^{r} d t+\chi_{\gamma} A^{\zeta, r}-\left(1-\chi_{\gamma}\right) \bar{u}_{r} d u_{r} \text { and } \psi_{\gamma}=\left(\left(1-\chi_{\gamma}\right) u_{r}+\chi_{\gamma} \alpha^{\zeta, r}, 0\right)
$$

Note that the large $r$ versions of $\left(A_{\gamma}, \psi_{\gamma}\right)$ change with a change of the chosen lift of $\mathfrak{c}$ by $a$ a corresponding gauge transformation if the new lift has $\alpha /|\alpha|=z^{m} /|z|^{m}$ where $|z|>\sqrt{r} R$.

Step 4 The set $\mathfrak{J}$ associates to each $(\gamma, m) \in \Theta$ a smooth map $\mathfrak{c}_{\gamma}: S^{1} \rightarrow \mathfrak{C}_{m}$ and a small normed section, $\zeta_{\gamma}$, of $\mathfrak{c}_{\gamma}^{*} T_{1,0} \mathfrak{C}_{m}$. Use this map to define $\left(A_{\gamma}, \psi_{\gamma}\right)$ on $U_{\gamma}$ as instructed in Step 3. Then $\left\{\left(A_{0}, \psi_{0}\right),\left\{\left(A_{\gamma}, \psi_{\gamma}\right)_{(\gamma, m) \in \Theta}\right\}\right\}$ defines a Hermitian connection on the bundle $E \rightarrow M$ with $L^{2}$ curvature and an $L_{1}^{2}$ section of $\mathbb{S}$. Any given gauge equivalence class of this connection and the spinor is denoted in what follows by $\left(A_{J}, \psi_{J}\right)$. This pair $\left(A_{J}, \psi_{J}\right)$ constitute the promised $\mathfrak{c}_{\Theta_{\mathfrak{J}}}$.

Of interest in what follows is the case where each $\mathfrak{c}_{\gamma}$ is a solution to (1-10). 


\section{3.b The perturbative set up}

Let $\Theta$ and $\mathfrak{J}$ be as described in (3-1). This part of the construction sets the stage for the perturbative techniques that are used to modify a specific version of $\mathfrak{c}_{\Theta \mathfrak{J}}$ so as to obtain the desired solution to (1-10).

The strategy used in what follows is to find a particular set $\mathfrak{J}=\left\{\left(\mathfrak{c}_{\gamma}, \zeta_{\gamma}\right)\right\}_{(\gamma, m) \in \Theta}$ and a pair $(b, \eta) \in L_{1}^{2}\left(M ; i T^{*} M \oplus \mathbb{S}\right)$ so that the large $r$ version of (1-13) is solved by

$$
(A, \psi)=\left(A_{\mathfrak{J}}, \psi_{\mathfrak{J}}\right)+\left((2 r)^{1 / 2} b, \eta\right) .
$$

The pair $(A, \psi)$ will solve the desired version of (1-13) if the triple $\mathfrak{b}=(b, \eta, \phi) \in$ $L_{1}^{2}\left(i T^{*} M \oplus \mathbb{S} \oplus i \mathbb{R}\right)$ solves the system of equations

$$
\begin{gathered}
\text { - } * d b-d \phi-2^{-1 / 2} r^{1 / 2}\left(\psi_{\mathfrak{J}}^{\dagger} \tau \eta+\eta^{\dagger} \tau \psi_{\mathfrak{J}}\right)-2^{-1 / 2} r^{1 / 2} \eta^{\dagger} \tau \eta \\
=-2^{-1 / 2} r^{-1 / 2}\left(B_{A_{\mathfrak{J}}}-r\left(\psi_{\mathfrak{J}}^{\dagger} \tau \psi_{\mathfrak{J}}-i a\right)-\left(i * d \mu+\frac{1}{2} B_{A_{K}}\right)\right) . \\
\text { - } D_{A_{\mathfrak{J}}} \eta+2^{1 / 2} r^{1 / 2}\left(\operatorname{cl}(b) \psi_{\mathfrak{J}}+\phi \psi_{\mathfrak{J}}\right)+2 r^{1 / 2}(\operatorname{cl}(b) \eta+\phi \eta)=-D_{A_{\mathfrak{J}}} \psi_{\mathfrak{J}} . \\
\text { - } * d * b-2^{-1 / 2} r^{1 / 2}\left(\eta^{\dagger} \psi_{\mathfrak{J}}-\psi_{\mathfrak{J}}^{\dagger} \eta\right)=0 .
\end{gathered}
$$

Note that solutions to this equation have $\phi=0$. The component $\phi$ of $\mathfrak{b}$ is introduced for the following reason: The part of the left hand side of (3-5) that is linear in $\mathfrak{b}$ defines a first order operator on $C^{\infty}\left(M ; i T^{*} M \oplus \mathbb{S} \oplus i \mathbb{R}\right)$. This linearized operator is denoted in follows as $\mathfrak{L}_{\mathfrak{c} \Theta \mathfrak{J}}$. This operator is elliptic with the $\phi$ term present, but not so otherwise. More is said about this operator momentarily.

With $\mathfrak{L}_{\mathfrak{c}_{\Theta \mathfrak{J}}}$ understood, (3-5) is written schematically as

$$
\mathfrak{L}_{\mathfrak{c}_{\Theta \mathfrak{J}}} \mathfrak{b}+r^{1 / 2} \mathfrak{b} * \mathfrak{b}-\mathfrak{v}=0,
$$

where $\mathfrak{b} \rightarrow \mathfrak{b} * \mathfrak{b}$ denotes a quadratic, fiber preserving self-map of $i T^{*} M \oplus \mathbb{S} \oplus i \mathbb{R}$. This system of equations has the same formal structure as that given in [7, (2.4)]. Even so, this version has subtleties that are absent from [7] because $\mathfrak{L}_{\mathfrak{c} \Theta \mathfrak{J}}$ has eigenvalues with $O(1)$ norm. By way of contrast, the norms of the eigenvalues of [7]'s version of $\mathfrak{L}_{\mathfrak{c} \Theta \mathfrak{J}}$ have norm at least $\frac{1}{2} r^{1 / 2}$. The $O(1)$ eigenvalue norms that arise here are due to the nontrivial behavior of $\left(A_{\mathfrak{J}}, \psi_{\mathfrak{J}}\right)$ near the Reeb orbits that come from $\Theta$.

The strategy used in what follows to deal with the $O(1)$ eigenvalues is much like that used in the article $\mathrm{Gr} \Rightarrow \mathrm{SW}$ from [5] to handle an analogous situation. The idea is to first project (3-5) onto a subspace that is approximately orthogonal to the eigenvectors of $\mathfrak{L}_{\mathfrak{c} \Theta \mathfrak{J}}$ whose eigenvalues have small absolute value. This is done for a particular choice of the data $\left\{\mathfrak{c}_{\gamma}\right\}$, and then for each $\left\{\zeta_{\gamma}\right\}_{\gamma \in \Theta}$ given that each $\zeta_{\gamma}$ has small $L^{\infty}$ norm. A solution, $\mathfrak{b}$, is found that makes this projection of (3-6) equal to zero. This $\mathfrak{b}$ 
depends smoothly on the data $\left\{\zeta_{\gamma}\right\}_{\gamma \in \Theta}$. The remainder of (3-6) is then viewed as a function of $\left\{\zeta_{\gamma}\right\}_{\gamma \in \Theta}$ and it is argued that there is a unique such collection that makes this function zero. The six steps that follow set up the projected version of (3-6).

Before starting, the reader should take note of the following convention used in the remainder of this subsection and subsequently: What is written as " $c_{0}$ " designates a constant that is greater than 1 and whose value is independent of $r, \mu$, the data $\left\{\zeta_{\gamma}\right\}_{(\gamma, m) \in \Theta}$ and sections of various bundles, principally $i T^{*} M \oplus \mathbb{S} \oplus i \mathbb{R}$. However, $c_{0}$ is allowed to depend on $\left\{\mathfrak{c}_{\gamma}\right\}_{(\gamma, m) \in \Theta}$. In any event, its value can be assumed to increase from appearance to appearance.

Step 1 For the moment, let $A \in \operatorname{Conn}(E)$. With $A$ given, use $\mathbb{H}$ to denote the completion of $C^{\infty}\left(M ; i T^{*} M \oplus \mathbb{S} \oplus i \mathbb{R}\right)$ using the norm whose square is given by

$$
\|\mathfrak{h}\|_{\mathbb{H}}^{2}=\int_{M}|\nabla \mathfrak{h}|^{2}+\frac{1}{4} r \int_{M}|\mathfrak{h}|^{2},
$$

where $\nabla$ is the covariant derivative on $C^{\infty}\left(M ; i T^{*} M \oplus \mathbb{S} \oplus i \mathbb{R}\right)$ that acts as the metric's covariant derivative on the sections of $i T^{*} M$ and $i \mathbb{R}$, and acts as the covariant derivative defined by the connection $A_{K}+2 A$ and the metric's Levi-Civita connection on the sections of $\mathbb{S}$. Let $\mathbb{L}$ to denote the $L^{2}$ completion of $C^{\infty}\left(M ; i T^{*} M \oplus \mathbb{S} \oplus i \mathbb{R}\right)$.

Suppose next that $\psi \in C^{\infty}(M ; \mathbb{S})$. The pair $\mathfrak{c}=(A, \psi)$ together define the operator $\mathfrak{L}_{\mathfrak{c}}$ on $C^{\infty}\left(M ; i T^{*} M \oplus \mathbb{S} \oplus i \mathbb{R}\right)$ that takes any given section $\mathfrak{b}=(b, \eta, \phi)$ to the section whose respective three components are

$$
\begin{array}{ll}
\text { - } & * d b-d \phi-2^{-1 / 2} r^{1 / 2}\left(\psi^{\dagger} \tau \eta+\eta^{\dagger} \tau \psi\right) . \\
\text { - } & D_{A} \eta+2^{1 / 2} r^{1 / 2}(\operatorname{cl}(b) \psi+\phi \psi) . \\
\text { - } & * d * b-2^{-1 / 2} r^{1 / 2}\left(\eta^{\dagger} \psi-\psi^{\dagger} \eta\right) .
\end{array}
$$

This operator is symmetric and it extends as an unbounded, self-adjoint operator on the Hilbert space $\mathbb{L}$ with dense domain $\mathbb{H}$. As such, it has discrete spectrum with finite multiplicities and no accumulation points. The spectrum is unbounded from above and from below.

Certain Sobolev inequalities play a central role in the subsequent analysis. In particular, given that $|\nabla \mathfrak{h}| \geq|d| \mathfrak{h}||$, the standard Sobolev inequalities for $L_{1}^{2}(M ; \mathbb{R})$ guarantee that $\|\cdot\|_{\mathbb{H}}$ dominates the $L^{p}$ norms of $\mathfrak{h}$ for $p \leq 6$. Indeed, if $\mathfrak{h} \rightarrow\|\mathfrak{h}\|_{\mathbb{H}}$ is any norm with the property that $\|\mathfrak{h}\|_{\mathbb{H}} \geq c_{0}\left(\left\|d|\mathfrak{h}|_{2}\right\|+r^{1 / 2}\|\mathfrak{h}\|_{2}\right)$, then

$$
\|\mathfrak{h}\|_{p} \leq c_{0} r^{(p-6) / 4 p}\|\mathfrak{h}\|_{\mathbb{H}}
$$

for any $p \in[2,6]$. 
Step 2 In what follows the space $\mathbb{H}$ is defined by taking $A$ to be the version of $A_{\mathfrak{J}}$ that is defined by the data $\mathfrak{J}_{0}=\left\{\left(\mathfrak{c}_{\gamma}, \zeta_{\gamma}=0\right)\right\}_{(\gamma, m) \in \Theta}$. What is denoted as $\mathfrak{L}_{\mathfrak{c} \Theta \mathfrak{J}}$ in (3-6) differs from its $\mathfrak{c}=\mathfrak{c}_{\Theta \mathfrak{J}_{0}}$ version of (3-8) by a degree zero operator that has the schematic form $\mathfrak{b} \rightarrow \mathfrak{h} * \mathfrak{b}$ where $\mathfrak{h}$ is a bounded, $L_{1}^{2}$ section of $i T^{*} M \oplus \mathbb{S} \oplus i \mathbb{R}$. As a consequence, $\mathfrak{L}_{\mathfrak{c}_{\Theta \mathfrak{J}}}$ defines a bounded, linear map from $\mathbb{H}$ into $\mathbb{L}$. The remainder of this step and the next constitute a digression to describe $\mathfrak{L}_{\mathfrak{c}_{\Theta \mathfrak{J}}}$ in more detail. This is in preparation for an upcoming step where the aforementioned projections in $\mathbb{L}$ and $\mathbb{H}$ are defined.

To start the more detailed description of $\mathfrak{L}_{\mathfrak{c}_{\Theta \mathfrak{J}}}$, note that the identification of $E$ over $U_{0}$ as $U_{0} \times \mathbb{C}$ identifies the operator $\mathfrak{L}_{\mathfrak{c}_{\Theta \mathfrak{J}}}$ in (3-6) on $U_{0}$ with the $\left(A_{I}, \psi_{I}\right)$ version of the operator in (3-8). The latter version of (3-8) is denoted in what follows as $\mathfrak{L}_{I}$. Let $\mathbb{H}_{I}$ denote the completion of $C^{\infty}\left(M ; i T^{*} M \oplus \mathbb{S} \oplus i \mathbb{R}\right)$ using the $A=A_{I}$ version of (3-7). Define $\mathbb{L}_{I}$ to be the corresponding $L^{2}$ completion. As noted in [6, Section 5.5], the operator $\mathfrak{L}_{I}$ defines a bounded, invertible map from $\mathbb{H}_{I}$ to $\mathbb{L}_{I}$. [6, Equation (5.22)] guarantees that

$$
\left\|\mathfrak{L}_{I} \mathfrak{h}\right\|_{2}^{2} \geq\|h\|_{\mathbb{H}}^{2}
$$

when $r$ is large.

Step 3 The identification of any given version of $U_{\gamma}$ with a subset of $S^{1} \times \mathbb{C}$ allows a convenient rewriting of the operator $\mathfrak{L}_{\mathfrak{c} \Theta \mathfrak{J}}$ over $U_{\gamma}$. What follows describes the result.

To start, remark that the trivialization of the bundle $E$ with the associated tubular neighborhood map $\varphi_{\gamma}$ identifies $\mathbb{S}$ with the restriction to $S^{1} \times D$ of the product $\mathbb{C} \oplus \mathbb{C}$ bundle $\left(S^{1} \times \mathbb{C}\right) \times(\mathbb{C} \oplus \mathbb{C})$. This trivialization is such that Clifford multiplication by $d t$ and by $d z$ act on $\mathbb{C} \oplus \mathbb{C}$ along the $z=0$ circle as constant matrices. This identification is used implicitly to view the restriction to $U_{\gamma}$ of a map from $S^{1} \times D$ to $(\mathbb{C} \oplus \mathbb{C})$ as a section of $\mathbb{S}$ over $U_{\gamma}$.

The 1-forms $d t$ and $d z$ also identify $T^{*} M$ on $U_{\gamma}$ with the restriction to $U_{\gamma}$ of the bundle $\left(S^{1} \times D\right) \times(\mathbb{R} \times \mathbb{C})$, this being $T^{*}\left(S^{1} \times D\right)$. The latter trivialization allows the restriction to $S^{1} \times D$ of a section over $S^{1} \times \mathbb{C}$ of $i T^{*}\left(S^{1} \times \mathbb{C}\right)$ to be viewed as a section of $i T^{*} M$ over $U_{\gamma}$. This is also implicit in what follows.

Introduce the $\mathbb{C}$-valued 1 -form $e$ from (3-2) and use $\{e, \bar{e}, a\}$ to write a given $i-$ valued 1 -form $b$ as $q_{3} a+\frac{1}{2}(q \bar{e}-\bar{q} e)$. Given an $i$-valued function $\phi$, introduce $p$ to denote the $\mathbb{C}$-valued function $p=\phi+i q_{3}$. Write a given spinor $\eta$ in two component form $\left(\eta_{0}, \eta_{1}\right)$ with respect to the splitting of $\mathbb{S}$. Then the three components of the first line in (3-8) and the third line in (3-8) can be combined to give the following two 
$\mathbb{C}$-valued expressions:

$$
\begin{array}{ll}
\text { - } & 2\left(\frac{2 \pi}{\ell_{\gamma}}\right)^{1 / 2}\left(-\bar{\partial} p+2^{-1 / 2} r_{\gamma}^{1 / 2} \bar{\psi}_{\gamma 0} \eta_{1}+\frac{i}{2}\left(\frac{2 \pi}{\ell_{\gamma}}\right)^{1 / 2} \frac{\partial}{\partial t} q\right)+\mathfrak{r}_{1} . \\
\text { - } 2\left(\frac{2 \pi}{\ell_{\gamma}}\right)^{1 / 2}\left(\partial q+2^{-1 / 2} r_{\gamma}^{1 / 2} \bar{\psi}_{\gamma 0} \eta_{0}-\frac{i}{2}\left(\frac{2 \pi}{\ell_{\gamma}}\right)^{1 / 2} \frac{\partial}{\partial t} p\right)+\mathfrak{r}_{2} .
\end{array}
$$

Here, $\psi_{\gamma 0}$ is the first component of $\psi_{\gamma}$ from (3-2). Meanwhile $\partial=\partial / \partial z$ and $\bar{\partial}=\partial / \partial \bar{z}$. Finally, the norms of $\mathfrak{r}_{1,2}$ are bounded by $c_{0}|z|(|\nabla q|+|\nabla p|)+c_{0}(|q|+|p|)$. After dividing by the factor 2 , the two $\mathbb{C}$-valued components from the second line of the $\left(A_{\gamma}, \psi_{\gamma}\right)$ version of (3-8) can be written as

$$
\begin{array}{ll}
\text { - } 2\left(\frac{2 \pi}{\ell_{\gamma}}\right)^{1 / 2}\left(-\partial_{A_{\gamma}} \eta_{1}+2^{-1 / 2} r_{\gamma}^{1 / 2} \psi_{\gamma 0} p+\frac{i}{2}\left(\frac{2 \pi}{\ell_{\gamma}}\right)^{1 / 2} \nabla_{t A_{\gamma}} \eta_{0}\right)+\mathfrak{r}_{3}, \\
\text { - } 2\left(\frac{2 \pi}{\ell_{\gamma}}\right)^{1 / 2}\left(\bar{\partial}_{A_{\gamma}} \eta_{0}+2^{-1 / 2} r_{\gamma}^{1 / 2} \psi_{\gamma 0} q-\frac{i}{2}\left(\frac{2 \pi}{\ell_{\gamma}}\right)^{1 / 2} \nabla_{t A_{\gamma}} \eta_{1}\right)+\mathfrak{r}_{4},
\end{array}
$$

where $\partial_{A_{\gamma}}$ here denotes the covariant version of $\partial$ and $\nabla_{t A_{\gamma}}$ denotes the covariant version of $\partial / \partial t$ as defined by the connection $A_{\gamma}$. Here, $\mathfrak{r}_{3,4}$ indicate terms whose norms are bounded by $c_{0}\left|z \| \nabla_{A_{\gamma}} \eta\right|+c_{0}|\eta|$.

What follows describes a useful way to package this. To start, write a section, $\mathfrak{f}$, of $i T^{*} M \oplus \mathbb{S} \oplus i \mathbb{R}$ over $U_{\gamma}$ as $\mathfrak{f}=\left(\left(q, \eta_{0}\right),\left(p, \eta_{1}\right)\right)$ in the manner just described. Written this way, sections of $i T^{*} M \oplus \mathbb{S} \oplus i \mathbb{R}$ over $U_{\gamma}$ are viewed as maps from $S^{1} \times \mathbb{C}$ to $\mathbb{C}^{4}$. Now write $\mathbb{C}^{4}=\mathbb{V}_{0} \oplus \mathbb{V}_{1}$ where $\mathbb{V}_{0}$ and $\mathbb{V}_{1}$ are both copies of $\mathbb{C} \oplus \mathbb{C}$. Then, the $\left(q, \eta_{0}\right)$ part of $\mathfrak{f}$ maps to $\mathbb{V}_{0}$ and the $\left(p, \eta_{1}\right)$ part maps to $\mathbb{V}_{1}$. This identifies a section over $U_{\gamma}$ of $i T^{*} M \oplus \mathbb{S} \oplus i \mathbb{R}$ with a map from the relevant part of $S^{1} \times \mathbb{C}$ to $\mathbb{V}_{0} \oplus \mathbb{V}_{1}$. With this identification understood, then $\mathfrak{L}_{\mathfrak{c}_{\Theta \mathfrak{J}}} \mathfrak{f}$ on $U_{\gamma}$ can be written as the map to $\mathbb{V}_{0} \oplus \mathbb{V}_{1}$ whose component in $\mathbb{V}_{0}$ is

$$
\begin{array}{ll}
-2\left(\frac{2 \pi}{\ell_{\gamma}}\right)^{1 / 2}\left(\left(-\overline{\partial p}+2^{-1 / 2} r_{\gamma}^{1 / 2} \bar{\psi}_{\gamma 0} \eta_{1}\right)+\frac{i}{2}-\left(\frac{2 \pi}{\ell_{\gamma}}\right)^{1 / 2} \frac{\partial}{\partial t} q\right)+\mathfrak{r}_{00}, \\
\text { - } 2\left(\frac{2 \pi}{\ell_{\gamma}}\right)^{1 / 2}\left(\left(-\partial_{A_{\gamma}} \eta_{1}+2^{-1 / 2} r_{\gamma}^{1 / 2} \psi_{\gamma 0} p\right)+\frac{i}{2}\left(\frac{2 \pi}{\ell_{\gamma}}\right)^{1 / 2} \nabla_{t A_{\gamma}} \eta_{0}\right)+\mathfrak{r}_{01},
\end{array}
$$


and whose component in $\mathbb{V}_{1}$ is

$$
\begin{array}{ll}
-2\left(\frac{2 \pi}{\ell_{\gamma}}\right)^{1 / 2}\left(\left(\partial q+2^{-1 / 2} r_{\gamma}^{1 / 2} \bar{\psi}_{\gamma 0} \eta_{0}\right)-\frac{i}{2}\left(\frac{2 \pi}{\ell_{\gamma}}\right)^{1 / 2} \frac{\partial}{\partial t} p\right)+\mathfrak{r}_{10} . \\
\text { - } 2\left(\frac{2 \pi}{\ell_{\gamma}}\right)^{1 / 2}\left(\left(\bar{\partial}_{A_{\gamma}} \eta_{0}+2^{-1 / 2} r_{\gamma}^{1 / 2} \psi_{\gamma 0} q\right)-\frac{i}{2}\left(\frac{2 \pi}{\ell_{\gamma}}\right)^{1 / 2} \nabla_{t A_{\gamma}} \eta_{1}\right)+\mathfrak{r}_{11} .
\end{array}
$$

Here, each $\mathfrak{r}_{* *}$ has norm bounded by $c_{0}(|z||\nabla \mathfrak{f}|+|\mathfrak{f}|)$ where $\nabla$ here denotes the covariant derivative of $\mathfrak{f}$ that acts on the $(q, p)$ components of $\mathfrak{f}$ as the standard derivative operator on $C^{\infty}\left(S^{1} \times \mathbb{C} ; \mathbb{C}\right)$, and on the $\left(\eta_{0}, \eta_{1}\right)$ components of $\mathfrak{f}$ as the covariant derivative on $C^{\infty}\left(S^{1} \times \mathbb{C} ; \mathbb{C}\right)$ that is defined by $A_{\gamma}$.

Step 4 Let $\widehat{\vartheta}_{\gamma, r}$ denote the operator with domain $C^{\infty}\left(S^{1} \times \mathbb{C} ; \mathbb{V}_{0}\right)$ and range space $L^{2}\left(S^{1} \times \mathbb{C} ; \mathbb{V}_{1}\right)$ that sends a given $\left(q, \eta_{0}\right)$ to the map with respective $\mathbb{V}_{1}$ components

$$
\partial q+2^{-1 / 2} r_{\gamma}^{1 / 2} \bar{\alpha}^{\zeta, r} \eta_{0} \text { and } \bar{\partial}_{A, r} \eta_{0}+2^{-1 / 2} r_{\gamma}^{1 / 2} \alpha^{\zeta, r} q
$$

Let $\mathbb{L}_{\gamma \zeta} \subset \mathbb{L}$ denote the subspace of elements $\mathfrak{f}=\chi_{\gamma}\left(\left(q, \eta_{0}\right),(0,0)\right)$ such that $\widehat{\vartheta}_{\gamma, r}\left(q, \eta_{0}\right)=0$ at each $t \in S^{1}$. To elaborate, note that this subspace inclusion uses implicitly the identification just described between $i T^{*} M \oplus \mathbb{S} \oplus i \mathbb{R}$ over $U_{\gamma}$ and $U_{\gamma} \times\left(\mathbb{V}_{0} \oplus \mathbb{V}_{1}\right)$. The subspace $\mathbb{L}_{\gamma \zeta}$ is closed in $\mathbb{L}$. Let $\Pi_{\gamma \zeta}$ denote the $L^{2}$-orthogonal projection from $\mathbb{L}$ to $\mathbb{L}_{\gamma \zeta}$.

Use $\Pi_{\zeta}=\bigoplus_{(\gamma, m) \in \Theta} \Pi_{\gamma \zeta}$. Let $\mathbb{L}_{\zeta}^{\perp} \subset \mathbb{L}$ denote the $L^{2}$ orthogonal complement of $\bigoplus_{(\gamma, m) \in \Theta} \mathbb{L}_{\gamma \zeta}$, thus the kernel of $\Pi_{\zeta}$. The kernel of $\Pi_{\zeta}: \mathbb{H} \rightarrow \bigoplus_{(\gamma, m) \in \Theta} \mathbb{L}_{\gamma \zeta}$ is denoted by $\mathbb{H} \frac{\perp}{\zeta}$.

It follows from Lemma 2.5 that the projection $\Pi_{\zeta}$ maps $\mathbb{H}$ to itself as a bounded operator; thus $\mathbb{H} \frac{\perp}{\zeta}=\left(1-\Pi_{\zeta}\right) \mathbb{H}$.

The next lemma refers to constants $\varepsilon_{0}$ and $\rho_{*}$. The former is defined in Part 8 of Section 2.a, the latter is defined in Step 1 of Section 3.a.

Lemma 3.2 Fix $\sigma \in(0,1 / 2)$ and smooth maps $\left\{\mathfrak{c}_{\gamma}: S^{1} \rightarrow \mathfrak{C}_{m}\right\}_{(\gamma, m) \in \Theta}$. There exists a constant, $\kappa>1+\varepsilon_{0}^{-1}$ with following significance: Suppose that $r \geq \kappa$ and that the constant $\rho_{*}$, obeys $r<\rho_{*}<\kappa^{-1}$. Fix sections $\left\{\zeta_{\gamma} \in L_{1}^{2}\left(S^{1} ; \mathfrak{c}_{\gamma}^{*} T_{1,0} \mathfrak{C}_{m}\right)\right\}(\gamma, m) \in \Theta$ with $L^{\infty}$ norm less than $\kappa^{-1}$ so as to define $\mathfrak{J}$. Then

$$
\kappa^{-1}\|\mathfrak{f}\|_{\mathbb{H}} \leq\left\|\left(1-\Pi_{\zeta}\right) \mathfrak{L}_{\mathfrak{c}_{\Theta \mathfrak{J}}} \mathfrak{f}\right\|_{2} \leq \kappa\|\mathfrak{f}\|_{\mathbb{H}}
$$

for all $f \in \mathbb{H}_{\zeta}^{\frac{\perp}{\zeta}}$. In addition, if each $\zeta_{\gamma}$ has $L_{1}^{2}$ norm less than $\kappa^{-1}$, then $\left(1-\Pi_{\zeta}\right) \mathfrak{L}_{\mathfrak{c}_{\Theta \mathfrak{J}}}$ maps $\mathbb{H}_{\zeta}^{\perp}$ onto $\mathbb{L}_{\zeta}^{\perp}$. Thus, $\left(1-\Pi_{\zeta}\right) \mathfrak{L}_{\mathfrak{c}_{\Theta \mathfrak{J}}}: \mathbb{H}_{\zeta}^{\perp} \rightarrow \mathbb{L}_{\zeta}^{\perp}$ is invertible and its inverse has norm bounded by $\kappa$. 
The proof is given momentarily.

Step 5 To motivate all of this, return now (3-6). It follows from (3-9) that the map $\mathfrak{b} \rightarrow \mathfrak{b} * \mathfrak{b}$ defines a smooth map from $\mathbb{H}$ to $\mathbb{L}$. Thus, what is written on the right hand side of (3-6) defines a smooth map from $\mathbb{H}$ to $\mathbb{L}$. Restrict this map to $\mathbb{H} \frac{\perp}{\zeta}$; then view

$$
\left(1-\Pi_{\zeta}\right)\left(\mathfrak{L}_{\mathfrak{c}_{\Theta \mathfrak{J}}} \mathfrak{b}+r^{1 / 2} \mathfrak{b} * \mathfrak{b}-r^{-1 / 2} \mathfrak{v}\right)=0
$$

as an equation for $\mathfrak{b} \in \mathbb{H} \frac{\perp}{\zeta}$. This is the promised projected version of (3-6). Lemma 3.2 is used to write the latter equation as a fixed point equation for a self-map on $\mathbb{H} \frac{\perp}{\zeta}$; this the map

$$
b \rightarrow \mathbb{T}(b)=\left(\mathfrak{L}^{\perp}\right)^{-1}\left(1-\Pi_{\zeta}\right)\left(-r^{1 / 2} \mathfrak{b} * \mathfrak{b}+r^{-1 / 2} \mathfrak{v}\right),
$$

where $\mathfrak{L}^{\perp}: \mathbb{H}_{\zeta}^{\perp} \rightarrow \mathbb{L}_{\zeta}^{\perp}$ is the restriction to $\mathbb{H}_{\zeta}^{\perp}$ of $\left(1-\Pi_{\zeta}\right) \mathfrak{L}_{\mathfrak{c}_{\Theta \mathfrak{J}}}$. The norm bounds given by Lemma 3.2 are used to prove that $\mathbb{T}$ maps a certain ball in $\mathbb{H}_{\zeta}^{\perp}$ to itself as a contraction mapping. This is explained in the next subsection.

Proof of Lemma 3.2 The upper bound asserted by the lemma is straightforward to prove and so the proof is left to the reader.

The proof of the lower bound starts with a local form of Lemma 3.2. To set the stage for the local version, fix $\gamma \in \Theta$. In what follows, $\mathbb{H}_{\gamma}$ is used to denote the completion of $C^{\infty}\left(S^{1} \times \mathbb{C} ; \mathbb{V}_{0} \oplus \mathbb{V}_{1}\right)$ using the version of (3-7) that replaces $M$ with $S^{1} \times \mathbb{C}$ and has the covariant derivative, $\nabla$, acting as follows on any given $\left(\left(q, \eta_{0}\right),\left(p, \eta_{1}\right)\right)$ : It acts as the exterior derivative $d$ on $q$ and $p$, and acts as the covariant derivative given by the $\zeta=0$ version of $A_{\gamma}$ on $\eta_{0}$ and $\eta_{1}$. In this regard, the norms on $\mathbb{V}_{0}$ and $\mathbb{V}_{1}$ are their vector space norms as $\mathbb{C} \oplus \mathbb{C}$; and integration is defined using the Euclidean volume for $d t \wedge(i / 2)(d z \wedge d \bar{z})$. Use $\mathfrak{L}_{\gamma}$ to denote the analogous $L^{2}$ completion. Use $\mathbb{L}_{\gamma}$ to denote the operator on $C^{\infty}\left(S^{1} \times \mathbb{C} ; \mathbb{V}_{0} \oplus \mathbb{V}_{1}\right)$ whose respective $\mathbb{V}_{0}$ and $\mathbb{V}_{1}$ components are given by the versions of (3-13) and (3-14) that have $\mathfrak{r}_{* *}$ set equal to zero. This operator defines a bounded, linear map from $\mathbb{H}_{\gamma}$ to $\mathbb{L}_{\gamma}$.

Let $\widehat{\vartheta}_{\gamma, r}$ denote for the moment the $\zeta=0$ version of what is defined in (3-15). Define $\mathbb{L}_{\gamma *} \subset \mathbb{L}_{\gamma}$ to be the subspace spanned by elements $\mathfrak{f}=\left(\left(q, \eta_{0}\right),(0,0)\right)$ that obey $\hat{\vartheta}_{\gamma \cdot r}\left(q, \eta_{0}\right)=0$ at each $t \in S^{1}$. This is a closed subspace. Let $\Pi_{\gamma *}$ denote the $L^{2}-$ orthogonal projection from $\mathbb{L}_{\gamma}$ to $\mathbb{L}_{\gamma *}$. Use $\mathbb{L}_{\gamma *}^{\perp} \subset \mathbb{L}_{\gamma}$ to denote the $L^{2}$ orthogonal complement of $\mathbb{L}_{\gamma *}$. The kernel of $\Pi_{\gamma *}: \mathbb{H}_{\gamma} \rightarrow \mathbb{L}_{\gamma *}$ is denoted by $\mathbb{H}_{\gamma^{*}}$. According to Lemma 2.5, the projection $\Pi_{\gamma *}$ maps $\mathbb{H}_{\gamma}$ to itself as a bounded operator; this implies that $\mathbb{H}_{\gamma_{*}}^{\perp}=\left(1-\Pi_{\gamma *}\right) \mathbb{H}_{\gamma}$.

What follows is the promised local version of Lemma 3.2. 
Lemma 3.3 Given a smooth map $\mathfrak{c}_{\gamma}: S^{1} \rightarrow \mathfrak{C}_{m}$, there exists $\kappa \geq 1$ with the following significance: Suppose that $r \geq \kappa$. Use $\mathfrak{c}_{\gamma}$ to construct the pair $\left(A_{\gamma}, \psi_{\gamma}\right)$, the operator $\mathfrak{L}_{\gamma}$ and the projection $\Pi_{\gamma *}$. Then $\left(1-\Pi_{\gamma_{*}}\right) \mathfrak{L}_{\gamma}$ maps $\mathbb{H}_{\gamma^{*}}^{\perp}$ onto $\mathbb{L}_{\gamma^{*}}$. In addition,

$$
\kappa^{-1}\|\mathfrak{f}\|_{\mathbb{H}} \leq\left\|\left(1-\Pi_{\gamma *}\right) \mathfrak{L}_{\gamma} \mathfrak{f}\right\|_{2} \leq \kappa\|\mathfrak{f}\|_{\mathbb{H}}
$$

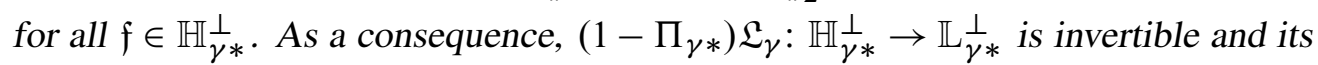
inverse has norm bounded by $\kappa$.

This lemma is proved momentarily. What follows directly explains how it is used to prove Lemma 3.2.

Consider first the claimed lower bound. To this end, write $\mathfrak{L}_{0}$ and $\Pi_{0}$ for the versions of $\mathfrak{L}_{c_{\Theta(\cdot)}}$ and $\Pi_{\zeta}$ that are defined by the data $\left\{\left(\mathfrak{c}_{\gamma}, \zeta_{\gamma}=0\right)\right\}_{\gamma \in \Theta}$. Here are three key facts:

Fact 1 If $\zeta$ has $L^{\infty}$ norm less than $\varepsilon$, then $\left\|\left(\mathfrak{L}_{\mathfrak{c}_{\Theta \mathfrak{J}}}-\mathfrak{L}_{0}\right) \mathfrak{f}\right\|_{2} \leq c_{0} \varepsilon r^{1 / 2}\|\mathfrak{f}\|_{2}$ for all $\mathfrak{f} \in \mathbb{H}$. Likewise, $\left\|\left(\Pi_{\zeta}-\Pi_{0}\right) \mathfrak{f}\right\|_{2} \leq c_{0} \varepsilon\|\mathfrak{f}\|_{2}$ for all $\mathfrak{f} \in \mathbb{L}$. Thus, if $\mathfrak{f} \in \mathbb{H}_{\zeta}^{\perp}$ then $\left\|\Pi_{0} \mathfrak{f}\right\|_{2} \leq c_{0} \varepsilon\|\mathfrak{f}\|_{2}$.

Fact 2 If $\zeta$ has $L^{\infty}$ norm less than $\varepsilon$, then Fact 1 with Lemma 3.2's upper bound imply that $\left\|\left(1-\Pi_{\zeta}\right) \mathfrak{L}_{\mathfrak{c}_{\Theta \mathfrak{J}}} \mathfrak{f}\right\|_{2} \geq c_{0}^{-1}\left\|\left(1-\Pi_{0}\right) \mathfrak{L}_{0} \mathfrak{f}\right\|_{2}-c_{0} \varepsilon\|\mathfrak{f}\|_{\mathbb{H}}$ for all $\mathfrak{f} \in \mathbb{H}$.

Fact 3 Write $\mathfrak{f} \in \mathbb{H}$ as $\mathfrak{f}^{\perp}+\Pi_{0} \mathfrak{f}$. Use the $\zeta=0$ version of the inequality in (2-23) to see that $\left\|\left(1-\Pi_{0}\right) \mathfrak{L}_{0} \Pi_{0} \mathfrak{f}\right\|_{2} \leq c_{0}\|\mathfrak{f}\|_{2}$.

The preceding three facts have the following consequence: If $\zeta$ has $L^{\infty}$ norm less than $\varepsilon$, and if $f \in \mathbb{H} \frac{\perp}{\zeta}$, then $\left\|\left(1-\Pi_{\zeta}\right) \mathfrak{L}_{\mathfrak{c}_{\Theta \mathcal{J}}} \mathfrak{f}\right\|_{2} \geq c_{0}^{-1}\left\|\left(1-\Pi_{0}\right) \mathfrak{L}_{0}\left(1-\Pi_{0}\right) \mathfrak{f}\right\|_{2}-c_{0} \varepsilon\|\mathfrak{f}\|_{\mathbb{H}}$. This understood, it is enough to establish the lemma's lower bound for the case where $\mathfrak{J}$ is the set $\left\{\left(\mathfrak{c}_{\gamma}, \zeta_{\gamma}=0\right)\right\}_{\gamma \in \Theta}$.

To consider the lemma's asserted lower bound in the case $\left\{\left(\mathfrak{c}_{\gamma}, \zeta_{\gamma}=0\right)\right\}_{\gamma \in \Theta}$, note first that Fact 3 above has the counterpart $\left\|\left(1-\Pi_{0}\right) \mathfrak{L}_{0} \mathfrak{f}\right\|_{2} \geq c_{0}^{-1}\left\|\mathfrak{L}_{0} \mathfrak{f}\right\|_{2}-c_{0}\|\mathfrak{f}\|_{2}$ if $\Pi_{0} f=0$. Keeping this in mind, write $\mathfrak{f} \in \mathbb{H}$ as $\chi_{0} \mathfrak{f}+\sum_{(\gamma, m) \in \Theta} \chi_{\gamma} \mathfrak{f}$ where $\chi_{0}=1-\sum_{(\gamma, m) \in \Theta} \chi_{\gamma}$. This done, then

$$
\begin{aligned}
\left\|\mathfrak{L}_{0} \mathfrak{f}\right\|_{2}^{2}=\left\|\mathfrak{L}_{0}\left(\chi_{0} \mathfrak{f}_{0}\right)\right\|_{2}^{2}+\sum_{\gamma \in \Theta}\left\|\mathfrak{L}_{0}\left(\chi_{\gamma} \mathfrak{f}\right)\right\|_{2}^{2} & \\
& +2 \sum_{\gamma \in \Theta}\left\langle\mathfrak{L}_{0}\left(\chi_{0} \mathfrak{f}\right), \mathfrak{L}_{0}\left(\chi_{\gamma} \mathfrak{f}\right)\right\rangle_{2} .
\end{aligned}
$$

Introduce $\mathfrak{f}_{0}$ to denote $\chi_{0} \mathfrak{f}$ and $\mathfrak{f}_{\gamma}$ to denote $\chi_{\gamma} \mathfrak{f}$. It follows from (2-1), (2-2) and (2-12) that

$$
\left(1+c_{0}\right)\left\|\mathfrak{L}_{0} \mathfrak{f}\right\|_{2}^{2} \geq\left\|\mathfrak{L}_{0} \mathfrak{f}_{0}\right\|_{2}^{2}+\sum_{\gamma \in \Theta}\left\|\mathfrak{L}_{0} \mathfrak{f}_{\gamma}\right\|_{2}^{2}-c_{0} \rho_{*}^{-2}\|\mathfrak{f}\|_{2}^{2} .
$$


Now note that $\mathfrak{L}_{0}=\mathfrak{L}_{I}$ on the support of $\mathfrak{f}_{0}$, and with the identifications given above, it is very nearly $\mathbb{L}_{\gamma}$ on the support of $\mathfrak{f}_{\gamma}$. In particular, (3-13), (3-14) and (3-19) imply that

$$
c_{0}\left\|\mathfrak{L}_{0} \mathfrak{f}\right\|_{2}^{2} \geq\left\|\mathfrak{L}_{I} \mathfrak{f}_{0}\right\|_{2}^{2}+\sum_{\gamma \in \Theta}\left\|\mathfrak{L}_{\gamma} \mathfrak{f}_{\gamma}\right\|_{2}^{2}-c_{0}\left(\rho_{*}^{-2}\|\mathfrak{f}\|_{2}^{2}+\rho_{*}^{2}\|\mathfrak{f}\|_{\mathbb{H}}^{2}\right) .
$$

To continue, write $\mathfrak{f}_{\gamma}=\mathfrak{f}_{\gamma}^{\perp}+\Pi_{\gamma *} \mathfrak{f}_{\gamma}$, where $\mathfrak{f}_{\gamma}^{\perp} \in \mathbb{H} \frac{\perp}{\gamma^{*}}$. A first point to note is

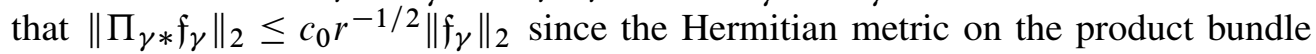
$U_{\gamma} \times\left(\mathbb{V}_{0} \oplus \mathbb{V}_{1}\right)$ is very close to that induced from $M$. This $L^{2}$ inequality also uses (2-1), (2-2) and (2-12), and assumes that $r>c_{0}$. These same facts with Lemma 2.5 imply a similar inequality for the respective derivatives of $\Pi_{\gamma * \mathfrak{f}_{\gamma}}$ and $\mathfrak{f}_{\gamma}$; and thus that $\left\|\Pi_{\gamma *} \mathfrak{f}_{\gamma}\right\|_{\mathbb{H}} \leq c_{0} r^{-1 / 2}\left\|\mathfrak{f}_{\gamma}\right\|_{\mathbb{H}}$.

With the preceding understood, it follows from Lemma 2.5 and Lemma 3.3 that

$$
\left\|\mathfrak{L}_{\gamma} \mathfrak{f}_{\gamma}\right\|_{2}^{2} \geq c_{0}^{-1}\left\|\mathfrak{f}_{\gamma}\right\|_{\mathbb{H}}^{2}
$$

provided that $r>c_{0}$. Meanwhile, (3-10) asserts that $\left\|\mathfrak{L}_{I} \mathfrak{f}_{0}\right\|_{2}^{2} \geq\left\|\mathfrak{f}_{0}\right\|_{\mathbb{H}}^{2}$. Thus, (3-20) and (3-21) imply the lower bound $\left\|\left(1-\Pi_{0}\right) \mathfrak{L}_{0} \mathfrak{f}\right\|_{2} \geq c_{0}^{-1}\|\mathfrak{f}\|_{\mathbb{H}}$ if $r>c_{0}$ and if $\Pi_{0} \mathfrak{f}=0$.

Now consider the assertion that $\left(1-\Pi_{\zeta}\right) \mathfrak{L}_{\mathfrak{c}_{\Theta \mathfrak{J}}}$ maps $\mathbb{H}_{\zeta}^{\perp}$ onto $\mathbb{L}_{\zeta}^{\frac{\perp}{\zeta}}$. As is explained directly, such is the case if both the $L_{1}^{2}$ norm and the $L^{\infty}$ norm of $\zeta$ is bounded by $c_{0}^{-1}$, and if $\left(1-\Pi_{0}\right) \mathfrak{L}_{0}$ maps $\mathbb{H}_{\zeta=0}^{\perp}$ onto $\mathbb{L}_{\zeta=0}^{\perp}$. To see why such is the case, suppose that $\varepsilon>0$ and that each $\zeta_{\gamma}$ now has both $L^{\infty}$ and $L_{1}^{2}$ norm less than $\varepsilon_{0}$. Suppose that $v \in \mathbb{L}_{\zeta}^{\perp}$ is orthogonal to the image via $\left(1-\Pi_{\zeta}\right) \mathfrak{L}_{\mathfrak{c}_{\Theta \mathfrak{J}}}$ of $\mathbb{H}_{\zeta}^{\perp}$, but assume that there exists $\mathfrak{g} \in \mathbb{H} \frac{\perp}{\zeta=0}$ such that $\left(1-\Pi_{0}\right) \mathfrak{L}_{0} \mathfrak{g}=\left(1-\Pi_{0}\right) \mathfrak{v}$. Granted this last assumption, it follows from Fact 1 above that

$$
\left\langle\mathfrak{L}_{\mathfrak{c}_{\Theta \mathfrak{J}}} \mathfrak{g}, \mathfrak{v}\right\rangle_{2} \geq c_{0}^{-1}\|\mathfrak{v}\|_{2}^{2}-c_{0} \varepsilon\|\mathfrak{g}\|_{\mathbb{H}}\|\mathfrak{v}\|_{2}
$$

if the $L^{\infty}$ norm of $\zeta$ is bounded by $\varepsilon$. Given what has been proved so far of Lemma 3.2 , it follows from the equation $\left(1-\Pi_{0}\right) \mathfrak{L}_{0} \mathfrak{g}=\left(1-\Pi_{0}\right) \mathfrak{v}$ that $\|\mathfrak{g}\|_{\mathbb{H}} \leq c_{0}\|\mathfrak{v}\|_{2}$. Thus, (3-22) implies that $\left\langle\mathfrak{L}_{\mathfrak{c} \Theta \mathfrak{J}} \mathfrak{g}, \mathfrak{v}\right\rangle_{2} \geq c_{0}^{-1}\|\mathfrak{v}\|_{2}^{2}$ if $\varepsilon \leq c_{0}^{-1}$. Meanwhile, it follows from Lemma 2.5 that $\left\|\Pi_{\zeta} \mathfrak{g}\right\|_{\mathbb{H}} \leq c_{0} \varepsilon\|g\|_{\mathbb{H}}$ if the $L_{1}^{2}$ norm of $\zeta$ is less than $\varepsilon$ also. If such is the case, then (3-22) implies that $\left\langle\mathfrak{L}_{\mathfrak{c}_{\Theta \mathfrak{J}}}\left(1-\Pi_{\zeta}\right) \mathfrak{g}, \mathfrak{v}\right\rangle_{2} \geq c_{0}^{-1}\|\mathfrak{v}\|_{2}^{2}$ when $\varepsilon \leq c_{0}^{-1}$. Given that $\mathfrak{v}$ is orthogonal to the image of $\mathbb{H}_{\zeta}^{\perp}$ via $\left(1-\Pi_{\zeta}\right) \mathfrak{L}_{\mathfrak{c}_{\Theta \mathfrak{J}}}$, this implies that $\mathfrak{v}=0$.

Granted all of this it is sufficient to prove that $\left(1-\Pi_{0}\right) \mathfrak{L}_{0}$ maps $\mathbb{H}_{\zeta=0}^{\perp}$ onto $\mathbb{L}_{\zeta=0}^{\perp}$. To do so, let $x_{\gamma}$ denote the characteristic function for the support of $\chi_{\gamma}$. Let $\mathfrak{v}$ be as in the preceding paragraph. Interpret $x_{\gamma} \mathfrak{v}$ as an element in $\mathfrak{L}_{\gamma}$. This done, then Lemma 3.3 finds an element $\mathfrak{f}_{\gamma} \in \mathbb{H} \frac{\perp}{\gamma^{*}}$ with $\left(1-\Pi_{\gamma *}\right) \mathfrak{L}_{\gamma} \mathfrak{f}_{\gamma}=\left(1-\Pi_{\gamma *}\right) x_{\gamma} \mathfrak{v}$. Let $x_{0}$ denote 
the characteristic function for the support of $\chi_{0}$. As $\mathfrak{L}_{I}$ is self-adjoint, it follows from (3-10) that there exists $\mathfrak{f}_{0} \in \mathbb{H}_{0}$ with $\mathfrak{L}_{I} \mathfrak{f}_{0}=x_{0} \mathfrak{v}$.

Let $\mathfrak{f}=\chi_{0} \mathfrak{f}_{0}+\sum_{(\gamma, m) \in \Theta} \chi_{\gamma} \mathfrak{f}_{\gamma}$. Given (3-13) and (3-14), it follows that

$$
\left\langle\mathfrak{L}_{\mathfrak{c}_{\Theta \mathfrak{J}} \mathfrak{f}} \mathfrak{f} \mathfrak{v}\right\rangle_{2} \geq c_{0}^{-1}\|\mathfrak{v}\|_{2}^{2}-c_{0}\left(\rho_{*}^{-2}\|\mathfrak{f}\|_{2}^{2}+\rho_{*}^{2}\|\mathfrak{f}\|_{\mathbb{H}}^{2}\right) .
$$

Meanwhile, $\|\mathfrak{f}\|_{\mathbb{H}} \leq c_{0}\left(\left\|\mathfrak{f}_{0}\right\|_{\mathbb{H}}+\sum_{(\gamma, m) \in \Theta}\left\|\mathfrak{f}_{\gamma}\right\|_{\mathbb{H}}\right)$ and so the norm lower bounds given by (3-10) and by Lemma 3.3 imply that $\|\mathfrak{f}\|_{\mathbb{H}} \leq c_{0}^{-1}\|\mathfrak{v}\|_{2}$ when $r \geq c_{0}$. Thus (3-23) implies that

$$
\left\langle\mathfrak{L}_{\mathfrak{c}_{\Theta \mathfrak{J}}} \mathfrak{f}, v\right\rangle_{2} \geq c_{0}^{-1}\|\mathfrak{v}\|_{2}^{2}
$$

when $r \geq c_{0}$.

Now (3-24) does not quite finish things because $\mathfrak{f}$ is not apriori in $\mathbb{H} \frac{\perp}{\zeta}$. Even so, it is a consequence of (2-1), (2-2), (2-12) and Lemma 2.5 that there exists $f^{\prime} \in \mathbb{H} \frac{\perp}{\xi}$ such that $\left\|\mathfrak{f}-\mathfrak{f}^{\prime}\right\|_{\mathbb{H}} \leq c_{0} r^{-1 / 2}\|\mathfrak{f}\|_{\mathbb{H}}$ when $r \geq c_{0}$. Since $\|\mathfrak{f}\|_{\mathbb{H}} \leq c_{0}\|\mathfrak{v}\|_{2}$, it follows from (3-24) that $\left\langle\mathfrak{L}_{\mathfrak{c}_{\Theta \mathfrak{J}}} \mathfrak{f}^{\prime}, \mathfrak{v}\right\rangle_{2} \geq c_{0}^{-1}\|\mathfrak{v}\|_{2}^{2}$. This last inequality can hold only if $\mathfrak{v}=0$.

Proof of Lemma 3.3 The upper bound for $\left\|\left(1-\Pi_{\gamma *}\right) \mathfrak{L}_{\gamma} \mathfrak{f}\right\|_{2}$ is straightforward to prove and left to the reader. Consider the existence of a constant $\kappa$ for which the asserted lower bound inequality is obeyed. To find $\kappa$, look first at the inequality for $\mathfrak{f}=\left((0,0),\left(p, \eta_{1}\right)\right)$. Use $\lambda$ to denote the pair $\left(p, \eta_{1}\right)$. The square of the $L^{2}$ norm of $\mathfrak{L}_{\gamma} \mathfrak{f}$ obeys

$$
\left\|\left(1-\Pi_{\gamma *}\right) \mathfrak{L}_{\gamma} \mathfrak{f}\right\|_{2}^{2}=\frac{2 \pi}{\ell_{\gamma}}\left(\left\|\nabla_{t \gamma} \lambda\right\|_{2}^{2}+4\left\|\hat{\vartheta}_{\gamma, r}^{\dagger} \lambda\right\|_{2}^{2}\right)
$$

A rescaling of the coordinate along the $\mathbb{C}$ factor of $S^{1} \times \mathbb{C}$ and an appeal to (2-8) find that

$$
\left\|\hat{\vartheta}_{\gamma, r}^{\dagger} \lambda\right\|_{2}^{2} \geq c_{0}^{-1}\left(\left\|\nabla_{\gamma}^{\mathbb{C}} \lambda\right\|_{2}^{2}+r_{\gamma}\|\lambda\|_{2}^{2}\right)
$$

here $\nabla_{\gamma}^{\mathbb{C}}$ denotes the covariant derivative in directions tangent to the $\mathbb{C}$ factor of $S^{1} \times \mathbb{C}$. The lemma's norm inequality for $\mathfrak{f}=\left((0,0),\left(p, \eta_{1}\right)\right)$ with a suitable $\kappa$ follows from these last two inequalities.

Consider next the asserted norm inequality when $\mathfrak{f}=\left(\left(q, \eta_{0}\right),(0,0)\right)$. To this end, set $\lambda=\left(q, \eta_{0}\right)$ and note that

$$
\left\|\left(1-\Pi_{\gamma *}\right) \mathfrak{L}_{\gamma} \mathfrak{f}\right\|_{2}^{2} \geq \frac{2 \pi}{\ell_{\gamma}}\left(\left\|\left(1-\Pi_{\gamma *}\right) \nabla_{t \gamma} \lambda\right\|_{2}^{2}+4\left\|\hat{\vartheta}_{\gamma, r} \lambda\right\|_{2}^{2}\right)
$$


Now suppose that $\lambda$ is $L^{2}$-orthogonal on each constant $t \in S^{1}$ slice of $S^{1} \times \mathbb{C}$ to the kernel of $\hat{\vartheta}_{\gamma, r}$. Then (2-8) guarantees that $\left\|\hat{\vartheta}_{\gamma, r} \lambda\right\|_{2}^{2}$ is also larger than what is written on the right hand side of (3-26). Meanwhile, it follows from Lemma 2.5 that

$$
\left\|\left(1-\Pi_{\gamma *}\right) \nabla_{t \gamma} \lambda\right\|_{2} \geq \frac{1}{2}\left\|\nabla_{t \gamma} \lambda\right\|_{2}-c_{0}\|\lambda\|_{2},
$$

where $c_{0}$ depends only on $\mathfrak{c}_{\gamma}$. Granted these facts, the lemma's norm inequality also holds for a suitable $\kappa$ when $\mathfrak{f}=\left(\left(q, \eta_{0}\right),(0,0)\right)$.

Finally, consider $\mathfrak{f}=\mathfrak{f}_{0}+\mathfrak{f}_{1}$ where $\mathfrak{f}_{0}$ is of the form $\left(\left(q, \eta_{0}\right),(0,0)\right)$ and $\mathfrak{f}_{1}$ is of the form $\left((0,0),\left(p, \eta_{1}\right)\right)$. An integration by parts finds that

$$
\begin{aligned}
\left\|\left(1-\Pi_{\gamma *}\right) \mathfrak{L}_{\gamma}\left(\mathfrak{f}_{0}+\mathfrak{f}_{1}\right)\right\|_{2}^{2} \geq \frac{1}{2}\left(\left\|\left(1-\Pi_{\gamma *}\right) \mathfrak{L}_{\gamma} \mathfrak{f}_{0}\right\|_{2}^{2}\right. \\
\left.+\left\|\left(1-\Pi_{\gamma *}\right) \mathfrak{L}_{\gamma} \mathfrak{f}_{1}\right\|_{2}^{2}\right)-c_{0}\|\mathfrak{f}\|_{2}^{2},
\end{aligned}
$$

where $c_{0}$ again depends only on $\mathfrak{c}_{\gamma}$. Given that there exists $\kappa$ that makes the norm inequality true separately for $\mathfrak{f}_{0}$ and $\mathfrak{f}_{1}$, this last inequality implies $\kappa$ can be found to make the lemma's claim true in the general case.

What follows explains why $\kappa$ can be chosen so as to guarantee that $\left(1-\Pi_{\gamma *}\right) \mathfrak{L}_{\gamma}$ is onto. To start, note that $\Pi_{\gamma *} \mathfrak{L}_{\gamma}: \mathbb{H}_{\gamma_{*}}^{\perp} \rightarrow \mathbb{L}_{\gamma *}$ extends as a bounded operator from $\mathbb{L}_{\gamma^{*}}^{\perp}$ to $\mathbb{L}_{\gamma *}$. This understood, suppose that $\mathfrak{f} \in \mathbb{L}_{\gamma^{*}}^{\perp}$ is in the cokernel of $\left(1-\Pi_{\gamma *}\right) \mathfrak{L}_{\gamma}$. If such is the case, then $\left\langle\mathfrak{L}_{\gamma p}, \mathfrak{f}\right\rangle_{2}=\left\langle\Pi_{\gamma *} \mathfrak{L}_{\gamma} \mathfrak{p}, \mathfrak{f}\right\rangle_{2}$ for all $\mathfrak{p} \in \mathbb{H}_{\gamma *}$. From what was just said, this implies that $\left|\left\langle\mathfrak{L}_{\gamma} \mathfrak{p}, \mathfrak{f}\right\rangle_{2}\right| \leq c_{0}\|\mathfrak{p}\|_{2}\|\mathfrak{f}\|_{2}$ for all $\mathfrak{p} \in \mathbb{H}_{\gamma *}$. Since $\mathbb{H}_{\gamma *}$ is dense in $L^{2}$, this implies that $\mathfrak{f}$ is in the domain of the formal, $L^{2}$ adjoint of $\mathfrak{L}_{\gamma}$. Since this version of $\mathfrak{L}_{\gamma}$ is symmetric, so $\mathfrak{f}$ is in $\mathbb{H}_{\gamma_{*}}^{\perp}$. Granted that such is the case, the lemma's norm inequality implies that $\mathfrak{f}=0$.

\section{3.c The right hand side of (3-5)}

The perturbative scheme use here to solve (3-16) and then (3-17) requires that what is written as $\mathfrak{v}$ in the latter have suitably small norm. This understood, this subsection provides bounds on the norms of $B_{A_{\mathfrak{J}}}-r\left(\psi_{J}^{\dagger} \tau \psi_{\mathfrak{J}}-i a\right)$ and $D_{A_{\mathfrak{J}}} \psi_{\mathfrak{J}}$.

By construction, $B_{A_{\mathfrak{J}}}-r\left(\psi_{\mathfrak{J}}^{\dagger} \tau \psi_{\mathfrak{J}}-i a\right)=0$ and $D_{A_{\mathfrak{J}}} \psi_{\mathfrak{J}}=0$ on $U_{0}$. The next lemma describes their behavior on a given $\gamma \in \Theta$ version of $U_{\gamma}$. The lemma refers to the $\mathbb{C}$-valued functions $x_{\zeta}^{r}$ and $\iota_{\zeta}^{r}$ on $S^{1} \times \mathbb{C}$. These are the respective pullbacks via the map $\widehat{r}_{\gamma}$ of the functions $x_{\zeta}$ and $\iota_{\zeta}$ as defined in (2-21). The lemma also refers to the function $f_{\zeta}$ whose square is given in (2-20). In addition, the lemma reintroduces the complex 1-form $e$ from (3-2). Recall that the real and imaginary parts of $e$ with the contact form a define an orthonormal basis for $T^{*} M$ near $\gamma$. 
Lemma 3.4 Given a smooth map $\mathfrak{c}_{\gamma}: S^{1} \rightarrow \mathfrak{C}_{m}$, there is a constant $\kappa \geq 1$ with the following significance: Take $r \geq \kappa$. Fix a section $\zeta_{\gamma}$ of $\mathfrak{c}_{\gamma}^{*} T_{1,0} \mathfrak{C}_{m}$ with $L_{1}^{2}$ and pointwise norm less than $\kappa$. Use $\left(\mathfrak{c}_{\gamma}, \zeta_{\gamma}\right)$ to define $\left(A_{\gamma}, \psi_{\gamma}\right)$. Then $r^{-1 / 2}\left(B_{A_{\gamma}}-r\left(\psi_{\gamma}^{\dagger} \tau \psi_{\gamma}-i a\right)\right)$ and the two components of $D_{A_{\gamma}} \psi_{\gamma}$ can be written as

$$
\begin{aligned}
-\frac{2 \pi}{\ell_{\gamma}}\left(\left(i 2^{-1 / 2} x_{\zeta}^{r}-r_{\gamma}^{1 / 2}(v z+\right.\right. & \left.\mu \bar{z})\left(1-\left|\alpha^{\zeta, r}\right|^{2}\right)\right) \bar{e}, \\
& \left.+\left(i 2^{-1 / 2} \bar{x}_{\zeta}^{r}+r_{\gamma}^{1 / 2}(v \bar{z}+\bar{\mu} z)\left(1-\left|\alpha^{\zeta, r}\right|^{2}\right)\right) e+\mathfrak{z}\right),
\end{aligned}
$$

- $\frac{2 \pi}{\ell_{\gamma}}\left(i \iota_{\zeta}^{r}-2(v z+\mu \bar{z}) \partial_{A^{\zeta, r}} \alpha^{\zeta, r}+\mathfrak{z}_{0}, \mathfrak{z}_{1}\right)$,

where $|\mathfrak{z}|+\left|\mathfrak{z}_{0}\right|+\left|\mathfrak{z}_{1}\right| \leq \kappa r^{-1 / 2}\left(1+f_{\zeta}\right) e^{-\sqrt{r}|z| / 2}$.

The pair $\left(A_{\gamma}, \psi_{\gamma}\right)$ can be constructed as directed above when $\gamma$ is an embedded curve and not a Reeb orbit. In this case, the terms indicated by $\mathfrak{z}$ and $\left(\mathfrak{z}_{0}, \mathfrak{z}_{1}\right)$ in a corresponding version of Lemma 3.4 would have contributions with norm bounded below by $\kappa_{c}^{-1} r^{1 / 2} e^{-\sqrt{r}|z| / 2}$ and bounded above by $\kappa_{c} r^{1 / 2} e^{-\sqrt{r}|z| / 2}$. The fact that these larger contributions are zero if and only if $\gamma$ is a Reeb orbit gives some indication as to how the Seiberg-Witten equations distinguish the Reeb orbits from embedded curves.

Proof of Lemma 3.4 An appeal to (2-1), (2-19) and (2-22) bounds the pointwise norms of $B_{A_{\gamma}}-r\left(\psi_{\gamma}^{\dagger} \tau \psi_{\gamma}-i a\right)$ and $D_{A_{\gamma}} \psi_{\gamma}$ where $d \chi_{\gamma} \neq 0$ by $c_{\mathfrak{c}} r^{-1 / 2}\left(1+f_{\zeta}\right)$ $\times \exp \left(-\frac{1}{2} r^{1 / 2}|z|\right)$. Thus, what is claimed by the lemma holds on the support of $d \chi_{\gamma}$ given that $\rho_{*}^{2} r>r^{\delta}$ with $\delta>0$.

Consider next $B_{A_{\gamma}}-r\left(\psi_{\gamma}^{\dagger} \tau \psi_{\gamma}-i a\right)$ where $\chi_{\gamma}=1$. The curvature 2-form of the connection $A_{\gamma}$ is $\frac{1}{2} r_{\gamma}\left(1-\left|\alpha^{\zeta, r}\right|^{2}\right) d z \wedge d \bar{z}-2^{-1 / 2} r_{\gamma}^{1 / 2}\left(x_{\zeta}^{r} d \bar{z}-\bar{x}_{\zeta}^{r} d z\right) \wedge d t$. Write this 2form in terms of the orthonormal basis given by a and the real and imaginary parts of $e$. Given (2-19), it follows that $B_{A_{\gamma}}$ differs by less than $c_{\mathfrak{c}}\left(r|z|^{2}\left(1-\left|\alpha^{\zeta, r}\right|^{2}\right)+r^{1 / 2}|z|\left|x_{\zeta}^{r}\right|\right)$ from

$$
\begin{aligned}
-i r\left(1-\left|\alpha^{\zeta, r}\right|^{2}\right) \hat{a}+ & \frac{2 \pi}{\ell_{\gamma}} r^{1 / 2}\left(i 2^{-1 / 2} x_{\zeta}^{r}-r_{\gamma}^{1 / 2}(v z+\mu \bar{z})\left(1-\left|\alpha^{\zeta \cdot r}\right|^{2}\right)\right) \bar{e} \\
+ & \frac{2 \pi}{\ell_{\gamma}} r^{1 / 2}\left(i 2^{-1 / 2} \bar{x}_{\zeta}^{r}+r_{\gamma}^{1 / 2}(\nu \bar{z}+\bar{\mu} z)\left(1-\left|\alpha^{\zeta \cdot r}\right|^{2}\right)\right) e .
\end{aligned}
$$

Meanwhile, $r\left(\psi_{\gamma}^{\dagger} \tau \psi_{\gamma}-i a\right)$ is equal to $-i r\left(1-\left|\alpha^{r}\right|^{2}\right) a$. This last point with (3-30) gives the first bullet point of the lemma. The verification of the assertion about $D_{A_{\gamma}} \psi_{\gamma}$ is proved by writing out the Dirac operator using the coordinates $(t, z)$ for $S^{1} \times \mathbb{C}$ and invoking (3-30). This task is straightforward and so left to the reader. 


\section{3.d Solving (3-16)}

The purpose of this subsection is to describe a solution to (3-16). As noted above, the strategy is to view (3-16) as the equation for a fixed point in $\mathbb{H}_{\zeta}^{\perp}$ of the map $\mathbb{T}$ given in (3-17).

Lemma 3.5 Fix $\sigma \in(0,1 / 2)$, a set $\Theta$ as in (3-1) and a set $\left\{\mathfrak{c}_{\gamma} \in C^{\infty}\left(S^{1} ; \mathfrak{C}_{m}\right)\right\}_{(\gamma, m) \in \Theta}$. There exists a constant $\kappa \geq 1$ with the following significance: For each $(\gamma, m) \in \Theta$, fix $\zeta_{\gamma} \in L_{1}^{2}\left(S^{1}, \mathfrak{c}_{\gamma}^{*} T_{1,0} \mathfrak{C}_{m}\right)$ such that $\left|\zeta_{\gamma}\right| \leq \kappa^{-1}$. Fix $r \geq \kappa$ and $\rho_{*} \in\left(r^{-\sigma}, \kappa^{-1}\right)$. Use $\mathfrak{J}=\left\{\left(\mathfrak{c}_{\gamma}, \zeta_{\gamma}\right)\right\}_{(\gamma, m) \in \Theta}$ to define the space $\mathbb{H}_{\xi}^{\perp}$ and the operator $\mathfrak{L}^{\perp}$ that appears in (3-16) and (3-17). Suppose that $\mathfrak{v}$ is a given element $\mathbb{L}$ with $\|\mathfrak{v}\|_{2} \leq \kappa_{c}^{-1} r^{-1 / 4}$. Then the map $\mathbb{T}$ as depicted in (3-17) has a unique fixed point in $\mathbb{H} \frac{\perp}{\zeta}$ with norm less than $\kappa_{c}^{-1} r^{-1 / 4}$. The norm of this fixed point is, in, less than $\kappa_{c}\|\mathfrak{v}\|_{2}$.

Proof of Lemma 3.5 The operation $*$ that appears in (3-17) obeys

$$
\left\|\mathfrak{h} * \mathfrak{h}^{\prime}\right\|_{2} \leq c_{0}\|\mathfrak{h}\|_{L^{4}}\left\|\mathfrak{h}^{\prime}\right\|_{L^{4}} \leq c_{0} r^{-1 / 4}\|\mathfrak{h}\|_{\mathbb{H}}\left\|\mathfrak{h}^{\prime}\right\|_{\mathbb{H}}
$$

This follows from (3-9). Meanwhile, Lemma 3.2 finds an $r$-independent constant $c_{0} \geq 1$ with the property that if $r \geq c_{0}$ then $\left\|\left(\mathfrak{L}^{\perp}\right)^{-1} \mathfrak{w}\right\|_{\mathbb{H}} \leq c_{0}\|\mathfrak{w}\|_{2}$ for any $\mathfrak{w} \in \mathbb{L}^{\perp}$. Granted these last three observations, it follows that

$$
\|\mathbb{T}(\mathfrak{h})\|_{\mathbb{H}} \leq c_{0}\left(\|\mathfrak{v}\|_{2}+r^{1 / 4}\|\mathfrak{h}\|_{\mathbb{H}}^{2}\right) .
$$

In addition,

$$
\left\|\mathbb{T}\left(\mathfrak{h}-\mathfrak{h}^{\prime}\right)\right\|_{\mathbb{H}} \leq c_{0} r^{1 / 4}\left(\|\mathfrak{h}\|_{\mathbb{H}}+\left\|\mathfrak{h}^{\prime}\right\|_{\mathbb{H}}\right)\left\|\mathfrak{h}-\mathfrak{h}^{\prime}\right\|_{\mathbb{H}}
$$

The inequality in (3-32) implies the existence of a constant, $c_{1}>1$ such that $\mathbb{T}$ maps the ball in $\mathbb{H}_{\zeta}^{\perp}$ of radius $c_{1}^{-1} r^{-1 / 4}$ to itself provided that $r \geq c_{1}$, that $\|\mathfrak{v}\|_{2} \leq c_{1}^{-2} r^{1 / 4}$. The inequality in (3-33) implies that such a constant can be chosen as to insure that $\mathbb{T}$ is a contraction mapping on the radius $c_{1}^{-1} r^{-1 / 4}$ ball in $\mathbb{H}_{\zeta} \frac{\perp}{\zeta}$.

Granted the preceding, the contraction mapping theorem asserts that $\mathbb{T}$ has a unique fixed point in the radius $c_{0} r^{-1 / 4}$ ball in $\mathbb{H}_{\zeta}^{\perp}$, and that the norm of this fixed point is bounded by $c_{0}\|\mathfrak{v}\|_{2}$ if $\|\mathfrak{v}\|_{2}$ is smaller than $c_{0}^{-1} r^{-1 / 4}$.

With Lemma 3.5 in hand, consider now the term that is designated by $\mathfrak{v}$ when (3-17) comes from (3-5). By construction, any fixed point of $\mathbb{T}$ is a solution in $\mathbb{H} \frac{\perp}{\zeta}$ to the Equation (3-16). 
Lemma 3.6 Fix $\sigma \in(0,1 / 2)$, a set $\Theta$ as in (3-1), and a set $\left\{\mathfrak{c}_{\gamma} \in C^{\infty}\left(S^{1} ; \mathfrak{C}_{m}\right)\right\}_{(\gamma, m) \in \Theta}$. There exists a constant $\kappa \geq 1$ with the following significance: For each $(\gamma, m) \in \Theta$, fix $\zeta_{\gamma} \in L_{1}^{2}\left(S^{1}, \mathfrak{c}_{\gamma}^{*} T_{1,0} \mathfrak{C}_{m}\right)$ with $L_{1}^{2}$ norm is bounded by $\kappa^{-1}$. Fix $r \geq \kappa$ and $\rho * \in$ $\left(r^{-\sigma}, \kappa^{-1}\right)$. Use $\mathfrak{J}=\left\{\left(\mathfrak{c}_{\gamma}, \zeta_{\gamma}\right)\right\}_{(\gamma, m) \in \Theta}$ to define the space $\mathbb{H} \frac{\perp}{\zeta}$ and the operator $\mathfrak{L}^{\perp}$ that appears in (3-16) and (3-17). Take the term $\mathfrak{v}$ in (3-16) and(3-17) to correspond to what appears on the right hand side of (3-5). Then $\|\mathfrak{v}\|_{2} \leq \kappa r^{-1 / 2}$ and so the fixed point, $b$, given by Lemma 3.5 has norm $\|\mathfrak{b}\|_{\mathbb{H}} \leq \kappa r^{-1 / 2}$.

Proof of Lemma 3.6 The part of $\mathfrak{v}$ that comes from $r^{-1 / 2}\left(i * d \mu+\frac{1}{2} B_{A_{K}}\right)$ has $L^{2}$ norm bounded by $c_{0} r^{-1 / 2}$. Lemma 3.4 with (2-1), (2-2) and (2-22) guarantee a pointwise bound by $c_{1} e^{-\sqrt{r}|z| / 2}$ on the contribution to $\mathfrak{v}$ from both $B_{A_{\gamma}}-r\left(\psi_{\gamma}^{\dagger} \tau \psi_{\gamma}-i a\right)$ and $D_{A_{\gamma}} \psi_{\gamma}$. Here, $c_{1}$ depends only on the set $\left\{\mathfrak{c}_{\gamma}\right\}_{(\gamma, m) \in \Theta}$. Therefore, the $L^{2}$ norms of these contributions are bounded by $c_{2} r^{-1 / 2}$ where $c_{2}$ depends only on the set

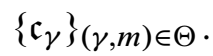

The next lemma considers how Lemma 3.6's fixed point varies with a variation in the choice of the sections $\left\{\zeta_{\gamma} \in L_{1}^{2}\left(S^{1} ; \mathfrak{c}_{\gamma}^{*} T_{1,0} \mathfrak{C}_{m}\right\}(\gamma, m) \in \Theta\right.$.

Lemma 3.7 The constant $\kappa$ in Lemma 3.6 can be chosen so that the following is true: The assignment to a data set $\left\{\zeta_{\gamma}\right\}_{(\gamma, m) \in \Theta} \in \times_{(\gamma, m) \in \Theta} L_{1}^{2}\left(S^{1} ; \mathfrak{c}_{\gamma}^{*} T_{1,0} \mathfrak{C}_{m}\right)$ of Lemma 3.6's solution of (3-16) varies smoothly in $\mathbb{H}$ as $\left\{\zeta_{\gamma}\right\}_{(\gamma, m) \in \Theta}$ varies subject to (3-1). Moreover, the directional derivative of the solution in the direction of any unit length vector in any given $(\gamma, m) \in \Theta$ version of the space $L_{1}^{2}\left(S^{1} ; \mathfrak{c}_{\gamma}^{*} T^{1,0} \mathfrak{C}_{m}\right)$ has $\mathbb{H}$ norm bounded by $\kappa r^{-1 / 2}$.

Proof of Lemma 3.7 The smooth variation of the solution with varying input is a standard consequence of the contraction mapping construction and the smooth dependence of everything on the data set $\left\{\zeta_{\gamma}\right\}$. This can be seen using (2-11), (2-12) and the constructions in Section 3.a. This understood, the issue is that of a bound on the derivative. What with (2-11) and (2-12), these bounds are obtained with arguments much like those used to prove Lemma 3.5 and Lemma 3.6. The details are straightforward and are left to the reader.

What follows is a parenthetical remark with regards to Proposition 3.1. The arguments given by Lemma 3.5 and Lemma 3.6 are, of course, much simpler for the equation

$$
\mathfrak{L}_{I} \mathfrak{b}_{0}+r^{1 / 2} \mathfrak{b}_{0} * \mathfrak{b}_{0}=\mathfrak{v},
$$

where $\mathfrak{v}$ now contains just the $-r^{-1 / 2}\left(i * d \mu+\frac{1}{2} B_{A_{K}}\right)$ term on the right side of (3-4). These arguments find a unique, small normed solution, this denoted by $\mathfrak{b}_{0}$. This 
solution has $\mathbb{H}$ norm $\left\|\mathfrak{b}_{0}\right\|_{\mathbb{H}} \leq c_{0} r^{-1 / 2}$; as explained in Lemma 3.10, it also obeys $\left|\mathfrak{b}_{0}\right| \leq c_{0} r^{-1}$. This solution to (3-34) gives the gauge equivalence class that is alluded to by Proposition 3.1.

\section{3.e Solving (3-6)}

Let $\varepsilon_{0}$ denote the constant that appears in (3-1). Fix $\varepsilon_{1}>0$ but much less than $\varepsilon_{0}$. Some additional upper bounds for $\varepsilon_{1}$ are described in what follows. With $\varepsilon_{1}$ chosen, introduce $\mathcal{B} \subset \bigoplus_{(\gamma, m) \in \Theta} L_{1}^{2}\left(S^{1} ; \mathfrak{c}_{\gamma}^{*} T^{1,0} \mathfrak{C}_{m}\right)$ denote the ball of radius $\varepsilon_{1}$. Here is the first constraint on $\varepsilon_{1}$ : Any given $\zeta \in \mathcal{B}$ has pointwise norm bounded $\varepsilon_{0}$. This understood, Lemmas 3.5-3.7 describe a smooth map $\mathfrak{b}: \mathcal{B} \rightarrow H$ such that $\mathfrak{b}(\zeta) \in \mathbb{H}_{\zeta} \frac{\perp}{\zeta}$ solves $\zeta$ 's version of (3-16). With $\zeta=\left(\zeta_{\gamma} \in L_{1}^{2}\left(S^{1} ; \mathfrak{c}_{\gamma}^{*} T_{1,0} \mathfrak{C}_{m}\right)\right)_{(\gamma, m) \in \Theta} \in \mathcal{B}$ chosen, then $\mathfrak{b}(\zeta)$ is a solution to (3-6) if and only if

$$
\Pi_{\gamma \zeta} \cdot\left(\mathfrak{L}_{\mathfrak{c} \Theta \mathfrak{J}} \mathfrak{b}+r^{1 / 2} \mathfrak{b} * \mathfrak{b}-\mathfrak{v}\right)=0
$$

for each $(\gamma, m) \in \Theta$. The task now is to find $\varepsilon_{1}$ so that (3-35) holds for one and only one choice of $\zeta \in \mathcal{B}$. The pair depicted by (3-4) solves (1-13) when (3-35) holds.

The first step in this task is to rewrite (3-35) in a more suggestive fashion. To this end, note that the subspace $\mathbb{L}_{\gamma \zeta} \in \mathbb{L}$ is canonically isomorphic to the space of $L^{2}$ sections over $S^{1}$ of $\mathfrak{c}_{\gamma, \zeta^{*}} T_{1,0} \mathfrak{C}_{m}$. This isomorphism arises as follows: At any given $t \in S^{1}$, the kernel of the $\mathfrak{c}=\mathfrak{c}_{\gamma, \zeta}(t)$ version of the operator $\vartheta_{\mathfrak{c}}$ is isomorphic to $T_{1,0} \mathfrak{C}_{m}$ at $\mathfrak{c}_{\gamma, \zeta}(t)$. Meanwhile, the operator $\left.\hat{\vartheta}_{\gamma, r}\right|_{t}$ is obtained from this same version of $\vartheta_{\mathfrak{c}}$ by rescaling $\mathbb{C}$ by the factor $r_{\gamma}^{1 / 2}$. In particular, the kernel of the one is obtained from the kernel of the other by rescaling. Introduce the function $\chi_{*}$ on $\mathbb{C}$ given by the rule $\chi_{*}(z)=\chi\left(|z| / 2 \rho_{*}\right)$. Multiplying the kernel of $\left.\hat{\vartheta}_{\gamma, r}\right|_{t}$ by the factor $\chi_{*}$ defines a linear injection from the kernel into $C^{\infty}\left(\mathbb{C} ; \mathbb{V}_{0}\right)$. Meanwhile, the differential at $\zeta_{\gamma}$ of the map exp $\operatorname{ex}_{\mathfrak{c}} \gamma$ from Part 8 of Section 2.a identifies $\mathfrak{c}_{\gamma, \zeta^{*}} T_{1,0} \mathfrak{C}_{m}$ with $\mathfrak{c}_{\gamma}^{*} T_{1,0} \mathfrak{C}_{m}$. Granted these identifications, then (3-35) defines a smooth map

$$
\mathcal{F}: \mathcal{B} \rightarrow \bigoplus_{(\gamma, m) \in \Theta} L^{2}\left(S^{1} ; \mathfrak{c}_{\gamma}^{*} T^{1,0} \mathfrak{C}_{m}\right) .
$$

The assigned task is to find $\varepsilon_{1}$ so that $\mathcal{F}$ has a unique zero in the radius $\varepsilon_{1}$ version of $\mathcal{B}$.

Lemma 3.8 Let $\Theta$ be as in (3-1), but now choose the data $\left\{\mathfrak{c}_{\gamma}: S^{1} \rightarrow \mathfrak{C}_{m}\right\}_{(\gamma, m)}$ so that each $\mathfrak{c}_{\gamma}$ is a nondegenerate solution to (1-10). Fix $\sigma \in(0,1 / 2)$. Then there exists $\kappa \geq 1$ with the following significance: Take $r \geq \kappa, \varepsilon_{1}=\kappa^{-1}$ and $\rho_{*}$ obeying $r^{-\sigma} \leq \rho_{*} \leq \kappa^{-1}$. There is a unique solution in $\mathcal{B}$ to the equation $\mathcal{F}=0$. This solution has $L_{1}^{2}$ norm bounded by $\kappa r^{-1 / 4}$. 
Here is a parenthetical remark: The key estimate in the proof of Lemma 3.8 fails when the assumption about (1-10) is dropped. Indeed, (1-10) enters the SeibergWitten/embedded contact homology story solely for its use in Lemma 3.8.

Proof of Lemma 3.8 The proof of this lemma also uses perturbation theoretic constructions. The proof has five steps. The first four focus attention on a given $(\gamma, m) \in \Theta$ component of $\mathcal{F}$. Denote the latter by $\mathcal{F}_{\gamma}$.

Step 1 The section $\mathcal{F}_{\gamma}$ of $\mathfrak{c}_{\gamma}^{*} T_{1,0} \mathfrak{C}_{m}$ is defined by first rescaling via $r_{\gamma}^{1 / 2}$ a certain $L^{2}$ map from $S^{1} \times \mathbb{C}$ to $\mathbb{V}_{0} \oplus \mathbb{V}_{1}$, then projecting to the kernel of $\vartheta_{c_{\gamma, \zeta(\cdot)}}$, and then using the inverse of the differential of the map $\exp _{\mathfrak{c}_{\gamma}}$ at $\zeta_{\gamma}$. This operation can be performed on any given $L^{2}$ map from $S^{1} \times \mathbb{C}$ to $\mathbb{V}_{0} \oplus \mathbb{V}_{1}$. Let $\mathfrak{f}=\left(\mathfrak{f}_{0}, \mathfrak{f}_{1}\right)$ denote such a map. What follows describes the resulting section of $\mathfrak{c}_{\gamma}^{*} T_{1,0} \mathfrak{C}_{m}$.

Fix a trivializing, orthonormal basis $\left\{\theta_{j 0}\right\}_{1 \leq j \leq m}$ for $\mathfrak{c}_{\gamma}^{*} T_{1,0} \mathfrak{C}_{m}$. At any given $t \in S^{1}$, the differential of $\exp _{\mathfrak{c}_{\gamma}(t)}$ at $\left.\zeta_{\gamma}\right|_{t}$ identifies $\left\{\left.\theta_{j 0}\right|_{t}\right\}_{1 \leq j \leq m}$ with a basis $\left\{\theta_{j}\right\}_{1 \leq j \leq m}$ for the kernel the operator $\vartheta_{c_{\gamma, \xi(t)}}$. Each $\theta_{j}$ is a map from $\mathbb{C}$ to $\mathbb{C} \oplus \mathbb{C}$ that obeys the $\left(A^{\zeta}, \alpha^{\zeta}\right)$ version of (1-7). The map $\mathfrak{f}$ defines the section of $\mathfrak{c}_{\gamma}^{*} T_{1,0} \mathfrak{C}_{m}$ whose value at $t \in S^{1}$ has the form

$$
\sum_{1 \leq j \leq n} \theta_{j 0} r_{\gamma} \frac{1}{\pi} \int_{\mathbb{C}}\left(\left(\hat{r}_{\gamma}^{*} \theta_{j}\right)^{\dagger} \mathfrak{f}_{0}\right) \chi_{*}(1+\mathfrak{e}) d^{2} z
$$

where $\left(\hat{r}_{\gamma}^{*} \theta_{j}\right)(z)=\theta_{j}\left(r_{\gamma}^{1 / 2} z\right)$ and where $|\mathfrak{e}| \leq c_{0}|z|^{2}$. Here, $\chi_{*}(z)=\chi\left(|z| / \rho_{*}\right)$. The section $\mathcal{F}_{\gamma}$ is defined by (3-37) with $\mathfrak{f}$ given by the expression that appears in the brackets on the left side of (3-35).

Step 2 Do not assume yet that the map $\mathfrak{c}_{\gamma}$ is a solution to (1-10). Working from left to right, consider the size of the various terms from (3-35) that make up the map $\mathcal{F}_{\gamma}$. The term $\Pi_{\gamma \zeta}\left(\mathfrak{L}_{\mathfrak{c}_{\Theta \mathfrak{J}}} \mathfrak{b}\right)$ involves solely the $\mathbb{V}_{0}$ component of $\mathfrak{L}_{\mathfrak{c}_{\Theta \mathfrak{J}}}$, this given by (3-13). To bound this contribution to $\mathcal{F}_{\gamma}$, write $\mathfrak{b}$ as a pair $\left(\lambda_{0}, \lambda_{1}\right)$ with $\lambda_{0}$ and $\lambda_{1}$ respective maps to $\mathbb{V}_{0}$ and $\mathbb{V}_{1}$. The component $\lambda_{1}$ appears in (3-13) as $\hat{\vartheta}_{\gamma, r}^{\dagger} \lambda_{1}$ plus terms with norm bounded by $c_{\mathrm{c}}\left(|z|\left|\nabla \lambda_{1}\right|+\left|\lambda_{1}\right|\right)$. Here, $\nabla$ is the covariant derivative defined by $A^{\gamma}$. Meanwhile, $\hat{\vartheta}_{\gamma, r}^{\dagger} \lambda_{1}$ is orthogonal on $\mathbb{C}$ to $\hat{r}_{\gamma}^{*} \theta_{j}$ as can be seen with an integration by parts. Thus, (3-37) with Hölder's inequality and (2-2) imply that the component $\lambda_{1}$ contributes at most $c_{0}\left\|\mathfrak{b}_{\gamma}\right\|_{\mathbb{H}}$ to the $L^{2}$ norm of $\mathcal{F}_{\gamma}$; and this is at most $c_{0} r^{-1 / 2}$ since $\left\|\mathfrak{b}_{\gamma}\right\|_{\mathbb{H}} \leq c_{c} r^{-1 / 2}$. The component $\lambda_{0}=\left(q, \eta_{0}\right)$ of $\mathfrak{b}$ contributes to $\Pi_{\gamma \zeta}\left(\mathfrak{L}_{\mathfrak{c}_{\Theta \mathfrak{J}}} \mathfrak{b}_{\gamma}\right)$ as $\nabla_{t, \zeta}\left(q, \eta_{0}\right)=\left((\partial / \partial t) q,\left(\partial / \partial t+A_{0}\right) \eta_{0}\right)$ plus terms that are bounded by $c_{0}\left(|z|\left|\nabla \lambda_{0}\right|+\left|\lambda_{0}\right|\right)$. As just noted, terms of the latter sort contribute at most $c_{0} r^{-1 / 2}$ to the $L^{2}$ norm of $\mathcal{F}_{\gamma}$. Meanwhile, the former contributes at most $c_{0}\left(\left\|f_{\zeta_{\gamma}}\right\|_{2} r^{-1 / 4}+r^{-1 / 2}\right)$, this a consequence of Lemma 2.5. 
It follows from (3-37) and (2-2) that the term $r^{1 / 2} \mathfrak{b} * \mathfrak{b}$ contributes at most $r^{3 / 4}\|\mathfrak{b}\|_{\mathbb{H}}^{2}$ to the $L^{2}$ norm of $\mathcal{F}_{\gamma}$, and is this bounded by $c_{0} r^{-1 / 4}$.

The term designated by $\Pi_{\gamma \zeta}(\mathfrak{v})$ in (3-35) has one contribution that comes from the term $r^{-1 / 2}\left(i * d \mu+\frac{1}{2} B_{A_{K}}\right)$ in (3-5). Given (2-2), it follows that the size of the latter's contribution to the $L^{2}$ norm of $\mathcal{F}_{\gamma}$ is bounded by $c_{0} r^{-1 / 2}$. The remainder of $\Pi_{\gamma \zeta}\left(\mathfrak{v}_{\gamma}\right)$ is considered in the next step.

Step 3 The portion of $\Pi_{\gamma \zeta}(\mathfrak{v})$ left to consider comes from $B_{A_{\gamma}}-r\left(\psi_{\gamma}^{\dagger} \tau \psi_{\gamma}-i a\right)$ and $D_{A_{\gamma}} \psi_{\gamma}$. As is explained next, their contributions to $\mathcal{F}_{\gamma}$ is the projection to $\operatorname{kernel}\left(\vartheta_{\mathfrak{c}_{\gamma}}\right)$ of what can be written as

$$
\frac{i}{2} \mathfrak{c}_{\gamma, \zeta}^{\prime}+\left.\nabla^{1,0} \hat{h}\right|_{\mathfrak{c}_{\gamma, \zeta}}+\mathfrak{e}
$$

where $|\mathfrak{e}| \leq c_{0} r^{-1 / 2}$. Here, $h$ is the function that is depicted in (1-9).

To see how (3-38) arises, use Lemma 3.4 to see that the contribution to the section $\mathcal{F}_{\gamma}$ from $B_{A_{\gamma}}-r\left(\psi_{\gamma}^{\dagger} \tau \psi_{\gamma}-i a\right)$ and $D_{A_{\gamma}} \psi_{\gamma}$ can be written as $(i / 2) c_{\gamma, \zeta}^{\prime}-\left.\mathfrak{v}\right|_{\mathfrak{c}_{\gamma, \zeta}}+\mathfrak{e}$, where $|\mathfrak{e}| \leq c_{0} r^{-1 / 2}$ and where the inner product between $\mathfrak{v}$ and any given $\eta=$ $(q, \varsigma) \in \vartheta_{\mathfrak{c}_{\gamma}}$ is

$$
\langle\eta, \mathfrak{v}\rangle=\frac{1}{\pi} \int_{\mathbb{C}}(v z+\mu \bar{z})\left(\bar{\zeta} \partial_{A} \alpha+\frac{1}{\sqrt{2}} \bar{q}\left(1-|\alpha|^{2}\right)\right) .
$$

What with (2-13), this implies that $\mathfrak{v}=\nabla^{1,0} \mathfrak{h}$.

Step 4 Given what is said in the preceding steps, it follows that $\mathcal{F}_{\gamma}$ can be written now as

$$
\mathcal{F}_{\gamma}=\frac{i}{2} \mathfrak{c}_{\gamma}^{\prime}+\left.\nabla^{1,0} \mathfrak{h}\right|_{\mathfrak{c}_{\gamma}}+\frac{i}{2} \nabla_{t} \zeta_{\gamma}+\left.\left(\nabla_{\zeta_{\gamma}} \nabla^{1,0} h\right)\right|_{\mathfrak{c}_{\gamma}}+\mathfrak{R}_{\gamma}
$$

where $\mathfrak{R}_{\gamma}$ is an $L^{2}$ section of $\mathfrak{c}_{\gamma}^{*} T_{1,0} \mathfrak{C}_{m}$ that obeys (3-40)

$$
\left\|\Re_{\gamma}\right\|_{2} \leq c_{\mathfrak{c}}\left(r^{-1 / 4}+\left\|\left.\nabla_{t} \zeta_{\gamma}\right|_{2} ^{2}+\right\| \zeta_{\gamma} \|_{2}^{2}\right) .
$$

Here, the $\nabla_{t}$ is the covariant derivative on $L_{1}^{2}\left(S^{1} ; \mathfrak{c}_{\gamma}^{*} T_{1,0} \mathfrak{C}_{m}\right)$ that is defined by the pullback via $\mathfrak{c}_{\gamma}$ of the Levi-Civita connection on $T_{1,0} \mathfrak{C}_{m}$. The constant $c_{\mathfrak{c}}$ is independent of $r$ and $\zeta$, though not of $\mathfrak{c}_{\gamma}$.

The small size of $\mathfrak{R}_{\gamma}$ suggests a perturbative approach to finding $\zeta \in \mathcal{B}$ that makes each $(\gamma, m) \in \Theta$ version of $\mathcal{F}_{\gamma}$ equal to zero. This requires, first of all, that $\mathfrak{c}_{\gamma}$ obey (1-10) so that the $\zeta$-independent term $\frac{i}{2} \mathfrak{c}_{\gamma}^{\prime}+\left.\nabla^{1,0} h_{\mid}\right|_{\mathfrak{c}_{\gamma}}$ contributes zero to (3-40). The most straightforward sort of perturbation theory also requires that $\mathfrak{c}_{\gamma}$ define a nondegenerate 
solution to (1-10). This says neither more nor less than the following: The operator, $L_{\gamma}$, on $C^{\infty}\left(S^{1} ; \mathfrak{c}_{\gamma}^{*} T_{1,0} \mathfrak{C}_{m}\right)$ that sends a section $\eta$ to $L_{\gamma} \eta=(i / 2) \nabla_{t} \eta+\left.\left(\nabla_{\eta} \nabla^{1,0} h\right)\right|_{\mathfrak{c}_{\gamma}}$ has trivial kernel. The operator $L_{\gamma}$ has a bounded inverse mapping $L^{2}\left(S^{1} ; \mathfrak{c}_{\gamma}^{*} T_{1,0} \mathfrak{C}_{m}\right)$ to $L_{1}^{2}\left(S^{1} ; \mathfrak{c}_{\gamma}^{*} T_{1,0} \mathfrak{C}_{m}\right)$ when its kernel is trivial.

Step 5 Assume now that each element in the set $\left\{\mathfrak{c}_{\gamma}\right\}_{(\gamma, m) \in \Theta}$ is a nondegenerate solution to (1-10). Define a map $\mathbb{P}: \mathcal{B} \rightarrow \bigoplus_{(\gamma, m) \in \Theta} L_{1}^{2}\left(S^{1} ; \mathfrak{c}_{\gamma}^{*} T_{1,0} \mathfrak{C}_{m}\right)$ by setting its component in a given $(\gamma, m)$ summand to be $\mathbb{P}_{\gamma}=-L_{\gamma}^{-1} \mathfrak{R}_{\gamma}$. A point $\zeta \in \mathcal{B}$ is a solution to the equation $\mathcal{F}=0$ if and only if it is a fixed point of $\mathbb{P}$.

Lemma 3.9 Fix $\sigma \in(0,1 / 2)$. Given that each element in the set $\left\{\mathfrak{c}_{\gamma}\right\}_{(\gamma, m) \in \Theta}$ is a nondegenerate solution to (1-10), there exists a constant $\kappa \geq 1$ such that if $r \geq \kappa, \varepsilon_{1}=$ $\kappa^{-1}$, and $r^{-\sigma} \leq \rho_{*} \leq \kappa^{-1}$, then the following is true: The map $\mathbb{P}$ sends $\mathcal{B}$ to itself as a contraction mapping. Thus, it has a unique fixed point in $\mathcal{B}$. Moreover, any given $(\gamma, m) \in \Theta$ component of this fixed point is a smooth section over $S^{1}$ of $\mathfrak{c}_{\gamma}^{*} T_{1,0} \mathfrak{C}_{m}$ with $L_{1}^{2}$ norm bounded by $\kappa r^{-1 / 4}$.

Proof of Lemma 3.9 Since $L_{\gamma}$ is invertible, it follows that the $L_{1}^{2}$ norm of $\mathbb{P}_{\gamma}$ is bounded by an $r$ and $\zeta$ independent constant times $\left\|\Re_{\gamma}\right\|_{2}$. Thus, (3-41) guarantees a bound by $c_{0}\left(\varepsilon_{1}^{2}+r^{-1 / 4}\right)$ on the $L_{1}^{2}$ norm of $\mathbb{P}_{\gamma}$. As a consequence, there exists $c_{0}>1$ such that $\mathbb{P}$ maps $\mathcal{B}$ to itself if $r \geq c_{0}$ and $\varepsilon_{1} \leq 1 / c_{0}$.

It remains yet to prove that $\kappa$ can be chosen so as to guarantee that $\mathbb{P}$ is a contraction mapping on $\mathcal{B}$. This follows with a proof that

$$
\left\|\Re_{\gamma}(\zeta)-\Re_{\gamma}\left(\zeta^{\prime}\right)\right\|_{2} \leq c_{0}\left(\|\zeta\|_{L_{1}^{2}}+\left\|\zeta^{\prime}\right\|_{L_{1}^{2}}+r^{-1 / 4}\right)\left\|\zeta-\zeta^{\prime}\right\|_{L_{1}^{2}}
$$

The existence of such a bound follows from the bounds given in (2-11), (2-12) and Lemma 3.7 after differentiating the various terms that appear in (3-35). The derivation of (3-42) from these bounds is straightforward; it involves arguments that are, but for minor cosmetic changes, the same as those used in the proof of Lemma 3.4 and Steps 1-4 in this subsection. This said, the proof of (3-42) is left to the reader.

Given that $\mathbb{P}$ is a contraction mapping, the bound by $c_{0} r^{-1 / 4}$ on the $L_{1}^{2}$ norm of its fixed point in $\mathcal{B}$ follows from the bound in (3-41). The fact that any given $(\gamma, m) \in \Theta$ component of this fixed point is smooth can be established using standard boot strapping techniques for first order differential equations. This is can be done in an iterative fashion by alternately using (3-5) to incrementally increase the differentiability of $\mathfrak{b}$ via techniques from [3, Chapter 6] and then using the equations $\left\{\mathcal{F}_{\gamma}\right\}_{(\gamma, m) \in \Theta}=0$ with $\mathcal{F}_{\gamma}$ as in (3-40) to increase the differentiability of $\left\{\zeta_{\gamma}\right\}_{(\gamma, m) \in \Theta}$ using the fact that the latter can be viewed as an elliptic system of first order, ordinary differential equations. 
There is a somewhat more direct way to see why the pair $(A, \psi)$ given in (3-4) is smooth. To elaborate, write $(A, \psi)$ as $\left(A^{*}+\hat{a}, \psi^{*}+\lambda\right)$ where $\left(A^{*}, \psi^{*}\right)$ is the version of $\left(A_{\mathfrak{J}}, \psi_{\mathfrak{J}}\right)$ that is defined by taking $\mathfrak{J}=\left\{\left(\mathfrak{c}_{\gamma}, \zeta_{\gamma}=0\right)\right\}_{(\gamma, m) \in \Theta}$. The pair $\left(A^{*}, \psi^{*}\right)$ is smooth. Let $\phi$ denote the $i \mathbb{R}$ component of $\mathfrak{b}$. What with (2-9), (2-10) and (3-4), it follows that the triple $(\hat{a}, \lambda, \phi)$ obeys an elliptic, first order, nonlinear equation with quadratic nonlinearities. Apriori, this triple lies in the Sobolev space $L_{1}^{2}\left(M ; i T^{*} M \oplus \mathbb{S} \oplus i \mathbb{R}\right)$. Elliptic bootstrapping techniques (see, for example, Morrey $[3$, Chapter 6$])$ can be used to prove directly that $(\hat{a}, \lambda, \phi)$ are smooth. Of course, it is known that any $L_{1}^{2}$ solution of (1-13) is apriori gauge equivalent to a smooth solution (see, eg Kronheimer and Mrowka [2].)

\section{3.f Other properties of the solution}

The next lemma says more about the zero $\zeta \in \mathcal{B}$ of $\mathcal{F}$ and the corresponding point $\mathfrak{b}(\zeta)$. It is used in a subsequent section.

Lemma 3.10 There exists a constant $\kappa>1$ with the following significance: Fix $r \geq \kappa$ and let $\zeta \in \mathcal{B}$ denote the zero of $\mathcal{F}$ that is provided by Lemma 3.8. Then the $L_{1}^{2}$ norm of $\zeta$ is bounded by $\kappa r^{-1 / 2}$. Meanwhile, let $\mathfrak{b}(\zeta) \in \mathbb{H} \frac{\perp}{\zeta}$ denote the solution to the corresponding version of (3-16) that is provided by Lemma 3.6. Then $\mathfrak{b}(\zeta)=\mathfrak{b}_{* \zeta}+\mathfrak{e} \zeta$ where

- $\left|\mathfrak{b}_{* \zeta}\right| \leq \kappa\left(r^{-1}+r^{-1 / 2} \sum_{(\gamma, m) \in \Theta} e^{-\sqrt{r} \operatorname{dist}(\cdot, \gamma) / \kappa}\right)$.

- $\left\|\mathfrak{e}_{\zeta}\right\|_{\mathbb{H}} \leq \kappa r^{-1}$.

- $|\mathfrak{b}(\zeta)| \leq \kappa r^{-1 / 2}$.

- The small solution $\mathfrak{b}_{0}$ to (3-34) obeys $\left|\mathfrak{b}_{0}\right| \leq c_{0} r^{-1}$.

Proof of Lemma 3.10 The proof has six steps. Steps 1-4 allow $\zeta$ to denote any given element in $\mathcal{B}$.

Step 1 Fix $(\gamma, m) \in \Theta$. According to Lemma 3.4,the $\zeta$ version of $\mathfrak{v}$ on $U_{\gamma}$ can be viewed as a map to the vector space $\mathbb{V}_{0} \oplus \mathbb{V}_{1}$ that can be written as $\mathfrak{v}_{\gamma \zeta}+\mathfrak{z}^{\prime}$, where $\left|\mathfrak{z}^{\prime}\right| \leq c_{0} r^{-1 / 2} e^{-\sqrt{r}|z| / 2}$ and where $\mathfrak{v}_{\gamma, \zeta}$ maps to $\mathbb{V}_{0}$ and is given by

$$
\mathfrak{v}_{\gamma \zeta}=\left(x_{\zeta}^{r}-r_{\gamma}^{1 / 2}(v z+\mu \bar{z}) 2^{-1 / 2}\left(1-\left|\alpha^{\zeta, r}\right|^{2}\right), \iota_{\zeta}^{r}-(v z+\mu \bar{z}) \partial_{A^{\zeta, r}} \alpha^{\zeta, r}\right) .
$$

Note that $\mathfrak{v}_{\gamma, \zeta}$ is defined over the whole of $S^{1} \times \mathbb{C}$. Granted the preceding, let $\Pi^{0}$ denote the $L^{2}$-orthogonal projection on $\mathbb{C}$ to the kernel of (3-15) at any given $t \in S^{1}$. The latter operator is denoted by $\hat{\vartheta}_{\gamma, r}$. Let $\mathfrak{p}_{\gamma \zeta}$ denote the $L^{2}$ map from $S^{1} \times \mathbb{C}$ to $\mathbb{V}_{1}$ 
that obeys the equation $\left(2 \pi / \ell_{\gamma}\right)^{1 / 2} \hat{\vartheta}_{\gamma, r}^{\dagger} \mathfrak{p}_{\gamma \zeta}=\left(1-\Pi^{0}\right) \mathfrak{v}_{\gamma \zeta}=0$ at each $t \in S^{1}$. The bounds in (2-1), (2-2) and the formula in (2-7) can be used to prove that

$$
\left|\mathfrak{p}_{\gamma \zeta}\right|+\left|\nabla_{t \gamma} \mathfrak{p}_{\gamma \zeta}\right|+r^{-1 / 2}\left|\nabla_{A \zeta, r} \mathfrak{p}_{\gamma \zeta}\right| \leq c_{0} r^{-1 / 2} e^{-\sqrt{r}|z| / 2}
$$

Define $\mathfrak{p}_{\gamma \zeta}$ as above and view $\mathfrak{b}_{\gamma \zeta}=\left(0, \mathfrak{p}_{\gamma \zeta}\right): S^{1} \times \mathbb{C} \rightarrow \mathbb{V}_{0} \oplus \mathbb{V}_{1}$ over the $U_{\gamma}$ part of $S^{1} \times \mathbb{C}$ as a section of the vector bundle $i T^{*} M \oplus \mathbb{S} \oplus i \mathbb{R}$.

Step 2 Introduce the operator $\mathfrak{L}_{I}$ as in (3-10). Let $\mathfrak{b}_{0}$ denote the small normed solution to (3-34); this the solution with $\left\|\mathfrak{b}_{0}\right\|_{\mathbb{H}} \leq c_{0} r^{-1 / 2}$ that is provided by the analog of Lemma 3.5 and Lemma 3.6 when $\Theta=\varnothing$ (see [7, Proposition 2.8]). This $\mathfrak{b}_{0}$ obeys the equation $\mathfrak{L}_{I}^{2} \mathfrak{b}_{0}=-r^{1 / 2} \mathfrak{L}_{I}\left(\mathfrak{b}_{0} * \mathfrak{b}_{0}\right)+\mathfrak{L}_{I} \mathfrak{v}$. The operator $\mathfrak{L}_{I}^{2}$ is depicted in $[6,(5.22)]$. Given the latter, it follows that $\left|\mathfrak{b}_{0}\right|$ obeys the differential inequality

$$
d^{\dagger} d\left|\mathfrak{b}_{0}\right|+2 r\left|\mathfrak{b}_{0}\right| \leq c_{0} r\left|\mathfrak{b}_{0}\right|^{2}+c_{0} .
$$

Fix $p \in M$ and let $g(\cdot, p)$ denote the Green's function for $d^{\dagger} d+2 r$ with pole at $p$. The latter is a nonnegative function on $M-p$ and it obeys $|g(\cdot, p)| \leq$ $c_{0} \operatorname{dist}(\cdot, p)^{-1} e^{-\sqrt{r} \operatorname{dist}(\cdot, p) / c_{0}}$. In particular, its $L^{1}$ norm is bounded by $c_{0} r^{-1}$ and its $L^{2}$ norm is bounded by $c_{0} r^{-1 / 2}$. Note also that $g(x, p)=g(p, x)$. Fix $x \in M$ where $\left|\mathfrak{b}_{0}\right|$ is maximal, multiply both sides of (3-45) by $g(x, \cdot)$, and then integrate over $M$. The resulting equation implies that

$$
\left\|\mathfrak{b}_{0}\right\|_{\infty} \leq c_{0} r\left\|\mathfrak{b}_{0}\right\|_{\infty}\left\|\mathfrak{b}_{0}\right\|_{2}\|g(x, \cdot)\|_{2}+c_{0}\|g(x, \cdot)\|_{1} .
$$

Given what was said just now about the $L^{2}$ and $L^{1}$ norms of $g(\cdot, p)$, the right hand side of (3-49) is bounded by $c_{0}\left(\left\|\mathfrak{b}_{0}\right\|_{\mathbb{H}}\left\|\mathfrak{b}_{0}\right\|_{\infty}+r^{-1}\right)$. Since $\left\|\mathfrak{b}_{0}\right\|_{\mathbb{H}} \leq c_{0} r^{-1 / 2}$, this last fact implies that $\left\|\mathfrak{b}_{0}\right\|_{\infty} \leq c_{0} r^{-1}$.

Step 3 Fix $R \geq 1$ in the following way: For each $(\gamma, m) \in \Theta$, view the map $\mathfrak{c}_{\gamma}$ as a map from $S^{1}$ into $\operatorname{Sym}^{m}(\mathbb{C})$. Let $R_{\gamma}$ denote the maximum as $t$ varies in $S^{1}$ of the norms of the points in $\mathbb{C}$ that define $\mathfrak{c}_{\gamma}$. Set $R=\sum_{(\gamma, m) \in \Theta} R_{\gamma}+100$. Let $\chi_{\gamma^{*}}$ denote the function on $C$ given by $z \rightarrow \chi\left(r_{\gamma}^{1 / 2}|z| / R\right)$. View this as a function on $M$ with support on $U_{\gamma}$. Set $\chi_{0 *}$ to denote the function $1-\sum_{(\gamma, m)} \chi_{\gamma *}$.

By virtue of the choice for $R$, the identifications in Steps 1 and 2 of Section 3.a that define $E$ over each $U_{\gamma}$ and $E$ over $U_{0}$ extend to identify $E$ as the trivial bundle over the support of $\chi_{0}$. Thus they identify $i T^{*} M \oplus \mathbb{S} \oplus i \mathbb{R}$ with the bundle $i T^{*} M \oplus \mathbb{S}_{I} \oplus i \mathbb{R}$ over the support of $\chi_{0}$. With these identifications understood, then $\chi_{0 *} \mathfrak{b}_{0}^{0}$ defines a section of $i T^{*} M \oplus \mathbb{S} \oplus i \mathbb{R}$ over the whole of $M$. Meanwhile, each $(\gamma, m) \in \Theta$ version of $\chi_{\gamma} \mathfrak{b}_{\gamma \zeta}$ also defines a section of $i T^{*} M \oplus \mathbb{S} \oplus i \mathbb{R}$ over the whole of $M$. 
Granted these identifications, set

$$
\mathfrak{b}_{* \zeta}=\left(1-\Pi_{\zeta}\right)\left(\chi_{0 *} \mathfrak{b}_{0}+\sum_{(\gamma, m) \in \Theta} \chi_{\gamma} \mathfrak{b}_{\gamma \zeta}\right) .
$$

Step 4 It is a consequence of (2-1), (3-47), (3-46) and (3-13)-(3-14) with Lemma 3.4 that

$$
\left(1-\Pi_{\zeta}\right)\left(\mathfrak{L}_{\mathfrak{c}_{\Theta \mathfrak{J}}} \mathfrak{b}_{* \zeta}+r^{1 / 2} \mathfrak{b}_{* \zeta} * \mathfrak{b}_{* \zeta}-r^{-1 / 2} \mathfrak{v}\right)
$$

has $L^{2}$ norm bounded by $c_{0} r^{-1}$. Note as well that $\left\|\mathfrak{b}_{* \zeta}\right\|_{\mathbb{H}} \leq c_{0} r^{-1 / 2}$. Denote what is written in (3-48) by $\mathfrak{v}_{* \zeta}$. Write the section $\mathfrak{b} \in \mathbb{H} \frac{\perp}{\zeta}$ that is supplied by Lemma 3.6 as $\mathfrak{b}=\mathfrak{b}_{* \zeta}+\mathfrak{e} \xi$. As $\mathfrak{b}$ obeys (3-16), so $\mathfrak{e} \zeta$ must obey the equation

$$
\left(1-\Pi_{\zeta}\right)\left(\mathfrak{L}_{\mathfrak{c}_{\Theta \mathfrak{J}}} \mathfrak{e} \zeta+r^{1 / 2} \mathfrak{e}_{\zeta} * \mathfrak{e}_{\zeta}+2 r^{1 / 2} \mathfrak{b}_{* \zeta} * \mathfrak{e}_{\zeta}-\mathfrak{v}_{* \zeta}\right)=0 .
$$

As $\|\mathfrak{e} \xi\|_{\mathbb{H}} \leq\|\mathfrak{b}\|_{\mathbb{H}}+\left\|\mathfrak{b}_{* \xi}\right\|_{\mathbb{H}} \leq c_{0} r^{-1 / 2}$, (3-31) and Lemma 3.2 imply $\|\mathfrak{e} \xi\|_{\mathbb{H}} \leq c_{0} r^{-1}$.

Step 5 Take $\zeta \in \mathcal{B}$ to be the element that is supplied by Lemma 3.8. To refine the upper bound for the $L_{1}^{2}$ norms $\zeta=\left(\zeta_{\gamma}\right)_{(\gamma, m) \in \Theta}$, return to Step 2 of the proof of Lemma 3.8. The latter derived a bound of $c_{0} r^{-1 / 4}$ for the contribution to the $L^{2}$ norm of $\mathcal{F}$ from the term $\Pi_{\zeta}\left(r^{1 / 2} \mathfrak{b} * \mathfrak{b}\right)$ that appears in (3-35). As is argued next the latter contributes at most $c_{0} r^{-1 / 2}$ to the $L^{2}$ norm of $\mathcal{F}$. Granted that such is the case, it then follows that the left hand side of (3-41) can be replaced by $c_{0}\left(r^{-1 / 2}+\left\|\nabla_{t} \zeta_{\gamma}\right\|_{2}^{2}+\left\|\zeta_{\gamma}\right\|_{2}^{2}\right)$. The contraction mapping theorem used to find $\zeta$ now gives a bound of $c_{0} r^{-1 / 2}$ on its $L_{1}^{2}$ norm.

To bound the contribution of $\Pi_{\zeta}\left(r^{1 / 2} \mathfrak{b} * \mathfrak{b}\right)$ to the $L^{2}$ norm of $\mathcal{F}$, write $\mathfrak{b}=\mathfrak{b}_{* \zeta}+\mathfrak{e} \xi$. Given (3-37), it follows using Hölder's inequality that the contribution to the $L^{2}$ norm of $\mathcal{F}$ from this term is bounded by $r^{1 / 2}\left(\left|\mathfrak{b}_{* \zeta}\right|_{L^{\infty}}\right)^{2}+r^{3 / 4}\|\mathfrak{e} \xi\|_{\mathbb{H}}^{2}$. The latter is bounded by $c_{0} r^{-1 / 2}$.

Step 6 Introduce $\mathfrak{J}_{0}=\left\{\left(\mathfrak{c}_{\gamma}, 0\right)\right\}_{(\gamma, m) \in \Theta}$ and let $\mathfrak{L}_{\mathfrak{c}_{\Theta \mathfrak{J}_{0}}}$ denote the $\left(A_{\mathfrak{J}_{0}}, \psi_{\mathfrak{J}_{0}}\right)$ version of the operator that appears in (3-8). This operator differs from the operator $\mathfrak{L}_{\mathfrak{c}_{\Theta \mathfrak{J}}}$ that appears in (3-6) by zero-th order terms that are bounded pointwise by $c_{0} r^{1 / 2}|\zeta| \sum_{(\gamma, \mu) \in \Theta} e^{-\sqrt{r} \operatorname{dist}(\gamma, \cdot) / c_{0}}$. Note that the coefficients of the derivatives appearing in $\mathfrak{L}_{\mathfrak{c}_{\Theta \mathfrak{v}} 0}$ are determined by the metric and are independent of $\Theta$ and $r$. Meanwhile, on any given ball in $M$ of radius $c_{0}^{-1}$, there is a trivialization of $E$ such that the following is true: When $\mathfrak{L}_{\mathfrak{c}_{\Theta \mathfrak{J}_{0}}}$ is written with respect to this trivialization, its zero-th order coefficients are bounded by $c_{0}\left(1+r^{1 / 2} \sum_{(\gamma, \mu) \in \Theta} e^{-\sqrt{r} \operatorname{dist}(\gamma, \cdot) / c_{0}}\right)$. In addition their derivatives to a given order $k$ are bounded by $c_{k}\left(1+r^{(k+1) / 2} \sum_{(\gamma, m) \in \Theta} e^{-\sqrt{r} \operatorname{dist}(\gamma, \cdot) / c_{0}}\right.$. 
Granted these last remarks, it then follows using a standard parametrix expansion that if $\mathfrak{f}$ and $\mathfrak{w}$ are sections of $i T^{*} M \oplus \mathbb{S} \oplus i \mathbb{R}$ and $\mathfrak{L}_{\mathfrak{c}_{\Theta \mathfrak{J}} 0} \mathfrak{f}=\mathfrak{w}$, then

(3-50) $\|\mathfrak{f}\|_{\infty} \leq c_{0} \sup _{x \in M} \int_{\operatorname{dist}(x, \cdot) \leq 1 / \sqrt{r}} \frac{1}{\operatorname{dist}(x, \cdot)^{2}}|\mathfrak{f}|+c_{0} r^{3 / 2} \sup _{x \in M} \int_{\operatorname{dist}(x, \cdot) \leq 1 / \sqrt{r}}|\mathfrak{f}|$.

Step 7 This step proves the asserted bound on $|\mathfrak{b}(\zeta)|$. To this end, remark that $\mathfrak{b}=\mathfrak{b}(\zeta)$ obeys an equation of the form $\mathfrak{L}_{\mathfrak{c}_{\Theta \mathfrak{J}_{0}}} \mathfrak{b}=\mathfrak{w}$ where $|\mathfrak{w}| \leq c_{0} r^{1 / 2}\left(|\zeta||\mathfrak{b}|+|\mathfrak{b}|^{2}\right)+$ $r^{-1 / 2}|\mathfrak{v}|$. This understood, it follows that (3-53) holds with $\mathfrak{f}=\mathfrak{b}$ and with $|\mathfrak{w}|$ just described. References are to this version of (3-53) in what follows.

Write $\mathfrak{b}=\mathfrak{b}_{* \zeta}+e_{\zeta}$. The $\mathfrak{b}_{* \zeta}$ contributions from $r^{1 / 2}|\zeta \| \mathfrak{b}|$ to the left most integral on the right hand side of (3-53) is bounded by $c_{0} r^{-1 / 2}\left\|\mathfrak{b}_{* \zeta}\right\|_{\infty}$ and that from $r^{1 / 2}|\mathfrak{b}|^{2}$ is bounded by $c_{0}\left\|\mathfrak{b}_{* \zeta}\right\|_{\infty}^{2}$. The former bound uses the bound by $c_{0} r^{-1 / 2}$ on the $L_{1}^{2}$ norm of $\zeta$ from Step 5, and the consequent bound by $c_{0} r^{-1 / 2}$ on $|\zeta|$. Granted the first bullet of Lemma 3.10, it follows that the $\mathfrak{b}_{* \zeta}$ contribution to the left most integral on the right in (3-53) is bounded by $c_{0} r^{-1}$. Meanwhile, the contribution of $\mathfrak{b}_{* \zeta}$ to the right most integral on the right hand side of (3-53) is bounded by $c_{0} r^{-1 / 2}$.

To bound the contribution from $\mathfrak{e} \xi$ to the left most integral on the right in (3-50), note first that if $\mathfrak{f} \in \mathbb{H}$, then $\left\|\operatorname{dist}(x, \cdot)^{-1} \mathfrak{f}\right\|_{2}$ is bounded by $c_{0}\|\mathfrak{f}\|_{\mathbb{H}}$ using a standard Sobolev inequality. This understood, it follows that the contribution of $r^{1 / 2}|\zeta||\mathfrak{e} \zeta|$ to the left most integral on the right hand side of (3-50) is no greater than $c_{0} r^{1 / 4}\left\|\mathfrak{e}_{\xi}\right\|_{\mathbb{H}}$, and that the contribution of $r^{1 / 2}|\mathfrak{e} \xi|^{2}$ to this same integral is no greater than $r^{1 / 2}\|\mathfrak{e} \xi\|_{\mathbb{H}}^{2}$. Meanwhile, the contribution to the far right integral on the right hand side of (3-53) from $\mathfrak{e}_{\zeta}$ is no more than $r^{1 / 4}\left\|\mathfrak{e}_{\xi}\right\|_{\mathbb{H}}$. Thus, the $\mathfrak{e}_{\zeta}$ contributions are no greater than $c_{0} r^{-3 / 4}$.

To bound the contribution to the right most integral on the right hand side of (3-50) from $r^{-1 / 2}|\mathfrak{v}|$, use Step 1 to see that $r^{-1 / 2}|\mathfrak{v}| \leq c_{0}\left(r^{-1 / 2}+|\nabla \zeta|\right) \sum_{(\gamma, m) \in \Theta} \mathfrak{e}^{-\sqrt{r} \operatorname{dist}(\gamma, \cdot) / c_{0}}$. It follows as a consequence that $r^{-1 / 2}|\mathfrak{v}|$ contributes at most $c_{0}\left(r^{-1}+r^{-1 / 2} \operatorname{lnr}\|\zeta\|_{L_{1}^{2}}\right)$ to the right most integral on the right hand side of (3-50). In particular, this is less than $c_{0} r^{-3 / 4}$.

Putting all of the above together gives the bound that is claimed by the lemma.

\section{3.g Proof of Theorem 1.1}

An element in $\mathfrak{X}$ consists of the following: First, a finite set $\Theta$ whose typical element is a pair $(\gamma, m)$ with $\gamma$ a Reeb orbit and with $m$ a positive integer. As always, distinct pairs from $\Theta$ have distinct Reeb orbit components. Second, an assignment to each pair $(\gamma, m) \in \Theta$ of a nondegenerate solution $\mathfrak{c}_{\gamma}: S^{1} \rightarrow \mathfrak{C}_{m}$ of $\gamma$ 's version of (1-10). Fix $\chi \in \mathfrak{X}$. The preceding parts of this section find a $\kappa_{\chi}>1$ and then construct a solution 
to (1-13) for any given $r>\kappa_{\chi}>1$. The gauge equivalence of this solution is defined to by $\Phi^{r}(\chi)$. Given that $X$ is finite, the assignment $\chi \rightarrow \Phi^{r}(\chi)$ is defined for all $\chi \in \mathfrak{X}$ if $r \geq c_{0}$.

Consider now the claim that $\mathrm{E}<2 \pi L$ on the image of $\Phi^{r}$ when $r$ is large. For this purpose, fix $\chi \in \mathfrak{X}$. Write $\rho_{*}=r^{-1 / 2+3 \sigma}$ with $\sigma>0$. Write $\chi=(\Theta, \hat{\mathfrak{c}})$ where $\hat{\mathfrak{c}}=\left\{\mathfrak{c}_{\gamma}\right\}_{(\gamma, m) \in \Theta}$ are the solutions to (1-10). Let $\zeta=\left\{\zeta_{\gamma}\right\}_{(\gamma, m) \in \Theta}$ denote the zero of the map $\mathcal{F}$ that is provided by Lemma 3.8. Let $\mathfrak{b}(\zeta) \in \mathbb{H} \frac{\perp}{\zeta}$ denote the solution to the corresponding version of (3-16) that is provided by Lemma 3.6. Thus $\Phi^{r}$ maps $(\Theta, \hat{\mathfrak{c}})$ to the equivalence class of the pair $(A, \psi)$ that is obtained by using $\mathfrak{J}=\left\{\left(\mathfrak{c}_{\gamma}, \zeta_{\gamma}\right)\right\}(\gamma, m) \in \Theta$ and $\mathfrak{b}(\zeta)$ in (3-3). This understood, write the $i T^{*} M$ component of $\mathfrak{b}$ as $\mathfrak{b}$. Thus, $A=A_{\mathfrak{J}}+2^{1 / 2} r^{1 / 2} \mathfrak{b}$.

To continue, write

$$
B_{A}=\left(1-\sum_{(\gamma, m) \in \Theta} \chi_{\gamma}\right) B_{A}+\sum_{(\gamma, m) \in \Theta} \chi_{\gamma} B_{A} .
$$

The integral that defines $\mathrm{E}(A)$ thus has a contribution from each of the terms on the right hand side of (3-51). The contribution from the left most term on the right hand side of (3-51) can be bounded via an integration by parts by

$$
c_{0} r^{1 / 2} \int_{M}|\mathfrak{b}|\left(1+\sum_{(\gamma, m) \in \Theta}\left|d \chi_{\gamma}\right|\right)+c_{0} r^{-1}
$$

Note in this regard that $A_{\mathfrak{J}}$ is flat where all $\chi_{\gamma}$ are zero. It follows from Lemma 3.10 that what is written in (3-52) is bounded by $c_{0} r^{-3 \sigma}$.

This last estimate implies that any significant contribution to $E$ comes from the radius $\rho_{*}$ neighborhoods of the Reeb orbits that appear in $\Theta$. To see about this contribution, fix $(\gamma, m) \in \Theta$. An analogous integration by parts writes the contribution to $\mathrm{E}$ from $\chi_{\gamma} B_{A}$ as

$$
i \int_{U_{\gamma}} a \wedge * B_{A_{\gamma}}+\mathfrak{e},
$$

where $A_{\gamma}$ is defined in (3-3) and where $|\mathfrak{e}|$ is bounded by what is written in (3-51). In particular, it again follows from Lemma 3.10 that $|\mathfrak{e}| \leq c_{0} r^{-3 \sigma}$. Given (1-1)'s depiction of a on $U_{\gamma}$, it follows from (1-4) and (3-3) that the integral in (3-53) differs from $2 \pi m \ell_{\gamma}$ by a number with absolute value less than $c_{0} r^{-1 / 2}$. Given the bound on (3-52) by $c_{0} r^{-3 \sigma}$, this last conclusion implies that

$$
\mathrm{E}=2 \pi \sum_{(\gamma, m) \in \Theta} m \ell_{\gamma}+\mathfrak{e}^{\prime}
$$

where $\left|\mathfrak{e}^{\prime}\right| \leq c_{0} r^{-3 \sigma}$. This last bound implies that $\mathrm{E}<2 \pi L$ when $r \geq c_{0}$. 
Consider next the claim that $\Phi^{r}$ is injective. To start, remark that what was said in the preceding paragraphs has the following additional implication: The map $\Phi^{r}$ maps pairs $(\Theta, \hat{\mathfrak{c}})$ and $\left(\Theta^{\prime}, \hat{\mathfrak{c}}^{\prime}\right)$ to the same gauge equivalence class if and only if $\Theta=\Theta^{\prime}$.

Suppose that $(\Theta, \hat{\mathfrak{c}})$ and $\left(\Theta, \hat{\mathfrak{c}}^{\prime}\right)$ are distinct elements in $\mathfrak{C Z}^{L^{*}}$ and that $(\gamma, m) \in \Theta$ is given distinct maps from $S^{1}$ to $\mathfrak{C}_{m}$ by $\hat{\mathfrak{c}}$ and $\hat{\mathfrak{c}}$. Let $\mathfrak{c}=(A, \alpha)$ and $\mathfrak{c}^{\prime}=\left(A^{\prime}, \alpha^{\prime}\right)$ denote these two maps. Since $\mathfrak{c} \neq \mathfrak{c}^{\prime}$, there is a point $t \in S^{1}$ and a zero $z \in \mathbb{C}$ of $\left.\alpha\right|_{t}$ that is not a zero of $\left.\alpha^{\prime}\right|_{t}$. More to the point, because $\mathfrak{X}$ is a finite set, there exists a constant $T_{L}>1$ with the following two properties: First, $T_{L}$ depends only on $L$. Second, there exists a point $t \in S^{1}$ and a point $p_{*} \subset \mathbb{C}$ such that $|\alpha|<(1 / 100) T_{L}^{-1}$ and $\left|\alpha^{\prime}\right| \geq T_{L}^{-1}$ at $(t, p)$.

Keeping this in mind, let $\zeta$ and $\zeta^{\prime}$ denote the elements that are provided by Lemma 3.8 using $\hat{\mathfrak{c}}$ and $\hat{\mathfrak{c}}^{\prime}$ respectively. Let $\left(A_{\mathfrak{J}}, \psi_{\mathfrak{J}}=\left(\alpha_{\mathfrak{J}}, \beta_{\mathfrak{J}}\right)\right)$ and $\left(A_{\mathfrak{J}^{\prime}},\left(\alpha_{\mathfrak{J}^{\prime}}, \beta_{\mathfrak{J}^{\prime}}\right)\right)$ denote the corresponding versions of the data given in Step 4 of Section 3.a. It follows from what is said in Lemma 3.10 about the size of $\zeta$ and $\zeta^{\prime}$ that $\left|\alpha_{J}\right|<(1 / 64) T_{L}^{-1}$ and $\left|\alpha_{J^{\prime}}\right| \geq(1 / 2) T_{L}^{-1}$ at the point $(t, p)$ if $r \geq c_{0}$.

Write the $E \subset E \oplus E K$ summand of the spinor component of $\Phi^{r}(\Theta, \hat{\mathfrak{c}})$ as $\alpha_{\mathfrak{J}}+\eta$ and that of $\Phi^{r}\left(\Theta, \hat{\mathfrak{c}}^{\prime}\right)$ as $\alpha_{\mathfrak{J}^{\prime}},+\eta^{\prime}$. Use the bounds $|\mathfrak{b}(\zeta)| \leq c_{0} r^{-1 / 2}$ and $\left|b\left(\zeta^{\prime}\right)\right| \leq c_{0} r^{-1 / 2}$ with what was said in the previous paragraph to see that $\left|\alpha_{\mathfrak{J}}+\eta\right|$ is bounded by $(1 / 32) T_{L}^{-1}$ at $(t, p)$ when $r \geq c_{0}$, while $r \geq c_{0}$ version of $\left|\alpha_{\mathfrak{J}^{\prime}}+\eta^{\prime}\right| \geq(1 / 4) T_{L}^{-1}$ at $(t, p)$. This implies that $\Phi_{r}$ maps $(\Theta, \hat{\mathfrak{c}})$ and $\left(\Theta, \hat{\mathfrak{c}}^{\prime}\right)$ to distinct gauge equivalence classes.

\section{From pseudoholomorphic curves to Seiberg-Witten instan- tons}

This section with Section 5-7 explain how certain pseudoholomorphic subvarieties in $\mathbb{R} \times M$ can be used to construct instanton solutions to the large $r$ versions of (1-14). These constructions are used in Section 7.k to prove Theorem 1.2. The construction given in what follows of instanton solutions can be viewed as a more complicated version of what was done in the previous section. In particular, all of the steps in Section 3 have their analogs here, but each step is more complicated than its Section 3 analog.

What follows is also much like the construction that is used in the article $\mathrm{Gr} \Rightarrow \mathrm{SW}$ from [5] to construct solutions to the Seiberg-Witten equations from pseudoholomorphic curves in compact, symplectic 4-manifolds. Even as most steps in the construction 
here have their [5] analog, the steps here do differ. This understood, what follows defers to $\mathrm{Gr} \Rightarrow \mathrm{SW}$ in [5] only for the more mundane derivations.

In this section and subsequently, $\Sigma$ denotes a pseudoholomorphic submanifold in $\mathbb{R} \times M$ together with an assigned positive integer weight to each component that has the form $\mathbb{R} \times \gamma$ with $\gamma \subset M$ a Reeb orbit. This integer assignment is ignored when $\Sigma$ is viewed as a submanifold in $M$. It is relevant when viewing $\Sigma$ as a current, however. The purpose of what follows is to construct an instanton solution to any sufficiently large $r$ version of (1-14) from suitably constrained versions of $\Sigma$. These constraints are listed in Section 4.b.

This section mostly sets the stage for the construction. The next two sections complete the construction.

\section{4.a A digression about pseudoholomorphic curves}

Let $C \subset \mathbb{R} \times M$ denote an embedded pseudoholomorphic curve. This section summarizes some of the salient features of $C$ that are used in the subsequent sections. The section has four parts.

Part 1 What with $C$ being a pseudoholomorphic submanifold, there exists $s_{0}>1$ such that the $|s|>s_{0}$ portion of $C$ is a disjoint union of embedded cylinders on which the function $s$ restricts without critical points. Each such cylinder is said to be an end of $C$. This constant $s_{0}$ can be chosen so that the following is true: Suppose first that $\mathcal{E} \subset C$ is an end where $s \ll-1$. There is a Reeb orbit, $\gamma_{\mathcal{E}}$, such that the constant $s$ slices of $\mathcal{E}$ converge uniformly to $\gamma_{\mathcal{E}}$ as $s \rightarrow-\infty$.

To say more about this, remark that the tubular neighborhood map $\varphi: S^{1} \times D \rightarrow M$ for $\gamma_{\mathcal{E}}$ as depicted in (2-1) can be chosen so that the contact form a with the real and imaginary parts of the $\mathbb{C}$-valued 1-form depicted in (3-2) define an orthonormal basis for $T^{*} M$ near $\gamma_{\mathcal{E}}$. Use $\varphi$ to identify $S^{1} \times D$ with a neighborhood of $\gamma$ in $M$. The map $\varphi$ is extended in what is a nonobvious way to identify a neighborhood of $\mathbb{R} \times \gamma_{\mathcal{E}}$ in $\mathbb{R} \times M$ with $\mathbb{R} \times S^{1} \times D$. This extended version of $\varphi$ is the map given by

$$
(w, t, z) \rightarrow\left(s=\frac{\ell_{\gamma}}{2 \pi}\left(w+\frac{1}{2}|z|^{2}\right), \varphi(t, z)\right) .
$$

The latter map is also denoted by $\varphi$ in what follows. The coordinate $w$ is preferred over $s$ because $d w+i d t$ is closer than $d s+i d t$ where $z \neq 0$ to $T^{1,0}(\mathbb{R} \times M)$.

Suppose that $\mathcal{E}$ is not $\mathbb{R}$-invariant. There exists a positive integer $q_{\mathcal{E}}$ and a map, $z_{\mathcal{E}}:\left(-\infty,-s_{0}\right] \times \mathbb{R} /\left(2 \pi q_{\mathcal{E}} \mathbb{Z}\right) \rightarrow \mathbb{C}$ such that $\mathcal{E}$ appears in these coordinates as the image of the map from $\left(-\infty,-s_{0}+1\right] \times \mathbb{R} /\left(2 \pi q_{\mathcal{E}} \mathbb{Z}\right)$ that sends $(w, t)$ to $\left(w, t, z_{\mathcal{E}}(w, t)\right)$. 
This function $z_{\mathcal{E}}$ can be written as

$$
z_{\mathcal{E}}(w, t)=\sum_{q \in \operatorname{div}_{\mathcal{E}}}\left(\varsigma_{q}(t)+\mathfrak{r}_{q}\right) e^{-2 \lambda_{q} w},
$$

where the notation is explained next. To start, introduce $\lambda_{q_{\mathcal{E}}}$ to denote the least negative of the eigenvalues of the set of $2 \pi q_{\mathcal{E}}$ periodic eigenvectors of (2-3). With $\lambda_{q_{\mathcal{E}}}$ so defined, then $\operatorname{div}_{\mathcal{E}} \subset\left\{1,2, \ldots, q_{\mathcal{E}}\right\}$ is the subset with the following two properties: First, any given $q^{\prime} \in \operatorname{div}_{\mathcal{E}}$ is a divisor of $q_{\mathcal{E}}$. Second, there is a $2 \pi q^{\prime}$ periodic eigenvector of (2-3) with eigenvalue $\lambda_{q^{\prime}}$ such that $0>\lambda_{q^{\prime}} \geq \lambda_{q_{\mathcal{E}}}$. What is denoted by $\varsigma_{q}$ in (4-2) is a $\mathbb{C}$-valued function on $\mathbb{R}$ with minimal period $2 \pi q$. Moreover, $\varsigma_{q}$ is a real multiple (perhaps by 0 ) of an eigenvector of the operator $\mathcal{L}$ in (1-2) with $\lambda_{q}$ its eigenvalue. What is denoted by $\mathfrak{r}_{q}$ is a function on $\left(-\infty,-s_{0}+1\right] \times \mathbb{R}$ with period $2 \pi q$ in the $\mathbb{R}$ factor. In addition,

$$
\left|\mathfrak{r}_{q}\right| \leq \varepsilon e^{-\varepsilon|w|}
$$

where $\varepsilon>0$ is a constant.

There is a very much analogous picture of an end $\mathcal{E}$ that lies where $s \gg 1$. Here are the salient differences: First, $z_{\mathcal{E}}$ is now defined on $\left[s_{0}, \infty\right) \times \mathbb{R} /\left(2 \pi q_{\mathcal{E}} \mathbb{Z}\right)$. Second, $\lambda_{q_{\mathcal{E}}}>0$. Finally, a proper divisor $q$ of $q_{\mathcal{E}}$ is in $\operatorname{div}_{\mathcal{E}}$ if and only if the corresponding eigenvector $\lambda_{q}$ obeys $0<\lambda_{q}<\lambda_{q_{\mathcal{E}}}$.

Part 2 This part describes some geometric features of a tubular neighborhood of $C$. To start, let $\pi: N \rightarrow C$ denote the normal bundle to $C$. This bundle has the structure of a holomorphic line bundle with compatible Hermitian structure. The complex structure is determined by $J$ and then the holomorphic and Hermitian structures are induced by the Riemannian metric. An exponential map can be used to identify a tubular neighborhood of any given compact subset of $C$ with small radius disk subbundle in $N$.

It proves convenient to fix an exponential map with certain special features. In particular, there is a constant radius disk subbundle $N_{1} \subset N$ and a smooth map $e_{c}: N_{1} \rightarrow \mathbb{R} \times M$ with five special properties. Here are the first two:

Property 1 The map $e_{C}$ restricts to the zero section as the identity and the differential at the zero section of $e_{C}$ is the identity homomorphism.

Property 2 The map $e_{C}$ is an immersion on $N_{1}$. Moreover, the image via $e_{C}$ of any fiber disk in $N_{1}$ is a pseudoholomorphic disk in $\mathbb{R} \times M$.

Properties 1 and 2 are proved with techniques in [5, Section 5d]'s article SW $\Rightarrow$ Gr. 
The third property refers to the tautological section, $\mathfrak{s}$, of the pullback bundle $\pi^{*} N \rightarrow$ $N$. It also refers to a Hermitian connection, $\theta$, on $\pi^{*} N$. This is the pullback of the induced Hermitian connection on $N \rightarrow C$ that is defined by the Riemannian metric. The latter is also denoted as $\theta$.

Property 3 The pullback via $e_{C}$ of $d s \wedge a+\frac{1}{2} d a$ to any given fiber of $N_{1}$ agrees with the pullback of $(i / 2) \nabla_{\theta} \mathfrak{s} \wedge \nabla_{\theta} \overline{\mathfrak{s}}$ up to an error that has size $O\left(|\mathfrak{s}|^{2}\right)$.

Property 3 is verified using the arguments that are used to prove Lemma 2.2 in the article $\mathrm{Gr} \Rightarrow \mathrm{SW}$ from [5].

The fourth property concerns the behavior of $e_{c}$ on the ends of $C$. To state this property, let $\mathcal{E} \subset C$ denote any given end. As can be seen from (4-2), the vector field vector field $\partial / \partial z$ along $C$ trivializes the normal bundle $\left.N\right|_{\mathcal{E}}$. This trivialization defines the canonical product structure for $\left.N\right|_{\mathcal{E}}$. Use (4-1) and the canonical product structure for $\left.N\right|_{\mathcal{E}}$ to write a point in $\left.N\right|_{\mathcal{E}}$ as $(w, t, z ; \eta)$ with $(w, t, z) \in \mathcal{E}$ and $\eta \in C$.

Property 4 The map $e_{C}$ sends any given point $(w, t, z ; \eta)$ in $\left.N_{1}\right|_{\mathcal{E}}$ to

$$
\left(w+\mathfrak{r}_{w}, t+\mathfrak{r}_{t}, z+\left(1+\mathfrak{r}_{z}\right)\left(\frac{\ell_{\gamma}}{2 \pi}\right)^{-1 / 2} \eta\right),
$$

where $\left|\mathfrak{r}_{w}\right|+\left|\mathfrak{r}_{t}\right| \leq c_{0}\left(|z||\eta|+|\eta|^{3}\right)$ and $\left|\mathfrak{r}_{z}\right| \leq c_{0}|z|^{2}$. In addition, the derivative of $\mathfrak{r}_{w}, \mathfrak{r}_{t}$ and $\mathfrak{r}_{z}$ are uniformly bounded, the former by $c_{0}(|z|+|\eta|)$ and the latter by $c_{0}|z|$.

Given Property 5 below, this property can also be arranged with the help of the techniques from Section $5 \mathrm{~d}$ of the article $\mathrm{SW} \Rightarrow \mathrm{Gr}$ in [5].

The fifth property is relevant only to the $\mathbb{R}$-invariant cylinders. This fifth property implies the first four for such a component. To state this property, let $\gamma \subset M$ denote a Reeb orbit and let $C=\mathbb{R} \times \gamma$. Use the identification given in (4-1) to identify a neighborhood of $C$ in $\mathbb{R} \times M$ with the $z=0$ locus in $\mathbb{R} \times S^{1} \times D$. This identifies the normal bundle of $C$ with $\mathbb{C}$. It also gives $C$ a canonical identification with $\mathbb{R} \times S^{1}$ via the coordinates $(w, t)$. This identification is implicit in what follows. Granted these identifications, here is the fifth property:

Property 5 The exponential map along $\mathbb{R} \times \gamma$ intertwines translation along the respective $\mathbb{R}$ factors of $\mathbb{R} \times \gamma$ and $\mathbb{R} \times M$. In addition it sends $\left.(w, t, \eta) \in N_{1}\right|_{\mathbb{R} \times \gamma} \subset$ $\left(\mathbb{R} \times S^{1}\right) \times \mathbb{C}$ to a point of the form $\left(w+\mathfrak{r}_{w}, t+\mathfrak{r}_{t},\left(\ell_{\gamma} / 2 \pi\right)^{-1 / 2} \eta\right) \in \mathbb{R} \times S^{1} \times D$ where $\left|\mathfrak{r}_{w}\right|+\left|\mathfrak{r}_{t}\right| \leq c_{0}|\eta|^{3}$. 
The existence of an exponential map that obeys Property 5 follows from the fact that the 1 -form $d w+i d t$ differs from an element in $T^{1,0}(\mathbb{R} \times M)$ by $c_{0}|z|^{2}$.

With regards to Property 5: If $a=\left(\ell_{\gamma} / 2 \pi\right)\left(\left(1-\mathrm{R}|z|^{2}\right) d t+(i / 2)(z d \bar{z}-\bar{z} d z)\right.$ in the tubular neighborhood of $\gamma$ with $\mathrm{R}$ constant, and if $d z-i \mathrm{R} d t$ is in $T^{1,0}(\mathbb{R} \times M)$ on this neighborhood, it follows from what is said in [8, Section 2.e] that the exponential map for $\mathbb{R} \times \gamma$ can be chosen so that both $\mathfrak{r}_{w}$ and $\mathfrak{r}_{t}$ are zero.

Part 3 The composition of $e_{c}$ with a section of $N_{1}$ maps $C$ into $\mathbb{R} \times M$. The image is a pseudoholomorphic subvariety if and only if the section obeys a certain nonlinear differential equation. The linearization of this equation along the zero section defines an $\mathbb{R}$-linear, first order differential operator, $\mathcal{D}_{C}$, that extends as a bounded, Fredholm map from $L_{1}^{2}(C ; N)$ to $L^{2}\left(C ; N \otimes T^{0,1} C\right)$. This $\mathcal{D}_{C}$ sends any given section, $\zeta$, of $N$ to

$$
\mathcal{D}_{C} \zeta=\bar{\partial} \zeta+v_{C} \zeta+\mu_{C} \bar{\zeta}
$$

where $v_{C}$ is a section of $T^{0,1} C_{0}$ and where $\mu_{C}$ is one of $N^{2} \otimes T^{0,1} C_{0}$. Note that the parametrization given in (4-1) for any given end of $C$ induces a trivialization of $T C_{0}$ on such an end with the following property: When written using this trivialization and the canonical trivialization of $N$, the pair $\left(v_{C}, \mu_{C}\right)$ converges as $|w| \rightarrow \infty$ on the end to the pair $(\nu, \mu)$ that appears in the associated version of (1-1).

\section{4.b Constraints on $\Sigma$}

Let $\Sigma$ denote a pseudoholomorphic submanifold in $\mathbb{R} \times M$ together with an assigned positive integer weight to each component that has the form $\mathbb{R} \times \gamma$ with $\gamma \subset M$ a Reeb orbit. Various constraints are enforced on $\Sigma$ and on the integral curves of $v$ that are obtained as large $|s|$ limits of the constant $s$ slices of $\Sigma$. These are described in what follows.

The statement of the first four constraints refers to nonnegative integers $m_{\gamma-}$ and $m_{\gamma+}$ that are associated to a given Reeb orbit $\gamma \subset M$. The integer $m_{\gamma-}$ is the sum of the integers from the set $\left\{q_{\mathcal{E}}: \mathcal{E}\right.$ is an $s \ll-1$ end of $\Sigma$ and $\left.\gamma_{\mathcal{E}}=\gamma\right\}$. The integer $m_{\gamma+}$ is the sum of the integer from $\left\{q_{\mathcal{E}}: \mathcal{E}\right.$ is a positive end of $\Sigma$ and $\left.\gamma_{\mathcal{E}}=\gamma\right\}$. Here, $q_{\mathcal{E}}$ in the case where $\mathcal{E}$ is an end of $\mathbb{R} \times \gamma_{\mathcal{E}}$ is the integer that is assigned to $\mathbb{R} \times \gamma_{\mathcal{E}}$ as an element of $\Sigma$.

The following five constraints on $\Sigma$ are assumed in what follows.

Constraint 1 Let $\gamma \subset M$ denote a Reeb orbit with either $m_{\gamma-\text { or }} m_{\gamma+\text { greater }}$ than 1. There is a tubular neighborhood map for $\gamma$ of the sort described in Section 1.a 
and Section 6.a for which the functions $(v, \mu)$ are $\left(v=\frac{1}{2} \mathrm{R}, \mu=0\right)$ with $\mathrm{R} \in \mathbb{R}$ an irrational constant.

The construction of an instanton solution to (1-14) from $\Sigma$ can be made when this constraint is absent, but at considerable cost. [8, Proposition 2.5] can be used to find contact structures all of whose Reeb orbits with any given apriori length bound satisfy this last constraint.

Constraint 2 Let $\mathcal{E}$ denote an end of $\Sigma$ that is not an end of an $\mathbb{R}$-invariant cylinder. Let $q=q_{\mathcal{E}}$ and $\operatorname{div}_{\mathcal{E}}$ denote the data that appears in $\mathcal{E}$ 's version of (4-1). Then $\operatorname{div}_{\mathcal{E}}=\left\{q_{\mathcal{E}}\right\}$ and $\varsigma q_{\mathcal{E}} \neq 0$.

It is also the case that an instant on can be constructed without this constraint, but also at some cost.

Constraint 3 Let $\mathcal{E}$ and $\mathcal{E}^{\prime}$ denote distinct pairs of either $s \ll-1$ or $s \gg 1$ ends of $\Sigma$ that are not part of $\mathbb{R}$-invariant cylinders and are such that $\gamma_{\mathcal{E}}=\gamma_{\mathcal{E}^{\prime}}$ and $q_{\mathcal{E}}=q_{\mathcal{E}^{\prime}}$. Let $q=q_{\mathcal{E}}=q_{\mathcal{E}^{\prime}}$ and let $\varsigma_{q}$ and $\zeta_{q}^{\prime}$ denote the $2 \pi q$-periodic eigenvector that appears in the respective $\mathcal{E}$ and $\mathcal{E}^{\prime}$ versions of (4-1). Then $\varsigma_{q} \neq \eta \varsigma_{q}^{\prime}$ for any $\eta$ such that $\eta^{q}=1$.

The next constraint concerns the operator $\mathcal{D}_{C}$ that is appears in (4-5) for any given component $C \subset \Sigma$.

Constraint 4 Let $C$ denote any given component of $\Sigma$. Then $\mathcal{D}_{C}$ is a Fredholm map from $L_{1}^{2}(C ; N)$ to $L^{2}\left(C ; N \otimes T^{0,1} C\right)$. And, viewed in this light, $\mathcal{D}_{C}$ has trivial cokernel.

Constraints 2-4 hold for any pseudoholomorphic subvariety that is used in the embedded contact homology differential if the contact 1 -form $a$ is in the residual set from [8, Lemma 2.1] and if $J \in \mathcal{J}_{a}$. Note that Constraint 4 enters only in Section 7. It is not required for the constructions in Section 5 and Section 6.

The group $\mathbb{R}$ acts on $\mathbb{R} \times M$ as the constant translations along the $\mathbb{R}$ factor. This being the case, it is convenient to do constructions in an $\mathbb{R}$-equivariant fashion. This is accomplished by first translating $\Sigma$ so that half of the integral of $d a$ over $\Sigma$ is contributed by the $s>0$ part of $\mathbb{R} \times M$ and half by the $s<0$ part. A pseudoholomorphic submanifold with this property is said to be centered. This understood, here is the fifth constraint:

Constraint 5 The submanifold $\Sigma$ is centered. 
The constructions below are done with $\Sigma$ centered. The constructions for a noncentered version of $\Sigma$ are obtained from those that follow by doing the appropriate translation along the $\mathbb{R}$ factor.

\section{4.c Constants}

Fix a pseudoholomorphic subvariety $\Sigma$ with the properties that are listed in Section 4.b. The constructions that follow introduce two $r$-independent constants that are determined by $\Sigma$. The first is denoted by $R$. The constant $R$ should be larger than 1 ; it has a lower bound that is determined by $\Sigma$. This lower bound is chosen, in part, so that the part of $\Sigma$ where $|s| \geq(1 / 100) R$ lies far out on the ends $\Sigma$ 's. Propositions 7.1 and 7.2 give an additional constraint on the lower bound for $R$.

The second constant is denoted by $\sigma$; it must be less than the smaller of $1 / 100$ and a constant that is determined apriori by $\Sigma$ as follows: Let $\mathcal{E}$ denote the set of ends of $\Sigma$. Let $\varepsilon_{\Sigma 1}$ denote the smallest of the versions of the constant $\varepsilon$ that appear in (4-3) for the case when $\mathcal{E} \in \mathcal{E}$ is not part of an $\mathbb{R}$-invariant cylinder. If $\mathcal{E} \in \mathcal{E}$, let $\lambda_{q_{\mathcal{E}}}$ denote the eigenvalue that appears in $\mathcal{E}$ 's version of (4-2). Set $\varepsilon_{\Sigma 2}$ to denote the smallest of those versions of $\left|\lambda_{q_{\mathcal{E}}}-\lambda_{q_{\mathcal{E}^{\prime}}}\right|$ for the cases where $\mathcal{E}$ and $\mathcal{E}^{\prime}$ have the following four properties:

- Both are either negative ends of $\Sigma$, or both are positive ends of $\Sigma$.

- The points on the large $|s|$ slices of both converge as $|s| \rightarrow \infty$ to the same closed integral curve of $v$.

- Neither is part of an $\mathbb{R}$-invariant cylinder.

- $q_{\mathcal{E}} \neq q_{\mathcal{E}^{\prime}}$.

Use $\varepsilon_{\Sigma}$ to denote the minimum of $\varepsilon_{\Sigma 1}$ and $\varepsilon_{\Sigma 2}$. The constant $\sigma$ is further constrained by

$$
\sigma \leq \frac{1}{1000} \varepsilon_{\Sigma} \inf _{\mathcal{E} \in \mathcal{E}}\left|\lambda_{q_{\mathcal{E}}}\right|^{-1}
$$

Positive constants $\rho_{*}, \rho_{\Sigma}, R_{*}$ and a collection $\left\{R_{\mathcal{E}}: \mathcal{E} \in \mathcal{E}\right\}$ are defined in terms of $\sigma$ and $R$ as follows:

$$
\text { - } \rho_{*}=r^{-1 / 2+3 \sigma} \text {. }
$$$$
\text { - } \rho_{\Sigma}=r^{-1 / 2+\sigma} \text {. }
$$

- $R_{*}=100\left(1+\sup _{\mathcal{E} \in \mathcal{E}}\left|\lambda_{q_{\mathcal{E}}}\right|^{-1}\right) \operatorname{lnr}$.

- $R_{\mathcal{E}}=\frac{1}{4}(1-4 \sigma)\left|\lambda_{q_{\mathcal{E}}}\right|^{-1} \ln$ if $\mathcal{E}$ is not part of an $\mathbb{R}$-invariant cylinder.

- $R_{\mathcal{E}}=2 R$ if $\mathcal{E}$ is part of an $\mathbb{R}$-invariant cylinder. 
Note for future reference that these constants are such that when $r$ is large and $\mathcal{E}$ is not part of an $\mathbb{R}$-invariant cylinder, then

- $\rho_{\Sigma} \ll r^{\sigma} \rho_{\Sigma}=e^{-2\left|\lambda_{q_{\mathcal{E}}}\right| \boldsymbol{R}_{\mathcal{E}}}=r^{-\sigma} \rho_{*} \ll \rho_{*}$.

- $e^{-\left(2\left|\lambda_{q_{\mathcal{E}}}\right|+\varepsilon_{\Sigma}\right) R_{\mathcal{E}}} \leq r^{-1 / 2-\sigma} \ll r^{-1 / 2}=r^{-\sigma} \rho_{\Sigma}$.

- $\quad R_{*}>200 \sup _{\mathcal{E} \in \mathcal{E}} R_{\mathcal{E}}$ and $e^{-2\left|\lambda_{q_{\mathcal{E}}}\right| R_{*}} \leq r^{-4}$.

- Suppose that $\mathcal{E}$ and $\mathcal{E}^{\prime}$ obey (4-6) and $\lambda_{q_{\mathcal{E}}}<\lambda_{q_{\mathcal{E}^{\prime}}}<0$. Then $e^{-2\left|\lambda_{q_{\mathcal{E}^{\prime}}}\right| R_{\mathcal{E}}} \leq r^{-1 / 2-\sigma}$.

There is one more "constant" used in what follows, this denoted by $c_{0}$. As in previous sections, it obeys $c_{0} \geq 1$, and its value increases from appearance to appearance. This constant $c_{0}$ is allowed to depend on the choice for $\Sigma$. However, it is independent of $r$. It also lacks dependence on such data as sections of given vector bundles over parts of $\Sigma$ or of parts of $\mathbb{R} \times S^{1}$.

\section{The construction of instantons: Part I}

Fix $\Sigma$ as described in Section 4.b, but do not demand Constraint 4. The purpose of this section is to construct from $\Sigma$ a certain family of pairs consisting of a connection on $E \rightarrow \mathbb{R} \times M$ and a section of $\mathbb{S}=E \oplus E K^{-1} \rightarrow \mathbb{R} \times M$. The curvature of this connection is flat and the $E$-component of the spinor is covariantly constant except very near $\Sigma$.

\section{5.a A connection on $E \rightarrow \mathbb{R} \times M$ and a section of $S \rightarrow \mathbb{R} \times M$}

The constructions that follow use the following notation: The symbols $\mathcal{C}$ and $\mathcal{E}$ denote the respective sets of components of $\Sigma$ and ends of $\Sigma$. When $C \in \mathcal{C}$, then $\mathcal{E}_{C} \subset \mathcal{E}$ denotes the set of ends of $C$. The symbols $\Xi_{\Sigma-}$ and $\Xi_{\Sigma+}$ are used to denote the respective sets of distinct Reeb orbits that are approached as $s \rightarrow-\infty$ and as $s \rightarrow+\infty$ by the constant $s$ slices of $\Sigma$. Thus, $\gamma \in \Xi_{\Sigma-}$ if and only if $\gamma=\gamma_{\mathcal{E}}$ for some $s \ll-1$ end of $\Sigma$. When $\gamma \in \Xi_{\Sigma-}$, the symbol $\mathcal{E}_{\gamma-}$ is used to denote the set of ends $\mathcal{E} \in \mathcal{E}$ with $\gamma_{\mathcal{E}}=\gamma$. There is the analogous definition of $\mathcal{E}_{\gamma+}$ for each $\gamma \in \Xi_{\Sigma+}$.

There are two parts to what follows. The first part describes a relatively simple construction that can be used only in very special circumstances. It is offered as an introduction of sorts to give an indication of what is in store. Part 2 describes the construction that works in the generic situation. Part 2's construction is used in the subsequent sections.

The respective connection and spinor that are constructed below are denoted by $A^{*}$ and $\psi^{*}$. 
Part 1 This part describes a construction of $\left(A^{*}, \psi^{*}\right)$ that can be used in lieu of the construction from Part 2 when the following condition holds:

Let $\mathcal{E}$ denote any given end of $\Sigma$, and let $\gamma=\gamma_{\mathcal{E}}$. If $s \ll-1$ on $\mathcal{E}$, then $m_{\gamma-}=1$; and if $s \gg 1$ on $\mathcal{E}$ then $m_{\gamma+}=1$.

This warm up avoids the complications that arise when the exponential map $\bigcup_{C \in \mathcal{C}} e_{C}$ does not embed any fixed radius disk bundle in $\left.\bigcup_{C \in \mathcal{C}} N\right|_{C}$. This warm up also introduces notation that is used in Part 2 and subsequently. There are two steps to the construction.

Step 1 The bundle $E$ is given by specifying isomorphisms with bundles that are defined over certain sets that comprise an open cover of $\mathbb{R} \times M$. This specification requires one auxiliary parameter, $\rho_{\Sigma}$. In this special case, $\rho_{\Sigma}$ can either be as depicted in (4-8) or independent of $r$. In any event, the parameter $\rho_{\Sigma}$ is positive and is chosen so that vectors in $N$ with norm less than $100 \rho_{\Sigma}$ lie in each $C \in \mathcal{C}$ version of the bundle $N_{1}$. One additional requirement is needed: Given (4-6), there is no generality lost in assuming that the $e_{C}$ and $e_{C^{\prime}}$ images of the respective $100 \rho_{\Sigma}$ radius disk bundles in $\left.N\right|_{C}$ and $\left.N\right|_{C^{\prime}}$ are disjoint when $C \neq C^{\prime}$.

When $k>0$ is given, let $N_{k C}$ denote the radius $k \rho_{\Sigma}$ subbundle in $N_{1}$. The cover of $E$ used here has open sets $\left\{U_{C}=e_{C}\left(N_{4 C}\right)\right\}_{C \in \mathcal{C}}$ and $U_{0}=M-\bigcup_{C \in \mathcal{C}} e_{C}\left(N_{l C}\right)$. Given that (4-6) is assumed, it follows that $U_{C} \cap U_{C^{\prime}}=\varnothing$ when $C \neq C^{\prime}$.

The bundle $E$ restricts to $U_{0}$ as the trivial complex line bundle. Choose an isomorphism $\left.E\right|_{U_{0}}=U_{0} \times \mathbb{C}$. To describe $E$ over $U_{C}$ use the exponential map to identify $U_{C}$ with $N_{1 C}$. This done, $E$ over $N_{1 C}$ is isomorphic to $\pi^{*} N \rightarrow N_{1 C}$ where $\pi: N \rightarrow C$ denotes the projection map.

The transition functions for this cover are as follows: Let $\mathfrak{s}: N_{1 C} \rightarrow \pi^{*} N$ denote the tautological section that sends any given point $\eta \in N_{1 C}$ to $(\eta, \eta) \in \pi^{*} N$. Let $1_{\mathbb{C}}$ denote the constant section of $U_{0} \times \mathbb{C}$ with value $1 \in \mathbb{C}$. Viewed as a section of $E$ over $U_{0}$, the latter appears over $U_{C}=N_{1 C}$ as the section $\mathfrak{s} /|\mathfrak{s}|$.

Step 2 The pair $\left(A^{*}, \psi^{*}\right)$ is specified with respect to the identifications of $E$ given above on the two sets of the open cover of $\mathbb{R} \times M$. Here is the story on $U_{0}$ : The connection $A^{*}$ here is the flat connection from the product structure on $E=U_{0} \times \mathbb{C}$. The latter connection is denoted by $A_{I}$. The section $\psi^{*}$ on $U_{0}$ is given by $\psi_{I}=\left(1_{\mathbb{C}}, 0\right)$.

To define $\left(A^{*}, \psi^{*}\right)$ over $U_{C}=N_{1 C}$, it is necessary to reintroduce the function $\chi$ from Step 2 in Section 3.a. Recall that $\chi: \mathbb{R} \rightarrow[0,1]$ equals 1 on $(-\infty, 5 / 16]$ and equals 0 on $[7 / 16, \infty)$. Set $\chi_{C}$ to denote the function on $N_{1 C}$ given by $\chi\left(|\mathfrak{s}| / \rho_{\Sigma}\right)$ where $\mathfrak{s}$ again denotes the tautological section of the bundle $\pi^{*} N \rightarrow N$. 
It is also necessary to review some of the geometry of the normal bundle. To this end, reintroduce $\theta$ to denote the pullback to $\pi^{*} N$ of the Hermitian connection on $N$ that comes from the Riemannian metric on $\mathbb{R} \times M$. As $\mathfrak{s}$ is nonzero off of the zero section of $N$, there exists a unique connection on $\pi^{*} N$ over $N-0$ for which $\mathfrak{s} /|\mathfrak{s}|$ is covariantly constant; this is the connection

$$
\theta_{\mathfrak{s}}=\theta+\frac{1}{2}\left(\overline{\mathfrak{s}}^{-1} \nabla_{\theta} \overline{\mathfrak{s}}-\mathfrak{s}^{-1} \nabla_{\theta} \mathfrak{s}\right)
$$

Introduce the map $\widehat{r}: N \rightarrow N$ that is defined by setting $\widehat{r}(\eta)=r^{1 / 2} \eta$. As $\pi(r \eta)=\pi(\eta)$, there is a canonical isomorphism between $\hat{r}^{*} \pi^{*} N$ and $\pi^{*} N$. With this isomorphism understood, $\hat{r}^{*} \theta=\theta$.

To end these preliminaries, introduce the $E=N$ version of the fiber bundle $\mathfrak{C}_{E, 1} \rightarrow C$ that is introduced in Section 2.e. The latter is denoted as $\mathfrak{C}_{N, 1}$. Let $\mathfrak{c} \in C^{\infty}\left(C ; \mathfrak{C}_{N, 1}\right)$ denote the section that assigns the unique symmetric vortex to each point in $C$. Lift $\mathfrak{c}$ as a pair $\left(\mathbb{A}^{C}, \alpha^{C}\right)$ where $\mathbb{A}^{C}$ is a connection on the bundle $\pi^{*} N \rightarrow N$ and $\alpha^{C}$ is a section, this as described in Section 2.g. In particular, choose this lift that $\alpha^{C}=$ $\left|\alpha^{C}\right| \mathfrak{s} /|\mathfrak{s}|$. Note that this lift insures that the unique solution $A_{0}$ to (2-32) is zero. Thus, $\mathbb{A}^{C}=\theta_{\mathfrak{s}}+\mathbb{A}^{C}$ where $\mathbb{A}^{C}$ annihilates the horizontal vectors in $N$. Use $\left(\mathbb{A}^{C, r}, \alpha^{C, r}\right)$ to denote $\hat{r}^{*}\left(\mathbb{A}^{C}, \alpha^{C}\right)$. Granted all of this notation, define $\left(A^{*}, \psi^{*}\right)$ on $U_{C}$ by

- $A^{*}=\left(1-\chi_{C}\right) \theta_{\mathfrak{s}}+\chi_{C} \mathbb{A}^{C, r}$.

- $\psi^{*}=(\alpha, \beta)$ with $\alpha=\chi_{C} \alpha^{C, r}+\left(1-\chi_{C}\right) \mathfrak{s} /|\mathfrak{s}|$ and $\beta=0$.

Part 2 This part gives the construction that is ultimately used in what follows. Note that even in the case when Part 1's assumption holds, the resulting $\left(A^{*}, \psi^{*}\right)$ differs somewhat from that in (5-2).

The construction here requires the parameters $R, \rho_{*}, \rho_{\Sigma}$ and the collection $\left\{R_{\mathcal{E}}\right\}_{\mathcal{E} \in \mathcal{E}}$ as given in (4-8). When $k$ is a positive integer and $C \in \mathcal{C}$, use $N_{k C} \rightarrow C$ to again denote the radius $k \rho_{\Sigma}$ subbundle in $N$. When $k$ is a nonnegative integer and $\mathcal{E}$ is an end of $\Sigma$, use: $\mathcal{E}_{k R} \subset \mathcal{E}$ to denote the part of $\mathcal{E}$ where $|s| \geq R_{\mathcal{E}}+k R$. It is assumed implicitly that $\bigcup_{C \in \mathcal{C}} e_{C}$ embeds the restriction of $\bigcup_{C \in \mathcal{C}} N_{100 C}$ to $\bigcup_{C \in \mathcal{C}}\left(C-\left(\bigcup_{\mathcal{E} \in \mathcal{E}_{C}} \mathcal{E}_{100 R}\right)\right)$. Note that such is the case when $r$ is large. What with Constraints 2 and 3 in Section 4.b, this follows directly using (4-8), (4-9) and (4-2).

There are two steps to the construction of the desired pair $\left(A^{*}, \psi^{*}\right)$.

Step 1 The bundle $E$ is again given by specifying isomorphisms with bundles that are defined over sets that comprise an open cover of $\mathbb{R} \times M$. The cover of $\mathbb{R} \times M$ 
used here consists of the following:

- A collection $\left\{U_{C}\right\}_{C \in \mathcal{C}}$ where any given $U_{C}$ is the image via $e_{C}$ of the restriction of $N_{4 C}$ to $C-\left(\bigcup_{\mathcal{E} \in \mathcal{E}_{C}} \mathcal{E}_{2 R}\right)$.

- A set $U_{\gamma-}$ for each $\gamma \in \Xi_{\Sigma-}$ and a set $U_{\gamma+}$ for each $\gamma \in \Xi_{\Sigma+}$. The set $U_{\gamma}$ - consists of the portion of $\gamma$ 's version of $\mathbb{R} \times S^{1} \times D$ where both $w<-2 R$ and the distance to $\gamma$ along $M$ at constant $w \in R$ is

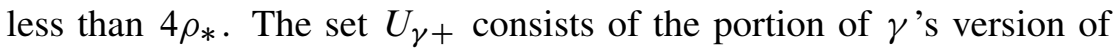
$\mathbb{R} \times S^{1} \times D$ where both $w>2 R$ and the distance to $\gamma$ along $M$ at constant $w \in R$ is less than $4 \rho_{*}$.

- A set $U_{0}$. This is the complement in $\mathbb{R} \times M$ of the closure of a union of sets, one labeled by $\Sigma$, one labeled by each $\gamma \in \Xi_{\Sigma-}$ and one labeled by each $\gamma \in \Xi_{\Sigma+}$. The set labeled by $\Sigma$ is the image via $e_{\Sigma}$ of the restriction of $N_{\Sigma}$ to $\Sigma-\left(\bigcup_{\mathcal{E} \in \mathcal{E}} \mathcal{E}_{4 R}\right)$. The set labeled by $\gamma \in \Xi_{\Sigma-}$ is the portion of $\gamma$ 's version of $\mathbb{R} \times S^{1} \times D$ where both $w<-4 R$ and the distance to $\gamma$ along $M$ at constant $w \in R$ is less than $\rho_{*}$. The set $U_{\gamma+}$ is the portion of $\gamma$ 's version of $\mathbb{R} \times S^{1} \times D$ where both $w>4 R$ and the distance along $M$ at constant $w \in R$ is less than $\rho_{*}$.

Note that $U_{C} \cap U_{C^{\prime}}=\varnothing$ when $C \neq C^{\prime}$. By the same token, $U_{\gamma-} \cap U_{\gamma^{\prime}}$ and $U_{\gamma+} \cap U_{\gamma^{\prime}+}=\varnothing$ when $\gamma \neq \gamma^{\prime}$. Finally, $U_{\gamma-} \cap U_{\gamma^{\prime}+}=\varnothing$ in all cases.

The bundle $E \rightarrow M$ is given below as a product bundle over $U_{0}$ and over each set from $\left\{U_{\gamma-}\right\}_{\gamma \in \Xi_{\Sigma-}}$ and $\left\{U_{\gamma+}\right\}_{\gamma \in \Xi_{\Sigma+}}$. Over any given $U_{C}$, it is given as follows: Let $C \subset \Sigma$ denote a non- $\mathbb{R}$-invariant component. The bundle $E$ over $U_{C}$ is given as $\pi^{*} N$. In the case where $C$ is an $\mathbb{R}$-invariant cylinder, the bundle $E$ is formally $\pi^{*} N^{q}$ where $q$ is $C$ 's associated integer. However, the normal bundle of any given version of $\mathbb{R}$-invariant cylinder has the trivialization described by Property 5 in Section 4.a; this trivialization canonically identifies $E$ over $\mathbb{C}$ 's component of $U_{C}$ with the product bundle. The next three paragraphs describe the transition functions that relate the respective incarnations of $E$ over the intersection of distinct sets from the open cover.

The transition function for the intersection between $U_{0}$ and $U_{C}$ is as follows: In the case where $C$ is not $\mathbb{R}$-invariant, the constant section $1_{\mathbb{C}}$ over $U_{0}$ appears as the section $\mathfrak{s} /|\mathfrak{s}|$ on $U_{C}$. In the case where $C=\mathbb{R} \times \gamma$, the constant section $1_{\mathbb{C}}$ over $U_{0}$ appears as $z^{q} /|z|^{q}$ on $U_{C}$ where $q$ is the integer assigned to $C$. Here, the neighborhood of $C=\mathbb{R} \times \gamma$ is identified with $\mathbb{R} \times\left(S^{1} \times D\right)$ using $\gamma$ 's version of the tubular neighborhood map $\varphi$ as described in (4-1).

What follows describes the transition function for $U_{0} \cap U_{\gamma-}$. The transition function for any $U_{0} \cap U_{\gamma+}$ has a completely analogous description and is not given. To start 
the description, remark that the $s \ll-1$ part of $\Sigma$ intersects the image of any constant $(w, t)$ disk in $(-\infty,-R) \times S^{1} \times D$ as a set of distinct points. This follows from (4-2). To elaborate, each end $\mathcal{E} \subset \mathcal{E}_{\gamma-}$ that is not part of an $\mathbb{R}$-invariant cylinder contributes $q_{\mathcal{E}}$ points to this intersection, and then there is one additional point with $z=0$ if $\Sigma$ contains the cylinder $\mathbb{R} \times \gamma$. The $z=0$ point should be viewed as a point with multiplicity $q$, where $q$ is the integer that is assigned to this cylinder. In this

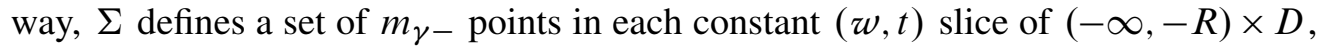
with all but possibly the point $z=0$ distinct. As $w$ and $t$ vary, this set of weighted points varies and so defines a map $(w, t) \rightarrow \mathfrak{Z}_{\Sigma \gamma-}(w, t) \in \operatorname{Sym}^{m_{\gamma-}}(\mathbb{C})$. Granted this notation, the transition function is such that the trivializing section of $E$ 's restriction to $U_{0}$ appears as the function on $(-\infty,-R) \times S^{1} \times D$ given by

$$
(w, t, z) \rightarrow \prod_{z^{\prime} \in \mathcal{Z}_{\Sigma} \gamma_{-}(w, t)} \frac{\left(z-z^{\prime}\right)}{\left|z-z^{\prime}\right|}
$$

with respect to the trivializing section for $E$ 's restriction to $U_{\gamma-}$. This map is smooth on the domain $\left(\mathbb{R} \times S^{1} \times D\right) \cap U_{0}$ by virtue of the fact that distinct $z \neq 0$ points remain distinct (and nonzero) as $(w, t)$ vary.

Consider next the intersection between $U_{\gamma-}$ and $U_{C}$. The normal bundle to $C$ in $U_{\gamma-}$ is isomorphic to the trivial bundle, $C \times C$. Use the coordinates $(w, t, z)$ for $U_{\gamma-}$. As noted in Section 4.a, there is a trivialization of $N$ over $C \cap U_{\gamma-}$ with the property that the exponential map $e_{C}$ sends any given point $(w, t, z) \in C$ and $\eta \in N_{1}$ to what is written in (4-4). This gives a trivialization of $E$ over the part of $U_{C}$ in $U_{\gamma-}$ since $E$ over any given component of $U_{C}$ is an appropriate tensor power of $\pi^{*} N$. With respect to this trivialization of $E$ over $U_{C} \cap U_{\gamma} \subset \subset U_{C}$, the trivializing section of $E$ over $U_{\gamma-}$ appears on $U_{C}$ as the function that sends a point with coordinates $(w, t, z) \in C$ and $\eta \in N_{1}$ to

$$
\left(\prod_{\substack{z^{\prime \prime} \neq \widehat{z}, z^{\prime \prime} \in \mathfrak{Z}_{\Sigma \gamma-}\left(w^{\prime}, t^{\prime}\right)}} \frac{\left(z^{\prime}-z^{\prime \prime}\right)}{\left|z^{\prime}-z^{\prime \prime}\right|}\right)^{-1}\left(\frac{z^{\prime}-\widehat{z}}{\left|z^{\prime}-\widehat{z}\right|}\right)^{-1} \frac{\eta}{|\eta|} .
$$

Here the notation is as follows: First, the triple $\left(w^{\prime}, t^{\prime}, z^{\prime}\right)$ is $\left(w+\mathfrak{r}_{w}, t+\mathfrak{r}_{t}, z+\right.$ $\left.\left(1+\mathfrak{r}_{z}\right)\left(\ell_{\gamma} / 2\right)^{-1 / 2} \eta\right)$ with $\mathfrak{r}_{w}, \mathfrak{r}_{t}$ and $\mathfrak{r}_{z}$ as given in (4-4). Second, $\widehat{z}$ is the point that is closest to $z$ in the $\left(w^{\prime}, t^{\prime}\right)$ slice of $C$. Note that there is a unique such point when $\eta \in N_{1}$ and that the assignment of this point to the point $(w, t, z) \in C$ varies smoothly.

There is a similar picture of the transition functions for $\left\{U_{\gamma+} \cap U_{C}\right\}_{\gamma \in \Xi_{\Sigma+}}$.

Step 2 This step describes $\left(A^{*}, \psi^{*}\right)$ on the sets given in (5-2). Start with $U_{0}$. Given that the bundle $E$ here has the product structure $U_{0} \times \mathbb{C}$, set $\left(A^{*}, \psi^{*}\right)=\left(A_{I},\left(1_{\mathbb{C}}, 0\right)\right)$ 
where $A_{I}$ is the flat connection given by the product structure. This pair $\left(A_{I},\left(1_{\mathbb{C}}, 0\right)\right)$ is sometimes denoted by $\left(A_{0}, \psi_{0}\right)$.

The pair $\left(A^{*}, \psi^{*}\right)$ on $U_{C}$ is given by (5-2) on $C$ 's component of $U_{C}$ when $C \in \mathcal{C}$ is not $\mathbb{R}$-invariant. Suppose next that $C=\mathbb{R} \times \gamma$ with associated integer $q$. The definition here is that given in (3-2) save that $\chi_{C}$ should be used in place of $\chi_{*}$. To elaborate, let $\mathfrak{c} \in C^{\infty}\left(\mathbb{R} \times S^{1} ; \mathfrak{C}_{q}\right)$ denote the constant map to the unique symmetric vortex. Lift $\mathfrak{c}$ as a pair $\left(A^{C}, \alpha^{C}\right)$ where $A^{C}$ is a connection on the trivial bundle over $\mathbb{C}$ and $\alpha^{C}$ is a section. There is a unique lift for which $\alpha^{C}=\left|\alpha^{C}\right| z^{q} /|z|^{q}$. Reintroduce $r_{\gamma}=\left(\ell_{\gamma} /(2 \pi)\right) r$ and also the rescaling map $\hat{r}_{\gamma}: R \times S^{1} \times \mathbb{C} \rightarrow \mathbb{R} \times S^{1} \times \mathbb{C}$ to denote the map that rescales the $\mathbb{C}$ factor via $z \rightarrow r_{\gamma}^{1 / 2} z$. Use $\left(A^{C, r}, \alpha C, r\right)$ to denote $\widehat{r}_{\gamma}^{*}\left(A^{C}, \alpha^{C}\right)$. Let $\theta$ denote the connection on the product bundle over $\left(\mathbb{R} \times S^{1} \times \mathbb{C}\right)$ that is induced by the product structure. With this notation understood, $A^{*}$ on $U_{C}$ is given by $A^{*}=\left(1-\chi_{C}\right)\left(\theta+\frac{1}{2} q\left(\bar{z}^{-1} d \bar{z}-z^{-1} d z\right)\right)+\chi_{C} A^{C, r}$, and $\psi^{*}$ on $U_{C}$ is given by $(\alpha, \beta)$ with $\alpha=\chi_{C} \alpha^{C, r}+\left(1-\chi_{C}\right) z^{q} /|z|^{q}$ and $\beta=0$.

Now consider $\left(A^{*}, \psi^{*}\right)$ on a given set from the collection $\left\{U_{\gamma-}\right\}_{\gamma \in \Xi_{\Sigma-}}$. Note that the description on a given set from $\left\{U_{\gamma+}\right\}_{\gamma \in \Xi_{\Sigma+}}$ is identical save for some sign changes. To start the description on $U_{\gamma-}$, remark that the points that comprise $r_{\gamma}^{1 / 2} \mathfrak{Z}_{\Sigma \gamma}$ - define a map from $(-\infty,-R) \times S^{1}$ to $\operatorname{Sym}^{m_{\gamma-}}(\mathbb{C})$ and thus a map, $\mathfrak{c}_{\gamma-}:(-\infty,-R) \times S^{1} \rightarrow$ $\mathfrak{C}_{m_{\gamma-}}$. This is a smooth map since the nonzero points that comprise $\mathfrak{Z}_{\Sigma \gamma-}$ are distinct

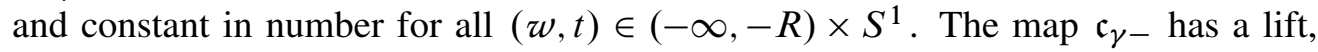
$\left(\mathbb{A}^{\gamma-}, \alpha^{\gamma-}\right)$, as a pair that consists of a connection and section for the trivial bundle over $(-\infty,-R) \times S^{1} \times \mathbb{C}$, these pulling-back on each constant $(w, t)$ slice so as to solve (1-4). In particular, there is a lift with the following properties:

- $\alpha^{\gamma-}=\left|\alpha^{\gamma-}\right| \prod_{z^{\prime} \in \mathfrak{Z}_{\Sigma \gamma-}}\left(\left(z-r_{\gamma}^{1 / 2} z^{\prime}\right) /\left|z-r_{\gamma}^{1 / 2} z^{\prime}\right|\right)$ at points with $|z| \geq 1+2 \sup \left\{r_{\gamma}^{1 / 2}\left|z^{\prime}\right|:\left.z^{\prime} \in \mathfrak{Z}_{\Sigma \gamma-}\right|_{(w, t)}\right\}$

- $\alpha^{\gamma-}=e^{u} \alpha_{0}^{0} \prod_{0 \neq z^{\prime} \in \mathfrak{Z}_{\Sigma \gamma-}} \alpha_{z^{\prime}}^{t}$, at points with $w \geq-4 R$. Here,

(a) $\alpha_{z^{\prime}}^{t}=\left|\alpha_{z^{\prime}}^{t}\right|\left(z-r_{\gamma}^{1 / 2} z^{\prime}\right) /\left|z-r_{\gamma}^{1 / 2} z^{\prime}\right|$ is the $n=l$ vortex with center at $z^{\prime}$ if $z^{\prime} \neq 0$.

(b) $\alpha_{0}^{0}=\left|\alpha_{0}^{0}\right| z^{q} /|z|^{q}$ is the $n=q$ symmetric vortex if $\mathbb{R} \times \gamma$ is a component of $\Sigma$ with $q$ here the associated integer. Otherwise, $\alpha_{0}^{0}=1$.

(c) $|\operatorname{Re}(u)| \leq c_{0} \sum_{z^{\prime} \neq z^{\prime \prime} ; z^{\prime}, z^{\prime \prime} \in \mathfrak{Z}_{\Sigma \gamma-}} e^{-\sqrt{r_{\gamma}}\left|z^{\prime}-z^{\prime \prime}\right| / 2}$.

- $\mathbb{A}^{\gamma-}=\theta_{0}+A_{0}^{\gamma-}+\mathbb{A}^{\gamma-}$, where $\theta_{0}$ is the connection from the product structure $A^{\gamma}$ is an $i$-valued 1 -form that annihilates the tangent vectors to the $\mathbb{R} \times S^{1}$ factor and $A_{0}^{\gamma-}$ annihilates the tangent vectors to the $\mathbb{C}$ factor and is given by (2-32). 
Use $\left(\mathbb{A}^{\gamma-, r}, \alpha^{\gamma-, r}\right)$ to denote the pullback via $\hat{r}_{\gamma}$ of the pair $\left(\mathbb{A}^{\gamma-}, \alpha^{\gamma-}\right)$. With the latter in hand, the definition of $\left(A^{*}, \psi^{*}\right)$ is a relatively straightforward operation. Over the part of $U_{\gamma-}$ where both $|z|<\frac{1}{4} \rho_{*}$ and $w<-4 R$, the pair $\left(A^{*}, \psi^{*}\right)=$ $\left(\mathbb{A}^{\gamma-, r},\left(\alpha^{\gamma-, r}, 0\right)\right)$. The definition where either of these two constraints are violated uses bump functions and the relevant transition functions to patch the latter with what has been defined previously.

To say more about this patching business, it is first necessary to specify the bump functions that are involved. The first, $\chi_{\gamma_{-}}$, is the function on the $\mathbb{C}$ factor in $\mathbb{R} \times S^{1} \times \mathbb{C}$ that sends $z$ to $\chi\left(|z| \rho_{*}\right)$. The second is a function $\chi_{R-}$ on $\mathbb{R} \times\left(S^{1} \times D\right)$ that depends only on the $\mathbb{R}$ factor; it is given by $\chi_{R_{-}}(w)=\chi((3 R+w) / R)$. An analogous function $w \rightarrow \chi_{R+}(w)=\chi((3 R-w) / R)$ is used for the $U_{\gamma+}$ versions of the formulae that follow.

Consider first the patching on the part of $U_{\gamma-}$ that lies where $|z| \geq \frac{1}{4} \rho_{*}$ or $w \geq-4 R$, but in either case where the distance to $\Sigma$ is $\rho_{\Sigma}$ or greater. Introduce for this purpose the $S^{1}$ valued function

$$
(w, t, z) \rightarrow u_{\gamma-}=\prod_{\left.z^{\prime} \in \mathfrak{Z}_{\gamma-1}\right|_{(w, t)}} \frac{\left(z-z^{\prime}\right)}{\left|z-z^{\prime}\right|} .
$$

Define $\left(A^{*}, \psi^{*}\right)$ on the part of $U_{\gamma-}$ under consideration by

$$
\begin{aligned}
& \text { - } A^{*}=\left(1-\chi_{\gamma-} \chi_{R-}\right)\left(\theta_{0}-u_{\gamma-}^{-1} d u_{\gamma-}\right)+\chi_{\gamma-\chi_{R-}} \mathbb{A}^{\gamma-, r} . \\
& \text { - } \psi^{*}=\left(\left(1-\chi_{\gamma-} \chi_{R-}\right) u_{\gamma-}+\chi_{\gamma-\chi_{R-}} \alpha^{\gamma-, r}, 0\right) .
\end{aligned}
$$

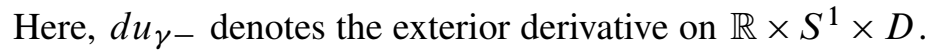

Consider next the patching business for the points in $U_{\gamma-}$ with distance less than $\rho_{\Sigma}$ from $\Sigma$. To start, let $\mathcal{E} \subset \Sigma$ denote an end where $s \ll-1$ and with $\gamma_{\mathcal{E}}=\gamma$. Use the transition function that is depicted in (5-5) to write the $U_{\Sigma}$ version of the pair $\left(A^{*}, \psi^{*}\right)$ with respect to the trivialization of $E$ over the part of $U_{\Sigma}$ in $U_{\gamma-}$ as the product bundle. Write the result using the coordinates $(w, t, z)$ as $\left(A^{\mathcal{E}},\left(\alpha^{\mathcal{E}}, 0\right)\right)$. Another cut-off function on $\mathbb{R} \times\left(S^{1} \times D\right)$ is required for the upcoming formula. It is denoted by $\chi_{\mathcal{E}}$ and it is defined to be $\chi\left(\left(R_{\mathcal{E}}+3 R-|w|\right) / R\right)$. With these preliminaries set, define $\left(A^{*}, \psi^{*}\right)$ where $U_{\gamma-}$ intersects $U_{\Sigma}$ near $\mathcal{E}$ by

$$
\text { - } A^{*}=\chi_{\mathcal{E}}\left(\left(1-\chi_{\gamma-}\right)\left(\theta_{0}-u_{\gamma-}^{-1} d u_{\gamma-}\right)+\chi_{\gamma-\mathbb{A}^{\gamma-, r}}\right)+\left(1-\chi_{\mathcal{E}}\right) \mathbb{A}^{\mathcal{E}} \text {. }
$$

- $\psi^{*}=(\alpha, \beta)$ with $\alpha=\chi_{\mathcal{E}}\left(\left(1-\chi_{\gamma-}\right) u_{\gamma-}+\chi_{\gamma-} \alpha^{\gamma-, r}\right)+\left(1-\chi_{\mathcal{E}}\right) \alpha^{\mathcal{E}}$ and $\beta-0$. 


\section{5.b A family of deformations of $\left(A^{*}, \psi^{*}\right)$}

The desired family is parametrized by elements from a certain subset of a Banach space to be defined momentarily. The pair of connection and spinor labeled by a given element $\xi$ from this ball is denoted here by $\left(A^{\xi}, \psi^{\xi}\right)$. The construction of $\left(A^{\xi}, \psi^{\xi}\right)$ has seven steps.

Step 1 Let $\Theta_{-}=\left\{\left(\gamma, m_{\gamma-}\right)\right\}_{\gamma \in \Xi_{\Sigma-}}$ and let $\Theta_{+}=\left\{\left(\gamma, m_{\gamma+}\right)\right\}_{\gamma \in \Xi_{\Sigma+}}$. The constructions in Section 3 can be carried out using the chosen coordinate charts for the relevant Reeb orbits, the constant $\rho_{*}$ and $\Theta=\Theta_{-}$. Lemma 3.8 supplies $\zeta_{-}=\left\{\zeta_{\gamma-}\right\}_{\gamma \in \Xi_{\Sigma-}}$. Lemma 3.8 likewise supplies $\zeta_{+}=\left\{\zeta_{\gamma+}\right\}_{\gamma \in \Xi_{\Sigma+}}$.

Step 2 This step serves as a warm up to describe the desired family when the assumption in Part 1 of Section 5.a holds and $\left(A^{*}, \psi^{*}\right)$ is given by (5-2) using the construction from Part 1 of Section 5.a. The Banach space is $\bigoplus_{C \in \mathcal{C}} \mathcal{K}_{\mathfrak{c} *}$ with $\mathfrak{c}$ here denotes the section that assigns the symmetric vortex to each point in $C$ and with the $\mathcal{K}_{\mathfrak{c} *}$ defined subsequent to (2-27). This is to say that it is the completion of the space of smooth, compactly supported sections of $\mathfrak{c}^{*} V_{1,0} \mathfrak{C}_{N, 1}$ using the norm whose square is depicted by the middle item in (2-27). Note that the identification between $\mathfrak{C}_{1}$ and $\mathbb{C}$ given by the function $\sigma_{1}$ in (1-5) gives the following equivalent definition: The space $\mathcal{K}_{\mathfrak{c} *}$ is the completion of the space of smooth, compactly supported sections of the normal bundle to $C$ using the norm whose square is depicted in the middle item of (2-27) with $\nabla$ taken to be the connection that is induced by the Levi-Civita connection of the metric on $\mathbb{R} \times M$. Viewed this way, an element $\xi \in \bigoplus_{C \in \mathcal{C}} \mathcal{K}_{\mathfrak{c} *}$ has components $\left(\xi_{C}\right)_{C \in \mathcal{C}}$ where $\xi_{C}$ is a section of $N \rightarrow C$.

The relevant subset in $\bigoplus_{C \in \mathcal{C}} \mathcal{K}_{\mathfrak{c} *}$ is denoted in what follows by $\mathcal{K}_{*}$; it is the subspace with the property that each component of any given $\xi \in \mathcal{K}_{*}$ has pointwise norm less than a constant $\varepsilon_{0}$ with $\varepsilon_{0}>0$ chosen so that the map êxp from Part 8 of Section 2.a embeds the radius $10^{6} \varepsilon_{0}$ disk in each fiber of $T_{1,0} \mathfrak{C}_{1}$. A second Banach space also plays a role here, this being $\mathcal{K}=\bigoplus_{C \in \mathcal{C}} \mathcal{K}_{\mathfrak{c}}$. Here, $\mathcal{K}_{\mathfrak{c}} \subset \mathcal{K}_{\mathfrak{c} *}$ is the set of elements on which the norm in the third item of $(2-27)$ has a finite value.

Some further preliminaries are needed to define the pair $\left(A^{\xi}, \psi^{\xi}\right)$ of connection on $E$ and section of $\mathbb{S}$. To start, keep in mind that $N$ on any given end $\mathcal{E} \subset \Sigma$ has a trivialization that is supplied by the coordinate chart associated to the Reeb orbit $\gamma_{\mathcal{E}}$. Indeed, $\mathcal{E}$ is transversal to the constant $(w, t)$ disks in the neighborhood $\mathbb{R} \times S^{1} \times D$ of $\gamma_{\mathcal{E}}$, and so the vector field $\partial / \partial z$ tangent to the $D$ factor trivializes $N$. This trivialization allows a section of $N$ on $\mathcal{E}$ to be viewed as a smooth map from $\mathcal{E}$ to $\mathbb{C}$. Moreover, since the projection from $\mathbb{R} \times S^{1} \times D$ to $\mathbb{R} \times S^{1}$ restricts to $\mathcal{E}$ as a 1-1 map, 
so a section of $N$ on $\mathcal{E}$ can be viewed as a map from $\mathbb{R} \times S^{1}$ to $\mathbb{C}$. With the preceding understood, if $\mathcal{E}$ is an end where $s \ll-1$, then the $\mathbb{R}$-independent map given by the

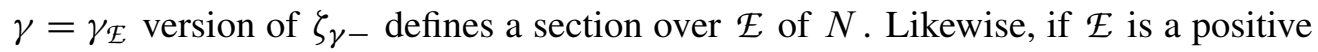
end, then the map given by the $\gamma=\gamma_{\mathcal{E}}$ version of $\zeta_{\gamma+}$ defines a section over $\mathcal{E}$ of $N$. In either case, these are to be viewed as sections over $\mathcal{E}$ of $\mathfrak{c}^{*} V_{1,0} \mathfrak{C}_{N, 1}$. In either case, these sections have norm bounded by $c_{0} r^{-1 / 2}$.

Fix a section, $\zeta_{*}$, of the bundle $\bigoplus_{C \in \mathcal{C}} \mathfrak{c}^{*} V_{1,0} \mathfrak{C}_{N, 1}$ with the following properties: First, it is equal where $s \ll-1$ on any given negative end: $\mathcal{E}$ to the $\gamma=\gamma_{\mathcal{E}}$ version of $\zeta_{\gamma-}$ and equal where $s \gg 1$ on any given positive end $\mathcal{E}$ to the $\gamma=\gamma_{\mathcal{E}}$ version of $\zeta_{\gamma+}$. Second, it has support where $|s| \gg R$ on the ends of $\Sigma$. Note that there exist such sections with $L_{1}^{2}$ and supremum norm bounded by $2 c_{0} r^{-1 / 2}$. Fix $\zeta_{*}$ with this last property also. It is a consequence of Lemma 3.10 that $\zeta_{*}$ can be chosen so that

$$
\sup _{p \in \Sigma, \rho<1} \rho^{-v} \int_{\operatorname{dist}(\cdot, p)<\rho}\left|\nabla \zeta_{*}\right|^{2} \leq c_{0} r^{-1 / 2} .
$$

Here, $\nabla$ is defined by the pullback via $\mathfrak{c}$ of the Levi-Civita connection. With regards to (5-10), note that this bound holds with $v=1$ for $\zeta_{\gamma-}$ because the integral in question is

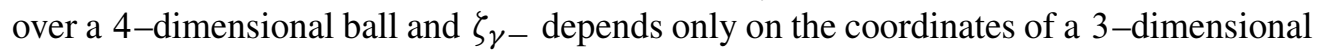
slice. The bound in (5-10) is assumed in what follows.

With these preliminaries set, fix $\xi=\left(\xi_{C}\right)_{C \in \mathcal{C}} \in \mathcal{K}_{*}$. Let $C \in \mathcal{C}$ and define the pair $\left(\mathbb{A}^{C \xi}, \alpha^{C \xi}\right)$ of connection on $E=\pi^{*} N \rightarrow N$ and section of $E$ over $N$ by using (2-36) with $\left(\mathbb{A}^{C}, \alpha^{C}\right)$ in lieu of $(A, \alpha)$ and $\zeta_{*}+\xi_{C}$ in lieu of what is called $\zeta$ in (2-36). Rescale with the map $\hat{r}$ and denote the result as $\left(\mathbb{A}^{C \xi, r}, \alpha^{C \xi, r}\right)$. Write the pair $\left(\mathbb{A}^{C \xi, r}, \psi=\left(\alpha^{C \xi, r}, 0\right)\right)$ of connection on $E \rightarrow U_{C}$ and section of $\mathbb{S}$ over $U_{C}$ as $\left(A^{*}, \psi^{*}\right)+\mathfrak{t}^{C \xi}$ where $\mathfrak{t}^{C \xi}$ is a section over $U_{C}$ of $i T^{*} M \oplus \mathbb{S}$ with zero component in the $E K^{-1}$ summand of $\mathbb{S}$. The desired pair of connection on $E$ and section of $\mathbb{S}$ in this case is

$$
\left(A^{\xi}, \psi^{\xi}\right)=\left(A^{*}, \psi^{*}\right)+\sum_{C \in \mathcal{C}} \chi_{C} \mathfrak{t}^{C \xi}
$$

Step 3 This step starts the story in the general case by setting the stage and notation. If $C \in \mathcal{C}$ is a not $\mathbb{R}$-invariant, let $\mathfrak{c} \in C^{\infty}\left(C ; \mathfrak{C}_{N, 1}\right)$ denote the section that assigns to each point the symmetric vortex and set $V_{C}=\mathfrak{c}^{*} V_{1,0} \mathfrak{C}_{N, 1}$. If $C \in \mathcal{C}$ is $\mathbb{R}$-invariant, let $q$ denote its associated integer and use $V_{C}$ to denote the product bundle $C \times \mathbb{C}^{q}$.

Let $\gamma \in \Xi_{\Sigma-}$ and reintroduce the map $\mathfrak{c}_{\gamma_{-}}:(-\infty,-R] \times S^{1} \rightarrow \mathfrak{C}_{m_{\gamma-}}$ from the preceding subsection. What is said in Part 5 of Section 2.a can be used to define a homomorphism from $\times_{C \in \mathcal{C}} C^{\infty}\left(\left.C\right|_{|w|<R_{*}} ; V_{C}\right)$ to $C^{\infty}\left(\left(-R_{*},-R\right) \times S^{1} ; \mathfrak{c}_{\gamma-}^{*} T_{1,0} \mathfrak{C}_{m_{\gamma}}\right)$. This homomorphism is obtained as follows: Write $\mathfrak{c}_{\gamma-}$ as $\left(\mathbb{A}^{\gamma-}, \alpha^{\gamma-}\right)$ and write $\alpha^{\gamma-}$ 
as in the second bullet of (5-6). Let $C \in \mathcal{C}$ denote a component that is not $\mathbb{R}$-invariant and let $\mathcal{E} \subset C$ denote an end that intersects $U_{\gamma-}$. A point $\left(w, t, z^{\prime}\right) \in \mathcal{E}$ defines the zero at $r_{\gamma}^{1 / 2} z^{\prime}$ of the function $\alpha^{\gamma-}$; this is the zero of the factor $\alpha_{z^{\prime}}^{t}$ that appears in the second bullet of (5-6). The function $\alpha_{z^{\prime}}^{t}$ is defined by an element, $\left.\mathfrak{c}_{z^{\prime}}^{t}\right|_{(w, t)} \in \mathfrak{C}_{1}$. The identification of $\mathfrak{C}_{1}$ with $\mathbb{C}=\operatorname{Sym}^{1}(\mathbb{C})$ identifies $T_{1,0} \mathfrak{C}_{1}$ at $\left.\mathfrak{c}_{z^{\prime}}^{t}\right|_{(w, t)}$ with $\mathbb{C}$. Meanwhile, the bundle $\left.V_{C}\right|_{\left(w, t, z^{\prime}\right)}$ is isomorphic to the normal bundle of $\mathcal{E}$ at $\left(w, t, z^{\prime}\right)$. The latter is trivialized by the exponential map $e_{c}$ using Property 4 in Section 4.a. This identification of $\left.V_{C}\right|_{\left(w, t, z^{\prime}\right)}$ with $\mathbb{C}$ and the identification of $T_{1,0} \mathfrak{C}_{1}$ at $\left.\mathfrak{c}_{z^{\prime}}\right|_{(w, t)}$ with $C$ identifies $\left.V_{C}\right|_{\left(w, t, z^{\prime}\right)}$ with the latter. This understood, the homomorphism $\phi$ in Part 5 of Section 2.a maps the complex line $\left.V_{C}\right|_{\left(w, t, z^{\prime}\right)}$ to $\left.\left(\mathfrak{c}_{\gamma-}^{*} T_{1,0} \mathfrak{C}_{m_{\gamma-}}\right)\right|_{(w, t)}$.

To continue, suppose next that $\mathbb{R} \times \gamma$ is a component of $\Sigma$, and let $q$ denote the corresponding integer. Let $\mathcal{E}$ denote the end $(-\infty,-1] \times \gamma$ of $\Sigma$. The point $(w, t, 0) \in$ $\mathcal{E}$ corresponds to a zero of $\left.\alpha^{\gamma-}\right|_{(w, t)}$ of order $q$ at the origin. This corresponds to the factor $\alpha_{0}^{0}$ that appears in the second point of (5-6). The latter is defined by the symmetric vortex $\mathfrak{c}_{0} \in \mathfrak{C}_{q}$. Note in this regard that $T_{1,0} \mathfrak{C}_{q}$ at $\mathfrak{c}_{0}$ is canonically isomorphic to $\mathbb{C}^{q}$; this isomorphism given by the coordinates in (1-5). Meanwhile, $V_{\mathbb{R} \times \gamma}$ is the product of $\mathbb{R} \times \gamma$ with $\mathbb{C}^{q}$ and so $\left.V_{\mathbb{R} \times \gamma}\right|_{(w, t, 0)}$ is canonically isomorphic to $T_{1,0} \mathfrak{C}_{q}$ at $\mathfrak{c}_{0}$. This understood, the homomorphism $\phi$ from Part 5 of Section 2.a also maps $\left.V_{\mathbb{R} \times \gamma}\right|_{(w, t, 0)}$ to $\mathfrak{c}_{\gamma-}^{*} T_{1,0} \mathfrak{C}_{m_{\gamma}}$.

These homomorphisms define a homomorphism from $\times_{C \in \mathcal{C}} C^{\infty}\left(\left.C\right|_{|w|<R_{*}} ; V_{C}\right)$ to the space $C^{\infty}\left(\left(-R_{*},-R\right) \times S^{1} ; \mathfrak{c}_{\gamma-}^{*} T_{1,0} \mathfrak{C}_{m_{\gamma-}}\right)$. The latter is denoted by $\phi_{\gamma-}$. There is an analogous $\phi_{\gamma+}$ for each $\gamma \in \Xi_{\Sigma+}$ with the same domain and image in the space $C^{\infty}\left(\left(R, R_{*}\right) \times S^{1} ; \mathfrak{c}_{\gamma+}^{*} T_{1,0} \mathfrak{C}_{m_{\gamma+}}\right)$.

Step 4 With the stage now set, a Banach space $\mathcal{K}$ is obtained by completing a set of "smooth" elements with respect to a certain norm. A smooth element $\xi$ can be written as a vector $\xi=\left(\left(\xi_{C}\right)_{C \in \mathcal{C}},\left(\xi_{\gamma_{-}}\right)_{\gamma \in \Xi_{\Sigma_{-}}},\left(\xi_{\gamma+}\right)_{\gamma \in \Xi_{\Sigma+}}\right)$ whose components are characterized as follows:

- Each $C \in \mathcal{C}$ version of $\xi_{C}$ is a smooth section of $V_{C}$ over the $|s| \leq R$ part of $C$.

- The $\gamma \in \Xi_{\Sigma-}$ component $\xi_{\gamma-}$ is a smooth section over $(-\infty,-R] \times S^{1}$ of $\mathfrak{c}_{\gamma-}^{*} T_{1,0} \mathfrak{C}_{m_{\gamma}}$ with compact support and such that $\xi_{\gamma-}=\phi_{\gamma-}\left(\left(\xi_{C}\right)_{C \in \mathcal{C}}\right)$ on $\left(-R_{*},-R\right) \times S^{1}$.

- The $\gamma \in \Xi_{\Sigma+}$ component $\xi_{\gamma+}$ is a smooth section over $[R, \infty) \times S^{1}$ of $\mathfrak{c}_{\gamma+}^{*} T_{1,0} \mathfrak{C}_{m_{\gamma+}}$ with compact support and such that $\xi_{\gamma+}=\phi_{\gamma+}\left(\left(\xi_{C}\right)_{C \in \mathcal{C}}\right)$ on $\left(R, R_{*}\right) \times S^{1}$. 
The set of smooth elements is denoted by $\mathcal{K}_{\text {smooth }}$. The square of the norm that defines the Banach space assigns to any given smooth element $\xi$ the number

$$
\begin{aligned}
\sum_{C \in \mathcal{C}} \|( & \left.-\sum_{\mathcal{E} \in \mathcal{E}_{C}} \chi_{\mathcal{E}}\right) \xi_{C} \|_{\mathcal{K}}^{2} \\
& +\sum_{\gamma \in \Xi_{\Sigma-}}\left\|\chi_{R-}\left(\xi_{\gamma-}-\phi_{\gamma-}\left(\left(\left(1-\sum_{\mathcal{E} \in \mathcal{E}_{C}} \chi_{\mathcal{E}}\right) \xi_{C}\right)_{C \in \mathcal{C}}\right)\right)\right\|_{\mathcal{K}}^{2} \\
& +\sum_{\gamma \in \Xi_{\Sigma+}}\left\|\chi_{R+}\left(\xi_{\gamma+}-\phi_{\gamma+}\left(\left(\left(1-\sum_{\mathcal{E} \in \mathcal{E}_{C}} \chi_{\mathcal{E}}\right) \xi_{C}\right)_{C \in \mathcal{C}}\right)\right)\right\|_{\mathcal{K}}^{2},
\end{aligned}
$$

where the notation is as follows: First, $\chi_{\mathcal{E}}$ is defined just prior to (5-9) and $\chi_{R \pm}$ are defined just prior to (5-7). The left most version of $\|\cdot\|_{\mathcal{K}}$ when $C \in \mathcal{C}$ is not $\mathbb{R}$-invariant is given in (2-27) with $E=N, n=1$ and $\mathfrak{c}$ the section of $\mathfrak{C}_{N 1}$ that assigns the symmetric vortex to each point. If $C$ is $\mathbb{R}$-invariant with associated integer $q$, then the corresponding version of $\|\cdot\|_{\mathcal{K}}$ is given by (2-27) with data $E=C \times \mathbb{C}, n=q$ and $\mathfrak{c}$ the constant section of $\mathfrak{C}_{E, q}$ that assigns to each point the symmetric vortex. Each $\gamma \in \Xi_{\Sigma-}$ version of $\|\cdot\|_{\mathcal{K}}$ is given by (2-27) using $C=(-\infty,-R) \times S^{1}, \mathfrak{c}_{\gamma-}$ in lieu of $\mathfrak{c}$, and $m_{\gamma-}$ in lieu of $n$. The $\gamma \in \Xi_{\Sigma+}$ version has an analogous definition. It follows from (2-4), (2-5), (4-4) and (4-9) that (5-13) defines a bonafide norm. The norm defined by $(5-13)$ is also denoted by $\|\cdot\|_{\mathcal{K}}$.

When $\xi \in \mathcal{K}$, use $\|\xi\|_{\infty}$ to denote the norm that is obtained by replacing each version of $\|\cdot\|_{\mathcal{K}}$ in (5-13) by the corresponding $L^{\infty}$ norm. Likewise, use $\|\xi\|_{\mathcal{K}_{1}^{2}}$ and $\|\xi\|_{\mathcal{K} *}$ to denote the respective norms that are obtained by replacing each version of $\|\cdot\|_{\mathcal{K}}$ in (5-13) by the corresponding versions of (2-27)'s norms $\|\cdot\|_{\mathcal{K}_{1}^{2}}$ and $\|\cdot\|_{\mathcal{K} *}$.

Fix $\varepsilon_{0}>0$ such that the map êxp from Part 8 of Section 2.a embeds the radius $10^{6} \varepsilon_{0}$ ball in each fiber of $T \mathfrak{C}_{m}$ for each $m$ from 1 through the maximum of $\left\{m_{\gamma-}\right\}_{\gamma \in \Xi_{\Sigma-}} \cup$ $\left\{m_{\gamma+}\right\}_{\gamma \in \Xi_{\Sigma+}}$. With $\varepsilon_{0}$ fixed, let $\mathcal{K}_{*}$ denote the completion using the norm $\|\xi\|_{\mathcal{K} *}$ of the subspace in $\mathcal{K}_{\text {smooth }}$ with $\|\xi\|_{\infty}<\varepsilon_{0}$. Note that the function $\xi \rightarrow\|\xi\|_{\infty}$ is continuous on $\mathcal{K}_{*}$ and, of course, bounded by $\varepsilon_{0}$. By construction, the function $\xi \rightarrow\|\xi\|_{\mathcal{K} *}$ is also continuous on $\mathcal{K}_{*}$, but this function has no upper bound on $\mathcal{K}_{*}$. Meanwhile, $\|\cdot\|_{\mathcal{K}}$ need not be finite on any given element in $\mathcal{K}_{*}$.

Step 5 Fix $\xi=\left(\left(\xi_{C}\right)_{C \in \mathcal{C}},\left(\xi_{\gamma-}\right)_{\gamma \in \Xi_{\Sigma_{-}}},\left(\xi_{\gamma+}\right)_{\gamma \in \Xi_{\Sigma+}}\right) \in \mathcal{K}_{*}$. Suppose that $C \in \mathcal{C}$ is not $\mathbb{R}$-invariant, and let $\pi: N \rightarrow C$ denote the projection. Define the pair $\left(\mathbb{A}^{C \xi}, \alpha^{C \xi}\right)$ of connection on $E=\pi^{*} N \rightarrow N$ and section of $E$ over $N$ by using (2-36) with the constant section of $\mathfrak{C}_{N, 1}$ given by the symmetric vortex used to define $(A, \alpha)$, and with $\xi_{C}$ in place of $\zeta_{C}$. Rescale with the map $\hat{r}$ and denote the result as $\left(\mathbb{A}^{C \xi r}, \alpha^{C \xi r}\right)$. The latter defines the pair $\left(\mathbb{A}^{C \xi r}, \psi=\left(\alpha^{C \xi r}, 0\right)\right)$ of connection on $E$ over $U_{C}$, and section of $\mathbb{S}$ over $U_{C}$. Write this pair as $\left(A^{*}, \psi^{*}\right)+\mathfrak{t}^{C \xi}$, where $\mathfrak{t}^{C \xi}$ is a section of $i T^{*} M \oplus \mathbb{S}$ over $U_{C}$. 
Suppose next that $C=\mathbb{R} \times \gamma \in C$ and let $q$ denote the associated integer. With $C$ 's normal bundle trivialized as in Property 5 of Section 4.a, use the constant map from $C$ to the symmetric vortex and $\xi_{C}$ to define $\left(\mathbb{A}^{C \xi}, \alpha^{C \xi}\right)$ on $C \times \mathbb{C}$ as instructed by (2-36). Rescale the result using the map $\hat{r}_{\gamma}$ and again use $\left(\mathbb{A}^{C \xi r}, \alpha^{C \xi r}\right)$ to denote the result. View the appropriate restriction of the pair $\left(\left(\mathbb{A}^{C \xi r}, \psi=\left(\alpha^{C \xi r}, 0\right)\right)\right.$ as a connection on $E$ over $U_{c}$, and section of $S$ over $U_{C}$. Write this pair of connection and section as $\left(A^{*}, \psi^{*}\right)+\mathfrak{t}^{C \xi}$ so as to define $\mathfrak{t}^{C \xi}$ as a section of $i T^{*} M \oplus \mathbb{S}$ over $U_{C}$.

Step 6 Suppose that $\gamma \in \Xi_{\Sigma-}$. The map êxp $\hat{c}_{\mathfrak{c}}$ from the symmetric vortex in $\mathfrak{C}_{m_{\gamma-}}$ gives local coordinates for $\mathfrak{C}_{m_{\gamma-}}$ on a neighborhood of this vortex; thus it identifies $T_{1,0} \mathfrak{C}_{m_{\gamma-}}$ on this neighborhood as a trivial bundle. As the $w \ll-1$ part of $\mathfrak{c}_{\gamma-}$ is very close to the constant map from $S^{1}$ to the symmetric vortex in $\mathfrak{C}_{m_{\gamma-}}$, this local trivialization of $T_{1,0} \mathfrak{C}_{m_{\gamma-}}$ allows $\zeta_{\gamma-}$ to be viewed as a section of $\mathfrak{c}_{\gamma-}^{*} T_{1,0} \mathfrak{C}_{m_{\gamma-}}$ on the $w \ll-1$ part of $\mathbb{R} \times S^{1}$. Extend this section to a smooth section, $\zeta_{*}$, over the whole of $\mathfrak{c}_{\gamma-}^{*} T_{1,0} \mathfrak{C}_{m_{\gamma-}}$ so as to vanish where $w \geq-2 R_{*}$. There exists such an extension which obeys the $\mathbb{R} \times S^{1}$ version of (5-10). Such an extension should be selected.

With $\xi \in \mathcal{K}_{*}$ chosen, define the pair $\left(\mathbb{A}^{\gamma \xi-}, \alpha^{\gamma \xi-}\right)$ of connection on, and section of the product bundle over $(-\infty,-R) \times S^{1} \times \mathbb{C}$ by

(5-14) $\mathbb{A}^{\gamma \xi-}=\theta_{0}+A_{0}^{\gamma-}+A^{\gamma-}+2^{-1 / 2}\left(q_{\xi} d \bar{z}-\bar{q}_{\xi} d z\right) \quad$ and $\quad \alpha^{\gamma \xi-}=\alpha^{\gamma-}+\varsigma_{\xi}$.

The notation used here is as follows: First, $\theta_{0}$ is the flat connection given by the product bundle. Meanwhile, $\left(q_{\xi}, \varsigma_{\xi}\right)$ are defined at each point in $\mathbb{R} \times S^{1}$ by the version of

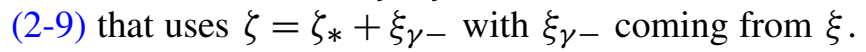

To continue, let $\left(\mathbb{A}^{\gamma \xi-, r}, \alpha^{\gamma \xi-, r}\right)$ denote the pullback of $\left(\mathbb{A}^{\gamma \xi-}, \alpha^{\gamma \xi-}\right)$ via the map $\hat{r}_{\gamma}$. View $\left(\mathbb{A}^{\gamma \xi-, r}, \psi=\left(\alpha^{\gamma \xi-, r}, 0\right)\right)$ as a pair of connection on $E$ over $U_{\gamma-\text { and section }}$ of $S$ over $U_{\gamma-}$. Write the latter as $\left(A^{*}, \psi^{*}\right)+\mathfrak{t}^{\gamma \xi-}$ where $\mathfrak{t}^{\gamma \xi-}$ is a section over $U_{\gamma-}$ of $i T^{*} M \oplus \mathbb{S}$ with zero component in the $E K^{-1}$ summand of $\mathbb{S}$.

Make the analogous construction for those $\gamma \in \Xi \gamma+$ to define from $\xi$ the section $\mathfrak{t}^{\gamma \xi+}$ over $U_{\gamma+}$ of $i T^{*} M \oplus \mathbb{S}$.

Step 7 With $\left(\left(\mathfrak{t}^{C \xi}\right)_{C^{\prime} \in C},\left(\mathfrak{t}^{\gamma \xi-}\right)_{\gamma \in \Xi_{\Sigma-}},\left(\mathfrak{t}^{\gamma \xi+}\right)_{\gamma \in \Xi_{\Sigma+}}\right)$ in hand, define $\left(A^{\xi}, \psi^{\xi}\right)$ to denote the pair of connection on $E$ and section of $S$ given by

$$
\begin{aligned}
\left(A^{*}, \psi^{*}\right) & +\sum_{C \in \mathcal{C}} \chi_{C}\left(1-\sum_{\mathcal{E} \in \mathcal{E}_{C}} \chi_{\mathcal{E}_{C}}\right) \mathfrak{t}^{C \xi} \\
& +\sum_{\mathcal{E} \in \mathcal{E}} \sum_{\gamma \in \Xi_{\Sigma-}} \chi_{\mathcal{E}_{C}} \chi_{\gamma-\mathfrak{t}^{\gamma \xi-}} \\
& +\sum_{\mathcal{E} \in \mathcal{E}} \sum_{\gamma \in \Sigma_{+}} \chi_{\mathcal{E}_{C}} \chi_{\gamma+} \mathfrak{t}^{\gamma \xi+} .
\end{aligned}
$$


Here, $\chi_{E_{C}}$ is defined as follows: Let $C \in \mathcal{C}$ denote the component that contains $\mathcal{E}$. Then $\chi_{\mathcal{E}_{C}}$ is the function on $\mathbb{R} \times M$ with support on $e_{C}\left(\left.N_{4 \Sigma}\right|_{\mathcal{E}}\right)$ where it is given by the rule $\chi_{\mathcal{E}_{C}}=\chi\left(4|s| / \rho_{\Sigma}\right) \chi\left(\left(R_{\mathcal{E}}+3 R-|s|\right) / R\right)$.

\section{5.c Another way to view the construction}

This subsection describes a somewhat different way to view (5-9) and (5-15). What is written in (5-9) and (5-15) interpolate between one construction that takes place on an embedded disk bundle neighborhood of $\Sigma$ and another that takes place on the $|s| \gg R$ part of $\mathbb{R} \times M$ where a given exponential map is many to one. Thus, where two or more points in the large $\pm s$ slices of $\Sigma$ are very close to a single point on an integral curve of $v$.

To say more, fix attention on some $\gamma \in \Xi_{\Sigma-}$. Here is how to think of $\left(A^{\xi}, \psi^{\xi}\right)$ on $U_{\gamma-}$ : Any given constant $(w, t)$ slice has two regions where $A^{\xi}$ is not almost flat and $\psi^{\xi}$ is not almost covariantly constant. The first region consists of the $\rho_{\Sigma}$ neighborhood of the part of $\Sigma \cap U_{\gamma}$ - where $|z|$ is $O(1) r^{-1 / 2+2 \sigma}$ or larger. Here, the pair $\left(A^{\xi}, \psi^{\xi}\right)$ comes from some suitably rescaled $\mathfrak{C}_{1}$ vortex in $\Sigma$ 's normal bundle. The second region lies where $|z| \ll r^{-1 / 2+2 \sigma}$. The $z \rightarrow r_{\gamma}^{1 / 2}$ rescaling of these points in $\Sigma$ define a solution on $\mathbb{C}$ to the vortex equations in (1-4). The inverse scaling of this vortex gives $\left(A^{\xi}, \psi^{\xi}\right)$ where $|z| \ll r^{-1 / 2+2 \sigma}$.

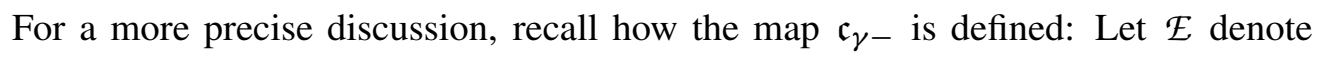
an end from $\mathcal{E}_{\gamma_{-}}$; thus an end of $\Sigma$ in $U_{\gamma-}$. If $\mathcal{E}$ is not part of $\mathbb{R} \times \gamma$, introduce $q_{\mathcal{E}} \in\{1,2, \ldots\}$ to denote the multiplicity of the projection from any given constant $s$ slice of $\mathcal{E}$ to $S^{1}$. It follows from (4-2) that $\mathcal{E}$ appears in $U_{\gamma-}$ as the image of a map, $(w, t) \rightarrow\left(w, t, z_{\mathcal{E}}(w, t)\right)$, of a map from $(-\infty,-R] \times\left(\mathbb{R} / 2 \pi q_{\mathcal{E}} \mathbb{Z}\right)$ to $\mathbb{R} \times S^{1} \times D$. The map $z_{\mathcal{E}}$ is used to define

$$
\left.\mathfrak{Z}_{\mathcal{E}}\right|_{(w, t)}=\left\{z_{\mathcal{E}}(w, t+2 \pi), z_{\mathcal{E}}(w, t+4 \pi), \ldots, z_{\mathcal{E}}\left(w, t+2 \pi q_{\mathcal{E}}\right)\right\} \in \operatorname{Sym}^{q_{\mathcal{E}}}(\mathbb{C}) .
$$

If $\mathcal{E}$ is the $w \ll-1$ part of $\mathbb{R} \times \gamma$, set $q_{\mathcal{E}}$ to equal the integer that is paired with $\mathbb{R} \times \gamma$ as a member of $\Sigma$. Define $\mathfrak{Z}_{\mathcal{E}}$ as above with $z_{\mathcal{E}}$ set to 0 . Introduce the set $\left.\mathfrak{Z}_{\Sigma \gamma-}\right|_{(w, t)}=\left.\bigcup_{\mathcal{E} \in \mathcal{E}_{\gamma-}} \mathfrak{Z}_{\mathcal{E}}\right|_{(w, t)}$ where $\mathcal{E}_{\gamma-}$ denotes the set of ends of $\Sigma$ that lie in $U_{\gamma-}$. The rescaled set $r_{\gamma}^{1 / 2} \mathfrak{Z} \Sigma \gamma-$ defines a map from $(-\infty,-R] \times S^{1}$ to $\operatorname{Sym}^{m_{\gamma-}}(\mathbb{C})$ and as such, a map from $(-\infty,-R] \times S^{1}$ to $\mathfrak{C}_{m_{\gamma-}}$. This last map is $\mathfrak{c}_{\gamma-}$.

With the preceding understood, let $N=N_{\gamma-}$ denote the number of distinct elements in the set $\left\{\lambda_{q_{\mathcal{E}}}: \mathcal{E} \in \mathcal{E}_{\gamma-}\right\}$. Here, $\lambda_{q_{\mathcal{E}}}<0$ is the eigenvalue that appears in $\mathcal{E}$ 's version of (4-2) if $\mathcal{E}$ is not in $\mathbb{R} \times \gamma$. If $\mathcal{E} \subset \mathbb{R} \times \gamma$, then $\lambda_{q_{\mathcal{E}}}$ is the symbol $-\infty$. Partition $\mathcal{E}_{\Sigma \gamma-}$ into $N$ subsets $\mathcal{E}_{1}, \mathcal{E}_{2}, \ldots, \mathcal{E}_{N}$ using the equivalence relation $\mathcal{E} \sim \mathcal{E}^{\prime}$ if and 
only if $\lambda_{q_{\mathcal{E}}}=\lambda_{q_{\mathcal{E}^{\prime}}}$. Use the ordering that is defined by the absolute values of the $\lambda_{q_{\mathcal{E}}}$ to label these partition subsets; thus $\lambda_{q_{\mathcal{E}}}>\lambda_{q_{\mathcal{E}^{\prime}}}$ when $\mathcal{E} \subset \mathcal{E}_{k-1}$ and $\mathcal{E}^{\prime} \subset \mathcal{E}_{k}$. Given $k \in\{1, \ldots, N\}$, define the integer $m_{k}=\sum_{k \leq j \leq N} \sum_{\mathcal{E} \in \mathcal{E}_{j}} q_{\mathcal{E}}$, and then define

$$
\left.\mathfrak{Z}_{k}\right|_{(w, t)}=\bigcup_{k \leq j \leq N}\left(\left.\bigcup_{\mathcal{E} \in \mathcal{E}_{j}} \mathfrak{Z}_{\mathcal{E}}\right|_{(w, t)}\right) \in \operatorname{Sym}^{m_{k}}(\mathbb{C}) .
$$

For example, $\mathfrak{Z}_{1}=\mathfrak{Z}_{\Sigma} \gamma-$. Here is a second example: If $\mathbb{R} \times \gamma \subset \Sigma$ and has assigned integer $q$, then $\left.\mathfrak{Z}_{N}\right|_{(w, t)}$ consists of $q$ copies of the origin in $\mathbb{C}$.

Recall for what follows that $R_{\mathcal{E}}=\frac{1}{4}\left|\lambda_{q_{\mathcal{E}}}\right|^{-1}(1-4 \sigma) \ln$ when $\mathcal{E}$ is not in $\mathbb{R} \times \gamma$. The set $\mathfrak{Z}_{1}$ is of interest where $w$ is less than the $\mathcal{E} \in \mathcal{E}_{1}$ version of $-R_{\mathcal{E}}-R$. Meanwhile, the set $\mathfrak{Z}_{2}$ is of interest where $w$ is greater than the $\mathcal{E} \in \mathcal{E}_{1}$ version of $-R_{\mathcal{E}}-4 R$ but less than the $\mathcal{E} \in \mathcal{E}_{3}$ version of $-R_{\mathcal{E}}-R$. In general, the $1<k \leq N$ version of $\mathfrak{Z}_{k}$ is of interest where $w$ is greater than the $\mathcal{E} \in \mathcal{E}_{k-1}$ version of $-R_{\mathcal{E}}-4 R$ but less than $-R_{\mathcal{E}}-R$ as defined by an end $\mathcal{E} \in \mathcal{E}_{k}$. This $w$ interval of interest for $\mathcal{E}_{k}$ is denoted by $I_{k}$ in what follows.

The rescaled map $r_{\gamma}^{1 / 2} \mathfrak{Z}_{k}$ maps $I_{k} \times S^{1}$ into $\operatorname{Sym}^{m_{k}}(\mathbb{C})$ and thus into $\mathfrak{C}_{m_{k}}$. Use $\mathfrak{c}_{k}$ to denote the latter. With regards to $\mathfrak{c}_{k}$, suppose that $k \neq N$, or if so, that $\mathfrak{Z}_{N} \neq(0, \ldots, 0)$. The fourth point in (4-9) implies that all $\left.z \in r_{\gamma}^{1 / 2} \mathfrak{Z}_{k}\right|_{(w, t)}$ have norm $O\left(r^{-\sigma}\right)$ except those that come from $\mathcal{E}_{k}$. The latter have size $O\left(r^{-\sigma}\right)$ near the left endpoint of $I_{k}$, but size $O\left(r^{\sigma}\right)$ near the right end point.

It follows from (2-4) and (4-9) that what is written in (5-9) on the $|z|<r^{-1 / 2+\sigma}$ part of $I_{k} \times S^{1} \times \mathbb{C}$ is almost entirely determined by the rescaling via the map $\hat{r}_{\gamma}$ of the vortex $\mathfrak{c}_{k}$ except very near to the left endpoint of $I_{k}$ where the ends from $\mathcal{E}_{k-1}$ contribute. Except for very near this left endpoint, the pullback of $\left(A^{*}, \psi^{*}\right)$ to a constant $(w, t) \in I_{k} \times S^{1}$ plane is almost entirely given where $|z|<c_{0} r^{-1 / 2+\sigma}$ by the pullback via $\hat{r}_{\gamma}$ of a suitable lift of $\mathfrak{c}_{k}$ as a connection on and section of the trivial bundle over $\mathbb{C}$. In particular the difference between the two pairs is bounded in a suitable gauge by an inverse power of $r$ when $r$ is large. Meanwhile, $\left(A^{*}, \psi^{*}\right)$ where $|z|$ has size $c_{0} r^{-1 / 2+3 \sigma / 2}$ or greater is very close to what is written in (5-2).

Now consider $\left(A^{\xi}, \psi^{\xi}\right)$. What is written in (2-4) and (2-5) can be used to construct an injective homomorphism $\phi_{k}: \mathfrak{c}_{k}^{*} T_{1,0} \mathfrak{C}_{m_{k}} \rightarrow \mathfrak{c}_{\gamma-}^{*} T_{1,0} \mathfrak{C}_{m_{\gamma-}}$ over $I_{k} \times S^{1}$ that differs from an isometry by less than $c_{0} e^{-r^{\sigma / 4}} \leq c_{0} r^{-10}$. Use $\phi_{k}^{\dagger}$ to denote the adjoint of this homomorphism. The kernel of $\phi_{k}^{\dagger}$ over $I_{k} \times S^{1}$ is isomorphic to the pushforward of $\Sigma$ 's normal bundle by the projection map

$$
\pi:\left(\left.\bigcup_{\mathcal{E} \in \mathcal{E}_{\gamma-}-\mathcal{E}_{k}} \mathcal{E}\right|_{I_{k} \times S^{1}}\right) \rightarrow I_{k} \times S^{1} .
$$

Said differently, there is an isomorphism and near isometry over $I_{k} \times S^{1}$ between the bundles $\mathfrak{c}_{\gamma-}^{*} T_{1,0} \mathfrak{C}_{m_{\gamma-}}$ and $\mathfrak{c}_{k}^{*} T_{1,0} \mathfrak{C}_{m_{k}} \oplus\left(\bigoplus_{\mathcal{E} \in \mathcal{E}_{\gamma-}-\mathcal{E}_{k}} \pi_{*}\left(\left.N\right|_{\mathcal{E}}\right)\right)$. This is to say 
that if $\xi=\left(\left(\xi_{C}\right)_{C \in \mathcal{C}},\left(\xi_{\gamma-}\right)_{\gamma \in \Xi_{\Sigma-}},\left(\xi_{\gamma+}\right)_{\gamma \in \Xi_{\Sigma+}}\right) \in \mathcal{K}$, then $\xi_{\gamma-}$ can be viewed via this isomorphism as pair $\left(\xi_{k}, \pi_{*}\left(\xi_{\Sigma k}\right)\right)$ where the notation is as follows: First, $\xi_{k}$ is a section over $I_{k} \times S^{1}$ of $\mathfrak{c}_{k}^{*} T_{1,0} \mathfrak{C}_{m_{k}}$. Second, $\xi_{\Sigma k}$ is shorthand for the element in $\left.\bigoplus_{\mathcal{E} \in \mathcal{E}_{\gamma_{-}-}-\mathcal{E}_{k}} N\right|_{\mathcal{E}}$ whose component in the $\left.N\right|_{\mathcal{E}}$ summand is the restriction to $\mathcal{E}$ of the relevant $\xi_{C}$.

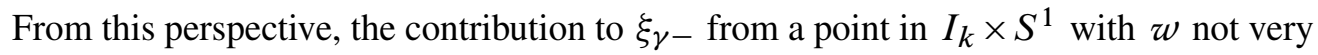
near the left endpoint of $I_{k}$ can be thought of as having two parts: The first, $\xi_{k}$, comes from those components of $\Sigma \cap U_{\gamma}$ - with distance less than or equal to $c_{0} r^{-1 / 2+\sigma}$ from $\mathbb{R} \times \gamma$. The second, $\pi_{*}\left(\xi_{\Sigma k}\right)$, comes from those components of $\Sigma \cap U_{\gamma-}$ with distance at least $c_{0} r^{-1 / 2+\sigma}$ from $\mathbb{R} \times \gamma$. It follows from (2-4) and (2-5) that the $\xi_{k}$ part of $\xi_{\gamma-}$ determines very much the balance of the difference between $\left(A^{\xi}, \psi^{\xi}\right)$ and $\left(A^{*}, \psi^{*}\right)$ on the $|z|<r^{-1 / 2+\sigma}$ part of $U_{\gamma-}$ if $w \in I_{k}$ is not very near the left endpoint. Indeed, the map from $I_{k} \times S^{1}$ to $\mathfrak{C}_{m_{k}}$ given by $\exp _{\mathfrak{c}_{k}}\left(\xi_{k}\right)$ defines a deformation of $\mathfrak{c}_{k}$ and the $\hat{r}_{\gamma}$ rescaling of this deformation is very close to $\left(A^{\xi}, \psi^{\xi}\right)$ where $|z|<r^{-1 / 2+\sigma}$ for the stated values of $w$. Meanwhile, the $\pi_{*}\left(\xi_{\Sigma k}\right)$ part of $\xi_{\gamma-}$ supplies the preponderance of the difference between the pairs $\left(A^{\xi}, \psi^{\xi}\right)$ and $\left(A^{*}, \psi^{*}\right)$ on the part of $U_{\gamma}$ - where $w \in I_{k}$ is not very near the left endpoint and where $|z| \geq c_{0} r^{-1 / 2+3 \sigma / 2}$. But for corrections that are much less than $r^{-1}$, this difference is supported on the $e_{\Sigma}$ image of the bundle $\left.N \rightarrow \bigcup_{\mathcal{E} \in \mathcal{E}_{\gamma-}-\mathcal{E}_{k}} \mathcal{E}\right|_{I_{k} \times S^{1}}$ where it is given by the $\mathfrak{t}^{C \xi}$ sum in (5-11).

\section{5.d The perturbative set up}

The plan now is to look for a solution to the large $r$ version of (1-14) that is gauge equivalent over $\mathbb{R} \times M$ to a pair of connection on, and section of the respective pullbacks of $E$ and $\mathbb{S}$ over $\mathbb{R} \times M$ given by

$$
A=A^{\xi}+(2 r)^{1 / 2}(\phi d s+b) \text { and } \psi=\psi^{\xi}+\eta
$$

for a particular $\xi \in \mathcal{K}_{*} \cap \mathcal{K}$ and section $\mathfrak{b}=(b, \eta, \phi)$ of $i T^{*} M \oplus \mathbb{S} \oplus i \mathbb{R}$ over $\mathbb{R} \times M$. The pair $\xi$ and $\mathfrak{b}$ are chosen so as to have two properties.

Property 1 The following equation is obeyed on $\mathbb{R} \times M$ :

- $\frac{\partial}{\partial s} b+* d b-d \phi-2^{-1 / 2} r^{1 / 2}\left(\psi^{\xi \dagger} \tau \eta+\eta^{\dagger} \tau \psi^{\xi}\right)-2^{-1 / 2} r^{1 / 2} \eta^{\dagger} \tau \eta$ $=-2^{-1 / 2} r^{-1 / 2}\left(\frac{\partial}{\partial s} A^{\xi}+B_{A^{\xi}}-r\left(\psi^{\xi \dagger} \tau \psi^{\xi}-i a\right)-\left(i * d \mu+\frac{1}{2} B_{A_{K}}\right)\right)$,

$$
\begin{array}{ll}
\cdot & \left(\nabla_{A} \xi\right)_{s} \eta+D_{A^{\xi}} \eta+2^{1 / 2} r^{1 / 2}\left(\operatorname{cl}(b) \psi^{\xi}+\phi \psi^{\xi}\right)+2 r^{1 / 2}(\operatorname{cl}(b) \eta+\phi \eta) \\
& =-\left(\nabla_{A^{\xi}}\right)_{s} \psi^{\xi}-D_{A^{\xi}} \psi^{\xi}, \\
- & \frac{\partial}{\partial s} \phi+* d * b-2^{-1 / 2} r^{1 / 2}\left(\eta^{\dagger} \psi^{\xi}-\psi^{\xi \dagger} \eta\right)=0 .
\end{array}
$$


Here, $\left(\nabla_{A^{\xi}}\right)_{S}$ denotes the covariant derivative along the $\mathbb{R}$ factor of $\mathbb{R} \times M$ as defined using the connection $A^{\xi}$. Meanwhile, $d$ here denotes the exterior derivative along the $M$ factor in $\mathbb{R} \times M$.

Property 2 The $s \rightarrow-\infty$ limit of $\mathfrak{b}$ is the solution to (3-4) that is supplied by the $\Theta=\Theta_{-}$version of the constructions in Section 3. Meanwhile, the $s \rightarrow \infty$ limit of $\mathfrak{b}$ is the solution to (3-4) that is supplied by the $\Theta=\Theta_{+}$version of the constructions in Section 3.

Section 6 and Section 7 describe a pair $(\xi, b)$ that has Properties 1 and 2 . Use $(\xi, \mathfrak{b})$ in (5-19) to define the pair $(A, \psi)$. It is argued in Section 7.h that $(A, \psi)$ is smooth. Section 7.h also establishes the following: Let $\mathfrak{c}_{-}$denote the solution to (1-13) that is given in Section 3 using $\Theta=\Theta_{-}$and let $\mathfrak{c}_{+}$denote the solution to (1-13) that is given in Section 3 using $\Theta=\Theta_{+}$. There exists a smooth map $u$ : $\mathbb{R} \times M \rightarrow S^{1}$ such that the assignment $s \rightarrow \mathfrak{d}(s)=\left.\left(A-u^{-1} d u, u \psi\right)\right|_{s}$ is a smooth map from $\mathbb{R}$ into $\operatorname{Conn}(E) \times C^{\infty}(M ; \mathbb{S})$ that defines an instanton solution to (1-14) whose $s \rightarrow-\infty$ limit is $\mathfrak{c}_{-}$and whose $s \rightarrow \infty$ limit is smoothly gauge equivalent to $\mathfrak{c}_{+}$.

\section{The construction of instantons: Part II}

The two properties required of the pair $\xi$ and $\mathfrak{b}$ in Section 5.d are written schematically as

- $\mathfrak{D} \mathfrak{b}+r^{1 / 2} \mathfrak{b} * \mathfrak{b}-\mathfrak{v}=0$.

(6-1) $\quad \lim _{s \rightarrow \pm \infty} \mathfrak{b}=\mathfrak{b}_{ \pm}$where $\mathfrak{b}_{ \pm}$are the respective solutions Section 3 to the $\Theta=\Theta_{ \pm}$versions of (3-5) as defined using $\zeta_{ \pm}$.

Here, $\mathfrak{D}$ and $\mathfrak{v}$ are defined by $\xi$. As done with (3-5), the plan for what follows is to project the top equation in (6-1) onto a certain subspace and then solve the latter with $\xi \in \mathcal{K}_{*} \cap \mathcal{K}$ fixed. The solution, $\mathfrak{b}=\mathfrak{b}(\xi)$, will depend smoothly on $\xi$. The remaining part of the top equation in (6-1) is subsequently viewed as an equation for $\xi$ which is seen to have a unique, small normed solution.

The results of this section are summarized by Proposition 6.4 in Section 6.e.

\section{6.a The projection for (6-1)}

This first subsection is devoted to setting up the projected version of (6-1). There are eight parts to this. In what follows, $\xi$ denotes a given section of $\mathcal{K}_{*}$. 
Part 1 Fix for the moment a connection, $A$, on $E \rightarrow \mathbb{R} \times M$. With $A$ given, define the Hilbert space $\mathbb{H}$ to be the completion of the space of compactly supported sections over $\mathbb{R} \times M$ of $i T^{*} M \oplus \mathbb{S} \oplus i \mathbb{R}$ using the norm whose square is given by

$$
\|\mathfrak{q}\|_{\mathbb{H}}^{2}=\int_{\mathbb{R} \times M}\left|\nabla^{*} \mathfrak{q}\right|^{2}+\frac{1}{4} r \int_{\mathbb{R} \times M}|\mathfrak{q}|^{2},
$$

where the covariant derivative $\nabla^{*}$ acts on sections of $i T^{*} M$ as the Levi-Civita covariant derivative; on sections of $\mathbb{S}$ as the covariant derivative that is defined by Levi-Civita connection and $A_{K}+2 A$; and on any given $i$-valued function as the exterior derivative. Note in what follows that a dimension 4 Sobolev inequality provides an $r$ independent constant $c_{0}$ such that

$$
\|\mathfrak{q}\|_{4} \leq c_{0}\|\mathfrak{q}\|_{\mathbb{H}}
$$

for all $\mathfrak{q} \in \mathbb{H}$. Here, $\|\cdot\|_{4}$ denotes the $\mathbb{R} \times M$ version of the $L^{4}$ norm. Use $L$ to denote the space of $L^{2}$ sections over $\mathbb{R} \times M$ of $i T^{*} M \oplus \mathbb{S} \oplus i \mathbb{R}$.

Suppose next that $\psi$ is a section of $\mathbb{S} \rightarrow \mathbb{R} \times M$. The pair $(A, \psi)$ together define the operator, $\mathfrak{D}$, on $C^{\infty}\left(\mathbb{R} \times M ; i T^{*} M \oplus \mathbb{S} \oplus i \mathbb{R}\right)$ that sends a given section $(b, \eta, \phi)$ to the section with the respective $i T^{*} M, \mathbb{S}$ and $i \mathbb{R}$ components

- $\frac{\partial}{\partial s} b+* d b-d \phi-2^{-1 / 2} r^{1 / 2}\left(\psi^{\dagger} \tau \eta+\eta^{\dagger} \tau \psi\right)$.

- $\left(\nabla_{A}\right)_{s} \eta+D_{A} \eta+2^{1 / 2} r^{1 / 2}(\operatorname{cl}(b) \psi+\phi \psi)$.

- $\frac{\partial}{\partial s} \phi+* d * b-2^{-1 / 2} r^{1 / 2}\left(\eta^{\dagger} \psi-\psi^{\dagger} \eta\right)$.

Here, $\left(\nabla_{A}\right)_{s}$ denotes the covariant derivative along the $\mathbb{R}$ factor of $\mathbb{R} \times M$ as defined using the connection $A$. If the section $\psi$ is bounded, then $\mathfrak{D}$ extends to give a bounded operator from $\mathbb{H}$ to $\mathbb{L}$.

Let $\hat{a}$ denote a bounded section of $i T^{*}(\mathbb{R} \times M)$. Then the $A+\hat{a}$ and $A$ versions of the norm $\|\cdot\|_{\mathbb{H}}$ are commensurate. Thus, the corresponding versions of $\mathbb{H}$ contain the same elements. This has the following consequence; Suppose in addition that $\varsigma$ is a bounded section of $\mathbb{S} \rightarrow \mathbb{R} \times M$. Then the $(A+\hat{a}, \psi+\varsigma)$ version of (6-4) defines a bounded operator from the $A$ version of $\mathbb{H}$ to $\mathbb{L}$.

In what follows, the connection $A^{*}$ from Section 5 is used to define $\mathbb{H}$. The operator $\mathfrak{D}$ in (6-1) is the version of (6-4) that is defined using $\left(A^{\xi}, \psi^{\xi}\right)$ from (5-15).

Part 2 This part with Parts 3 and 4 constitute a digression to describe in more detail the operator $\mathfrak{D}$ as defined using the given element $\xi \in \mathcal{K}_{*}$ to construct $\left(A^{\xi}, \psi^{\xi}\right)$. What follows describes the $\left(A^{\xi}, \psi^{\xi}\right)$ version of $\mathfrak{D}$ on $U_{0}$. To this end, the identification of $\mathbb{S}$ with $\mathbb{S}_{I}$ on $U_{0}$ identifies $\mathfrak{D}$ over $U_{0}$ with the restriction to $U_{0}$ of the version 
of (6-4) on $C^{\infty}\left(\mathbb{R} \times M ; i T^{*} M \oplus \mathbb{S}_{I} \oplus i \mathbb{R}\right)$ that is defined using $\left(A=A_{I}, \psi=\psi_{I}\right)$. The latter operator is denoted in what follows by $\mathfrak{D}_{I}$.

Part 3 Suppose that $C \in \mathcal{C}$ is not $\mathbb{R}$-invariant. This part describes $\mathfrak{D}$ on $U_{C}$. The description starts by saying more about the bundle $i T^{*} M \oplus \mathbb{S} \oplus i \mathbb{R}$ on $U_{C}$. The summand $i\left(T^{*} M \oplus \mathbb{R}\right)$ is identified with $T^{*}(\mathbb{R} \times M)$ and is thus identified via pullback by $e_{C}$ with $T^{*} N$. The latter splits as $\pi^{*}\left(T^{*} C\right) \oplus V_{\theta}$, where $V_{\theta}$, denotes the subspace that annihilates the $\theta$-horizontal vectors in $T N$. Thus, $V_{\theta}$ is canonically isomorphic as a real bundle $\pi^{*}\left(N^{*}\right)$. This splitting is orthogonal along $C$ for the metric that is pulled back by $e_{C}$ from $\mathbb{R} \times M$. The almost complex structure $J$ defines via $e_{C}$ an almost complex structure on $T^{*} N$ over $e_{C}^{-1}\left(U_{C}\right)$. The $(0,1)$ part of this bundle has a $\mathbb{C}$-linear orthogonal splitting along $C$ as $\left.T^{0,1} C \oplus N\right|_{C}$. This understood, an isomorphism between $\left.\left(\pi^{*} T^{0,1} C \oplus \pi^{*} N\right)\right|_{e_{C}^{-1}\left(U_{C}\right)}$ and $\left.T^{0,1}(\mathbb{R} \times M)\right|_{U_{C}}$ is defined as follows: Any given vector $q \in \pi^{*} N$ is mapped to the 1 -form $q\left(\nabla_{\theta} \overline{\mathfrak{s}}+\varsigma\right)$ where $\varsigma$ has the following properties: It vanishes on $C$ and it can be written as $\varsigma_{0} \nabla_{\theta} \mathfrak{s}+\varsigma_{1}$ with $\left|\varsigma_{0}\right| \leq c_{0}|\mathfrak{s}|$ and with $\varsigma_{1}$ differing from a section of $\pi^{*} T C$ by $c_{0}|s|^{2}$. Meanwhile, any given vector, $p$, in $\pi^{*} T^{0,1} C$ is mapped to a vector of the form $p+\mathrm{L}(p)$ where L: $\pi^{*} T^{0,1} C \rightarrow \pi^{*} T^{1,0} C$ vanishes along $C$.

Write the bundle $\mathbb{S}$ as $E \oplus E K^{-1}$. On $U_{C}$, the bundle $E$ is the pull back via $e_{C}$ as $\pi^{*} N$, and the identifications in the preceding paragraph define an isomorphism over $U_{C}$ between $K^{-1}$ and $\pi^{*}\left(N \otimes T^{0,1} C\right)$. Let $I_{\mathbb{C}}$ denote the product complex line bundle over $C$. The preceding identifications of $\mathbb{S}$ and $T^{*} M \oplus \mathbb{R}$ over $U_{C}$ identify the $e_{C}$-pull back of $\left(i T^{*} M \oplus \mathbb{S} \oplus i \mathbb{R}\right)$ with the direct sum $\mathbb{V}_{C 0} \oplus \mathbb{V}_{C 1} \rightarrow C$ where

$$
\mathbb{V}_{C 0}=\pi^{*} N \oplus \pi^{*} N \quad \text { and } \quad \mathbb{V}_{C 1}=\pi^{*}\left(I_{\mathbb{C}} \oplus N^{2}\right) \otimes \pi^{*}\left(T^{0,1} C\right)
$$

Here, the left hand summands from $\mathbb{V}_{C 0}$ and $\mathbb{V}_{C 1}$ make up $e_{C}^{*}\left(i T^{*} M \oplus i \mathbb{R}\right)$; and the right hand summands make up $e_{C}^{*} \mathbb{S}$. Use these same splittings of $\mathbb{V}_{C 0}$ and $\mathbb{V}_{C 1}$ to write a section of $\mathbb{V}_{C 0}$ as $\left(q, \eta_{0}\right)$ and one of $\mathbb{V}_{C 1}$ as $\left(p, \eta_{1}\right)$.

Granted the preceding, the operator $\mathfrak{D}$ on $U_{C}$ can be written as a linear differential operator that takes a section of $\mathbb{V}_{C 0} \oplus \mathbb{V}_{C 1}$ over $e_{C}^{-1}\left(U_{C}\right)$ and spits out a section of the bundle $\left(\mathbb{V}_{C 0} \otimes \pi^{*} T^{0,1} C\right) \oplus\left(\mathbb{V}_{C 1} \otimes \pi^{*} T^{1,0} C\right)$. The operator is defined so as to send a section $\mathfrak{f}=\left(\left(q, \eta_{0}\right),\left(p, \eta_{1}\right)\right)$ to one whose component in $\mathbb{V}_{C 0} \otimes \pi^{*} T^{0,1} C$ can be written as

$$
\begin{array}{ll}
- & 2\left(-\bar{\partial}^{V} p+2^{-1 / 2} r^{1 / 2} \bar{\psi}_{0}^{\xi} \eta_{1}\right)+2 \bar{\partial}_{\theta}^{H} q+\mathfrak{r}_{00}, \\
\text { - } & 2\left(-\partial_{A^{\xi}} \eta_{1}+2^{-1 / 2} r^{1 / 2} \psi_{0}^{\xi} p\right)+2 \bar{\partial}_{A^{\xi}}^{H} \eta_{0}+\mathfrak{r}_{01},
\end{array}
$$


and whose component in $\mathbb{V}_{C 1} \otimes \pi^{*} T^{1,0} C$ can be written as

- $2\left(\partial^{V} q+2^{-1 / 2} r^{1 / 2} \bar{\psi}_{0}^{\xi} \eta_{0}\right)+2 \partial_{\theta}^{H} p+\mathfrak{r}_{10}$.

- $2\left(\bar{\partial}_{A^{\xi}}^{V} \eta_{0}+2^{-1 / 2} r^{1 / 2} \psi_{0}^{\xi} q\right)+2 \partial_{A^{\xi}}^{H} \eta_{1}+\mathfrak{r}_{11}$.

To explain the notation, first, $\psi_{0}^{\xi}$ denotes the component of $\psi^{\xi}$ in the $e_{C}^{*} E=\pi^{*} N$ summand of $e_{C}^{*} \mathbb{S}$. Second, the $\mathfrak{r}_{* *}$ terms all obey $\left|\mathfrak{r}_{* *}\right| \leq c_{0}(|\mathfrak{s}||\nabla \mathfrak{f}|+|\mathfrak{f}|)$ where $\nabla$ denotes the covariant derivative that is defined using the connection $\theta$ for the left most summands in (6-5) while using the connection $\theta$ with $A^{\xi}$ for the right most summands. The operator $\partial^{V}$ and its $A^{\xi}$ analog denote the holomorphic covariant derivative along the fibers of $\pi$ as defined by $\nabla$. Meanwhile, $\partial_{\theta}^{H}$ and its $A^{\xi}$ analog denote the $(1,0)$ horizontal part of $\nabla$. The barred versions with $V$ and $H$ denote the respective vertical and horizontal $(0,1)$ parts of $\nabla$. Here, the notion of $(1,0)$ and $(0,1)$ is defined using $\left.J\right|_{C}$. Note that (6-6) and (6-7) implicitly use the volume form on $C$ to identify $T^{0,1} C \otimes T^{1,0} C$ with the product bundle $I_{\mathbb{C}}$.

Since $\mathfrak{D}$ is defined originally so as to take a section of $i T^{*} M \oplus \mathbb{S} \oplus i \mathbb{R}$ and return a section of this same bundle, a few words of explanation are in order to describe the implicit isomorphism used in (6-6) and (6-7) between the latter bundle and the vector bundle $\left(\mathbb{V}_{C 0} \otimes \pi^{*} T^{0,1} C\right) \oplus\left(\mathbb{V}_{C 1} \otimes \pi^{*} T^{1,0} C\right)$. This implicit isomorphism is constructed as follows: First, use the exterior product with the 1 -form $d s$ to identify $T^{*} M$ with the bundle, $\Lambda^{2+}$, of self-dual 2-forms on $\mathbb{R} \times M$. This identifies $T^{*} M \oplus \mathbb{R}$ with $\Lambda^{2+} \oplus \mathbb{R}$. Meanwhile, let $T^{0,2} \subset \Lambda_{C}^{2+}$ denote the subspace of forms of type $(0,2)$, and let $I_{\mathbb{C}}$ denote the product complex line bundle over $\mathbb{R} \times M$. Then $\Lambda^{2+} \oplus \mathbb{R}$ can be viewed as the underlying real bundle of the $\mathbb{C}^{2}$ bundle $I_{\mathbb{C}} \oplus T^{0,2}$. Here, the $I_{\mathbb{C}}$ summand corresponds to the $\mathbb{R} \omega \oplus \mathbb{R}$ summand in $\Lambda^{2+} \oplus \mathbb{R}$. Given that $T^{0,2}=K^{-1}$, the top line in (6-6) defines a section of $K^{-1}$ over $U_{C}$, thus a section of $T^{0,2}$. Meanwhile, the top line in (6-7) defines a section of the product bundle over $U_{C}$ and so a section of $I_{\mathbb{C}}$. Thus, the top lines in (6-6) and (6-7) together define a section of $\Lambda^{2+} \oplus i \mathbb{R}$ and so account for the $i\left(T^{*} M \oplus \mathbb{R}\right)$ required identification between $\left(\mathbb{V}_{C 0} \otimes \pi^{*} T^{0,1} C\right) \oplus\left(\mathbb{V}_{C 1} \otimes \pi^{*} T^{1,0} C\right)$ and $i T^{*} M \oplus \mathbb{S} \oplus i \mathbb{R}$.

To finish the identification, introduce $\mathbb{S}_{+}$and $\mathbb{S}_{-}$to denote the respective self-dual and anti-self-dual parts of the $\operatorname{Spin}_{\mathbb{C}}$ bundle of spinors over $\mathbb{R} \times M$. Clifford multiplication by self-dual 2 -forms annihilates $\mathbb{S}_{-}$and Clifford multiplication by anti-self-dual 2forms annihilates $\mathbb{S}_{+}$. The $\pm i$ eigenspaces for Clifford multiplication by the self-dual 2-form $\omega=(1 / \sqrt{2})(d s \wedge a+(1 / 2) d a)$ split $\mathbb{S}_{+}$as $E \oplus E K^{-1}$ and so identifies the latter with $\mathbb{S}$. Meanwhile, the restriction of Clifford multiplication by forms in $T^{0,1}(\mathbb{R} \times M)$ to the $E$ summand in $\mathbb{S}_{+}$identifies $\mathbb{S}_{-}$with $T^{0,1}(\mathbb{R} \times M) \otimes E$. At the same time, Clifford multiplication by $d s$ identifies $\mathbb{S}_{+}$with $\mathbb{S}_{-}$and thus $\mathbb{S}_{-}$ 
with $\mathbb{S}$. The upshot of all this is the identification of $T^{0,1}(\mathbb{R} \times M) \otimes E$ with $\mathbb{S}$. Granted the preceding, observe that the bottom lines in (6-6) and (6-7) define a section of $\left(\pi^{*} N \otimes \pi^{*} T^{0,1} C\right) \oplus \pi^{*} N^{2}$. Writing the latter as $\left(\pi^{*} T^{0,1} C \oplus \pi^{*} N\right) \otimes \pi^{*} N$ identifies it with $T^{0,1}(\mathbb{R} \times M) \otimes E$. This how the bottom lines in (6-6) and (6-7) define a section in the $\mathbb{S}$ summand of $i T^{*} M \oplus \mathbb{S} \oplus i \mathbb{R}$.

Part 4 This part describes $\mathfrak{D}$ on $U_{\gamma-}$ when $\gamma \in \Xi_{\Sigma-}$, on $U_{\gamma+}$ when $\gamma \in \Xi_{\Sigma+}$, and on $U_{C}$ when $C=\mathbb{R} \times \gamma$.

With $\gamma$ fixed, reintroduce the vector spaces $\mathbb{V}_{0}$ and $\mathbb{V}_{1}$ that appear in (3-13) and (3-14). The starting point is a rewriting of the operator $\mathfrak{D}$ on the relevant open set as an operator that maps $C^{\infty}\left((-\infty,-R) \times S^{1} \times \mathbb{C} ; \mathbb{V}_{0} \oplus V_{1}\right)$ to itself. To this end, write the components of an element $\mathfrak{f} \in \mathbb{V}_{0} \oplus \mathbb{V}_{1}$ as $\left(\left(q, \eta_{0}\right),\left(p, \eta_{1}\right)\right.$. Then the $\mathbb{V}_{0}$ and $\mathbb{V}_{1}$ components of $\mathfrak{D}$ are given respectively by (6-6) and (6-7) with the following reinterpretation of the notation: First, $\partial^{V}=\left(2 \pi / \ell_{\gamma}\right)^{1 / 2}(\partial / \partial z)$ and $\partial_{\theta}^{H}-(1 / 2)(\partial / \partial w-i(\partial / \partial t))$. Of course, the covariant versions of these operators are similarly reinterpreted, as are their barred counterparts. In this case, the terms designated by $\mathfrak{r}_{* *}$ obey $\left|\mathfrak{r}_{* *}\right| \leq c_{0}(|z||\nabla \mathfrak{f}|+|\mathfrak{f}|)$.

Part 5 Suppose that $C \in \mathcal{C}$ is not $\mathbb{R}$-invariant. Use the component $\xi_{C}$ from $\xi$ to define the pair $\left(\mathbb{A}^{C \xi, r}, \alpha^{C \xi, r}\right)$ as done in Step 5 of Section 5.b. This is a pair that consists of a connection on $\left.\pi^{*} N \rightarrow N\right|_{C}$ and a section of this bundle. Define a rank 1, complex line bundle $K_{C \xi} \rightarrow C$ whose fiber is the span in $L^{2}\left(N ; \mathbb{V}_{C 0} \oplus \mathbb{V}_{C 1}\right)$ of the vector $\left(\left(q, \eta_{0}\right),(0,0)\right)$ where $q=\left(2^{-1 / 2} r^{1 / 2}\left(1-\left|\alpha^{C \xi, r}\right|^{2}\right)\right.$ and $\eta_{0}=\partial_{A}^{V^{C \xi, r}} \alpha^{C \xi, r}$. Here, $\partial_{A} V^{C \xi, r}$ denotes the $(1,0)$ part of the covariant derivative along the fiber of $N$ as defined by the connection $\mathbb{A}^{C \xi, r}$ and the complex structure $\left.J\right|_{C}$.

Now suppose that $C=\mathbb{R} \times \gamma$ is a component of $\Sigma$. Define $\left(\mathbb{A}^{C \xi, r}, \alpha^{C \xi, r}\right)$ on $C \times \mathbb{C}$ as done in Step 5 of Section 5.b. Identify $C$ with $\mathbb{R} \times S^{1}$ using Property 5 of Section 4.a. Let $q_{C}$ be the integer associated to $C$, and let $K_{C \xi} \rightarrow \mathbb{R} \times S^{1}$ be the $q_{C}$-dimensional complex vector bundle whose fiber at $(w, t) \in \mathbb{R} \times S^{1}$ is the subspace in $L^{2}\left(C ; \mathbb{V}_{0} \oplus \mathbb{V}_{1}\right)$ spanned by the elements of the form $\left(\left(q, \eta_{0}\right),(0,0)\right)$ where $\left(q, \eta_{0}\right)$ are such that

$$
\partial q+2^{-1 / 2} r_{\gamma}^{1 / 2} \bar{\alpha} \eta_{0}=0 \text { and } \bar{\partial}_{A} \eta_{0}+2^{-1 / 2} r_{\gamma}^{1 / 2} \alpha q=0,
$$

where $\alpha=\alpha^{C \xi, r}$ and where $A$ is pullback to $(s, t) \times C$ of $\mathbb{A}^{C \xi, r}$.

Fix $\gamma \in \Xi_{\Sigma-}$ and use $\xi_{\gamma-}$ from $\xi$ with $\xi_{\gamma-}$ from $\xi_{-}$to define the pair $\left(\mathbb{A}^{\gamma \xi-, r}, \alpha^{\gamma \xi-, t}\right)$ as done just after (5-14). Let $K_{\gamma \xi-} \rightarrow(-\infty,-R) \times S^{1}$ denote the rank $m_{\gamma-}$ complex vector bundle whose fiber at any given $(w, t) \in(-\infty,-R)$ is the subspace in $L^{2}\left(\mathbb{C} ; \mathbb{V}_{0} \oplus \mathbb{V}_{1}\right)$ spanned by the elements of the form $\left(\left(q, \eta_{0}\right),(0,0)\right)$ where $\left(q, \eta_{0}\right)$ obey the version of (6-8) with $\alpha=\alpha^{\gamma \xi-, r}$ and with $A$ the pullback of $\mathbb{A}^{\gamma \xi-, r}$ to $(s, t) \times \mathbb{C}$. 
An analogous definition assigns a bundle $K_{\gamma \xi+} \rightarrow(R, \infty) \times S^{1}$ to each $\gamma \in \Xi_{\gamma+}$.

Part 6 Define $\mathcal{K}^{2}$ to be the completion of $\mathcal{K}$ using the norm given by the analog of (5-13) that has each version of $\|\cdot\|_{\mathcal{K}}$ replaced by the corresponding $L^{2}$ norm. Fix $\left.\theta=\left(\left(\theta_{C}\right)_{C \in \mathcal{C}},\left(\theta_{\gamma-}\right)_{\gamma \in \Xi_{\Sigma-}},\left(\theta_{\gamma+}\right)_{\gamma \in \Xi_{\Sigma+}}\right)\right) \in \mathcal{K}^{2}$.

Suppose that $C \in \mathcal{C}$ is not $\mathbb{R}$-invariant. The component $\theta_{C}$ is an $L^{2}$ section of $\mathfrak{c}^{*} V_{1,0} \mathfrak{C}_{N, 1}$ over the part of $C$ where $|s| \leq R_{*}$. Here, $\mathfrak{c}$ is the section that sends each point to the symmetric vortex. The component $\xi_{C}$ from $\xi \in \mathcal{K}_{*}$ is also a section of this bundle, but one with small $\|\cdot\|_{\infty}$-norm. Let $\mathfrak{c}_{C \xi}$ denote $\exp _{\mathfrak{c}}\left(\xi_{C}\right)$. The differential of $\exp _{\mathfrak{c}}$ at $\xi_{C}$ identifies $\theta_{C}$ as an $L^{2}$ section of $\mathfrak{c}_{C \xi}^{*} V_{1,0} \mathfrak{C}_{N, 1}$ over the $|s| \leq R_{*}$ part of $C$. Thus, it can be viewed as an $L^{2}$ section of the bundle $K_{C \xi} \rightarrow C$ as defined in Part 5. As such, it defines a section over $\mathbb{C}$ 's component of $e_{C}^{-1}\left(U_{C}\right)$ of $\mathbb{V}_{C 0} \oplus \mathbb{V}_{C 1}$; at any given point, the latter section is a multiple of the section that has $\mathbb{V}_{C 1}$ component zero and $\mathbb{V}_{C 0}$ component given by the pair $\left(2^{-1 / 2} r^{1 / 2}\left(1-\left|\alpha^{C \xi, r}\right|^{2}\right), \partial_{A^{C \xi, r}}^{V^{C \xi, r}}\right)$. Interpret this section of $\mathbb{V}_{C 0} \oplus \mathbb{V}_{C 1}$ defined by $\theta_{C}$ as a section, $\hat{\theta}_{C \xi}$, of $i T^{*} M \oplus \mathbb{S} \oplus i \mathbb{R}$.

Suppose next that $C=\mathbb{R} \times \gamma$ is from $\mathbb{C}$, and let $q$ denote the associated integer. Let $\mathfrak{c}$ denote the constant map from $C$ to the symmetric vortex in $\mathfrak{C}_{q}$. Let $\mathfrak{c}_{C \xi}$ again denote $\exp _{\mathfrak{c}}\left(\xi_{C}\right)$. The differential of $\exp _{\mathfrak{c}}$ at $\xi_{C}$ identifies $\theta_{C}$ as an $L^{2}$ section of $\mathfrak{c}^{*} T_{1,0} \mathfrak{C}_{q}$ and thus an $L^{2}$ section of the bundle $K_{C \xi} \rightarrow C$. The latter can again be interpreted as an $L^{2}$ section, $\hat{\theta}_{C \xi}$, of $i T^{*} M \oplus \mathbb{S} \oplus i \mathbb{R}$ whose restriction to the fiber over any given point in $C$ is a multiple of a solution to the relevant $(A, \alpha)$ version of (6-8).

By the same token, each $\theta_{\gamma-}$ defines an $L^{2}$ section of the corresponding bundle $K_{\gamma \xi-\text {, }}$, thus a map from $(-\infty,-R) \times S^{1} \times \mathbb{C}$ to $\mathbb{V}_{0} \oplus \mathbb{V}_{1}$. Interpret the latter as an $L^{2}$ section,

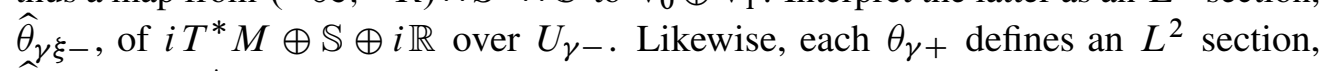
$\hat{\theta}_{\gamma \xi+}$, of $i T^{*} M \oplus \mathbb{S} \oplus i \mathbb{R}$ over $U_{\gamma+}$.

A map $t_{\xi}: \mathcal{K}^{2} \rightarrow \mathbb{L}$ is defined as follows: Let $\theta \in \mathcal{K}^{2}$. Then $t_{\xi}(\theta)$ has support only on $\left(\bigcup_{C \in \mathcal{C}} U_{C}\right) \cup\left(\bigcup_{\gamma \in \Xi_{\Sigma-}} U_{\gamma-}\right) \cup\left(\bigcup_{\gamma \in \Xi_{\Sigma+}} U_{\gamma+}\right)$. Here it is given by

- $t_{\xi}(\theta)=\chi_{C}(1 / \sqrt{\pi}) \hat{\theta}_{C \xi}$ on $U_{C}$.

- $t_{\xi}(\theta)=\left(1-\chi_{\mathcal{E}}\right) \chi_{C}(1 / \sqrt{\pi}) \hat{\theta}_{C \xi}$

$$
+\left(\chi_{C} \chi_{\mathcal{E}}+\left(1-\chi_{C}\right)\right) \chi_{R \pm} \chi_{\gamma \pm}(1 / \sqrt{\pi}) \hat{\theta}_{\gamma \xi \pm}
$$

(6-9) at points in $U_{\gamma \pm}$ with distance $\rho_{\Sigma}$ or less from an end $\mathcal{E}$ in any given component $C$ of $\Sigma$.

- $t_{\xi}(\theta)=\chi_{R \pm} \chi_{\gamma \pm}(1 / \sqrt{\pi}) \hat{\theta}_{\gamma \xi \pm}$ at points in $U_{\gamma \pm}$ with distance $\rho_{\Sigma}$ or more from each component $C$ of $\Sigma$. 
By way of reminder: The functions $\chi_{R \pm}$ and $\chi_{\gamma \pm}$ are defined just prior to (5-7), and $\chi_{\mathcal{E}}$ is defined just prior to (5-9). This map $t_{\xi}$ is a bounded linear injection. Given (2-4), (2-5) and (4-9), the injectivity of $t_{\xi}$ when $r \geq c_{0}$ and $R \geq c_{0}$ can be proved using the perspective taken in Section 5.c. In fact, the map $t_{\xi}$ is nearly isometric.

Let $\Pi_{\xi}$ denote the $L^{2}$ orthogonal projection onto the image of $t_{\xi}$ and let $\mathbb{H} \frac{\perp}{\xi} \subset \mathbb{H}$ denote the kernel of the homomorphism $\Pi_{\xi}: \mathbb{H} \rightarrow \mathbb{L}$. It is a consequence of what is said at the end of Lemma 2.9 that $\Pi_{\xi}$ maps $\mathbb{H}$ to $\mathbb{H}$, and so $\mathbb{H}_{\xi}^{\perp}$ is equal to $\left(1-\Pi_{\xi}\right) \mathbb{H}$.

Part 7 What follows describes a somewhat different version of $\mathcal{K}^{2}$. This version is also a completion of a set of "smooth elements". The typical smooth element is given by the analog of (5-12) where the following modification is understood: If $C \in \mathcal{C}$ is not $\mathbb{R}$-invariant, then $\xi_{C}$ is a section of the bundle $\mathfrak{c}^{*} V_{1,0} \mathfrak{C}_{N, 1} \otimes T^{0,1} C$ over the $|s| \leq R_{*}$ part of $C$. The identifications given by $\phi_{\gamma \pm}$ are as before with it understood that $T^{*} C$ is trivialized over any given end by the pullbacks of $d w$ and $d t$. The norm that defines this space is given by the analog of (5-12) that replaces each $\|\cdot\|_{\mathcal{K}}$ norm with the corresponding $L^{2}$ norm. The resulting Banach space is denoted by $\mathcal{L}^{2}$.

The formula in (6-9) defines a bounded, linear injection from $\mathcal{L}^{2}$ into $\mathbb{L}$. The $\mathcal{L}^{2}$ dual of this injection gives the desired map, $\Pi_{\xi}: \mathbb{L} \rightarrow \mathcal{L}^{2}$. Use $\mathbb{L} \frac{1}{\xi} \subset \mathbb{L}$ to denote the orthogonal complement of the kernel of $\Pi_{\xi}$. This is a closed, linear subspace of $\mathbb{L}$. Although sloppy notation, $\left(1-\Pi_{\xi}\right): \mathbb{L} \rightarrow \mathbb{L}_{\xi} \frac{1}{\xi}$ is used to denote the associated $L^{2}$ orthogonal projection.

Part 8 The promised projection of (6-1) asks for a solution to the equation

$$
\left(1-\Pi_{\xi}\right)\left(\mathfrak{D} \mathfrak{b}+r^{1 / 2} \mathfrak{b} * \mathfrak{b}-r^{-1 / 2} \mathfrak{v}\right)=0
$$

subject to the constraint $\lim _{s \rightarrow \pm \infty} \mathfrak{b}=\mathfrak{b}_{ \pm}$.

\section{6.b Operator norms}

The next lemma is central to the approach taken here to solve (6-10).

Lemma 6.1 Fix $\Sigma$ as described in Section 4.b, but do not assume Constraint 4. There exists a constant $\kappa>1$ with the following significance: Take $r \geq \kappa$. Fix $\xi \in \mathcal{K}_{*}$ with $\|\xi\|_{\infty} \leq \kappa^{-1}$ so as to define $\mathfrak{D}, \mathbb{H}_{\xi}^{\perp}$ and $\mathbb{L}_{\xi}^{\frac{\perp}{\xi}}$. Then

$$
\kappa^{-1}\|\mathfrak{q}\|_{\mathbb{H}} \leq\left\|\left(1-\Pi_{\xi}\right) \mathfrak{D} \mathfrak{q}\right\|_{2} \leq \kappa\|\mathfrak{q}\|_{\mathbb{H}} \quad \text { for all } \mathfrak{q} \in \mathbb{H}_{\xi}^{\perp}
$$

Moreover, if $\xi \in \mathcal{K}_{*}$ has norm $\|\xi\|_{\mathcal{K} *} \leq \kappa^{-1}$ the corresponding $\left(1-\Pi_{\xi}\right) \mathfrak{D}$ maps $\mathbb{H}_{\xi} \frac{\perp}{\xi}$ onto $\mathbb{L} \frac{\perp}{\xi}$. In particular, $\left(1-\Pi_{\xi}\right) \mathfrak{D}: \mathbb{H} \frac{\perp}{\xi} \rightarrow \mathbb{L}_{\xi}^{\frac{\perp}{\xi}}$ is invertible and the norm of its inverse is bounded by $\kappa^{-1}$. 
Proof of Lemma 6.1 The asserted upper bound is straightforward to prove and so omitted. What follows next proves the assertion about lower bound for $\left\|\left(1-\Pi_{\xi}\right) \mathfrak{D q}\right\|_{2}$ when $\mathfrak{q} \in \mathbb{H}_{\xi}^{\perp}$. To start, write $\mathfrak{q}=\mathfrak{q}_{0}+\sum_{C \in \mathcal{C}} \mathfrak{q}_{C}+\sum_{\gamma \in \Xi_{\Sigma-}} \mathfrak{q}_{\gamma-}+\sum_{\gamma \in \Xi_{\Sigma+}} \mathfrak{q}_{\gamma+}$, where

$$
\begin{array}{ll}
\text { - } \mathfrak{q}_{0}=\left(1-\sum_{C \in \mathcal{C}} \chi_{C}\right)\left(1-\chi_{R-} \sum_{\gamma \in \chi_{\Sigma+}} \chi_{\gamma-}-\chi_{R+} \sum_{\gamma \in \Xi_{\Sigma+}} \chi_{\gamma+}\right) \mathfrak{q} . \\
\text { - } \mathfrak{q}_{C}=\chi_{C}\left(1-\chi_{R-} \sum_{\gamma \in \Xi_{\Sigma+}} \chi_{\gamma-}-\chi_{R+} \sum_{\gamma \in \Xi_{\Sigma+}} \chi_{\gamma+}\right) \mathfrak{q} . \\
\text { - } \mathfrak{q}_{\gamma-}=\chi_{\gamma-\chi_{R-} \mathfrak{q} .} \\
\text { - } \mathfrak{q}_{\gamma+}=\chi_{\gamma+\chi_{R+} \mathfrak{q} .}
\end{array}
$$

The section $\mathfrak{q}_{0}$ has support where $\left(A^{\xi}, \psi^{\xi}\right)=\left(A_{I}, \psi_{I}\right)$. This being the case, it follows from these equations and from [7, (5.23)] that there exists $c_{0}>1$ such that if $r>c_{0}$, then

$$
\left\|\mathfrak{D} \mathfrak{q}_{0}\right\|_{2} \geq c_{0}^{-1}\left\|\mathfrak{q}_{0}\right\|_{\mathbb{H}}
$$

But for two items, the arguments that are used to prove the left most inequality in Lemma 3.2 and Lemma 3.3 can be employed here with purely notational changes to find a constant $c_{0} \geq 1$ such that when $r \geq c_{0}$ then

$$
\left\|\left(1-\Pi_{\xi}\right) \mathfrak{D} \mathfrak{q}_{C}\right\|_{2} \geq c_{0}^{-1}\|\mathfrak{q}\|_{\mathbb{H}} \quad \text { and } \quad\left\|\left(1-\Pi_{\xi}\right) \mathfrak{D} \mathfrak{q}_{\gamma \pm}\right\|_{2} \geq c_{0}^{-1}\left\|\mathfrak{q}_{\gamma \pm}\right\|_{\mathbb{H}} .
$$

The first substantive item concerns the reference to Lemma 2.5 in the Lemma 3.2 argument: The reference is replaced in the new argument by a reference to Lemma 2.8. The second item to be noted when applying the arguments for Lemma 3.2 and Lemma 3.3 is the following: Let $C$ denote a component of $\Sigma$ and let $\mathcal{E}$ denote an $s \ll-1$ end of $C$. The constant $s$ slices of $\mathcal{E}$ converge as $s \rightarrow-\infty$ to the Reeb orbit $\gamma_{\mathcal{E}}$. Given (2-4), (2-5), and (4-9), a section over $\mathcal{E}$ of the bundle $K_{C \xi}$ looks like a section over $\left(-R_{*},-R\right) \times S^{1}$ of the bundle $K_{\gamma \xi}$ - when viewed from the perspective of Section 6.c. Conversely, a section of $K_{\gamma \xi-}$ defines a section of $K_{C \xi}$ over the ends of $C$ in $U_{\gamma-}$. There is, of course, a similar remark concerning $C$ 's positive ends.

Granted (6-12) and (6-13), a direct analog of (3-20) implies the lower bound inequality given here.

Consider next the assertion that $\left(1-\Pi_{\xi}\right) \mathfrak{D}$ is onto when $\|\xi\|_{\mathcal{K} *}$ is small. To this end, let $\kappa$ denote the constant that gives the lower bound inequality in the lemma. Now fix $\varepsilon \in\left(0, \frac{1}{2} \kappa^{-1}\right)$ and suppose that $x: \mathbb{H} \rightarrow \mathbb{L}$ is a bounded operator such that $\|\chi \mathfrak{q}\|_{2} \leq \varepsilon\|\mathfrak{q}\|_{\mathbb{H}}$ for all $\mathfrak{q} \in \mathbb{H}$. Then

$$
\left\|\left(1-\Pi_{\xi}\right)(\mathfrak{D}+\chi) \mathfrak{q}\right\|_{2} \geq \frac{1}{2} \kappa^{-1}\|\mathfrak{q}\|_{\mathbb{H}}
$$


for all $\mathfrak{q} \in \mathbb{H}_{\xi}^{\perp}$. Suppose that $\left(1-\Pi_{\xi}\right)(\mathfrak{D}+\chi): \mathbb{H}_{\xi}^{\perp} \rightarrow \mathbb{L}_{\xi}^{\perp}$ is invertible. Then it follows from (6-14) that its inverse has norm bounded by $2 \kappa$. This uniform norm bound implies that the operator $\left(1-\Pi_{\xi}\right) \mathfrak{D}: \mathbb{H}_{\xi}^{\perp} \rightarrow \mathbb{L}_{\xi}^{\perp}$ is invertible if $\varepsilon<(1 / 100) \kappa^{-1}$.

It follows from (2-11) and (2-12) that both $\mathbb{H}_{\xi} \frac{\perp}{\xi}$ and $\mathbb{L}_{\xi} \frac{1}{\xi}$ vary in $\mathbb{H}$ and $\mathbb{L}$ in a uniformly continuous fashion as $\xi$ varies in $\mathcal{K}_{*}$. This understood, the argument from the preceding paragraph implies the following: If the $\xi=0$ version of $\left(1-\Pi_{\xi}\right) \mathfrak{D}$ is invertible as a map from $\mathbb{H}_{\xi=0}^{\perp}$ to $\mathbb{L}_{\xi=0}^{\perp}$, then there exists $\kappa>1$ such that $\left(1-\Pi_{\xi}\right) \mathfrak{D}$ is invertible from $\mathbb{H}_{\xi}^{\perp}$ to $\mathbb{L}_{\xi}^{\frac{1}{\xi}}$ if $\|\xi\|_{\mathcal{K} *} \leq \kappa^{-1}$.

In the case where $\xi=0$, or is smooth, there is a straightforward analog of (2-23) that can be applied here in conjunction with the formula in (6-7) and (6-8) that finds

$$
\left(1-\Pi_{\xi}\right) \mathfrak{D} \Pi_{\xi}=\left(1-\Pi_{\xi}\right) \mathfrak{R} \Pi_{\xi}-\left(1-\Pi_{\xi}\right) x \Pi_{\xi},
$$

where $\|\mathfrak{R q}\|_{2} \leq c\|\mathfrak{q}\|_{2}$ with $c$ a constant, and with $\chi$ a first order operator that obeys

$$
\|\chi \mathfrak{q}\|_{2} \leq c_{0} r^{-1 / 2} \rho_{\Sigma}^{-1}\|\mathfrak{q}\|_{\mathbb{H}}
$$

Indeed, $\chi$ has two contributions. The first comes from the terms that are indicated by $\mathfrak{r}_{* *}$ in (6-6) and (6-7) and the second from the fact that the exponential map $e_{C}$ on any given end of any component $C \subset \Sigma$ does not quite map the fibers of $N_{1} \rightarrow C$ to constant $(s, t)$ slices of $\mathbb{R} \times S^{1} \times C$ unless $C=\mathbb{R} \times \gamma$. In any event, given $\varepsilon>0$, it follows from (6-16) that there exists $\kappa>1$ such that $\|\chi \mathfrak{q}\|_{2} \leq \varepsilon\|\mathfrak{q}\|_{\mathbb{H}}$ when $r \geq \kappa$. In light of the preceding paragraph, it is thus sufficient to prove that $\left(1-\Pi_{\xi=0}\right)(\mathfrak{D}+\chi): \mathbb{H}_{0}^{\perp} \rightarrow \mathbb{L}_{0}^{\perp}$ is invertible when $r$ is large.

To this end, remark that the norm bound in (6-14) implies that $\left(1-\Pi_{0}\right)(\mathfrak{D}+\chi)$ has closed range in $\mathbb{L}_{0}^{\perp}$ and so its cokernel is isomorphic to the kernel of its adjoint. Note that this adjoint is not the formal $L^{2}$ adjoint; it is the adjoint that maps from $\mathbb{L}_{0}^{\perp}$ to $\mathbb{H}_{0}^{\perp}$. The formal $L^{2}$ adjoint of $\left(1-\Pi_{0}\right)(\mathfrak{D}+\chi)$ is $\left(1-\Pi_{0}\right)\left(\mathfrak{D}^{\dagger}+\chi^{\dagger}\right)$ where $\mathfrak{D}^{\dagger}$ and $x^{\dagger}$ denotes the respective formal $L^{2}$ adjoints of $\mathfrak{D}$ and $\chi$, and where $\left(1-\Pi_{0}\right)$ here denotes the $L^{2}$ orthogonal projection onto $H_{0}^{\perp} \subset \mathbb{H}$. The maximal domain of this formal $L^{2}$ adjoint consists of the elements in $\mathbb{L}_{0}^{\perp} \cap \mathbb{H}$.

Suppose for the sake of argument that $\mathfrak{f} \in \mathbb{L}_{0}^{\perp} \cap \mathbb{H}$ and $\mathfrak{f}$ is also in the kernel of the adjoint of $\left(1-\Pi_{0}\right)(\mathfrak{D}+\chi)$. Then $\left(1-\Pi_{0}\right)\left(\mathfrak{D}^{\dagger}+\chi^{\dagger}\right) f=0$. In this case, the argument used above to prove (6-14) can be repeated to prove that $\mathfrak{f}=0$. This understood, it is sufficient now to prove that the kernel of the adjoint of $\left(1-\Pi_{0}\right)(\mathfrak{D}+\chi)$ is in $\mathbb{H}$. To do so, suppose that $z \in \mathbb{H}$. By virtue of (6-15) and (6-16),

$$
\left\|\left(1-\Pi_{0}\right)(\mathfrak{D}+\chi) \Pi_{0 \mathfrak{z}}\right\|_{2} \leq c\left\|_{\mathfrak{z}}\right\|_{2} .
$$


Thus, if $\mathfrak{f} \in \mathbb{L}_{0}^{\perp}$ is in the kernel of the adjoint of $\left(1-\Pi_{0}\right)(\mathfrak{D}+\chi): \mathbb{H}_{0}^{\perp} \rightarrow \mathbb{L}_{0}^{\perp}$, then

$$
\mid\langle\mathfrak{f},(\mathfrak{D}+\chi) \mathfrak{z}\rangle_{2} \leq c\|\mathfrak{f}\|_{2}\left\|_{\mathfrak{z}}\right\|_{2}
$$

for all $\mathfrak{z} \in \mathbb{H}$. This implies that $\mathfrak{f}$ is in the domain of the formal $L^{2}$ adjoint of $\mathfrak{D}+\chi$, and hence $\mathfrak{f} \in \mathbb{H}$. Given what was said earlier, $\mathfrak{f}=0$.

\section{6.c Solving (6-10)}

A solution to (6-10) when $\xi$ is in a small radius ball in $\mathcal{B} \in \mathcal{K}$ is described below. It has the form $\mathfrak{b}=\mathfrak{h}+\mathfrak{q}$ where $\mathfrak{q} \in \mathbb{H} \frac{\perp}{\xi}$ has small norm. Here, $\mathfrak{h}$ serves two purposes:

- It supplies the desired $s \rightarrow \pm \infty$ limits. In particular, $\lim _{s \rightarrow \pm \infty} \mathfrak{h}=\mathfrak{b}_{ \pm}$.

- It facilitates the use of Lemma 6.1.

What follows elaborates on the second point. The plan is to find this particular element $\mathfrak{q} \in \mathbb{H}_{\xi}^{\perp}$ as the fixed point of a map from $\mathbb{H}_{\xi}^{\perp}$ to itself that sends any given $\mathfrak{q}$ to

$$
\mathbb{T}(\mathfrak{q})=\mathbb{D}^{-1}\left(1-\Pi_{\xi}\right)\left(\left(\mathfrak{v}-\mathfrak{v}_{\mathfrak{h}}\right)-r^{1 / 2} \mathfrak{q} * \mathfrak{q}-2 r^{1 / 2} \mathfrak{h} * \mathfrak{q}\right),
$$

where $\mathbb{D}$ is shorthand for $\left(1-\Pi_{\xi}\right) \mathfrak{D}: \mathbb{H}_{\xi} \frac{\perp}{\xi} \rightarrow \mathbb{L}_{\xi}^{\frac{1}{\xi}}$ and where

$$
\mathfrak{v}_{h}=\mathfrak{D h}+r^{1 / 2} \mathfrak{h} * \mathfrak{h} .
$$

Granted Lemma 6.1, then (6-20) gives a well defined self map of $\mathbb{H}_{\xi}^{\perp}$ provided that

- The map $\mathfrak{q} \rightarrow \mathfrak{h} * \mathfrak{q}$ defines a bounded map from $\mathbb{H}_{\xi} \frac{\perp}{\xi}$ to $\mathbb{L}$.

- $\mathfrak{v}-\mathfrak{v}_{\mathfrak{h}} \in \mathbb{L}$.

In what follows, (6-22) will be required to hold such that $\mathfrak{h} *(\cdot)$ : $\mathbb{H}_{\xi} \frac{\perp}{\xi} \rightarrow L$ has small operator norm and such that $\left(1-\Pi_{\xi}\right)\left(\mathfrak{v}-\mathfrak{v}_{\mathfrak{h}}\right)$ has small $L^{2}$ norm. These last two constraints are responsible for certain parts of $\mathfrak{h}$.

To quantify this small norm business, remark that the existence of a fixed point to $\mathbb{T}$ is proved below with the help of the contraction mapping theorem. The use of the latter requires that $\mathbb{T}$ map some ball in $\mathbb{H}_{\xi}^{\perp}$ to itself. Consider, in light of this constraint, the term on the far right in (6-20) that is linear in $\mathfrak{q}$ : According to Lemma 6.1, the $\mathbb{H}$-norm of the contribution to $\mathbb{T}$ from the linear term is no greater than $\kappa r^{1 / 2}\|\mathfrak{h} * \mathfrak{q}\|_{2}$. Thus, the linear contribution to $\mathbb{T}$ is norm decreasing provided that

$$
\|\mathfrak{h} * \mathfrak{q}\|_{2} \leq \frac{1}{4} c_{0}^{-1} r^{-1 / 2}\|\mathfrak{q}\|_{\mathbb{H}} \quad \text { for all } \mathfrak{q} \in \mathbb{H} .
$$

The fact that $\mathbb{T}$ must map a ball to itself also makes demands on the term in (6-20) that is quadratic in $\mathfrak{q}$. As it turns out, these demands can be met when $\left\|\mathfrak{v}-\mathfrak{v}_{\mathfrak{h}}\right\|_{2}$ has 
small $L^{2}$ norm. Indeed, an appeal first to Lemma 6.1 and then to (6-3) finds that

$$
\left\|\mathbb{D}^{-1}\left(1-\Pi_{\xi}\right)\left(r^{1 / 2} \mathfrak{q} * \mathfrak{q}\right)\right\|_{\mathbb{H}} \leq c_{0} r^{1 / 2}\|\mathfrak{q}\|_{4}^{2} \leq c_{0} r^{1 / 2}\|\mathfrak{q}\|_{\mathbb{H}}^{2} .
$$

Granted only (6-23) and (6-24), then $\mathbb{T}$ can map a ball in $\mathbb{H}_{\xi}^{\perp}$ about the origin to itself if the ball has radius less than $\frac{1}{4} c_{0}^{-1} r^{-1 / 2}$. This is insured only if the $\mathfrak{q}$-independent term in (6-20) lies well inside this ball. Given Lemma 6.1, it is in the radius $r^{-1 / 2} \delta$ ball when

$$
\left\|\left(1-\Pi_{\xi}\right)\left(\mathfrak{v}-\mathfrak{v}_{\mathfrak{h}}\right)\right\|_{2} \leq c_{0}^{-1} r^{-1 / 2} \delta .
$$

This last bound quantifies the small $L^{2}$ norm constraint on $\mathfrak{v}-\mathfrak{v}_{\mathfrak{h}}$.

The preceding discussion is summarized by the following lemma.

Lemma 6.2 The constant $\kappa$ from Lemma 6.1 can be chosen so as to guarantee the following: Fix $r \geq \kappa$ and an element $\xi \in \mathcal{K}_{*} \cap \mathcal{K}$ with $\|\xi\|_{\mathcal{K}}<\kappa^{-1}$. Use $\xi$ to define $\mathbb{T}$ as in (6-20) with $\mathfrak{h}$ obeying (6-23) and such that $\left\|\left(1-\Pi_{\xi}\right)\left(\mathfrak{v}-\mathfrak{v}_{\mathfrak{h}}\right)\right\|_{2} \leq \kappa^{-1} r^{-1 / 2}$. Then $\mathbb{T}$ is a contraction mapping on the ball of radius $\kappa^{-1} r^{-1 / 2}$ in $\mathbb{H} \frac{\perp}{\xi}$. Thus, the map $\mathbb{T}$ has a unique fixed point in this ball, and the latter has $\mathbb{H}$-norm bounded by $\kappa\left\|\left(1-\Pi_{\xi}\right)\left(\mathfrak{v}-\mathfrak{v}_{\mathfrak{h}}\right)\right\|_{2}$.

Proof of Lemma 6.2 Given what is said above, it suffices to verify that $\kappa$ can be found so as to guarantee that $\mathbb{T}$ is a contraction on the radius $\kappa^{-1} r^{-1 / 2}$ ball in $\mathbb{H} \frac{1}{\xi}$. Granted (6-23), this follows from the bilinear version of (6-24):

$$
\begin{aligned}
\| \mathbb{D}^{-1}\left(1-\Pi_{\xi}\right)\left(r^{1 / 2} \mathfrak{q}^{\prime} * \mathfrak{q}^{\prime}\right. & \left.-r^{1 / 2} \mathfrak{q} * \mathfrak{q}\right) \|_{\mathbb{H}} \\
& \leq c_{0} r^{1 / 2}\left\|\mathfrak{q}^{\prime}-\mathfrak{q}\right\|_{4}\left(\left\|\mathfrak{q}^{\prime}\right\|_{4}+\|\mathfrak{q}\|_{4}\right) \\
& \leq c_{0} r^{1 / 2}\left\|\mathfrak{q}^{\prime}-\mathfrak{q}\right\|_{\mathbb{H}}\left(\left\|\mathfrak{q}^{\prime}\right\|_{\mathbb{H}}+\left\|\mathfrak{q}^{\prime}\right\|_{\mathbb{H}}\right) .
\end{aligned}
$$

\section{6.d The description of $\mathfrak{h}$}

The lemma that follows asserts that a section $\mathfrak{h}$ of $i T^{*} M \oplus \mathbb{S} \oplus i \mathbb{R}$ can be found with all of the desired properties. The lemma refers to the norm $\|\cdot\|_{\mathcal{K}_{1}^{2}}$ that is obtained by replacing each version of $\|\cdot\|_{\mathcal{K}}$ in (6-20) by the norm defined by the top line of (2-27).

Lemma 6.3 The constant $\kappa$ that appears in Lemma 6.1 can be chosen so as to guarantee the following: Fix $r \geq \kappa$ and suppose that $\xi \in \mathcal{K}_{*}$ has $\|\xi\|_{\infty} \leq \kappa^{-1}$. Then there exists $\mathfrak{h}$ such that

- $\lim _{s \rightarrow \pm \infty} \mathfrak{h}=\mathfrak{b}_{ \pm}$.

- $\|\mathfrak{h} * \mathfrak{q}\|_{2} \leq r^{-9 / 16}\|\mathfrak{q}\|_{\mathbb{H}}$ for all $\mathfrak{q} \in \mathbb{H}$.

Moreover, if $\|\xi\|_{\mathcal{K}_{1}^{2}}$ is finite, then

- $\left\|\left(1-\Pi_{\xi}\right)\left(\mathfrak{v}-\mathfrak{v}_{\mathfrak{h}}\right)\right\|_{2} \leq \kappa r^{-1 / 2}\left(r^{-1 / 2+8 \sigma}+\|\xi\|_{\mathcal{K}_{1}^{2}}\right)$. 
Proof of Lemma 6.3 The proof that follows has six parts.

Part 1 This part of the proof addresses the $|s| \rightarrow \infty$ limits of $\mathfrak{h}$. To this end, reintroduce $R_{*}$ from (4-8) and introduce the functions $\chi_{-}=\chi\left(s+R_{*}\right)$ and $\chi_{+}=\chi\left(s-R_{*}\right)$. The required behavior as $|s| \rightarrow \infty$ is met if $\mathfrak{h}$ has the form

$$
\mathfrak{h}=\chi_{-} \mathfrak{b}_{-}+\chi_{+} \mathfrak{b}_{+}+\mathfrak{h}_{1},
$$

where $\mathfrak{h}_{1}$ at this point is constrained only by the requirement that its norm should limit to zero as $|s| \rightarrow \infty$. With (6-23) in mind, note that

$$
\left\|\mathfrak{b}_{ \pm} * \mathfrak{q}\right\|_{2} \leq c_{0} r^{-3 / 4}\|\mathfrak{q}\|_{\mathbb{H}}
$$

for all $\mathfrak{q} \in \mathbb{H}^{\perp}$. Indeed, Lemma 3.10 with (3-8) implies that the $L^{4}$ norms on $M$ of $\mathfrak{b}_{+}$and $\mathfrak{b}_{-}$are bounded by $c_{0} r^{-3 / 4}$. Meanwhile, the latter bound on each constant $s$ slice of $\mathbb{R} \times M$ implies the bound that is asserted by (6-28). Lemma 3.10 also implies that

$$
r^{1 / 2}\left\|\left|d \chi_{-}\right|\left|\mathfrak{b}_{-}\right|\right\|_{2}+r\left\|\chi_{-}\left(1-\chi_{-}\right) \mathfrak{b}_{-} * \mathfrak{b}_{-}\right\|_{2} \leq c_{0} r^{-1 / 2} .
$$

A similar inequality holds using $\chi_{+}$and $\mathfrak{b}_{+}$. These inequalities have the following pleasant consequence: Neither the $\chi_{-} \mathfrak{b}_{-}$nor the $\chi_{+} \mathfrak{b}_{+}$contribution to $\mathfrak{h}$ will foul the second and third requirements stated by the lemma if $r$ is sufficiently large.

Part 2 As it happens, $\mathfrak{h}_{1}$ can not, in general, be taken equal to zero. To elaborate, remark that what is written as $\mathfrak{v}$ in (6-10) has a component from the term $(i * d \mu+$ $\left.\frac{1}{2} B_{A_{K}}\right)$ on the right hand side of the top equation in (5-20). With $\mathfrak{h}_{1}=0$, this term contributes something of size $O\left(R_{*}\right) r^{-1 / 2}$ to the $L^{2}$ norm of $\mathfrak{v}-\mathfrak{v}_{\mathfrak{h}}$. This understood, let $\mathfrak{b}_{0}$ denote the small solution to (3-34) and write

$$
\mathfrak{h}=\chi_{-} \mathfrak{b}_{-}+\chi_{+} \mathfrak{b}_{+}+\chi_{0} \mathfrak{b}_{0}+\mathfrak{h}_{2},
$$

where

$$
\chi_{0}=\left(1-\chi_{-}\right)\left(1-\chi_{+}\right)\left(1-\sum_{C \in \mathcal{C}} \chi_{C}\right)\left(1-\chi_{R-} \sum_{\gamma \in \Xi_{\Sigma-}} \chi_{\gamma-}-\chi_{R+} \sum_{\gamma \in \Xi_{\Sigma+}} \chi_{\gamma+}\right) .
$$

Note that (6-28) holds with $\mathfrak{h}_{0}$ replacing $\mathfrak{h}_{ \pm}$and for the same reason: The $\mathbb{H}$ norm of $\mathfrak{b}_{0}$ on $M$ is no greater than $c_{0} r^{-1 / 2}$ and its $L^{\infty}$ norm is no greater than $c_{0} r^{-1}$. These facts about $\mathfrak{b}_{0}$ imply that

$$
\begin{array}{ll}
\text { - } & r^{1 / 2}\left\|\left|d \chi_{0}\right|\left|\mathfrak{b}_{0}\right|\right\|_{2} \leq c_{0} R_{*}^{1 / 2} r^{-1 / 2} \leq c_{0} r^{-1 / 2+8 \sigma} . \\
\text { - } & r\left\|\chi_{0}\left(1-\chi_{0}\right) \mathfrak{b}_{0} * \mathfrak{b}_{0}\right\|_{2} \leq c_{0} r^{-1+8 \sigma} .
\end{array}
$$

- $r \chi\left\|\chi_{ \pm} \chi_{0} \mathfrak{b}_{0} * \mathfrak{b}_{ \pm}\right\|_{2} \leq c_{0} r^{-1+8 \sigma}$. 
By construction, the $\mathfrak{b}_{0}$ contribution to $\mathfrak{h}$ takes care of the contribution to $\mathfrak{v}$ from the part of $r^{-1 / 2}\left(i * d \mu+\frac{1}{2} B_{A_{K}}\right)$ that lies where $|s| \leq R_{*}$ and has distance $\rho_{*}$ or more from $\Sigma$ in that the $L^{2}$ norm of $\left(\mathfrak{v}-\mathfrak{v}_{\mathfrak{h}}\right)$ from this part of $\mathbb{R} \times M$ is bounded by $c_{0} r^{-1+8 \sigma}$. Meanwhile, the rest of the contribution of $r^{-1 / 2}\left(i * d \mu+\frac{1}{2} B_{A_{K}}\right)$ to $\mathfrak{v}-\mathfrak{v}_{\mathfrak{h}}$ is supported at points with distance $c_{0} \rho_{*}$ or less from the $|s|<4 R_{*}$ part of $\Sigma$. Given that the volume of this region is bounded by $c_{0} \rho_{*}^{2} \mathrm{lnr}$, it follows that the $L^{2}$ norm of this contribution to $\mathfrak{v}-\mathfrak{v}_{\mathfrak{h}}$ is bounded by $c_{0} r^{-1+8 \sigma}$ also.

Part 3 It is not possible to take $\mathfrak{h}_{2}=0$ in (6-30) because of contributions to $\mathfrak{v}$ from points with distance $\rho_{*}$ or less from $\Sigma$. In particular, there are relatively large contributions to $\mathfrak{v}$ from each $C \in \mathcal{C}$ version of $U_{C}$ that come from $\left(\mathbb{A}^{C, r}, \alpha^{C, r}\right)$ and $\mathfrak{t}^{C \xi}$. To say more about these contribution, suppose first that $C$ is not $\mathbb{R}$-invariant. Use $\left(x_{\xi}, \iota_{\xi}\right)$ to denote the version of (2-37) that is defined using $\zeta=\xi_{C}$ and using the section of $\mathfrak{C}_{N, 1} \rightarrow C$ that assigns the symmetric vortex to each point for $\mathfrak{c}$. (Note that (2-33)'s pair $(x, \iota)$ is zero in this case.) Let $\left(x_{\xi}^{0,1}, \iota_{\xi}^{0,1}\right)$ denote the part of $\left(x_{\xi}, \iota_{\xi}\right)$ that involves $\pi^{*} T^{0,1} C$. Both $x_{\xi}^{0,1}$ and $\iota_{\xi}^{0,1}$ are sections over $N$ of $\pi^{*}\left(N \otimes T^{0,1} C\right)$. Introduce $\left(x_{\xi, r}^{0,1}, \iota_{\xi, r}^{0,1}\right)$ to denote the pullback via $\hat{r}$ of $\left(x_{\xi}^{0,1}, \iota_{\xi}^{0,1}\right)$.

As in Part 3 of Section 6.a, identify the bundle $i T^{*} M \oplus \mathbb{S} \oplus i \mathbb{R}$ over $U_{C}$ with the bundle $\left(\mathbb{V}_{C 0} \oplus \mathbb{V}_{C 1}\right) \otimes \pi^{*} T^{0,1} C$. The parts of $\mathfrak{v}$ not previously discussed can be written on $U_{c}$ using a calculation like that in Section 2 of the article $\mathrm{Gr} \Rightarrow \mathrm{SW}$ from [5]. In particular, they can be written as $\left.\mathfrak{v}_{C \xi}+\right] m f z$ where $\mathfrak{v}_{C \xi} \in \mathbb{V}_{C 0} \otimes \pi^{*} T^{0,1} C$ and $\mathfrak{z}$ obey

$$
\begin{aligned}
& \text { - } \mathfrak{v}_{C \xi}=\left(x_{\xi, r}^{0,1}-r^{1 / 2} 2^{-1 / 2}\left(v_{C} \mathfrak{s}+\mu_{C} \overline{\mathfrak{s}}\right)\left(1-\left|\alpha^{C, r}\right|^{2}\right)\right. \text {, } \\
& \left.\iota_{\xi, r}^{0,1}-\left(v_{C} \mathfrak{s}+\mu_{C} \overline{\mathfrak{s}}\right) \partial_{A^{C, r}}^{V} \alpha^{C, r}\right) .
\end{aligned}
$$

- $|\mathfrak{z}| \leq c_{0} r^{-1 / 2}\left(1+\left|\nabla \xi_{C}\right|+\left|\xi_{C}\right|\right) e^{-\sqrt{r}|s| / c_{0}}$.

Here, $A^{C, r}$ is the pullback of $\mathbb{A}^{C, r}$ to the fibers of $N$. The norm and covariant derivative of $\xi_{C}$ that appear in (6-32) are defined by viewing $\xi_{C}$ as a section over $C$ of the pullback of the bundle $V_{1,0} \mathfrak{C}_{N, 1} \rightarrow \mathfrak{C}_{N, 1}$ via the section that assigns the symmetric vortex to each point.

Suppose next that $C=\mathbb{R} \times \gamma$ is a component of $\Sigma$ with associated integer $\mathfrak{q}$. Then the parts of $\mathfrak{v}$ on $U_{C}$ not already accounted for can also be written as $\mathfrak{v}_{C \xi}+\mathfrak{z}$ with $|\mathfrak{z}|$ satisfying the inequality in the second bullet of (6-32) and with $\mathfrak{v}_{C \xi}$ viewed as a map to the summand $\mathbb{V}_{0} \subset \mathbb{V}_{0} \oplus \mathbb{V}_{1}$ that is given by the following modification to what appears in the top bullet of (6-32): First, $r_{\gamma}$ replaces $r$, the coordinate $z$ replaces $\mathfrak{s}$, and $\gamma$ 's version of $(\nu, \mu)$ replaces the pair $\left(\nu_{C}, \mu_{C}\right)$. Second, $\left(A^{C, r}, \alpha^{C, r}\right)$ are defined in Step 2 of Part 2 in Section 5.a from the map c: $C \rightarrow \mathfrak{C}_{q}$ that assigns the symmetric 
vortex to each point. Third, $\left(x_{\xi, r}^{0,1}, \iota_{\xi, r}^{0,1}\right)$ are defined using the data supplied by $\xi_{C}$. Indeed, given the identification $\left.E\right|_{C}$ with $C \times \mathbb{C}$ as described in Step 2 of Part 2 in Section 5.a, there is a corresponding version of $\left(x_{\xi}, \iota_{\xi}\right)$, this as defined by (2-37) using $\xi_{C}$ for $\xi$ and using the constant map from $C$ to $\mathfrak{C}_{q}$ that assigns the symmetric vortex to each point for $\mathfrak{c}$. (Note that $(x, \iota)=0$ in this case as well.) Introduce $\left(x_{\xi}^{0,1}, \iota_{\xi}^{0,1}\right)$ to denote the part of $\left(x_{\xi}, \iota_{\xi}\right)$ that involves $\pi^{*} T^{0,1} C$. Both $x_{\xi}^{0,1}$ and $\iota_{\xi}^{0,1}$ map $\mathbb{R} \times S^{1} \times \mathbb{C}$ to $\mathbb{C}$. Now use $\left(x_{\xi, r}^{0,1}, \iota_{\xi, r}^{0,1}\right)$ to denote $\hat{r}_{\gamma}^{*}\left(x_{\xi}^{0,1}, \iota_{\xi}^{0,1}\right)$.

The $L^{2}$ norm of $\mathfrak{v}_{C \xi}$ over $U_{C}$ has size $O\left(\left(\sup _{\mathcal{E} \in \mathcal{E}} R_{\mathcal{E}}\right)^{1 / 2}\right) r^{-1 / 2}$. This is trouble for the lemma's third item. Meanwhile, the $L^{2}$ norm of $r^{-1 / 2}\left(1+\left|\nabla \xi_{C}\right|+\left|\xi_{C}\right|\right) e^{-\sqrt{r}|\mathfrak{s}| / 2}$ over any given component of $U_{C}$ is no greater than $c_{0} r^{-1}\left(\left(\sup _{\mathcal{E} \in \mathcal{E}} R_{\mathcal{E}}\right)^{1 / 2}+\|\xi\|_{\mathcal{K}_{1}^{2}}\right.$; thus the contribution of $\mathfrak{z}$ to the $L^{2}$ norm $\mathfrak{v}$ over $U_{C}$ does not foul the third item in the lemma.

What follows describes how to construct $\mathfrak{h}_{2}$ so that $\mathfrak{h}$ has a term which cancels most of the $\mathfrak{v}_{C \xi}$ contribution to $\mathfrak{v}$. The construction involves a section, $\mathfrak{b}_{C}$, of the bundle $i T^{*} M \oplus \mathbb{S} \oplus i \mathbb{R}$ over $U_{C}$. Suppose that $C \in \mathcal{C}$ is not $\mathbb{R}$-invariant. Then $\mathfrak{b}_{C}$ is given as a section, $\left(0, \mathfrak{p}_{C}\right)$ of $\mathbb{V}_{C 0} \oplus \mathbb{V}_{C 1}$. If $C=\mathbb{R} \times \gamma$, view a section of $i T^{*} M \oplus \mathbb{S} \oplus i \mathbb{R}$ over $U_{C}$ as a map to $\mathbb{V}_{0} \oplus \mathbb{V}_{1}$ and the map $\mathfrak{b}_{C}$ again has the form $\left(0, \mathfrak{p}_{C}\right)$.

If $C$ is not $\mathbb{R}$-invariant, $\mathfrak{p}_{C}$ is defined on the whole of each fiber of $N$ over $C \cap U_{C}$ as the unique section that obeys on each fiber the equation

$$
2 \vartheta_{C, r}^{\dagger} \mathfrak{p}_{C}-\left(1-\Pi^{0}\right) \mathfrak{v}_{C \xi=0}=0,
$$

where the notation is as follows: First, the operator $\vartheta_{C, r}^{\dagger}$ maps sections of $\mathbb{V}_{C 1}$ to those of $\mathbb{V}_{C 0} \otimes \pi^{*} T^{0,1} C$ so as to send any given section $\left(p, \eta_{1}\right)$ of $\mathbb{V}_{C 1}$ to that of $\mathbb{V}_{C 0} \otimes \pi^{*} T^{0,1} C$ with components

$$
-\bar{\partial}^{V} p+2^{-1 / 2} r^{1 / 2} \bar{\alpha}^{C, r} \eta_{1} \text { and }-\partial_{A}^{V, r} \eta_{1}+2^{-1 / 2} r^{1 / 2} \alpha^{C, r} p .
$$

Second, $\Pi^{0}$ denotes the $L^{2}$ orthogonal projection along each fiber of $N$ to the $L^{2}$ kernel of the adjoint, $\vartheta_{C, r}$. Note in this regard that the kernel of $\vartheta_{C, r}$ is spanned on each fiber by the pair $\left(\mathfrak{q}, \eta_{0}\right)=\left(2^{-1 / 2} r^{1 / 2}\left(1-\left|\alpha^{C, r}\right|^{2}\right), \partial_{A^{C, r} \alpha^{C, r}}\right)$.

If $C=\mathbb{R} \times \gamma$, then $\mathfrak{p}_{C}$ is defined on the whole of $\mathbb{C}$ over each point in $C \cap U_{C}$. It is defined on each such copy of $\mathbb{C}$ as the unique solution to the analog of (6-33) that takes $\vartheta_{C, r}^{\dagger}$ to be the operator on $C^{\infty}(\mathbb{C} ; \mathbb{C} \oplus \mathbb{C})$ that is given by the version of (6-34) that uses $\left(2 \pi / \ell_{\gamma}\right)^{1 / 2}(d / d z)$ in lieu of $\partial^{V}$ and $r_{\gamma}$ in lieu of $r$.

In either case, the existence of $\mathfrak{p}_{C}$ follows using (2-7) to solve (6-33) fiberwise along $C$. Moreover, (2-1), (2-2) and (2-7) imply that

$$
\left|\mathfrak{p}_{c}\right|+\left|\nabla^{H} \mathfrak{p}_{C}\right|+r^{-1 / 2}\left|\nabla^{V} \mathfrak{p}_{C}\right| \leq c_{0} r^{-1 / 2} e^{-\sqrt{r}|\mathfrak{s}| / c_{0}},
$$


where $\nabla^{H}$ is the horizontal part of the covariant derivative as defined by $A^{C, r}$ and $\nabla^{V}$ the vertical part.

With $\mathfrak{p}_{C}$ and thus $\mathfrak{b}_{C}$ in hand, write

$$
\mathfrak{h}=\chi_{-} \mathfrak{b}_{-}+\chi_{+} \mathfrak{b}_{+}+\chi_{0} \mathfrak{b}_{0}+\left(\sum_{C \in \mathcal{C}} \chi_{C} \Pi_{\mathcal{E} \in \mathcal{E}_{C}}\left(1-\chi_{\mathcal{E}}\right)\right) \mathfrak{b}_{C}+\mathfrak{h}_{3}
$$

With $\mathfrak{h}_{3}$ zero on $U_{C}$, (6-33) and (6-35) have the following consequence: The $L^{2}$ norm on $U_{C}$ of $\mathfrak{v}-\mathfrak{v}_{\mathfrak{h}}$ is now bounded by $c_{0} r^{-1} \sup _{\mathcal{E} \in \mathcal{E}} R_{\mathcal{E}}$ when $\xi=0$. Take $\mathfrak{h}$ as in (6-36) with $\mathfrak{h}_{3}$ zero on $U_{C}$. If $\xi \neq 0$, then the $L^{2}$ norm of $\mathfrak{v}-\mathfrak{v}_{\mathfrak{h}}$ over $U_{C}$ is bounded by

$$
c_{0}\left(r^{-1} \sup _{\mathcal{E} \in \mathcal{E}} R_{\mathcal{E}}+r^{-1 / 2}\|\xi\|_{\mathcal{K}_{1}^{2}}\right) \leq c_{0}\left(r^{1+8 \sigma}+r^{-1 / 2}\|\xi\|_{\mathcal{K}_{1}^{2}}\right) .
$$

This follows using (2-7) with (2-11), (2-12) and (2-38).

Note that (6-35) also implies that $\left\|\mathfrak{b}_{c} * \mathfrak{q}\right\|_{2} \leq c_{0} r^{-1 / 2}\|\mathfrak{q}\|_{2} \leq c_{0} r^{-1}\|\mathfrak{q}\|_{\mathbb{H}}$ when $\mathfrak{q} \in H$. Thus, the contribution to $\mathfrak{h}$ from $\mathfrak{b}_{C}$ does not cause problems with regards to the second item in the lemma if $r$ is large.

Part 4 It is not in general possible to take $\mathfrak{h}_{3}=0$ in (6-36) because (6-32) has analogs in each $U_{\gamma \pm}$. To say more about this, focus attention on some $\gamma \in \Xi_{\Sigma-}$. There are three regions in $\mathbb{R} \times S^{1} \times \mathbb{C}$ to consider with regards to the $L^{2}$ norm of $\mathfrak{v}$. These are denoted in what follows by $X_{1}, X_{2}$ and $X_{3}$. The first region, $X_{1}$, consists of the points in $U_{\gamma-\subset \mathbb{R}} \times S^{1} \times \mathbb{C}$ where $w \leq-R_{*}$ and $|z| \leq 4 \rho_{*}$. The second region is the complement in $U_{\gamma}$ - of the first region and the third. What follows describes the third region, $X_{3}$ : This is the subset of $U_{\gamma}$ - with the following property: The coordinates $(w, t, z)$ are such that $z$ has distance $\rho_{\Sigma}$ or less from the constant $(w, t)$ slice of an end $\mathcal{E} \subset \Sigma \cap U_{\gamma-}$. Meanwhile, the coordinate $w$ obeys $w>-R_{\mathcal{E}}-8 R$.

Here is a summary of the story for $X_{1}$ : What is written as $\mathfrak{v}$ in (6-1) and (6-10) is all but cancelled by the contribution of $\chi_{-} \mathfrak{b}_{-}$to $\mathfrak{v}_{\mathfrak{h}}$. Indeed, it follows from Constraint 2 in Section 4.b that there is a positive constant, $\lambda \geq c_{0}^{-1}$ with the following significance: Suppose that $\mathfrak{h}_{3}=0$ at points in $U_{\gamma}$ where $w \leq-R_{*}$. Then $\mathfrak{v}-\mathfrak{v}_{\mathfrak{h}}$ at any given $(w, t, z) \in X_{1}$ is bounded by

$$
c_{0}\left(r^{-2} e^{\lambda w}+\left(\left|\nabla \xi_{\gamma-}\right|+\left|\xi_{\gamma-}\right|\right)\right) e^{-\sqrt{r}|z| / c_{0}} .
$$

Here, and in what follows, the covariant derivative and the norm for $\xi_{\gamma}$ - are defined by viewing the latter as a section of the pullback of $T_{1,0} \mathfrak{C}_{m_{\gamma}}$

Given the preceding, it follows using (4-9) that the contribution to the $L^{2}$ norm of $\mathfrak{v}-\mathfrak{v}_{\mathfrak{h}}$ from $X_{1} \subset U_{\gamma-}$ is bounded by $c_{0} r^{-1 / 2}\left(r^{-1 / 2}+\|\xi\|_{\mathcal{K}_{1}^{2}}\right)$. 
Part 5 This part considers the $L_{2}$ norm of $\mathfrak{v}$ in $X_{2}$. There is, first of all, a contribution to this norm from the term $i r^{-1 / 2}\left(* d \mu+\frac{1}{2} B_{A_{K}}\right)$. The $L^{2}$ norm of this contribution on $X_{2}$ is bounded by $c_{0} \rho_{*} r^{-1 / 2}\left(R_{*}-R\right)^{1 / 2}$. In particular, it is too small to bother the third item of the lemma given (4-8). The size of this contribution is bounded by $c_{0} r^{-1+4 \sigma}$.

There is also a contribution to the $L^{2}$ norm of $\mathfrak{v}$ on $X_{2}$ that comes directly from $\left(\mathbb{A}^{\gamma \xi-, r}, \alpha^{\gamma \xi^{-,}, r}\right)$, this the analog of (6-32). This pair is defined in the paragraph that follows (5-14). This contribution can be written as $\left(\mathfrak{v}_{\gamma \xi-}+\mathfrak{z}\right)$. Here, $\mathfrak{v}_{\gamma \xi-}$ on $X_{2}$ maps to $\mathbb{V}_{0}$ and has the form

$$
\mathfrak{v}_{\gamma \xi-}=\left(x_{\xi, r}^{0,1}, \iota_{\xi, r}^{0,1}\right)-(v z+\mu \bar{z})\left(2^{-1 / 2} r_{\gamma}^{1 / 2}\left(1-\left|\alpha^{\gamma \xi, r}\right|^{2}\right), \partial_{A^{\nu \xi-, r}} \alpha^{\gamma \xi-, r}\right),
$$

where the notation is as follows: First, $\left(x_{\xi, r}^{0,1}, \iota_{\xi, r}^{0,1}\right)$ are defined in terms of $\left(\mathbb{A}^{\gamma \xi-}, \alpha^{\gamma \xi-}\right)$ with the latter given in (5-14). To elaborate, introduce the $\left(\mathbb{A}^{\gamma \xi-}, \alpha^{\gamma \xi-}\right)$ versions of what is written in (2-37) and let $\left(x_{\xi}^{0,1}, \iota_{\xi}^{0,1}\right)$ denote the resulting expression. View $\left(x_{\xi}^{0,1}, \iota_{\xi}^{0,1}\right)$ as functions on $(-\infty,-R) \times S^{1} \times \mathbb{C}$. Then $\left(x_{\xi, r}^{0,1}, \iota_{\xi, r}^{0,1}\right)$ designates the pullback of $\left(x_{\xi}^{0,1}, \iota_{\xi}^{0,1}\right)$ via $\hat{r}_{\gamma}$. Second, the pair $\left(A^{\gamma \xi-, r}, \alpha^{\gamma \xi-, r}\right)$ is defined in the paragraph that follows (5-14). Third, $\partial_{A^{\gamma \xi, r}}$ denotes the covariant version of $\left(2 \pi / \ell_{\gamma}\right)^{1 / 2}(\partial / \partial z)$.

What is written as $\mathfrak{z}$ accounts for the various cut-off functions and for the fact that the metric from $\mathbb{R} \times M$ on $U_{\gamma-}$ has $z$ and $t$ dependence. In any event,

$$
|\mathfrak{z}| \leq c_{0} r_{\gamma}^{-1 / 2}\left(1+r|z|^{2}\right)\left(1+\left|\nabla \xi_{\gamma-\mid}\right|+\left|\xi_{\gamma-}\right|\right) \sum_{z^{\prime} \in \mathfrak{Z}_{\gamma-\mid(w, t)}} e^{-\sqrt{r_{\gamma}}\left|z-z^{\prime}\right| / c_{0}} .
$$

The contribution to the $L^{2}$ norm on $X_{2}$ of $\mathfrak{v}$ from $\mathfrak{z}$ does not foul the third item in the lemma because the $L^{2}$ norm on $X_{2}$ of what is written in (6-40) is bounded by

$$
c_{0}\left(r^{-1}+\rho_{*}^{2}\right)\left(R_{*}^{1 / 2}+\|\xi\|_{\mathcal{K}_{1}^{2}}\right) \leq c_{0} r^{-1+8 \sigma}\left(1+\|\xi\|_{\mathcal{K}_{1}^{2}}\right)
$$

On the other hand, the $L^{2}$ norm on $X_{2}$ of $\left(1-\Pi_{\xi}\right) \mathfrak{v}_{\gamma \xi-}$ is $O\left(\rho_{*} R_{*}^{1 / 2}\right)$. This relatively large norm comes from the right most terms in (6-39). Indeed, the $L^{2}$ norm on $X_{2}$ of the term $\left(1-\Pi_{\xi}\right)\left(x_{\xi, r}^{0,1}, \iota_{\xi, r}^{0,1}\right)$ is bounded by $c_{0}\left(1+\|\xi\|_{\mathcal{K}_{1}^{2}}\right) \rho_{*} R_{*}^{1 / 2}$ because the $\xi=0$ version of $\left(x_{\xi, r}^{0,1}, l_{\xi, r}^{0,1}\right)$ is annihilated by the $\xi=0$ version of $\left(1-\Pi_{\xi}\right)$ modulo terms whose $L^{2}$ norm on each constant $(w, t)$ slice in $X_{2}$ is bounded by $c_{0} \rho_{*}$. This understood, let $\mathfrak{v}_{\xi}^{\prime}$ denote the troublesome term, $\mathfrak{v}_{\xi}^{\prime}=\mathfrak{v}_{\gamma \xi-}-\left(x_{\xi, r}^{0,1}, \iota_{\xi, r}^{0,1}\right)$.

What is written as $\mathfrak{h}_{3}$ in (6-36) supplies a term in $\mathfrak{v}_{h}$ that cancels the dominant part of $\left(1-\Pi_{\xi}\right) \mathfrak{v}_{\xi}^{\prime}$. This term in $\mathfrak{h}_{3}$ is defined as follows: Introduce $\vartheta_{\gamma \xi-, r}$ to denote the operator on each constant $(w, t)$ slice of $\left(-R_{*},-R\right) \times S^{1} \times \mathbb{C}$ that is defined by what is written in (6-7). View this operator on each constant $(w, t)$ slice as an operator from $C^{\infty}\left(\mathbb{C} ; \mathbb{V}_{1}\right)$ to $C^{\infty}\left(\mathbb{C} ; \mathbb{V}_{0}\right)$. 
The identities in (2-7) can be used to prove the following: There exists a smooth map $\mathfrak{p}_{\xi}:\left(-R_{*},-R\right) \times S^{1} \times \mathbb{C} \rightarrow \mathbb{V}_{1}$ that obeys $2 \vartheta_{\gamma \xi-, r}^{\dagger} \mathfrak{p}_{\xi}-\left(1-\Pi_{\xi}^{0}\right) \mathfrak{v}_{\xi}^{\prime}=0$ on every constant $(w, t)$ slice of $\left(-R_{*},-R\right) \times S^{1} \times \mathbb{C}$. Here, $\Pi_{\xi}^{0}$ denotes the $L^{2}$-orthogonal projection on each constant $(w, t)$ slice to the $L^{2}$ kernel of $\vartheta_{\gamma \xi-, r}$. Equations (2-1), (2-2) and (2-11), (2-12) with (2-7) imply that this map $\mathfrak{p}_{\xi}$ obeys

$$
\begin{array}{ll}
\text { - } & \left|\mathfrak{p}_{\xi}\right|+r^{-1 / 2}\left|\nabla^{v} \mathfrak{p}_{\xi}\right| \leq c_{\Sigma} \rho_{*}\left(1+\left|\xi_{\gamma-}\right|\right) \sum_{z^{\prime} \in Z_{\gamma-} \mid(w, t)} e^{-\sqrt{r_{\gamma}}\left|z-z^{\prime}\right| / c_{0}} . \\
\text { - }\left|\nabla^{H} \mathfrak{p}_{\xi}\right| \leq c_{\Sigma} \rho_{*}\left(1+\left|\nabla \xi_{\gamma-}\right|+\left|\xi_{\gamma-}\right|\right) \sum_{\left.z^{\prime} \in Z_{\gamma-}\right|_{(w, t)}} e^{-\sqrt{r_{\gamma}}\left|z-z^{\prime}\right| / c_{0}} .
\end{array}
$$

Here $\nabla^{H}$ denotes the $d w$ and $d t$ parts of the covariant derivative on maps to $\mathbb{V}_{1}=\mathbb{C} \oplus \mathbb{C}$ that act as the usual derivative on the first factor and as the derivative that is defined by the connection $\mathbb{A}^{\gamma-, r}$ on the second factor.

With $\mathfrak{p}_{\xi}$ understood, introduce the section $\mathfrak{b}_{\gamma-}$ of $i T^{*} M \oplus \mathbb{S} \oplus i \mathbb{R}$ over the $w>-R_{*}$ part of $U_{\gamma-}$ that is defined by the map from $\left(-R_{*},-R\right) \times S^{1} \times \mathbb{C}$ to $\mathbb{V}_{0} \oplus \mathbb{V}_{1}$ given by the pair $\left(0, \mathfrak{p}_{\xi}\right)$. The contribution to $\mathfrak{h}_{3}$ from $\gamma \in \Xi_{\Sigma-}$ given by

$$
\left(1-\chi_{-}\right) \chi_{R-} \chi_{\gamma-} \mathfrak{b}_{\gamma-} .
$$

With $\mathfrak{h}_{3}$ defined by (6-43) over $X_{2}$, the $L^{2}$ norm of $\left(1-\Pi_{\xi}\right)\left(\mathfrak{v}-\mathfrak{v}_{\mathfrak{h}}\right)$ over $X_{2}$ is bounded by what is written on the right hand side of (6-41). In addition, $\left\|\mathfrak{b}_{\gamma-*} * \mathfrak{q}\right\|_{2} \leq$ $c_{0} \rho_{*}\|\mathfrak{q}\|_{2} \leq c_{\Sigma} r^{-1+3 \sigma}\|\mathfrak{q}\|_{\mathbb{H}}$ and so the contribution from (6-43) to $\mathfrak{h}$ does not cause problems with regards to the second item in the lemma when $r$ is large.

Part 6 Let $\mathcal{E}$ denote an end that intersects $U_{\gamma}$ of a component $C \subset \Sigma$. Set $X_{\mathcal{E}}$ to denote the component of $X_{3}$ where the coordinates $(w, t, z)$ are such that $z$ has distance $\rho_{\Sigma}$ or less from the constant $(w, t)$ slice of $\mathcal{E}$ and the coordinate $w$ obeys $w>-R_{\mathcal{E}}-8 R$. Extend $\mathfrak{h}_{3}$ to $X_{\mathcal{E}}$ as

$$
\left(\chi_{C} \chi_{\mathcal{E}}+\left(1-\chi_{C}\right) \chi_{R-} \chi_{\gamma-}\right) \mathfrak{b}_{\gamma-} .
$$

Consider now the $L^{2}$ norm of $\mathfrak{v}-\mathfrak{v}_{\mathfrak{h}}$ on $X_{\mathcal{E}}$. To this end, consider first the case where $\xi=0$. In this case, $\left(A^{\xi}, \psi^{\xi}\right)$ is given by $\left(A^{*}, \psi^{*}\right)$ with the latter defined on $X_{\mathcal{E}}$ in (5-9). An evident new issue here concerns the fact that $\left(\mathbb{A}^{\mathcal{E}}, \alpha^{\mathcal{E}}\right)$ is not identical in $X_{\mathcal{E}}$ to $\left(\mathbb{A}^{\gamma, r}, \alpha^{\gamma, r}\right)$ when $C$ is not $\mathbb{R}$-invariant. Even so, they almost agree. To quantify this, focus on a disk in $\mathcal{E}$ where the coordinates $(w, t)$ are single valued. Coordinates for the fiber of $N_{4 C}$ over this disk are written as $(w, t, \eta)$. Here, the bundle $N$ is trivialized by the restriction along $C$ of the vector field $\partial / \partial z$. Then $\left(\mathbb{A}^{\mathcal{E}}, \alpha^{\mathcal{E}}\right)$ can be written using these coordinates and the trivialization of $N$ as

$$
\mathbb{A}^{\mathcal{E}}=\theta_{0}+u^{\prime} r(\eta d \bar{\eta}-\bar{\eta} d \eta)+\left(u^{\prime} r|\eta|^{2}-\frac{1}{2}\right)(\overline{\mathfrak{w}}-\mathfrak{w}) \quad \text { and } \quad \alpha^{\mathcal{E}}=r^{1 / 2} e^{-u} \eta
$$


where the notation is as follows: First, $\theta_{0}$ is the product connection for this trivialization. Meanwhile, $\mathfrak{w}$ has only $d s$ and $d t$ components and its coefficients depend only on $w$ and $t$. Moreover $|\mathfrak{w}| \leq c_{\Sigma}$. The 1 -form $\mathfrak{w}$ comes from writing $\nabla_{\theta} \eta=d \eta+\mathfrak{w} \eta$. Finally, $u$ and $u^{\prime}$ are defined in terms of a certain real valued function, $\hat{u}$, on $[0, \infty)$; at any given $(w, t, \eta)$, they have respective values $\widehat{u}\left(r|\eta|^{2}\right)$ and $((d / d y) \hat{u})\left(r|\eta|^{2}\right)$. Note that $\widehat{u}(y)=\frac{1}{2} \ln y+O\left(e^{-y / 2}\right)$ at points $y \geq 1$.

In order to compare (6-45) with $\left(A^{\gamma, r}, \alpha^{\gamma, r}\right)$, it is necessary to pull back the latter via the map that is depicted in (4-4). To this end, use (2-31) to write

$$
\begin{aligned}
& A^{\gamma, r}=\theta_{0}+A_{0}+\left.\mathfrak{u}^{\prime}\right|_{(z-w)} r((z-w) d \bar{z}-(\bar{z}-\bar{w}) d z)+r \\
& \alpha^{\gamma, r}=r^{1 / 2} e^{-\mathfrak{u}}(z-w)+r^{\prime},
\end{aligned}
$$

where the notation here is as follows: First, $w=w(w, t)$ gives the $z$-coordinate of the constant $(w, t)$ slice of the disk in $\Sigma$ under consideration. Second, $\mathfrak{u}=\widehat{u}\left(r|z-w|^{2}\right)$ and $\mathfrak{u}^{\prime}=((d / d y) \widehat{u})\left(r|z-w|^{2}\right)$. Third, both $r$ and $r^{\prime}$ have absolute values bounded by $c_{\Sigma} e^{-\sqrt{r} \rho_{\Sigma} / 2}$. Meanwhile, it follows from (2-3) and (2-5) that $A_{0}$ here is

$$
A_{0}=-\left.\mathfrak{u}^{\prime}\right|_{(z-w)} r((z-w) d \bar{w}-(\bar{z}-\bar{w}) d w)+\mathfrak{r}^{\prime \prime},
$$

where $\left|\mathfrak{r}^{\prime \prime}\right| \leq e^{-\sqrt{r} \rho_{\Sigma} / 2}$ also. Write the pullback of $\mathbb{A}^{\gamma, r}$ as $\theta_{0}+g d \bar{\eta}-\bar{g} d \eta+\overline{\mathfrak{z}}-\mathfrak{z}$ where $\mathfrak{z}$ has only $d s$ and $d t$ components. Granted (6-46) and (6-47), it follows that

$$
\text { - }\left|g-u^{\prime} r \eta\right| \leq c_{0} r^{1 / 2}|z|^{2} e^{-\sqrt{r}|\eta| / c_{0}} \text {. }
$$

$$
\text { - }|\mathfrak{z}| \leq c_{0} e^{-\sqrt{r}|\eta| / c_{0}} \text {. }
$$

- $\left|\alpha^{\gamma, r}-\alpha^{\mathcal{E}}\right| \leq c_{0}|z|^{2} e^{-\sqrt{r}|\eta| / c_{0}}$.

To see what this implies, use $\mathfrak{v}_{\mathcal{E}}$ to denote the version of $\mathfrak{v}$ on $X_{\mathcal{E}}$ that would result were $\left(A^{\xi}, \psi^{\xi}\right)$ defined by taking $\xi=0$ and the $\chi_{\mathcal{E}}=0$ in (5-9). Set $v_{\gamma-}$ to denote the version of $\mathfrak{v}$ that would result were $\left(A^{\xi}, \psi^{\xi}\right)$ defined by taking $\xi=0$ and $\chi_{E}=1$ in (5-9). By virtue of (6-48),

$$
\left|\mathfrak{v}-\left(1-\chi_{\mathcal{E}}\right) \mathfrak{v}_{\mathcal{E}}-\chi_{\mathcal{E}} \mathfrak{v}_{\gamma-}\right| \leq c_{0} r^{-1 / 2}\left(1+r|z|^{4}\right) e^{-\sqrt{r}|\eta| / c_{0}} .
$$

This last inequality is derived with the help of the following identity: Suppose that pairs of numbers $\left(b_{\mathcal{E}}, b_{\gamma_{-}}\right)$and $\left(c_{\mathcal{E}}, c_{\gamma_{-}}\right)$are given and a number $\chi_{\mathcal{E}}$. Then

$$
\begin{aligned}
\left(\chi_{\mathcal{E}} b_{\gamma-}\right. & \left.+\left(1-\chi_{\mathcal{E}}\right) b_{\mathcal{E}}\right)\left(\chi_{\mathcal{E}} c_{\gamma-}+\left(1-\chi_{\mathcal{E}}\right) c_{\mathcal{E}}\right) \\
& =\left(1-\chi_{\mathcal{E}}\right) b_{\mathcal{E}} c_{\mathcal{E}}+\chi_{\mathcal{E}} b_{\gamma-} c_{\gamma-}-\chi_{\mathcal{E}}\left(1-\chi_{\mathcal{E}}\right)\left(b_{\mathcal{E}}-b_{\gamma-}\right)\left(c_{\mathcal{E}}-c_{\gamma-}\right) .
\end{aligned}
$$

Given (6-49), it then follows from (6-35), (6-37) and (6-42) that the $L^{2}$ norm on $X_{\mathcal{E}}$ of $\left(1-\Pi_{\xi}\right)\left(\mathfrak{v}-\mathfrak{v}_{\mathfrak{h}}\right)$ on $X_{\mathcal{E}}$ is no greater than what is written in (6-41) with $\xi=0$. 
In the case when $\xi \neq 0$, the almost identical arguments prove that the $L^{2}$ norm of $\left(1-\Pi_{\xi}\right)\left(\mathfrak{v}-\mathfrak{v}_{\mathfrak{h}}\right)$ is also bounded by what is written in (6-41).

If $\mathbb{R} \times \gamma \subset \Sigma$ and $\mathcal{E}$ is its $w \ll-1$ part, it is also the case that $\left(1-\Pi_{\xi}\right)\left(\mathfrak{v}-\mathfrak{v}_{\mathfrak{h}}\right)$ has its $L^{2}$ norm bounded by what is written in (6-41). The argument for this is much like that just given and left to the reader.

\section{6.e Summary}

The proposition that follows summarizes Lemmas 6.2 and 6.3, and then says a bit more. To set the stage, suppose that some small $v>0$ has been given for use in (2-27) and (2-29). Let $f \in C^{\infty}\left(M ; i T^{*} M \oplus \mathbb{S} \oplus i \mathbb{R}\right)$ denote an element with compact support. Define $\|\mathfrak{f}\|_{*}$ by setting

$$
\|\mathfrak{f}\|_{*}^{2}=\sup _{p \in \mathbb{R} \times M} \sup _{\rho \in(0,1)} \rho^{-v} \int_{\operatorname{dist}(p, \cdot)<\rho}\left(\left|\nabla^{*} \mathfrak{f}\right|^{2}+\rho^{-2}|\mathfrak{f}|^{2}\right) .
$$

Here, as before, $\nabla^{*}$ is the Levi-Civita covariant derivative on sections of $i T^{*} M$, the covariant derivative that is defined by $A^{*}$ on the sections of $\mathbb{S}$, and the exterior derivative on the sections of $i \mathbb{R}$.

Proposition 6.4 Fix $\Sigma$ as in Section 4.b, but do not require Constraint 4. The constant $\kappa$ in Lemmas 6.1-6.3 can be chosen so as to guarantee the following: Fix $r \geq \kappa$ and let $\mathcal{B} \subset \mathcal{K}$ denote the ball of radius $\kappa^{-1}$. Then $\mathcal{B} \subset \mathcal{K}_{*}$. Given $\xi \in \mathcal{B}$, define $\mathfrak{h}=\mathfrak{h}(\xi)$ as instructed by Section 6.d. There exists a unique $\mathfrak{q}=\mathfrak{q}(\xi) \in \mathbb{H} \frac{\perp}{\xi}$ such that

- $\mathfrak{b}=\mathfrak{h}+\mathfrak{q}$ obeys $(6-10)$.

- $\|\mathfrak{q}\|_{\mathbb{H}} \leq r^{-1 / 2} \kappa^{-1}$.

Moreover,

- $\|\mathfrak{q}\|_{\mathbb{H}} \leq \kappa r^{-1 / 2}\left(r^{-1 / 2+8 \sigma}+\|\xi\|_{\mathcal{K}_{1}^{2}}\right.$.

- The assignment $\xi \rightarrow \mathfrak{q}(\xi)$ defines a smooth map from $\mathcal{B}$ to $\mathbb{H}$ and the $\|\cdot\|_{\mathbb{H}}$ norm of the directional derivative of the map $\xi \rightarrow \mathfrak{q}(\xi)$ at any $\xi \in \mathcal{B}$ along any given vector $\xi^{\prime}$ is bounded by $\kappa r^{-1 / 2}\left\|\xi^{\prime}\right\|_{\mathcal{K}}$.

Finally,

- $\|\mathfrak{q}\|_{*} \leq \kappa r^{-1 / 2}\left(r^{-1 / 2+8 \sigma}+\|\xi\|_{\mathcal{K}}\right)$.

- The $\|\cdot\|_{*}$ norm of the directional derivative of the map $\xi \rightarrow \mathfrak{q}(\xi)$ at any $\xi \in \mathcal{B}$ along any given vector $\xi^{\prime}$ is bounded by $\kappa r^{-1 / 2}\left\|\xi^{\prime}\right\|_{\mathcal{K}}$. 
Proof of Proposition 6.4 The existence of $\mathfrak{q}$ and the first three items follow directly from Lemma 6.2 and Lemma 6.3. The fact that the assignment $\xi \rightarrow \mathfrak{q}(\xi)$ is smooth as a map from $\mathcal{B}$ to $\mathbb{H}$ is a standard consequence of the contraction mapping construction given the manner in which $\left(A^{\xi}, \psi^{\xi}\right)$ varies with $\xi$, and given (2-11) and (2-12) to describe the variation of the projection $\Pi_{\xi}$.

To say something about the derivative of this map, fix $\xi \in \mathcal{B}$ and let $\xi^{\prime}$ denote an element in $\mathcal{K}$ with norm 1 . Use $\mathfrak{q}^{\prime}$ to denote the directional derivative of $\mathfrak{q}$ in the direction $\xi^{\prime}$. Write $\mathfrak{q}^{\prime}=\left(1-\Pi_{\xi}\right) \mathfrak{q}^{\prime}+\Pi_{\xi} \mathfrak{q}^{\prime}$. Note that $\Pi_{\xi} \mathfrak{q}^{\prime}=-\Pi_{\xi}^{\prime} \mathfrak{q}$ where $\Pi_{\xi}^{\prime}$ denotes the derivative of the projection in the direction of $\xi^{\prime}$. Use $\mathfrak{q}^{\perp}$ to denote $\left(1-\Pi_{\xi}\right) \mathfrak{q}^{\prime}$.

Properties of $\Pi_{\xi}^{\prime}$ can be deduced using (2-11) and (2-12). In particular, the third bullet in Proposition 6.4 guarantees $\left\|\Pi_{\xi}^{\prime} \mathfrak{q}\right\|_{\mathbb{H}} \leq c_{0} r^{-1 / 2}\left(r^{-1 / 2+8 \sigma}+\|\xi\|_{\mathcal{K}}\right)$. To obtain a bound on the norm of $\mathfrak{q}^{\perp}$, note that the latter obeys an equation that has the schematic form

$$
\left(1-\Pi_{\xi}\right) \mathfrak{D} \mathfrak{q}^{\perp}+\left(1-\Pi_{\xi}\right)\left(2 r^{1 / 2} \mathfrak{q} * \mathfrak{q}^{\perp}+2 r^{1 / 2} \mathfrak{h} * \mathfrak{q}^{\perp}\right.
$$

$$
\left.+\mathfrak{D}^{\prime} \mathfrak{q}+2 r^{1 / 2} \mathfrak{h}^{\prime} * \mathfrak{q}-\left(\mathfrak{v}^{\prime}-\mathfrak{v}_{\mathfrak{h}}^{\prime}\right)\right)=0
$$

where $\mathfrak{D}^{\prime}, \mathfrak{h}^{\prime}, \mathfrak{v}^{\prime}$ and $\mathfrak{v}_{\mathfrak{h}}^{\prime}$ denote the respective derivatives of $\mathfrak{D}, \mathfrak{h}, \mathfrak{v}$ and $\mathfrak{v}_{\mathfrak{h}}$ in that direction $\xi^{\prime}$. Given (6-3), Lemma 6.1, the second bullet in Lemma 6.3 and the bound on $\mathfrak{q}$ given by the second bullet in the proposition, it follows from (6-52) that

$$
\left\|\mathfrak{q}^{\prime}\right\|_{\mathbb{H}} \leq c_{0}\left(\left\|\mathfrak{D}^{\prime} \mathfrak{q}\right\|_{2}+r^{1 / 2}\left\|\mathfrak{h}^{\prime} * \mathfrak{q}\right\|_{2}+\left\|\mathfrak{v}^{\prime}-\mathfrak{v}_{\mathfrak{h}}^{\prime}\right\|_{2}\right),
$$

when $r \geq c_{0}$ and when $\|\xi\|_{\mathcal{K}} \leq c_{0}^{-1}$. A bound by $c_{0} r^{-1 / 2}$ on the right hand side of (6-53) is derived using much the same sorts of arguments that are used to obtain the second two bullets in Lemma 6.3. The details of these arguments are left to the reader.

What follows is a derivation of the bounds on $\|\mathfrak{q}\|_{*}$ and $\left\|\mathfrak{q}^{\prime}\right\|_{*}$ that are asserted by the last two bullets of Proposition 6.4. To start, fix $p \in \mathbb{R} \times M$ and $\rho>0$. Suppose that $\mathfrak{f}$ is, for the moment any given element of $\mathbb{H}$. Define

$$
\mathfrak{m}_{\mathfrak{f}}(p, \rho)=\int_{\operatorname{dist}(p, \cdot)<\rho}\left(\left|\nabla^{*} \mathfrak{f}\right|^{2}+\rho^{-2}|\mathfrak{f}|^{2}\right) .
$$

The last two bullets of Proposition 6.4 assert that $\mathfrak{m}_{\mathfrak{q}}(p, \rho)$ and $\mathfrak{m}_{\mathfrak{q}^{\prime}}(p, \rho)$ are respectively bounded by $c_{0} r^{-1}\left(r^{-1 / 2+8 \sigma}+\|\xi\|_{\mathcal{K}}\right)^{2} \rho^{v}$ and $c_{0} r^{-1} \rho^{v}$. The proof that this is so requires that $v$ is small; an upper bound is derived in the proof of the forthcoming Lemma 6.5.

To set the stage for Lemma 6.5, suppose for the moment that $\mathfrak{q} \in \mathbb{H}_{\xi}^{\perp}$ is not the element given by Proposition 6.4, but that it does obey the equation

$$
\mathfrak{D q}=w_{\mathfrak{q}}=\Pi_{\xi} \mathfrak{D q}-\left(1-\Pi_{\xi}\right)\left(r^{1 / 2} \mathfrak{f} * \mathfrak{q}+2 r^{1 / 2} \mathfrak{h} * \mathfrak{q}\right)+\mathfrak{u},
$$


where $\mathfrak{f} \in \mathbb{H}$ and where $\mathfrak{u} \in \mathbb{L}_{\xi} \frac{\perp}{\xi}$. Here, and in the rest of this proof, $\Pi_{\xi}$ is viewed as a map from $\mathbb{L}$ to itself, rather than as a map from $\mathbb{L}$ to $L^{2}$.

Lemma 6.5 The constant $\kappa$ from Lemmas 6.1-6.3 can be chosen so as to guarantee the following: Suppose that $v<\kappa^{-1}$, that $r \geq \kappa$, and that $\xi \in \mathcal{K}_{*}$ and $\|\xi\|_{\infty} \leq \kappa^{-1}$. Suppose in addition that the element $\mathfrak{f} \in \mathbb{H}$ has $\|\mathfrak{f}\|_{\mathbb{H}} \leq \kappa^{-1} r^{-1 / 2}$. Finally, suppose that $\mathfrak{u} \in \mathbb{L}_{\xi}^{\perp}$ and that there exist constants $\mathfrak{m}_{\mathbb{L}}$ and $\mathfrak{m}_{*}$ such that $\|\mathfrak{u}\|_{2}^{2} \leq \mathfrak{m}_{\mathbb{L}}$ and

$$
\int_{\operatorname{dist}(p, \cdot)<\rho}|\mathfrak{u}|^{2} \leq \mathfrak{m}_{*} \rho^{v}
$$

for each $p \in \mathbb{R} \times M$ and $\rho>0$. If $\mathfrak{q} \in \mathbb{H} \frac{\perp}{\xi}$ obeys (6-55), then $\|\mathfrak{q}\|_{\mathbb{H}}^{2} \leq \kappa \mathfrak{m}_{\mathbb{L}}$ and $\|\mathfrak{q}\|_{*}^{2} \leq \kappa\left(\mathfrak{m}_{*}+\left(r^{-1 / 4}+\|\xi\|_{\mathcal{K} *}^{2}\right) \mathfrak{m}_{\mathbb{L}}\right)$.

This lemma is proved momentarily.

The proofs of the asserted bounds on $\|\mathfrak{q}\|_{*}$ and $\left\|\mathfrak{q}^{\prime}\right\|_{*}$, and of Lemma 6.5 use the following three part digression. This digression supplies what are needed to bound the $L^{2}$ norm of the projection $\Pi_{\xi}(\cdot)$ or its directional derivative over any given ball in $\mathbb{R} \times M$.

Part 1 Fix an element $C \in \mathcal{C}$ that is not $\mathbb{R}$-invariant and consider what $\Pi_{\xi}$ does to a smooth section of $i T^{*} M \oplus \mathbb{S} \oplus i \mathbb{R}$ with compact support where $|s| \leq 2 R$ on $C$. To this end, write the restriction as $\mathfrak{f}=\left(\mathfrak{f}_{0}, \mathfrak{f}_{1}\right)$ with $\mathfrak{f}_{0,1}$ a section of $\mathbb{V}_{C 0,1}$. Then $\Pi_{\xi} \mathfrak{f}$ is equal to $t_{\xi}(\theta[\mathfrak{f}])$ where $\left.\theta[\mathfrak{f}]=\left(\left(\theta_{C}\right)_{C \in \mathcal{C}},\left(\theta_{\gamma-}\right)_{\gamma \in \Xi_{\Sigma_{-}}}\right),\left(\theta_{\gamma+}\right)_{\gamma \in \Xi_{\Sigma_{+}}}\right)$has only the nonzero component $\theta_{C}[f]$ with the latter defined at $p \in C-\left(\bigcup_{\mathcal{E} \in \mathcal{E}_{C}} \mathcal{E}_{2 R}\right)$ from an integral over the $|\mathfrak{s}| \leq \rho_{C}$ part of fiber of $N$ at $p$. This integral can be written as

$$
\left.\theta_{C}[\mathfrak{f}]\right|_{p}=\left(1+z_{p}\right) \int_{\left.N\right|_{p}} \chi_{C} \theta_{1 \xi, r}^{\dagger} \mathfrak{f}_{0},
$$

where $\theta_{1 \xi, r}=(1 / \sqrt{\pi})\left(\left(2^{-1 / 2} r^{1 / 2}\left(1-\left|\alpha^{C \xi, r}\right|^{2}\right), \partial_{A} V_{C \xi, r} \alpha^{C \xi, r}\right)\right.$ and where $\left|z_{p}\right| \leq$ $c_{0} r^{-1 / 2}$. The integration is defined using the metric that is pulled back from $\mathbb{R} \times M$ by the exponential map $e_{C}$. Analogous integrals define the components of $\theta[\mathfrak{f}]$ in the generic case.

Part 2 This part of the digression introduces notation that is used subsequently in this and the next subsection. To start, $D_{\rho}$ for $\rho>0$ denotes a disk of radius $\rho$ that lies either

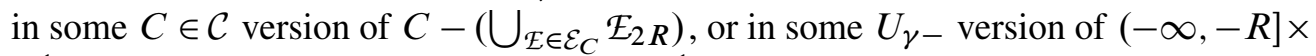
$S^{1}$, or in some $U_{\gamma+}$ version of $[R, \infty) \times S^{1}$. With $D_{\rho}$ specified, use $\pi$ to denote the projection from $e_{C^{-1}}\left(U_{C}\right)$ to $D_{\rho}$ when $D_{\rho} \subset C$, the projection to $(-\infty,-R] \times S^{1}$ when $D_{\rho}$ is in some $U_{\gamma-}$, or the projection to $[R, \infty) \times S^{1}$ when $D_{\rho}$ is in some $U_{\gamma+}$. 
As a final piece of notation, use $\theta_{r}$ to denote the function $\hat{\chi} r^{1 / 2} e^{-\sqrt{r} \operatorname{dist}(\Sigma, \cdot) / 100}$ where $\hat{\chi}$ here denotes $\chi_{C}$ or $\chi_{\gamma}$ or $\chi_{\gamma}+$ depending on whether $D_{\rho}$ is in a component $C$ of $\Sigma$,

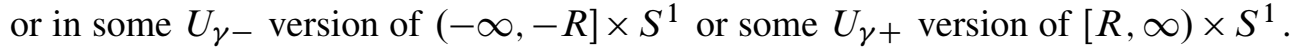

Part 3 Let $D_{\rho}$ be as above and let $D_{2 \rho}$ denote the concentric disk of radius $2 \rho$. If $C \in \mathcal{C}$ is not $\mathbb{R}$-invariant and $D_{\rho} \subset C$, let $\hat{\theta}$ denote a section of $\mathbb{V}_{0 C} \oplus \mathbb{V}_{1 C}$ over $\pi^{-1}\left(D_{2 \rho}\right)$. If $C \in \mathcal{C}$ is $\mathbb{R}$-invariant and $D_{\rho} \subset C$, let $\hat{\theta}$ denote a map from $D_{2 \rho} \times \mathbb{C}$ to $\mathbb{V}_{0} \oplus \mathbb{V}_{1}$. If $D_{\rho}$ is in any $U_{\gamma \pm}$ version of $\mathbb{R} \times S^{1}$, let $\hat{\theta}$ again denote a map from $D_{2 \rho} \times C$ to $\mathbb{V}_{0} \oplus \mathbb{V}_{1}$. In all cases, assume that $|\hat{\theta}| \leq f \theta_{r}$ where $f$ is an $L^{2}$ function with compact support on $D_{2 \rho}$.

Granted that such is the case, then $\hat{\theta}$ can be viewed as an element in $\mathbb{L}$.

Now suppose that $\mathfrak{f} \in \mathbb{L}$. Fix $p \in \mathbb{R} \times M$ and $\rho>0$. Define $\Pi^{\theta} \mathfrak{f} \in \mathbb{L}$ by

$$
\Pi^{\theta} \mathfrak{f}=\hat{\theta} \int_{\pi^{1}(\cdot)} \hat{\theta}^{\dagger} \mathfrak{f}
$$

The integral of $\Pi^{\theta} \mathfrak{f}$ over the radius $\rho$ ball centered at $p$ is zero unless this ball intersects the support of $\hat{\theta}$ in which case

$$
\int_{\operatorname{dist}(p, \cdot)<\rho}\left|\Pi^{\theta} \mathfrak{f}\right|^{2} \leq c_{0} \min \left(1, r \rho^{2}\right) \int_{z \in D_{2 p}}\left(f^{2}\left(\int_{\pi^{-1}(z)} \theta_{r}|\mathfrak{f}|\right)^{2}\right),
$$

where $D_{\rho}$ is a disk as described above.

Consider as an example $\Pi_{\xi} \mathfrak{f}$. The square of the $L^{2}$ norm of $\Pi_{\xi} \mathfrak{f}$ over a ball of radius $\rho$ in $\mathbb{R} \times M$ is zero unless said ball intersects some $C \in \mathcal{C}$ version of $U_{C}$ or some $\gamma \in \Xi_{\gamma-}$ version of $U_{\gamma-}$ or some $\gamma \in \Xi_{\gamma+}$ version of $U_{\gamma+}$. If so, then

$$
\int_{\operatorname{dist}(p, \cdot)<\rho}\left|\Pi_{\xi} \mathfrak{f}\right|^{2} \leq c_{0} \min \left(1, r \rho^{2}\right) \int_{z \in D_{2 p}}\left(\int_{\pi^{-1}(z)} \theta_{r}|\mathfrak{f}|\right)^{2} .
$$

To use Lemma 6.5 for the proof of the fifth bullet of Proposition 6.4, note that $\mathfrak{q}$ from Proposition 6.4 obeys (6-55) with $\mathfrak{f}=\mathfrak{q}$ and with $\left.\mathfrak{u}=r^{-1 / 2}\left(1-\Pi_{\xi}\right)\left(\mathfrak{v}-\mathfrak{v}_{\mathfrak{h}}\right)\right)$. Given what is said in the digression, and given the definition in (2-27) of $\|\cdot\|_{\mathcal{K} *}$, arguments much like those that were used to prove the third bullet of Lemma 6.3 bound the integral in (6-56) by $c_{0} r^{-1 / 2}\left(r^{-1 / 2+8 \sigma}+\|\xi\|_{\mathcal{K}}\right) \rho^{v}$. The details involve nothing new and are left to the reader.

Lemma 6.5 can also be used to derive the bound for the $\|\cdot\|_{*}$ norm of the directional derivative of $\mathfrak{q}$ that is asserted in the sixth bullet of Proposition 6.4. To this end, fix $\xi$ 
and let $\xi^{\prime} \in \mathcal{K}$ denote a vector with unit norm. Let $\mathfrak{q}^{\prime}$ again denote the derivative of $\mathfrak{q}$ in the direction of $\xi^{\prime}$; and again write $\mathfrak{q}^{\prime}=\mathfrak{q}^{\perp}-\Pi_{\xi}^{\prime} \mathfrak{q}$.

The square of the $L^{2}$ norm of $\Pi_{\xi}^{\prime} \mathfrak{q}$ over the ball of radius $\rho$ centered at $p$ is bounded by $c_{0}$ times the $\mathfrak{f}=\mathfrak{q}$ version of the right hand side of (6-58). As a consequence, $\rho^{-2}$ times its $L^{2}$ norm is bounded by $c_{0}\|\mathfrak{q}\|_{*}^{2} \rho^{v}$. Meanwhile, the square of the $L^{2}$ norm of $\nabla^{*}\left(\Pi_{\xi}^{\prime} \mathfrak{q}\right)$ over such a ball can be written as the sum of two terms. The first involves $\Pi_{\xi}^{\prime}$ acting on derivatives of $\mathfrak{q}$, and the second involves derivatives of $\Pi_{\xi}^{\prime}$ acting on $\mathfrak{q}$. The square of the $L^{2}$ norm of the former is bounded by $c_{0}$ times the $\mathfrak{f}=\nabla^{*} \mathfrak{q}$ version of the right hand side of (6-58); thus it is also bounded by $c_{0}\|\mathfrak{q}\|_{*}^{2} \rho^{v}$. Given (2-11) and (2-12), it follows from (6-59) that the square of the $L^{2}$ norm of the latter term is bounded by

$$
c_{0} \min \left(1, r \rho^{2}\right) \int_{z \in D_{2 \rho}}\left(\left(|\nabla \xi|^{2}+\left|\nabla \xi^{\prime}\right|^{2}+1\right)\left(\int_{\pi^{-1}(z)} \theta_{r}|\mathfrak{q}|\right)^{2}\right) .
$$

It follows using [3, Lemmas 2.9 and 5.4.1] that what is written in (6-61) is also bounded by $c_{0}\|\mathfrak{q}\|_{*}^{2} \rho^{v}$.

To say something about the $\|\cdot\|_{*}$ norm of $\mathfrak{q}^{\perp}$, note first that the latter obeys (6-52) and that (6-52) is the version of (6-55) with $\mathfrak{u}=\left(1-\Pi_{\xi}\right)\left(\mathfrak{D}^{\prime} \mathfrak{q}+2 r^{1 / 2} \mathfrak{h}^{\prime} * \mathfrak{q}-\left(\mathfrak{v}^{\prime}-\mathfrak{v}_{\mathfrak{h}}^{\prime}\right)\right)$. The constructions that are used to prove the last two bullets of Lemma 6.3 can be differentiated, so to speak, and thus used to establish that this version of $\mathfrak{u}$ obeys (6-56) with $\mathfrak{m}_{*} \leq c_{0} r^{-1 / 2}$. The details are straightforward and left to the reader.

\section{6.f Proof of Lemma 6.5}

It follows from Lemma 6.1, the second bullet of Lemma 6.3 and (6-3) that $\|\mathfrak{q}\|_{\mathbb{H}}^{2} \leq$ $c_{0}\left(r^{1 / 2}\|\mathfrak{f}\|_{\mathbb{H}}\|\mathfrak{q}\|_{\mathbb{H}}+\mathfrak{m}_{\mathbb{L}}\right)$. As a consequence, $\|\mathfrak{q}\|_{\mathbb{H}}^{2} \leq c_{0} \mathfrak{m}_{\mathbb{L}}$ if $\|\mathfrak{f}\|_{\mathbb{H}}^{2} \leq c_{0}^{-1} r^{-1}$. To bound $\|\mathfrak{q}\|_{*}^{2}$ digress momentarily so as to define for each $\varepsilon>0$,

$$
\|\mathfrak{q}\|_{* \varepsilon}^{2}=\sup _{p \in \mathbb{R} \times M} \sup _{\rho \in(\varepsilon, 1)} \rho^{-v} \int_{\operatorname{dist}(p, \cdot)<\rho}\left(\left|\nabla^{*} \mathfrak{q}\right|^{2}+\rho^{-2}|\mathfrak{q}|^{2}\right) .
$$

Note that $\|\mathfrak{q}\|_{*}=\lim _{\varepsilon \rightarrow 0}\|\mathfrak{q}\|_{* \varepsilon}$. Since $\|\mathfrak{q}\|_{* \varepsilon}<\varepsilon^{-v / 2}\|\mathfrak{q}\|_{\mathbb{H}}$, what is at issue is the existence of a suitable $r$ and $\varepsilon$ independent bound for each $\|\mathfrak{q}\|_{* \varepsilon}$. The derivation of such a bound has eight steps.

Step 1 It follows using a Sobolev inequality that

$$
r \int_{\operatorname{dist}(p, \cdot)<\rho}|\mathfrak{f} * \mathfrak{q}|^{2} \leq c_{0} r\|\mathfrak{f}\|_{\mathbb{H}}^{2}\|\mathfrak{q}\|_{* \varepsilon}^{2} \rho^{v} .
$$


Here is the Sobolev inequality: Let $\mathfrak{q}$ denote any given element in $H$. Then

$$
\left(\int_{\operatorname{dist}(p, \cdot)<\rho}|\mathfrak{q}|^{4}\right)^{1 / 2} \leq c_{0} \int_{\operatorname{dist}(p, \cdot)<\rho}\left(\left|\nabla^{*} \mathfrak{q}\right|^{2}+\rho^{-2}|\mathfrak{q}|^{2}\right),
$$

where $c_{0}$ is independent of $p, \rho$ and the connection that defines $\nabla^{*}$.

Meanwhile,

(6-65) $r \int_{\operatorname{dist}(p, \cdot)<\rho}|\mathfrak{h} * \mathfrak{q}|^{2} \leq c_{0} R\left(\int_{\operatorname{dist}(p, \cdot)<\rho}|\mathfrak{h}|^{4}\right)^{1 / 2}\|\mathfrak{q}\|_{* \varepsilon}^{2} \rho^{v} \leq c_{0} r^{-\sigma / 4}\|\mathfrak{q}\|_{* \varepsilon}^{2} \rho^{v}$.

Indeed, this follows by first using Hölder's inequality, and then using the properties of the various contributions to $\mathfrak{h}$ that are described in the proof of Lemma 6.3 to bound the square of the $L^{4}$ norm of $\mathfrak{h}$ over any radius $\rho$ ball by $c_{0} r^{-1-\sigma / 4}$.

Step 2 This step considers

$$
\int_{\operatorname{dist}(p, \cdot)<\rho}\left|\Pi_{\xi} \mathfrak{D q}\right|^{2} .
$$

To begin the analysis, write $\Pi_{\xi} \mathfrak{D}=\mathfrak{D} \Pi_{\xi}+\left[\Pi_{\xi}, \mathfrak{D}\right]$. This done, it follows from (6-59) that this integral can be bounded by $c_{0} \min \left(1, r \rho^{2}\right)\left(\mathcal{A}_{1}+\mathcal{A}_{2}\right)$ with

$$
\begin{aligned}
& \mathcal{A}_{1}=\int_{z \in D_{2 \rho}}\left(\left(|\nabla \xi|^{2}+|\xi|^{2}\right)\left(\int_{\pi^{-1}(z)} \theta_{r}|\mathfrak{q}|\right)^{2}\right) . \\
& \mathcal{A}_{2}=\rho_{*}^{2} \int_{z \in D_{2 \rho}}\left(\int_{\pi^{-1}(z)} \theta_{r}(|\nabla \mathfrak{q}|+|\mathfrak{q}|)^{2}\right) .
\end{aligned}
$$

Indeed, as $\Pi_{\xi} \mathfrak{q}=0$, the integrand in (6-66) is $\left|\left[\Pi_{\xi}, \mathfrak{D}\right] \mathfrak{q}\right|^{2}$. The commutator has zeroth order parts which arise when derivatives in $\mathfrak{D}$ act on $\xi$. The latter are accounted by $\mathcal{A}_{1}$. The other parts to the commutator arise from the terms in (6-6) and (6-7) that are denoted by $\mathfrak{r}_{00}, \mathfrak{r}_{01}, \mathfrak{r}_{10}$ and $\mathfrak{r}_{11}$. These are accounted for by $\mathcal{A}_{2}$.

It follows from [3, Lemmas 2.9 and 5.4.1] that $\mathcal{A}_{1}$ is bounded by

$$
c_{0} \rho^{v}\|\xi\|_{\mathcal{K}}^{2}\|\mathfrak{q}\|_{\mathbb{H}}^{2} \leq c_{0}\|\xi\|_{\mathcal{K}^{2}}^{2} \mathfrak{m}_{\mathbb{L}} \rho^{v}
$$

Meanwhile, $\mathcal{A}_{2}$ is bounded by $c_{0} \rho_{*}^{2}\|\mathfrak{q}\|_{\mathbb{H}}^{2} \leq c_{0} r^{-3 / 4} \mathfrak{m}_{\mathbb{L}}$. Thus, in the case when $\rho \geq r^{-1 / 2-v}$,

$$
\mathcal{A}_{2} \leq \mathcal{A}_{2}\left(\rho r^{1 / 2+v}\right)^{v} \leq c_{0} \mathfrak{m}_{\mathbb{L}} r^{-1 / 2} \rho^{v} .
$$

When $\rho \leq r^{-1 / 2-v}$, then $\min \left(1, r \rho^{2}\right) \leq \rho^{v}$ and so $\min \left(1, r \rho^{2}\right) \mathcal{A}_{2}$ is again bounded by what is written on the right hand side of (6-69). Thus if $v \ll \sigma / 4$, then the integral in (6-66) is bounded by $c_{0}\left(r^{-1 / 2}+\|\xi\|_{\mathcal{K}}^{2}\right) \mathfrak{m}_{\mathbb{L}} \rho^{v}$. 
Step 3 This step considers $r \int_{\operatorname{dist}(p, \cdot)<\rho}\left|\Pi_{\xi}(\mathfrak{h} * \mathfrak{q})\right|^{2}$. According to (6-60), this integral is bounded by $c_{0} \min \left(1, r \rho^{2}\right) \mathcal{A}_{3}$ where

$$
\mathcal{A}_{3}=r \int_{z \in D_{2 p}}\left(\int_{\pi^{-1}(z)} \theta_{r}|\mathfrak{h} * \mathfrak{q}|\right)^{2} .
$$

The contribution to $\mathcal{A}_{3}$ from $\mathfrak{b}_{ \pm, 0}$ is bounded by

(6-71) $c_{0} r \int_{\left|s-s_{p}\right|<4 \rho} \int_{M}\left|\mathfrak{b}_{ \pm, 0}\right|^{2}|\mathfrak{q}|^{2} \leq c_{0} r \int_{\left|s-s_{p}\right|<4 \rho}\left(\int_{M}\left|\mathfrak{b}_{ \pm, 0}\right|^{4}\right)^{1 / 2}\left(\int_{M}|\mathfrak{q}|^{4}\right)^{1 / 2}$.

Here, $s_{p}$ denotes the value of $s$ at the point $p$. As the $L^{4}$ norm of $\mathfrak{b}_{ \pm, 0}$ over $S^{1} \times M$ is bounded by $r^{-5 / 8}$, the right hand side of (6-71) is bounded by

$$
c_{0} r^{-1 / 4} \int_{\left|s-s_{p}\right|<4 \rho}\left(\int_{M}|\mathfrak{q}|^{4}\right)^{1 / 2} \leq c_{0} r^{-1 / 4} \rho^{1 / 2}\|\mathfrak{q}\|_{\mathbb{H}}^{2} .
$$

The expression on the right side of (6-72) is bounded by $c_{0} \mathfrak{m}_{\mathbb{L}} r^{-1 / 4} \rho^{v}$. Meanwhile, the remaining contributions to $\mathcal{A}_{3}$ are bounded using (6-35) and (6-42) by

$$
\int_{z \in D_{2 \rho}} \int_{\pi^{-1}(z)} r^{-1 / 2} \theta_{r}|\mathfrak{q}|^{2} \leq \rho r^{-1 / 2}\|\mathfrak{q}\|_{\mathbb{H}}^{2}
$$

This is also bounded by $c_{0} \mathfrak{m}_{\mathbb{L}} r^{-1 / 4} \rho^{v}$.

Step 4 This step discusses the term $r \int_{\operatorname{dist}(p, \cdot)<\rho}\left|\Pi_{\xi}(\mathfrak{f} * \mathfrak{q})\right|^{2}$. According to (6-60), the latter is bounded by $c_{0} \min \left(1, r \rho^{2}\right) \mathcal{A}_{4}$ where

$$
\mathcal{A}_{4}=r \int_{z \in D_{2 \rho}}\left(\int_{\pi^{-1}(z)} \theta_{r}|\mathfrak{f}|^{2} \int_{\pi^{-1}(z)} \theta_{r}|\mathfrak{q}|^{2}\right) .
$$

There are two cases to consider. In the first, $\rho \geq r^{-1 / 2}$. In this case, the arguments from Step 1 can be repeated to find that $\mathcal{A}_{4}$ is bounded by the right hand side of (6-63). The second case is that where $\rho<r^{-1 / 2}$. To deal with this case, fix an identification of $\pi^{-1}\left(D_{2 \rho}\right)$ with $D_{2 \rho} \times \mathbb{C}$. Let $\Lambda \subset \mathbb{C}$ denote the square lattice $\frac{1}{2} \rho \mathbb{Z}^{2}$. It follows now that

$$
\begin{aligned}
\mathcal{A}_{4} & \leq r c_{0} \sum_{u \in \Lambda}\left(e^{-\sqrt{r}|u| / c_{0}} \int_{D_{2 \rho} \times D_{2 \rho}(u)}|\mathfrak{f}|^{2}|\mathfrak{q}|^{2}\right) \\
& \leq r c_{0}\left(\sum_{u \in \Lambda} e^{-\sqrt{r}|u| / c_{0}}\right)\|\mathfrak{f}\|_{\mathbb{H}}^{2}\|\mathfrak{q}\|_{* \varepsilon}^{2} \rho^{v} .
\end{aligned}
$$

Here, $D_{2 \rho}(u) \subset \mathbb{C}$ denotes the disk of radius $2 \rho$ with center at the point $u$. The key observation here is that sum that appears on the far right in (6-75) is bounded by $c_{0}\left(r \rho^{2}\right)^{-1}$. Thus, $\min \left(1, r \rho^{2}\right) \mathcal{A}_{4}$ is bounded by the right hand side of (6-63) in the case $\rho<r^{-1 / 2}$. 
Step 5 Set $z=\left(r^{-\sigma / 4}+r^{1 / 2}\|\mathfrak{f}\|_{\mathbb{H}}\right)^{2}$ and $\mathfrak{m}_{\xi}=\mathfrak{m}_{*}+\left(r^{-1 / 4}+\|\xi\|_{\mathcal{K}}^{2}\right) \mathfrak{m}_{\mathbb{L}}$. With the equation for $\mathfrak{q}$ written as $\mathfrak{D} \mathfrak{q}=\mathfrak{w}_{\mathfrak{q}}$, it has now been established that

$$
\rho^{-v} \int_{\operatorname{dist}(p, \cdot)<\rho}\left|\mathfrak{w}_{\mathfrak{q}}\right|^{2} \leq c_{0}\left(z\|\mathfrak{q}\|_{* \varepsilon}^{2}+\mathfrak{m}_{\xi}\right)
$$

for each $\rho>\varepsilon$ and point $p \in \mathbb{R} \times M$.

To proceed from here, fix $T>1$ and consider first the case where $\rho<T^{-1} r^{-1 / 2}$. Let $\mathcal{X}$ denote the function on $\mathbb{R} \times M$ with compact support in the ball of radius $2 \rho$ centered at $p$ that equals 1 on the concentric ball of radius $\rho$ and otherwise equals $\left(2-\rho^{-1} \operatorname{dist}(p, \cdot)\right)$. The section $\mathfrak{q}_{\rho}=\chi \mathfrak{q}$ of $i T^{*} M \oplus \mathbb{S} \oplus i \mathbb{R}$ obeys

$$
\mathfrak{D} \mathfrak{q}_{\rho}=s \mathfrak{q}+\chi \mathfrak{w}_{\mathfrak{q}},
$$

where $s$ is supported where $\rho \leq \operatorname{dist}(p, \cdot) \leq 2 \rho$ and obeys $|s| \leq c_{0} \rho^{-1}$. It follows as a consequence of what has been derived in Steps 1-3 that

$$
\int\left|\mathfrak{D} \mathfrak{q}_{\rho}\right|^{2} \leq c_{0} \int_{\rho<\operatorname{dist}(p, \cdot)<2 \rho} \operatorname{dist}(p, \cdot)^{-2}|\mathfrak{q}|^{2}+c_{0}\left(z\|\mathfrak{q}\|_{* \varepsilon}^{2}+\mathfrak{m}_{\xi}\right) \rho^{v}
$$

Use $\mathfrak{D}_{*}$ to denote the version of the operator $\mathfrak{D}$ that is defined using $\xi=0$. Write $\mathfrak{D}=\mathfrak{D}_{*}+\sqrt{r} \mathfrak{y}_{\xi}$ and then use the Bochner-Weitzenboch formula for $\mathfrak{D}_{*}$ to prove that

$$
\int\left|\mathfrak{D} \mathfrak{q}_{\rho}\right|^{2} \geq c_{0}^{-1} \int\left|\nabla^{*} \mathfrak{q}_{\rho}\right|^{2}-c_{0} \int\left(1+r e^{-\sqrt{r} \operatorname{dist}(\Sigma, \cdot) / 100}\right)\left|\mathfrak{q}_{\rho}\right|^{2} .
$$

As the smallest Dirichlet eigenvalue of $\nabla^{* \dagger} \nabla^{*}$ on the radius $\rho$ ball is no less than $c_{0}^{-1} \rho^{-2}$, this last inequality implies that

$$
\int\left|\mathfrak{D} \mathfrak{q}_{\rho}\right|^{2} \geq c_{0}^{-1} \int\left|\nabla^{*} \mathfrak{q}_{\rho}\right|^{2}
$$

when $T \geq c_{0}$. What is written in (6-78) and (6-80) imply the following: If $r \geq c_{0}$, $T>c_{0}$ and $\|\xi\|_{\mathcal{K}} \leq c_{0}^{-1}$, then

$$
\int\left|\nabla^{*} \mathfrak{q}_{\rho}\right|^{2} \leq c_{0} \int_{\rho<\operatorname{dist}(p, \cdot)<2 \rho} \operatorname{dist}(p, \cdot)^{-2}|\mathfrak{q}|^{2}+c_{0}\left(z\|\mathfrak{q}\|_{* \varepsilon}^{2}+\mathfrak{m}_{\xi}\right) \rho^{v} .
$$

Meanwhile, a standard Sobolev inequality (proved by integrating by parts in spherical coordinates) finds

$$
\int\left|\nabla^{*} \mathfrak{q}_{\rho}\right|^{2} \geq \int_{\operatorname{dist}(p, \cdot)<\rho} \operatorname{dist}(p, \cdot)^{-2}|\mathfrak{q}|^{2} .
$$

As a consequence, (6-81) and (6-82) imply that the function $\mathfrak{m}_{\mathfrak{q}}(p, \rho)$ obeys

$$
\mathfrak{m}_{\mathfrak{q}}(p, \rho) \leq \lambda \mathfrak{m}_{\mathfrak{q}}(p, 2 \rho)+c_{0}\left(z\|\mathfrak{q}\|_{* \varepsilon}^{2}+\mathfrak{m}_{\xi}\right) \rho^{v} \quad \text { for } \quad \varepsilon<\rho<K^{-1} r^{-1 / 2},
$$

where $\lambda<1$ is independent of $\mathfrak{f}, \mathfrak{u}, v, \rho, p$ and $r$. 
The fact that $\lambda$ is independent of $v$ has the following consequence: If $v<c_{0}^{-1}$, then $2^{v} \lambda<1 /\left(1+c_{0}^{-1}\right)$. Granted that $v$ obeys this last constraint, iterate (6-83) some $N$ times where $N$ is such that $2^{N} \rho \in\left[\frac{1}{2} T^{-1} r^{-1 / 2}, T^{-1} r^{-1 / 2}\right]$. This iteration process finds that $\mathfrak{m}_{\mathfrak{q}}(p, \rho)$ is bounded by $c_{0}\left(z\|\mathfrak{q}\|_{* \varepsilon}^{2}+\mathfrak{m}_{\xi}\right) \rho^{v}+c_{0} \lambda^{N} \mathfrak{m}_{\mathfrak{q}}\left(p, T^{-1} r^{-1 / 2}\right)$. This is to say that

$$
\mathfrak{m}_{\mathfrak{q}}(p, \rho) \leq c_{0}\left(p r^{1 / 2}\right)^{\delta} \mathfrak{m}_{\mathfrak{q}}\left(p, 2 T r^{-1 / 2}\right)+c_{0}\left(z\|\mathfrak{q}\|_{* \varepsilon}^{2}+\mathfrak{m}_{\xi}\right) \rho^{v},
$$

where the exponent $\delta$ that appears in the right most term is positive and independent of $\mathfrak{f}, \mathfrak{u}, v, \rho, p$ and $r$ if $v \leq 1 / c_{0}$. Note the replacement of $T^{-1}$ by $2 T$ in the right most term. This is done for the following reason: Since there are on the order of $T^{8}$ balls of radius $T^{-1} r^{-1 / 2}$ inside the ball of radius $T r^{-1 / 2}$, any $\rho \leq T r^{-1 / 2}$ version of $\mathfrak{m}_{\mathfrak{q}}(p, \rho)$ is bounded by $c_{0} T^{8}$ times what is written on the right hand side of (6-84).

The discussion returns to (6-84) in Step 7.

Step 6 Suppose now that $\rho>T r^{-1 / 2}$. Introduce $\chi$ as before and write $\mathfrak{q}_{\rho}=\chi \mathfrak{q}$. Write $\mathfrak{q}_{\rho}=\left(1-\Pi_{\xi}\right) \mathfrak{q}_{\rho}+\Pi_{\xi} \mathfrak{q}_{\rho}$. Use first order Taylor's approximations for $\chi$ near $\Sigma$ to see that

$$
\left\|\Pi_{\xi} \mathfrak{q}_{\rho}\right\|_{2}^{2}<c_{0}(r \rho)^{-2} \int_{\rho \leq \operatorname{dist}(p, \cdot) \leq 2 \rho}|\mathfrak{q}|^{2}+c_{0} e^{-\sqrt{r} \rho / c_{0}}\left\|\mathfrak{q}_{\rho}\right\|_{2}^{2} .
$$

This uses (2-1) and (2-2) plus (2-4) and (2-5) when $p \in U_{\gamma \pm}$. By the same token, $\left\|\nabla^{*}\left(\Pi_{\xi} \mathfrak{q}_{\rho}\right)\right\|_{2}^{2}$ is bounded by

$$
\begin{aligned}
c_{0}\left(p^{-2} \int_{\rho \leq \operatorname{dist}(p, \cdot) \leq 2 \rho}|\mathfrak{q}|^{2}+\left(r^{1 / 2} \rho\right)^{-2}\right. & \left.\int_{\rho \leq \operatorname{dist}(p, \cdot) \leq 2 \rho}\left|\nabla^{*} \mathfrak{q}\right|^{2}\right) \\
& +c_{0} e^{-\sqrt{r} \rho / c_{0}}\left(r\left\|\mathfrak{q}_{\rho}\right\|_{2}^{2}+\left\|\nabla^{*} \mathfrak{q}_{\rho}\right\|_{2}^{2}\right) .
\end{aligned}
$$

Meanwhile, Lemma 6.1 applied to $\left(1-\Pi_{\xi}\right) \mathfrak{q}_{\rho}$ finds

$$
\left\|\mathfrak{D}\left(\left(1-\Pi_{\xi}\right) \mathfrak{q}_{\rho}\right)\right\|_{2}^{2} \geq c_{0}^{-1}\left(\left\|\nabla^{*}\left(\left(1-\Pi_{\xi}\right) \mathfrak{q}_{\rho}\right)\right\|_{\mathbb{H}}^{2}+r\left\|\left(1-\Pi_{\xi}\right) \mathfrak{q}_{\rho}\right\|_{2}^{2}\right) .
$$

The preceding two inequalities imply that

$$
\begin{array}{r}
\left\|\mathfrak{D} \mathfrak{q}_{\rho}\right\|_{2}^{2}+\rho^{-2} \int_{\rho \leq \operatorname{dist}(p, \cdot) \leq 2 \rho}|\mathfrak{q}|^{2}+T^{-2} \int_{\rho \leq \operatorname{dist}(p, \cdot) \leq 2 \rho}\left|\nabla^{*} \mathfrak{q}\right|^{2} \\
\geq c_{0}^{-1}\left(\left\|\nabla^{*} \mathfrak{q}_{\rho}\right\|_{2}^{2}+r\left\|\mathfrak{q}_{\rho}\right\|_{2}^{2}\right) .
\end{array}
$$

Equations (6-88) with (6-76) and (6-77) imply that (6-83) holds with $\lambda<1$ a constant that is independent of $\mathfrak{f}, \mathfrak{u}, v, \rho, p$ and $r$ if $v<1 / c_{0}$ and $\rho<1 / c_{0}$. 
As before, the inequality in (6-83) can be iterated if $v<1 / c_{0}$. Iterated $N$ times with $N$ chosen so that $2^{N} \rho$ is $O(1)$; which is to say greater than $1 / c_{0}$. Doing so finds that

$$
\mathfrak{m}_{\mathfrak{q}}(p, \rho) \leq c_{0}\left(z\|\mathfrak{q}\|_{* \varepsilon}^{2}+\mathfrak{m}_{\xi}\right) \rho^{v}+\lambda^{N} \mathfrak{m}_{\mathfrak{q}}\left(p, 1 / c_{0}\right) .
$$

As $\mathfrak{m}_{\mathfrak{q}}\left(p, 1 / c_{0}\right) \leq c_{0}\|\mathfrak{q}\|_{\mathbb{H}}^{2} \leq c_{0} \mathfrak{m}_{\mathbb{L}}$, what is written in (6-89) implies that

$$
\mathfrak{m}_{\mathfrak{q}}(p, \rho) \leq c_{0}\left(z\|\mathfrak{q}\|_{* \varepsilon}^{2}+\mathfrak{m}_{\xi}\right)\left(\rho^{v}+\rho^{\delta^{\prime}}\right),
$$

where $\delta^{\prime}>0$ is independent of $v, \rho, p$ and $r$.

Step 7 Given that $v<1 / c_{0}$, what is written in (6-90) asserts that $\mathfrak{m}_{\mathfrak{q}}(p, \rho)<$ $c_{0}\left(z\|\mathfrak{q}\|_{* \varepsilon}^{2}+\mathfrak{m}_{\xi}\right) \rho^{v}$ for $\rho \geq T r^{-1 / 2}$. This understood, return to (6-84) to see that

$$
\mathfrak{m}_{\mathfrak{q}}(p, \rho) \leq c_{0}\left(\rho r^{1 / 2}\right)^{\delta} \mathfrak{m}_{*} r^{-v / 2}+\left(z\|\mathfrak{q}\|_{* \varepsilon}^{2}+\mathfrak{m}_{\xi}\right) \rho^{v}
$$

for $\rho$ such that $\varepsilon<\rho<T r^{-1 / 2}$. Here it is important to note that $\delta$ is independent of $v$ (as well as $\mathfrak{f}, \mathfrak{u}, \rho, p$ and $r$ ). Thus, if $v<\delta$, the (6-91) has $\mathfrak{m}_{\mathfrak{q}}(p, \rho) \leq$ $c_{0}\left(z\|\mathfrak{q}\|_{* \varepsilon}^{2}+\mathfrak{m}_{\xi}\right) \rho^{v}$ for all $\varepsilon<p \leq 1 / c_{0}$.

Step 8 The preceding implies that $\|\mathfrak{q}\|_{* \varepsilon}^{2} \leq c_{0} \mathfrak{m}_{\xi} /\left(1-c_{0} z\right)$. It follows as a consequence that $\|\mathfrak{q}\|_{* \varepsilon}^{2} \leq c_{0} \mathfrak{m}_{\xi}$ when $r \geq c_{0}$ and $r^{1 / 2}\|\mathfrak{f}\|_{\mathbb{H}}$ is bounded by $1 / c_{0}$. If such is the case, then $\|\mathfrak{q}\|_{*}^{2}=\lim _{\varepsilon>0}\|\mathfrak{q}\|_{* \varepsilon}^{2} \leq c_{0}\left(\mathfrak{m}_{*}+\left(r^{-1 / 4}+\|\xi\|_{\mathcal{K}}^{2}\right) \mathfrak{m}_{\mathbb{L}}\right)$ also.

\section{The construction of instantons: Part III}

The assumptions of Proposition 6.4 are taken as given in what follows; and thus its conclusions hold. View Proposition 6.4's vector $\mathfrak{b}$ as a function on $\mathcal{B}$. The task for this subsection is to find a point $\xi \in \mathcal{B}$ where

$$
\Pi_{\xi}\left(\mathfrak{D q}+r^{1 / 2} \mathfrak{q} * \mathfrak{q}+2 r^{1 / 2} \mathfrak{h} * \mathfrak{q}-\left(\mathfrak{v}-\mathfrak{v}_{\mathfrak{h}}\right)\right)=0 .
$$

Here, $\Pi_{\xi}$ is viewed as a map from $\mathbb{L}$ to $L^{2}$.

The next proposition says what is needed about solutions to (7-1).

Proposition 7.1 Fix $\Sigma$ as in Section 4.b satisfying all constraints. There exists $\kappa \geq 100$ and a finite dimensional, normed vector space $V_{0}$ which have the following properties: Make the constructions that lead to (7-1) with $R \geq \kappa$, with $r \geq \kappa$, and with the radius of Proposition 6.4's ball $\mathcal{B}$ less than $\kappa^{-1}$. There is a linear map q: $\mathcal{K} \rightarrow V_{0}$ such that

- $|\mathfrak{q}(\xi)| \leq \kappa\|\xi\|_{L^{2}}$.

- If $\lambda \in V_{0}$, then there is an element $\xi \in \mathfrak{q}^{-1}(\lambda)$ with $\|\xi\|_{\mathcal{K}} \leq \kappa|\lambda|$. 
- If $\lambda \in \mathfrak{q}(\mathcal{B})$, then there is a unique element in $\mathcal{B} \cap \mathfrak{q}^{-1}(\lambda)$ where (7-1) holds.

- This element, $\xi_{\lambda}$, obeys $\left\|\xi_{\lambda}\right\|_{\mathcal{K}} \leq \kappa\left(r^{-1 / 2+16 \sigma}+|\lambda|\right)$.

- The assignment $\lambda \rightarrow \xi_{\lambda}$ defines a smooth map from $\mathfrak{q}(\mathcal{B})$ to $\mathcal{B}$.

The vector space $V_{0}$ is determined by $\Sigma$. It is described in Section 7.f.

Sections 7.a-7.i supply the proof of Proposition 7.1. Together, Propositions 6.4 and 7.1 supply a pair $(\xi, \mathfrak{b})$ that have Properties 1 and 2 as described in Section 5.d. As is argued in Section 7.j, the $(\xi, \mathfrak{b})$ version of $(A, \psi)$ given in (5-19) is gauge equivalent to an instanton solution of (1-14) with the following large $|s|$ asymptotics: The $s \rightarrow-\infty$ limit is the solution of (1-13) that is constructed in Section 3 from the data $\Theta=\Theta_{-}$. Meanwhile, the $s \rightarrow \infty$ limit is gauge equivalent to the solution of (1-13) that is constructed in Section 3 using the data $\Theta=\Theta_{+}$. Section 7.k gives the proof of Theorem 1.2.

\section{7.a Proof of Proposition 7.1}

The proof this proposition uses perturbative techniques. What follows gives the details. To start, view $\Pi_{\xi}$ in (7-1) as a map from $\mathbb{L}$ to the Banach space $\mathcal{L}^{2}$. This understood, it follows from the fourth point of Proposition 6.4 that the left hand side of (7-1) defines a smooth map from $\mathcal{B}$ to $\mathcal{L}^{2}$. As it turns out, the norm that defines $\mathcal{L}^{2}$ is not strong enough for the purposes at hand. Here is a suitable replacement: Define $\mathcal{L}$ to be the Banach space that is obtained by completing the set of smooth elements in $\mathcal{L}^{2}$ using the norm that is defined by analog of (5-12) that has each $\|\cdot\|_{\mathcal{K}}$ norm replaced by the corresponding version of (2-29). Denote this norm by $\|\cdot\|_{\mathcal{L}}$.

As explained momentarily, the left hand side of (7-1) defines a smooth map from $\mathcal{B}$ to $\mathcal{L}$. This map is written as $\xi \rightarrow r^{-1 / 2} \mathcal{T}(\xi)$. Meanwhile what is denoted by $\mathcal{T}$ is decomposed as $\mathcal{T}(\xi)=\mathcal{T}_{0}+\mathcal{T}_{1} \xi+\mathcal{T}_{2}(\xi)$ where $\mathcal{T}_{0}=\mathcal{T}(0)$, where $\mathcal{T}_{1}$ is a linear map from $\mathcal{K}$ to $\mathcal{L}$ and where $\mathcal{T}_{2}$ is, by definition, the rest of $\mathcal{T}$. Suppose that $\mathcal{T}_{1}$ is invertible on the kernel of the map $\mathfrak{q}$. Use $\mathcal{T}_{1}^{-1}$ to denote the inverse of the restriction of $\mathcal{T}_{1}$ to the kernel of $\mathfrak{q}$. Then a solution to (7-1) in the kernel of $\mathfrak{q}$ is neither more nor less than a fixed point to a map from the ball $\mathcal{B} \cap \operatorname{kernel}(\mathfrak{q})$ to itself that has the form

$$
\xi \rightarrow-\left(\mathcal{T}_{1}\right)^{-1}\left(\mathcal{T}_{0}+\mathcal{T}_{2}(\xi)\right) .
$$

The contraction mapping theorem can be used to find a unique small normed fixed point to this map given suitable bounds on the norm of $\mathcal{T}_{0}$, the operator norm of $\left(\mathcal{T}_{1}^{-1}\right)$, and the size of both $\mathcal{T}_{2}$ and its differential. A slight generalization of this sort of contraction mapping argument gives a unique solution to (7-1) that maps via $\mathfrak{q}$ to any given small normed element in $V_{0}$. The next proposition describes the salient features of $\mathcal{T}_{0}, \mathcal{T}_{1}$, $\mathcal{T}_{2}$, and $\mathfrak{q}$. 
Proposition 7.2 There is a constant $\kappa>100$ and a finite dimensional, normed vector space, $V_{0}$, with the following significance: Make the constructions that lead to the equation in (7-1) using $R \geq \kappa, r \geq \kappa$, and with the radius of Proposition 6.4's ball $\mathcal{B}$ less than $\kappa^{-1}$. Then the left hand side of (7-1) defines a smooth map from $\mathcal{B}$ to $\mathcal{L}$. Write this map as $r^{-1 / 2} \mathcal{T}$. There is a decomposition of $\mathcal{T}$ as $\mathcal{T}_{0}+\mathcal{T}_{1}+\mathcal{T}_{2}$ where $\mathcal{T}_{0}$, $\mathcal{T}_{1}$ and $\mathcal{T}_{2}$ obey the following:

- $\mathcal{T}_{0}=\mathcal{T}(0)$ obeys $\left\|\mathcal{T}_{0}\right\|_{\mathcal{L}} \leq \kappa r^{-1 / 2+8 \sigma}$.

- There is a surjective map $q: \mathcal{K} \rightarrow \mathbb{V}_{0}$ with $|q(\xi)| \leq \kappa\|\xi\|_{\mathcal{L}^{2}}$ and such that

(a) $\mathcal{T}_{1}$ maps the kernel of $q$ isomorphically to $\mathcal{L}$.

(b) $\left\|\mathcal{T}_{1}(\xi)\right\|_{\mathcal{L}}+|q(\xi)| \geq \kappa^{-1}\|\xi\|_{\mathcal{K}}$.

In addition,

(c) $\mathcal{T}_{1}(\xi)\left\|_{\mathcal{L}^{2}}+|q(\xi)| \geq \kappa^{-1}\right\| \xi \|_{\mathcal{K}_{1}^{2}}$.

- $\left\|\mathcal{T}_{2}(\xi)\right\|_{\mathcal{L}} \leq \kappa\left(r^{-1 / 4+8 \sigma}+\|\xi\|_{\mathcal{K}}\right)\|\xi\|_{\mathcal{K}}$.

- In addition, $\left\|\mathcal{T}_{2}(\xi)-\mathcal{T}_{2}\left(\xi^{\prime}\right)\right\|_{2} \leq \kappa\left(r^{-1 / 4+8 \sigma}+\|\xi\|_{\mathcal{K}}+\left\|\xi^{\prime}\right\|_{\mathcal{K}}\right)\left\|\xi-\xi^{\prime}\right\|_{\mathcal{K}}$.

By way of a reminder, $\|\cdot\|_{\mathcal{K}_{1}^{2}}$ is defined by replacing each occurrence of $\|\cdot\|_{\mathcal{K}}$ in (5-13) by the (2-27)'s norm $\|\cdot\|_{\mathcal{K}_{1}^{2}}$. Note that (c) of the second bullet is not used below in the proof of Proposition 7.1. Proposition 7.2 is proved in Sections 7.b-7.i.

Note for reference later that Constraint 4 in Section 4.b enters only in the proof of the second bullet; in particular, it is invoked only in Section 7.g and Section 7.h to establish that $\mathcal{T}_{1}$ is surjective.

Proposition 7.1 is a corollary to Proposition 7.2. To elaborate, fix $\varepsilon>0$ such that (7-1) is defined on the ball $\mathcal{B} \subset \mathcal{K}$ centered at the origin of radius $\frac{1}{4} \varepsilon$. Use $\mathcal{F}$ to denote the linear map $\left(\mathcal{T}_{1}, q\right): \mathcal{K} \rightarrow \mathcal{L} \times V_{0}$. It is a consequence of the second bullet that $\mathcal{F}$ is invertible, and that the norm of its inverse is bounded by $c_{0}$. In this regard, the norm is defined using the norm $\|\cdot\|_{\mathcal{K}}$ on $\mathcal{K}$ and the product norm on $\mathcal{L} \times V_{0}$.

Fix $\lambda \in \mathbb{V}_{0}$ and let $x_{\lambda}: \mathcal{B} \rightarrow \mathcal{K}$ denote the map $\xi \rightarrow-(\mathcal{F})^{-1}\left(\mathcal{T}_{0}+\mathcal{T}_{2}(\xi),-\lambda\right)$. The map $X_{\lambda}$ obeys $\left\|X_{\lambda}(\xi)\right\|_{\mathcal{K}} \leq c_{0}\left(r^{-1 / 2+16 \sigma}+\|\xi\|_{\mathcal{K}}^{2}+|\lambda|\right)$. It is a consequence of the third and fourth bullets of Proposition 7.2 that there exists $c_{0}>4+\varepsilon^{-1}$ with the following significance: If $r \geq c_{0}$ and if $|\lambda| \leq c_{0}^{-3}$, then $X_{\lambda}$ maps the radius $c_{0}^{-1}$ ball in $\mathcal{B}$ to itself as a contraction mapping. Thus, it has a unique fixed point in this ball. By construction, the fixed point, $\xi_{\lambda}$, obeys the equations $\mathcal{T}\left(\xi_{\lambda}\right)=0$ and $q\left(\xi_{\lambda}\right)=\lambda$. Moreover, its norm is bounded by $c_{0}\left(r^{-1 / 2+8 \sigma}+|\lambda|\right)$. The fact that the assignment of $\xi_{\lambda}$ to $\lambda$ defines a smooth map follows in a standard manner by differentiating the fixed point equation. 


\section{7.b Proof of Proposition 7.2: Part 1}

This subsection proves that left hand side of (7-1) is in $\mathcal{L}$ when $\xi \in \mathcal{B}$ and explains why the resulting map is Lipschitz. The existence of the higher derivatives of the map use the same sort of arguments that are used to prove that the map is Lipschitz. This understood, no more will be said about these higher derivatives.

As noted above, it follows from the fourth point in Proposition 6.4 that the left hand side defines a smooth map from $\mathcal{B}$ to $\mathcal{L}^{2}$. This understood, there are two outstanding questions:

Question 1 Is the contribution to the $\mathcal{L}$ norm from the far right term in (2-29) finite on the left hand side of (7-1) as defined by any given $\xi \in \mathcal{B}$; and if so, does this contribution have a $\xi$-independent upper bound?

Question 2 Is this same contribution to the $\mathcal{L}$ norm finite on the derivative of the left hand side of (7-1) at $\xi \in \mathcal{B}$ along any unit length vector $\xi^{\prime} \in \mathcal{K}$; and if so, does this contribution have an upper bound that is independent of $\xi$ and $\xi^{\prime}$ ?

Both these questions are answered affirmatively in what follows.

The subsequent arguments require a lemma that strengthens the fifth point in Proposition 6.4. To set the stage, fix $\rho>0$ and let $D_{\rho}$ denote a disk of radius $\rho$ as described in Part 2 of the digression that follows Lemma 6.5. Define $\hat{\chi}$ as done in this same part of the digression. When $\mathfrak{f} \in \mathbb{H}$, set

$$
n_{\mathfrak{f}}(\rho)=\int_{z \in D_{\rho}} \int_{\pi^{-1}(z)} \hat{\chi}^{2} e^{-\sqrt{r} \operatorname{dist}(\Sigma, \cdot) / 200}|\nabla \mathfrak{f}|^{2} .
$$

Here is the promised lemma.

Lemma 7.3 There exists $\kappa>1$ with the following significance: Assume that $R \geq \kappa$ that $r \geq \kappa$, that $v<\kappa^{-1}$ and that the norm that defines Proposition 6.4's ball $\mathcal{B}$ is less than $\kappa^{-1}$. Fix $\xi \in \mathcal{B}$ and define $\mathfrak{q}$ from $\xi$ as instructed by Proposition 6.4. If $\rho \leq r^{-1 / 2}$ then any $D_{\rho}$ version of $\mathfrak{n}_{\mathfrak{q}}(\rho)$ is bounded by $\kappa r^{-1}\left(r^{-1 / 2+8 \sigma}+\|\xi\|_{\mathcal{K}}\right)^{2} \rho^{v}$. Let $\xi^{\prime} \in \mathcal{K}$ have unit norm and define $\mathfrak{q}^{\prime}$ to be the derivative of $\mathfrak{q}$ at $\xi$ in the direction of $\xi^{\prime}$. If $\rho \leq r^{-1 / 2}$, then any $D_{\rho}$ version of $\mathfrak{n}_{\mathfrak{q}^{\prime}}(\rho)$ is bounded by $\kappa r^{-1} \rho^{v}$.

This lemma is proved in the next subsection. Assume it for the moment. Given Lemma 7.3, the proof that Question 1 and Question 2 have affirmative answers has seven steps. 
Step 1 To review what is involved keep in mind that the left hand side of (7-1) defines an element in $\mathcal{L}^{2}$ and so can be written as $\left.\lambda=\left(\left(\lambda_{C}\right)_{C \in \mathcal{C}},\left(\lambda_{\gamma-}\right)_{\gamma \in \Xi_{\Sigma_{-}}}\right),\left(\lambda_{\gamma+}\right)_{\gamma \in \Xi_{\Sigma+}}\right)$. The $\mathcal{L}$ norm of $\lambda$ is defined using the $\mathcal{L}^{2}$ norm of $\lambda_{C}$ over $C-\left(\bigcup_{\mathcal{E} \in \mathcal{E}_{C}} \mathcal{E}_{2 R}\right)$, the $L^{2}$ norm of each $\lambda_{\gamma-}$ over various regions in $(-\infty,-R] \times S^{1}$, and the $L^{2}$ norm of each $\lambda_{\gamma+}$ over various regions in $[R, \infty) \times S^{1}$. A given disk $D_{\rho}$ from Part 2 of the digression subsequent to Lemma 6.5 is assumed implicitly to lie in either some $C \in \mathcal{C}$ version of $C-\left(\bigcup_{\mathcal{E} \in \mathcal{E}_{C}} \mathcal{E}_{2 R}\right)$, or in some $\gamma \in \Xi_{\Sigma-}$ version of $(-\infty,-R] \times S^{1}$, or in some $\gamma \in \Xi_{\Sigma+}$ version of $[R, \infty) \times S^{1}$.

Step 2 Fix $\rho \in(0,1 / 100)$ and let $D_{\rho}$ be as described above. Define $\mathcal{A}_{1}$ and $\mathcal{A}_{2}$ as in (6-67). The contribution to the square of $\left\|\Pi_{\xi} \mathfrak{D} \mathfrak{q}\right\|_{L^{2}}$ from $D_{\rho}$ is bounded by $c_{0}\left(\mathcal{A}_{1}+\mathcal{A}_{2}\right)$. It follows as a consequence that

$$
\left\|\Pi_{\xi} \mathfrak{D q}\right\|_{L^{2}} \leq c_{0} r^{-1 / 2}\left(r^{-1 / 2+8 \sigma}+\|\xi\|_{\mathcal{K}}\right)^{2} .
$$

The norm that defines $\mathcal{L}$ also requires a $\rho$ independent bound for $\rho^{-v} \int_{D_{\rho}}\left|\Pi_{\xi} \mathfrak{D q}\right|^{2}$. Such a bound follows from the assertion that

$$
\int_{D_{\rho}}\left|\Pi_{\xi} \mathfrak{D q}\right|^{2} \leq c_{0} r^{-1}\left(r^{-1 / 2+8 \sigma}+\|\xi\|_{\mathcal{K}_{1}^{2}}\right)^{2}\left(r^{-1 / 2+8 \sigma}+\|\xi\|_{\mathcal{K}}\right)^{2} \rho^{v} .
$$

To derive (7-5), use Step 2 of the proof of Lemma 6.5 to see that the left hand side is bounded by $c_{0}\left(\mathcal{A}_{1}+\mathcal{A}_{2}\right)$. A bound for $\mathcal{A}_{1}$ is given in (6-68) with

$$
\mathfrak{m}_{\mathbb{L}}=r^{-1}\left(r^{-1 / 2+8 \sigma}+\|\xi\|_{\mathcal{K}_{1}^{2}}\right)^{2} .
$$

A bound for $\mathcal{A}_{2}$ is given in (6-69) using this same $\mathfrak{m}_{\mathbb{L}}$ for the case $\rho \geq r^{-1 / 2-v}$. A bound for $\mathcal{A}_{2}$ in the case $\rho<r^{-1 / 2}$ that implies (7-5) follows from Lemma 7.3.

Step 3 This step bounds the $\mathcal{L}$-norms of $r^{1 / 2} \Pi_{\xi}(\mathfrak{h} * \mathfrak{q})$ and $r^{1 / 2} \Pi_{\xi}(\mathfrak{q} * \mathfrak{q})$. To this end, note that the square of the $L^{2}$ norm of $r^{1 / 2} \Pi_{\xi}(\mathfrak{h} * \mathfrak{q})$ over the disk $D_{\rho}$ is bounded by $c_{0} \mathcal{A}_{3}$ with the latter defined as in (6-70). Granted this, it follows from the conclusions of Step 3 in the proof of Lemma 6.5 that

$$
r^{1 / 2}\left\|\Pi_{\xi}(\mathfrak{h} * \mathfrak{q})\right\|_{\mathcal{L}} \leq c_{0} r^{-1 / 2}\left(r^{-1 / 2+8 \sigma}+\|\xi\|_{\mathcal{K}}\right) r^{-1 / 16} .
$$

Meanwhile, the square of the $L^{2}$ norm of $r^{1 / 2}\left\|\Pi_{\xi}(\mathfrak{q} * \mathfrak{q})\right\|_{\mathcal{L}}$ over $D_{\rho}$ is bounded by $c_{0} \mathcal{A}_{4}$ with the latter given by the $\mathfrak{f}=\mathfrak{q}$ version of (6-74). There are two cases to consider. The first is when $\rho>r^{-1 / 2}$. As explained momentarily, the Sobolev inequality depicted in (6-64) bounds $\mathcal{A}_{4}$ by $c_{0} r \rho^{v}\|\mathfrak{q}\|_{\mathbb{H}}^{2}\|\mathfrak{q}\|_{*}^{2}$. As a consequence, Points 3 and 4 of Proposition 6.4 find that $\mathcal{A}_{4} \leq c_{0} r^{-1}\left(r^{-1 / 2+8 \sigma}+\|\xi\|_{\mathcal{K}_{1}^{2}}\right)^{2}\left(r^{-1 / 2+8 \sigma}+\|\xi\|_{\mathcal{K}}\right)^{2} \rho^{v}$ when $\rho>r^{-1 / 2}$. 
To say more about the derivation of this $\rho>r^{-1 / 2}$ bound for $\mathcal{A}_{4}$, note that Hölder's inequality bounds $\mathcal{A}_{4}$ by $c_{0}$ times the integral of $r \hat{\chi}^{2}|\mathfrak{q}|^{4} e^{-\sqrt{r} \operatorname{dist}(\Sigma, \cdot) / c_{0}}$ over $\pi^{-1}\left(D_{2 \rho}\right)$. To bound the latter, fix an identification of $\pi^{-1}\left(D_{\rho}\right)$ with $D_{\rho} \times \mathbb{C}$. Let $\Lambda \subset \mathbb{C}$ denote the square lattice $\frac{1}{2} \rho \mathbb{Z}^{2}$. Given $u \in \Lambda$, let $D(u)$ denote the disk with radius $2 \rho$ centered on $u$. Introduce $\Delta(u)=\inf _{x \in D \times D(u)} \operatorname{dist}(x, \Sigma)$. Let $\Lambda^{\prime} \subset \Lambda$ denote the set of elements $u$ such that $D_{\rho} \times D(u)$ intersects the support of $\hat{\chi}$. Fix $u \in \Lambda^{\prime}$ and use the Sobolev inequalities in (6-3) and (6-64) to bound the integral of $r \hat{\chi}^{2}|\mathfrak{q}|^{4} e^{-\sqrt{r} \operatorname{dist}(\Sigma, \cdot) / c_{0}}$ over $D_{2 \rho} \times D(u)$ by $r \rho^{v}\|\mathfrak{q}\|_{\mathbb{H}}^{2}\|\mathfrak{q}\|_{*}^{2} e^{-\sqrt{r} \Delta(u) / c_{0}}$. As a consequence, the $\rho>r^{-1 / 2}$ version of $\mathcal{A}_{4}$ is no greater than $c_{0} r \rho^{v}\|\mathfrak{q}\|_{\mathbb{H}}^{2}\|\mathfrak{q}\|_{*}^{2} \sum_{u \in \Lambda^{\prime}} e^{-\sqrt{r} \Delta(u) / c_{0}}$. The sum here converges with bound $c_{0}$, so the $\rho>r^{-1 / 2}$ version of $\mathcal{A}_{4}$ is no greater than $r \rho^{v}\|\mathfrak{q}\|_{\mathbb{H}}^{2}\|\mathfrak{q}\|_{*}^{2}$.

To obtain a bound for when $\rho \leq r^{-1 / 2}$ let $D$ now denote the disk of radius $2 r^{-1 / 2}$ that is concentric with $D_{\rho}$. Now write $\mathcal{A}_{4}$ as $c_{0} r \int_{D_{2 \rho}} f^{2}$ where $f$ is the function on $D$ given by $f(\cdot)=\int_{\pi^{-1}(\cdot)} \theta_{r}|\mathfrak{q}|^{2}$. As it turns out, this function is in $L_{1}^{4 / 3}$ on $D$ with norm bounded by $c_{0} r^{1 / 4} r^{-v / 4}\|\mathfrak{q}\|_{\mathbb{H}}\|\mathfrak{q}\|_{*}$. To see why this is, use Hölder's inequality to see that

$$
|d f| \leq c_{0} f^{1 / 2}\left(\int_{\pi^{-1}(z)} \theta_{r}\left|\nabla^{*} \mathfrak{q}\right|^{2}\right)^{1 / 2},
$$

and then use Hölder's inequality a second time to obtain

$$
\int_{D}|d f|^{4 / 3} \leq\left(\int_{D} f^{2}\right)^{1 / 3}\left(\int_{D} \int_{\pi^{-1}(z)} \theta_{r}\left|\nabla^{*} \mathfrak{q}\right|^{2}\right)^{2 / 3} .
$$

Meanwhile, Hölder's inequality also bounds the square of the $L^{2}$ norm over $D$ of $f$ by

$$
c_{0} \int_{D} \int_{\pi^{-1}(z)} \hat{\chi}^{2} e^{-\sqrt{r} \operatorname{dist}(\Sigma, \cdot) / c_{0}}|\mathfrak{q}|^{4} .
$$

To bound the latter, fix an identification of $\pi^{-1}(D)$ with $D \times \mathbb{C}$. Let $\Lambda \subset \mathbb{C}$ denote the square lattice $\frac{1}{2} r^{-1 / 2} \mathbb{Z}^{2}$. Let $D(u)$ for $u \in \Lambda$ denote the disk with radius $2 r^{-1 / 2}$ centered on $u$. It follows now that (7-9) is bounded by

$$
\begin{aligned}
& c_{0} \sum_{u \in \Lambda}\left(e^{\sqrt{r} \operatorname{dist}(u, \Sigma) / c_{0}} \int_{(D \times D(u))}|\mathfrak{q}|^{4}\right) \\
& \quad \leq c_{0}\left(\sum_{u \in \Lambda} e^{-\sqrt{r} \operatorname{dist}(u, \Sigma) / c_{0}}\right)\|\mathfrak{q}\|_{\mathbb{H}}^{2}\|\mathfrak{q}\|_{*}^{2} r^{-v / 2} .
\end{aligned}
$$

Note that the sum that appears is bounded by $c_{0}$. A similar use of the lattice $\Lambda$ bounds the $L^{2}$ norm of $\nabla^{*} \mathfrak{q}$ over the domain of the right most integral in (7-8) by $\left(r^{-v / 4}\|\mathfrak{q}\|_{\mathbb{H}}\|\mathfrak{q}\|_{*}\right)^{1 / 2}$. All together, this bounds the right hand side of (7-8) by 
$c_{0} r^{1 / 3}\left(r^{-v / 4}\|\mathfrak{q}\|_{\mathbb{H}}\|\mathfrak{q}\|_{*}\right)^{4 / 3}$. (The factor $r^{1 / 3}$ here accounts for the factor $\theta_{r}$ that appears in the right most integral of (7-8); this is because $\left|\theta_{r}\right| \leq c_{0} r^{1 / 2}$.) To continue, note that there is a borderline Sobolev embedding that sends $L_{1}^{4 / 3}(D)$ into $L^{4}(D)$. Use this Sobolev inequality after Hölder's inequality to obtain the chain

$$
\begin{aligned}
\int_{D_{2 \rho}} f^{2} & \leq c_{0} \rho\|\mathfrak{f}\|_{4}^{2} \\
& \leq c_{0} \rho\|\mathfrak{f}\|_{L_{1}^{4 / 3}}^{2} \\
& \leq c_{0} \rho r^{1 / 2} r^{-v / 2}\|\mathfrak{q}\|_{\mathbb{H}}^{2}\|\mathfrak{q}\|_{*}^{2} .
\end{aligned}
$$

As an aside for use in the proof of the upcoming Lemma 7.4, remark that almost the same arguments bound the $\rho \leq r^{-1 / 2}$ versions of $\int_{D_{2} \rho} f^{2}$ by $c_{0} \rho r^{1 / 2} r^{v}\|\mathfrak{q}\|_{*}^{4}$. Indeed, such a bound arises by using $c_{0} r^{-v / 4}\|\mathfrak{q}\|_{*}$ in lieu of $c_{0}\left(r^{-v / 4}\|\mathfrak{q}\|_{*}\|\mathfrak{q}\|_{\mathbb{H}}\right)^{1 / 2}$ to bound the $L^{4}$ norm of $\mathfrak{q}$ and the $L^{2}$ norm of $\nabla^{*} \mathfrak{q}$ over $D \times D(u)$.

To continue, note that (7-11) together with Points 3 and 4 in Proposition 6.4 imply that the $\rho \leq r^{-1 / 2}$ version of $\mathcal{A}_{4}$ is no greater than $c_{0} r^{-1}\left(r^{-1 / 2+8 \sigma}+\|\xi\|_{\mathcal{K}_{1}^{2}}\right)^{2}$ $\times\left(r^{-1 / 2+8 \sigma}+\|\xi\|_{\mathcal{K}}\right)^{2} \rho^{v}$. Given these $\rho>r^{-1 / 2}$ and $\rho<r^{-1 / 2}$ bounds on $\mathcal{A}_{4}$, it follows that

$$
r^{1 / 2}\left\|\Pi_{\xi}(\mathfrak{q} * \mathfrak{q})\right\|_{\mathcal{L}} \leq c_{0} r^{-1 / 2}\left(r^{-1 / 2+8 \sigma}+\|\xi\|_{\mathcal{K}_{1}^{2}}\right)\left(r^{-1 / 2+8 \sigma}+\|\xi\|_{\mathcal{K}}\right) .
$$

Step 4 The $\mathcal{L}$ norm of $\Pi_{\xi}\left(\mathfrak{v}-\mathfrak{v}_{\mathfrak{h}}\right)$ is finite apriori given that $\xi \in \mathcal{K}$ and given what is said about $\mathfrak{b}_{0}, \mathfrak{b}_{ \pm},\left\{\mathfrak{b}_{C}\right\}_{C \in \mathcal{C}},\left\{\mathfrak{b}_{\gamma-}\right\}_{\gamma \in \Xi_{\Sigma-}},\left\{\mathfrak{b}_{\gamma+}\right\}_{\gamma \in \Xi_{\Sigma+}}$ in the proof of Lemma 6.3. More is said in the upcoming Section 7.d about $\Pi_{\xi}\left(\mathfrak{v}-\mathfrak{v}_{\mathfrak{h}}\right)$.

Step 5 View $\mathcal{T}$ momentarily as a map to the space $\mathcal{L}^{2}$. As such, it is smooth; this a consequence of the fourth bullet of Proposition 6.4. To see if $\mathcal{T}$ is a Lipschitz map to $\mathcal{L}$, fix $\xi \in \mathcal{B}$ and let $\xi^{\prime} \in \mathcal{K}$ denote a unit length vector. The directional derivative of $\mathcal{T}$ (viewed as a map to $\mathcal{L}^{2}$ ) in the direction of $\xi^{\prime}$ has two parts. To describe these two parts, write the components of $\mathcal{T}$ as $\left(\left(\mathcal{T}_{C}\right)_{C \in \mathcal{C}},\left(\mathcal{T}_{\gamma-}\right)_{\gamma \in \Xi_{\Sigma-}},\left(\mathcal{T}_{\gamma+}\right)_{\gamma \in \Xi_{\Sigma+}}\right)$. The component $\mathcal{T}_{C}$ on the $|s| \leq R$ part of $C$ has the same form as what is written on the left hand side of (6-57) with $\mathfrak{f}_{0}$ defined by $\mathfrak{f}=\mathfrak{D} \mathfrak{q}+r^{1 / 2} \mathfrak{q} * \mathfrak{q}+2 r^{1 / 2} \mathfrak{h} * \mathfrak{q}-\left(\mathfrak{v}-\mathfrak{v}_{\mathfrak{h}}\right)$. This understood, the differential of $\mathcal{T}_{C}$ here has two contributions. The first is from the differential of $\mathfrak{f}$, and the second comes from the differential of what is written as $\left(1+z_{p}\right) \theta_{1 \xi, r}^{\dagger}$ in (6-57). The differentials of the other components of $\mathcal{T}$ have the analogous two contributions. The contribution to the differential of $\mathcal{T}$ that comes from the change in $\mathfrak{D} \mathfrak{q}+r^{1 / 2} \mathfrak{q} * \mathfrak{q}+2 r^{1 / 2} \mathfrak{h} * \mathfrak{q}-\left(\mathfrak{v}-\mathfrak{v}_{\mathfrak{h}}\right)$ can be written as

$$
\mathcal{T}^{\prime}=r^{1 / 2} \Pi_{\xi}\left(\mathfrak{D} \mathfrak{q}^{\prime}+2 r^{1 / 2} \mathfrak{q} * \mathfrak{q}^{\prime}+2 r^{1 / 2} \mathfrak{h} * \mathfrak{q}^{\prime}+2 r^{1 / 2} \mathfrak{h}^{\prime} * \mathfrak{q}-\left(\mathfrak{v}^{\prime}-\mathfrak{v}_{\mathfrak{h}}^{\prime}\right)\right),
$$


where the primes denote the directional derivative of the indicated term in the $\xi^{\prime}$ direction. The second contribution to $\mathcal{T}$ 's differential has finite $\mathcal{L}$ norm with bound $c_{0}\|\mathcal{T}\|_{\mathcal{L}}$.

Granted the preceding, it follows that $\mathcal{T}$ defines a Lipschitz map to $\mathcal{L}$ provided that the contribution to its directional derivative from $\mathcal{T}^{\prime}$ has finite $\mathcal{L}$ norm. To see that such is the case, write $\mathfrak{q}^{\prime}=\Pi_{\xi} \mathfrak{q}^{\prime}+\mathfrak{q}^{\perp}$. Noting that $\Pi_{\xi} \mathfrak{q}^{\prime}=-\Pi_{\xi}^{\prime} \mathfrak{q}$, write $\mathcal{T}^{\prime}$ as a sum of two terms. The first is $-r^{1 / 2} \Pi_{\xi} D \Pi_{\xi}^{\prime} \mathfrak{q}$ and the second is

$$
\mathcal{T}_{*}=r^{1 / 2} \Pi_{\xi}\left(\mathfrak{D} \mathfrak{q}^{\perp}+2 r^{1 / 2} \mathfrak{q} * \mathfrak{q}^{\prime}+2 r^{1 / 2} \mathfrak{h} * \mathfrak{q}^{\prime}+\mathfrak{h}^{\prime} * \mathfrak{q}-\left(\mathfrak{v}^{\prime}-\mathfrak{v}_{\mathfrak{h}}^{\prime}\right)\right)
$$

Step 6 This step considers the $\mathcal{L}$ norm of $-\Pi_{\xi} \mathfrak{D} \Pi_{\xi \mathfrak{q}}^{\prime} \mathfrak{q}$. The $L^{2}$ norm of this term over a disk $D_{\rho}$ is bounded by

$$
\begin{aligned}
c_{0} \int_{z \in D_{2 \rho}}\left(\left(|\nabla \xi|^{2}+\left|\nabla \xi^{\prime}\right|^{2}+1\right)\left(\int_{\pi^{-1}(z)} \theta_{r}|\mathfrak{q}|\right)^{2}\right) & \\
& +c_{0} \int_{z \in D_{2 \rho}}\left(\int_{\pi^{-1}(z)} \theta_{r}|\nabla \mathfrak{q}|\right)^{2} .
\end{aligned}
$$

This follows using (2-11) and (2-12).

It follows from [3, Lemmas 2.9 and 5.4.1] that the left most integral in (7-15) is bounded by $c_{0} \rho^{v}\|\mathfrak{q}\|_{\mathbb{H}}^{2} \leq c_{0} r^{-1}\left(r^{-1 / 2+8 \sigma}+\|\xi\|_{\mathcal{K}}\right)^{2} \rho^{v}$. To bound the right most integral when $\rho>r^{-1 / 2}$, fix an identification of $\pi^{-1}\left(D_{2 \rho}\right)$ with $D_{2 \rho} \times \mathbb{C}$. Let $\Lambda \subset \mathbb{C}$ denote the square lattice $\frac{1}{2} \rho \mathbb{Z}^{2}$. Let $D_{2 \rho}(u)$ for $u \in \Lambda$ denote the disk with radius $2 \rho$ centered on $u$. It follows now that the right most integral in (7-15) is bounded when $\rho \geq r^{-1 / 2}$ by

$$
\begin{aligned}
c_{0} \sum_{u \in \Lambda}\left(e^{-\sqrt{r} \operatorname{dist}(\Sigma, u) / c_{0}} \int_{\left(D_{2 \rho} \times D_{2 \rho}(u)\right)}\right. & \left.\operatorname{support(\hat {\chi })}|\nabla \mathfrak{q}|^{2}\right) \\
& \leq c_{0}\left(\sum_{u \in \Lambda} e^{-\sqrt{r} \operatorname{dist}(\Sigma, u) / c_{0}}\right)\|\mathfrak{q}\|_{*}^{2} \rho^{v} .
\end{aligned}
$$

Since $\rho \geq r^{-1 / 2}$, the sum on the far right in (7-16) is bounded by $c_{0}$. Thus the far right hand integral in (7-15) is bounded by $c_{0} r^{-1}\left(r^{-1 / 2+8 \sigma}+\|\xi\|_{\mathcal{K}}\right)^{2} \rho^{v}$ when $\rho \geq r^{-1 / 2}$. When $\rho \leq r^{-1 / 2}$, the integral on the far right in (7-15) is bounded by $\mathfrak{n}_{\mathfrak{q}}(\rho)$ and thus guaranteed by Lemma 7.3 to be less than $c_{0} r^{-1}\left(r^{-1 / 2+8 \sigma}+\|\xi\|_{\mathcal{K}}\right)^{2} \rho^{v}$.

Step 7 This step considers the $\mathcal{L}$ norms of the various terms that contribute to $\mathcal{T}_{*}$. Consider first the square of the $L^{2}$ norm of $\Pi_{\xi} \mathfrak{D} \mathfrak{q}^{\prime}$ over $D_{\rho}$. The corresponding integral is bounded by $c_{0}\left(\mathcal{A}_{1}^{\prime}+\mathcal{A}_{2}^{\prime}\right)$ where $\mathcal{A}_{1}^{\prime}$ and $\mathcal{A}_{2}^{\prime}$ are defined by replacing $q$ 
with $\mathfrak{q}^{\prime}$ in (6-67). This understood, the analysis done in Step 2 can be repeated with only notational modifications to see that

$$
\left\|\Pi_{\xi} \mathfrak{D} \mathfrak{q}^{\prime}\right\|_{\mathcal{L}} \leq c_{0} r^{-1 / 2}\left(r^{-1 / 4+8 \sigma}+\|\xi\|_{\mathcal{K}}\right) .
$$

The square of the $L^{2}$ norm of $r^{1 / 2} \Pi_{\xi}\left(\mathfrak{h} * \mathfrak{q}^{\prime}\right)$ over the disk $D_{\rho}$ is bounded by $c_{0} \mathcal{A}_{3}^{\prime}$ where $\mathcal{A}_{3}^{\prime}$ is defined by replacing $\mathfrak{q}$ with $\mathfrak{q}^{\prime}$ in (6-70). This understood, the arguments that lead to (6-72) and (6-73) can be repeated with only notational modifications to see that $r^{1 / 2}\left\|\mathfrak{h} * \mathfrak{q}^{\prime}\right\|_{\mathcal{L}}$ is also bounded by what is written on the right hand side of (7-17).

The square of the $L^{2}$ norm of $r^{1 / 2} \Pi_{\xi}\left(\mathfrak{q} * \mathfrak{q}^{\prime}\right)$ over $D_{\rho}$ is bounded by $c_{0} \mathcal{A}_{4}^{\prime}$ where $\mathcal{A}_{4}^{\prime}$ is the $\mathfrak{f}=\mathfrak{q}^{\prime}$ version of (6-74). Granted this, the arguments in Step 3 that lead to (7-12) can be repeated with only cosmetic changes to prove that $r^{1 / 2}\left\|\mathfrak{q} * \mathfrak{q}^{\prime}\right\|_{\mathcal{L}}$ is also bounded by the expression on the right hand side of (7-17).

Consider next the $\mathcal{L}$ norm of $r^{1 / 2} \mathfrak{h}^{\prime} * \mathfrak{q}$. The square of the $L^{2}$ norm of the latter over the disk $D_{\rho}$ is bounded by $c_{0} \mathcal{A}_{3}^{\prime \prime}$ where $\mathcal{A}_{3}^{\prime \prime}$ is defined by replacing $\mathfrak{h}$ in (6-70) with $\mathfrak{h}^{\prime}$. As can be seen in the proof of Lemma $6.3, \mathfrak{h}$ depends on $\xi$ only through the various $\mathfrak{b}_{\gamma \pm}$ that are defined in the Part 5 of this same proof. In particular, what is defined there as $\mathfrak{p}_{\xi}$ can be differentiated, and its derivative along $\xi^{\prime}$ also obeys (6-42). As a

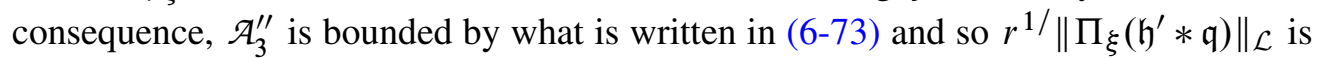
also bounded by what is written on the right hand side of (7-17).

The fact that $\Pi_{\xi}\left(\mathfrak{v}^{\prime}-\mathfrak{v}_{\mathfrak{h}}^{\prime}\right)$ has finite $\mathcal{L}$ norm is a consequence of the inclusion of the $\|\cdot\|_{\mathcal{K}_{1}^{2}}$ norm in (2-27)'s definition of $\|\cdot\|_{\mathcal{K}}$. More is said about this term in Section 7.e.

\section{7.c Proof of Lemma 7.3}

The desired bounds on $\mathfrak{n}_{\mathfrak{q}}(\rho)$ and $\mathfrak{n}_{\mathfrak{q}^{\prime}}(\rho)$ in this case are seen to follow from the next lemma. To set the stage for the lemma, suppose that $\mathfrak{f} \in \mathbb{H}$ and $\mathfrak{u} \in \mathbb{L} \perp$ have been given. It is assumed in what follows that there exists $\mathfrak{m}_{*}$ such that

$$
\int_{z \in D_{\rho}} \int_{\pi^{-1}(z)} \hat{\chi} e^{-\sqrt{r} \operatorname{dist}(\Sigma, \cdot) / 200}|\mathfrak{u}|^{2} \leq \mathfrak{m}_{*} \rho^{v}
$$

for each $\rho \leq T^{-1} r^{-1 / 2}$ and for each disk $D_{\rho}$ as described in Part 2 of the digression subsequent to Lemma 6.5.

Lemma 7.4 There exists $\kappa \geq 1$ with the following significance: Suppose that $r \geq \kappa$, that $v \leq 1 / \kappa$ and that $\xi \in \mathcal{K}_{*}$ has $\|\xi\|_{\infty} \leq \kappa^{-1}$. Suppose that $\mathfrak{f} \in \mathbb{H}$ obeys $\|\mathfrak{f}\|_{\mathbb{H}} \leq$ $\kappa^{-1} r^{-1 / 2}$ and that $\mathfrak{u} \in \mathbb{L}$ obeys (6-56). Let $\mathfrak{q} \in \mathbb{H}_{\xi}^{\perp}$ denote a solution to (6-55) with $\|\mathfrak{q}\|_{*}$ finite. Then any $\rho \leq r^{-1 / 2}$ and $D_{\rho}$ version of $\mathfrak{n}_{\mathfrak{q}}(\rho)$ is bounded by $\kappa\left(\mathfrak{m}_{*}+\|\mathfrak{q}\|_{*}^{2}+\mathfrak{n}_{\mathfrak{q}}\left(r^{-1 / 2}\right) r^{v / 2}\right) \rho^{v}$. 
This lemma is proved momentarily.

To see how to obtain Lemma 7.3, consider first the asserted bound on $\mathfrak{n}_{\mathfrak{q}}(\rho)$. In this case, $\mathfrak{q}$ obeys the version of (6-55) with $\mathfrak{f}=\mathfrak{q}$ and $u=\left(1-\Pi_{\xi}\right)\left(\mathfrak{v}-\mathfrak{v}_{\mathfrak{h}}\right)$. The required bound on $\|\mathfrak{f}\|_{\mathbb{H}}$ follows from Proposition 6.4 if $r \geq c_{0}$ and $\|\xi\|_{\mathcal{K}} \leq 1 / c_{0}$. Given that $\|\cdot\|_{\mathcal{K}}$ as given in (2-27) contains the $\|\cdot\|_{\mathcal{K}_{*}}$ norm, it follows from Parts 3-6 of the proof of Lemma 6.3 that $u$ obeys (7-18) with $\mathfrak{m}_{*}=c_{0} r^{-1}\left(r^{-1 / 2+8 \sigma}+\|\xi\|_{\mathcal{K}}\right)^{2}$. This the case, the assertion about $n_{q}(\rho)$ made in Lemma 7.3 follows from Lemma 7.4 if $\mathfrak{n}_{\mathfrak{q}}\left(r^{-1 / 2}\right) \leq c_{0} r^{-1}\left(r^{-1 / 2+8 \sigma}+\|\xi\|_{\mathcal{K}}\right)^{2} r^{-v / 2}$. To see that such is the case, note that $\mathfrak{n}_{\mathfrak{q}}\left(r^{-1 / 2}\right)$ is bounded by the $\rho=r^{-1 / 2}$ version of what is written on the far right hand side of (7-16). Given that the sum that appears there is bounded by $c_{0}$, the asserted bound on $\mathfrak{n}_{\mathfrak{q}}\left(r^{-1 / 2}\right)$ follows from Proposition 6.4's fifth bullet.

Consider next Lemma 7.3's assertion about $\mathfrak{n}_{\mathfrak{q}^{\prime}}(\rho)$. To this end, again write $\mathfrak{q}^{\prime}=$ $\mathfrak{q}^{\perp}-\Pi_{\xi}^{\prime} \mathfrak{q}$. The $\mathfrak{f}=\Pi_{\xi}^{\prime} \mathfrak{q}$ version of $\mathfrak{n}_{\mathfrak{f}}(\rho)$ is bounded by $c_{0}$ times the sum of $\mathfrak{n}_{\mathfrak{q}}(\rho)$ and what is written in (7-15). As noted in Step 6 in the previous subsection, the left most integral in (7-15) is bounded by $c_{0} r^{-1}\left(r^{-1 / 2+8 \sigma}+\|\xi\|_{\mathcal{K}}\right)^{2} \rho^{v}$. Meanwhile, the right most integral is bounded by $c_{0} \mathfrak{n}_{\mathfrak{q}}(\rho)$. As a consequence, the $\mathfrak{f}=\Pi_{\xi}^{\prime} \mathfrak{q}$ version of $\mathfrak{n}_{\mathfrak{f}}(\rho)$ is in no case greater than $c_{0} r^{-1}\left(r^{-1 / 2+8 \sigma}+\|\xi\|_{\mathcal{K}}\right)^{2} \rho^{v}$.

To say something about the $\mathfrak{f}=\mathfrak{q}^{\perp}$ version of $\mathfrak{n}_{\mathfrak{f}}(\rho)$, recall that $\mathfrak{q}^{\perp}$ obeys the version of (6-55) that takes $\mathfrak{f}=2 \mathfrak{q}$ and $u=-r^{1 / 2}\left(1-\Pi_{\xi}\right)\left(\mathfrak{h}^{\prime} * \mathfrak{q}\right)+r^{-1 / 2}\left(1-\Pi_{\xi}\right)\left(\mathfrak{v}^{\prime}-\mathfrak{v}_{\mathfrak{h}}^{\prime}\right)$. Thus, the bounds on $\|\mathfrak{f}\|_{\mathbb{H}}$ are again obeyed when $r \geq c_{0}$ and $\|\xi\|_{\mathcal{K}} \leq 1 / c_{0}$. An upper bound on $\mathfrak{m}_{*}$ by $c_{0} r^{-1}$ is obtained by considering how the formula for $\mathfrak{h}$ and $\mathfrak{v}$ that are give in the proof of Lemma 6.3 vary as $\xi$ varies in $\mathcal{B}$. The details of this derivation are straightforward and left to the reader. A bound by $c_{0} r^{-1} r^{-v / 2}$ on the $\mathfrak{f}=\mathfrak{q}^{\perp}$ version of $\mathfrak{n}_{\mathfrak{f}}\left(r^{-1 / 2}\right)$ is obtained from the bound on $\|\mathfrak{q}\|_{*}$ given by the last bullet of Proposition 6.4. The details of the derivation of such a bound are very similar to those given two paragraphs back that established the bound for $\mathfrak{n}_{\mathfrak{q}}\left(r^{-1 / 2}\right)$.

Proof of Lemma 7.4 There are seven steps to the proof. (The version below of Step 1 was suggested by the referee to replace an older version that required more of an explanation.)

Step 1 Note first that $\mathfrak{n}_{\mathfrak{q}}(\rho) \leq \mathfrak{n}_{\mathfrak{q}}\left(r^{-1 / 2}\right)$ when $\rho \leq r^{-1 / 2}$. Keeping this in mind, suppose that $T>1$ is given and that $T^{-1} r^{-1 / 2} \leq \rho \leq r^{-1 / 2}$. As $\rho T r^{1 / 2} \geq 1$, so it follows that $\mathfrak{n}_{\mathfrak{q}}(\rho) \leq \rho^{v} T^{v} \mathfrak{n}_{\mathfrak{q}}\left(r^{-1 / 2}\right) r^{v / 2}$. This understood, the lemma follows for suitable $\kappa$ if its claim holds with a given $T>1$ and all $\rho \leq T^{-1} r^{-1 / 2}$. A choice for $T$ is made in the next step. 
Step 2 Let $D$ again denote the disk of radius $2 r^{-1 / 2}$ that is concentric with $D_{\rho}$. Use Gaussian coordinates on $D$ so as to identify it with the radius $2 r^{-1 / 2}$ disk in $\mathbb{C}$. Use the parallel transport from the center of $D$ via the Hermitian connection on $N$ to write $N$ over $D$ as $D \times \mathbb{C}$. Let $\Lambda \subset \mathbb{C}$ denote the square lattice $\frac{1}{2} r^{-1 / 2} \mathbb{Z}^{2}$. Let $D(u)$ for $u \in \Lambda$ denote the disk with radius $2 r^{-1 / 2}$ centered on $u$.

Fix $u \in \Lambda$. Identify $q$ over $D \times D(u)$ as a section of the product $\mathbb{V}_{0} \times \mathbb{V}_{1}$ bundle as follows: Use parallel transport via the connection $A^{*}$ and the Levi-Civita connection along the radial arcs in $D \times\{u\}$ from the point $(0, u) \in D \times D(u)$ to define a product structure over $D \times\{u\}$. Then use parallel transport along the radial arcs in any given $z \in D$ version of $\{s\} \times D(u)$ to complete the construction the product structure over the whole of $D \times D(u)$.

With this product structure understood, write $\mathfrak{D}$ as the sum $\mathfrak{D}_{0}^{H}+\mathfrak{D}_{0}^{V}+\mathfrak{r}$ where $\mathfrak{D}_{0}^{V}$ and $\mathfrak{D}_{0}^{H}$ have no zero-th order term, and are such that $D_{0}^{H}$ takes derivatives solely in the direction along the $\mathbb{C}$ factor of $D \times D(u)$ while $\mathfrak{D}_{0}^{V}$ takes only derivatives along $D(u)$ factor. Meanwhile, $\mathfrak{r}$ is the zero-th order part of $\mathfrak{D}$ with respect to the given coordinates and trivialization. Note that $|\mathfrak{r}| \leq c_{0} r^{1 / 2}$.

Step 3 Let $\mathfrak{q}^{u}$ denote the $\mathbb{V}_{0} \times \mathbb{V}_{1}$ valued function on $D(u)$ whose value at any given $u^{\prime} \in D(u)$ is the average of $\mathfrak{q}$ over the part of $D \times\left\{u^{\prime}\right\}$ where the coordinate $z \in D$ obeys $\rho \leq|z| \leq 2 \rho$. As just noted, $\mathfrak{D}_{0}^{H}$ has no zero-th order part and it differentiates solely along the $D$ factor of $D \times D(u)$. As a consequence, $\mathfrak{D}_{0}^{H}$ annihilates $\mathbb{V}_{0} \oplus \mathbb{V}_{1}$ valued functions on $D \times D(u)$ that depend only on the coordinate for the $D(u)$ factor. This understood, it follows that $\mathfrak{D q}=\mathfrak{D}_{0}^{H}\left(\mathfrak{q}-\mathfrak{q}^{u}\right)+\mathfrak{D}_{0}^{V} \mathfrak{q}+\mathfrak{r q}$.

Let $\chi_{H}$ denote the function on $D$ given by $\chi(|\cdot| / 4 \rho)$ and let $\chi_{V}$ denote the function $D(u)$ given by $\chi_{V}=\chi\left(|(\cdot)-u| / 4 r^{-1 / 2}\right)$. Thus, $\chi_{H}$ is equal to 1 on $D_{\rho}$ and equal to 0 on the complement of $D_{2 \rho}$. Meanwhile, $\chi_{V}$ has compact support in $D(u)$ and is equal to 1 where the distance to $u$ is less than $r^{-1 / 2}$.

Write

$$
\begin{aligned}
\chi_{H} \chi_{V} \mathfrak{D} \mathfrak{q}=\mathfrak{D}_{0}^{H}\left(\chi_{H} \chi_{V}\left(\mathfrak{q}-\mathfrak{q}^{u}\right)\right)+\mathfrak{D}_{0}^{V}\left(\chi_{H} \chi_{V} \mathfrak{q}\right)+\mathfrak{r}\left(\chi_{H} \chi_{V} \mathfrak{q}\right) \\
+s_{H} \chi_{V}\left(\mathfrak{q}-\mathfrak{q}^{u}\right)+\chi_{H} s_{V} \mathfrak{q},
\end{aligned}
$$

where $\left|s_{H}\right| \leq c_{0}\left|d \chi_{H}\right| \leq c_{0} \rho^{-1}$ and $\left|s_{V}\right| \leq c_{0}\left|d \chi_{V}\right| \leq c_{0} r^{1 / 2}$. Let $d^{H}$ denote the exterior derivative along the $D$ factor of $D \times D(u)$ but viewed here as acting on $\mathbb{V}_{0} \times \mathbb{V}_{1}$ valued functions. Given that $\mathfrak{D}_{0}^{H}$ is elliptic on $D$, and given that the smallest Dirichlet eigenvalue of $d^{H \dagger} d^{H}$ on $D_{\rho}$ is no less than $c_{0}^{-1} \rho^{-2}$, it follows from (6-79) that

$$
\left\|\mathfrak{D}_{0}^{H}\left(\chi_{H} \chi_{V}\left(\mathfrak{q}-\mathfrak{q}^{u}\right)\right)\right\|_{2}^{2} \geq c_{0}^{-1}\left(\left\|d^{H}\left(\chi_{H} \chi_{V} \mathfrak{q}\right)\right\|_{2}^{2}+\rho^{-2}\left\|\chi_{H} \chi_{V}\left(\mathfrak{q}-\mathfrak{q}^{u}\right)\right\|_{2}^{2}\right) .
$$


By the same token, $\left\|\mathfrak{D}_{0}^{V}\left(\chi_{H} \chi_{V} \mathfrak{q}\right)\right\|_{2}^{2} \geq c_{0}^{-1}\left(\left\|d^{V}\left(\chi_{H} \chi_{V} \mathfrak{q}\right)\right\|_{2}^{2}+r\left\|\chi_{H} \chi_{V} \mathfrak{q}\right\|_{2}^{2}\right)$ where $d^{V}$ denotes the exterior derivative along the $D(u)$ factor of $D \times D(u)$, viewed as acting on $\mathbb{V}_{0} \oplus \mathbb{V}_{1}$ valued functions.

Step 4 As argued momentarily, it follows from (7-20) and its $\mathfrak{D}_{0}^{V}$ analog that

$$
\begin{aligned}
\left\|\mathfrak{D}_{0}^{H}\left(\chi_{H} \chi_{V}\left(\mathfrak{q}-\mathfrak{q}^{u}\right)\right)+\mathfrak{D}_{0}^{V}\left(\chi_{H} \chi_{V} \mathfrak{q}\right)\right\|_{2}^{2} \\
\quad \geq c_{0}^{-1}\left\|\nabla^{*}\left(\chi_{H} \chi_{V} \mathfrak{q}\right)\right\|_{2}^{2}-c_{0} \mathcal{W}-c_{0} \mathcal{R},
\end{aligned}
$$

where

$$
\mathcal{R}=r \int_{D_{2 \rho} \times D(u)}|\mathfrak{q}|^{2} \quad \text { and } \quad \mathcal{W}=\rho^{-2} \int_{D_{2 \rho}-D_{\rho}} \int_{D(u)}\left|\chi_{V}\left(\mathfrak{q}-\mathfrak{q}^{u}\right)\right|^{2} \mid
$$

Indeed, such an inequality follows from a suitable rewriting of the inner product between $\mathfrak{D}_{0}^{H}\left(\chi_{H} \chi_{V}\left(\mathfrak{q}-\mathfrak{q}^{u}\right)\right)$ and $\mathfrak{D}_{0}^{V}\left(\chi_{H} \chi_{V} \mathfrak{q}\right)$. In particular, were $\mathfrak{q}$ smooth, one could write twice this inner product as

(7-23) $\left\langle\chi_{H} \chi_{V}\left(\mathfrak{q}-\mathfrak{q}^{u}\right), \mathfrak{D}_{0}^{H \dagger} \mathfrak{D}_{0}^{V}\left(\chi_{H} \chi_{V} \mathfrak{q}\right)\right\rangle_{2}+\left\langle\chi_{H} \chi_{V} \mathfrak{q}, \mathfrak{D}_{0}^{V \dagger} D_{0}^{H}\left(\chi_{H} \chi_{V}\left(\mathfrak{q}-\mathfrak{q}^{u}\right)\right)\right\rangle_{2}$.

Here, $\langle,\rangle_{2}$ denotes the $L^{2}$ inner product. What is written in (7-23) differs from terms that are bounded by uniform multiples of $\mathcal{R}$ and $\mathcal{W}$ by

$$
\left\langle\chi_{V} \mathfrak{q}, \mathfrak{D}_{0}^{H \dagger} D_{0}^{V}\left(\chi_{H}^{2} \chi_{V} \mathfrak{q}\right)\right\rangle_{2}+\left\langle\chi_{V} \mathfrak{q}, \mathfrak{D}_{0}^{V \dagger} D_{0}^{H}\left(\chi_{H}^{2} \chi_{V}\left(\mathfrak{q}-\mathfrak{q}^{u}\right)\right)\right\rangle_{2} .
$$

Note in particular that this last expression would be bounded by uniform multiples of $\mathcal{R}$ and $\mathcal{W}$ but for the appearance of $\mathfrak{q}^{u}$ in the far right hand term. However, the part that involves $\mathfrak{q}^{u}$ can be written as $-\left\langle\chi \chi_{V}\left(\mathfrak{q}-\mathfrak{q}^{u}\right), \mathfrak{D}_{0}^{V^{\dagger}} \mathfrak{D}_{0}^{H}\left(\chi_{H}^{2} \chi_{V} \mathfrak{q}^{u}\right)\right\rangle_{2}$ up to terms that are again bounded by uniform multiples of $\mathcal{R}$ and $\mathcal{W}$. This is because the derivatives in $\mathfrak{D}_{0}^{H \dagger}$ annihilate $\mathfrak{q}^{u}$, so it can be added at an acceptable cost. With this rewriting understood, use the fact that the $\mathfrak{D}_{0}^{H} \mathfrak{q}^{u}$ vanishes to see that this term is no greater $c_{0} \mathcal{W}^{1 / 2}\left\|d^{V}\left(\chi_{H} \chi_{V} \mathfrak{q}\right)\right\|_{2}$. This then establishes (7-21) for the case when $\mathfrak{q}$ is smooth. The general case follows by taking limits.

Step 5 Hölder's inequality and the Sobolev inequality from (6-64) bound the term $\mathcal{R}$ by $c_{0} r^{1 / 2} \rho r^{-v / 2}\|\mathfrak{q}\|_{*}^{2}$; and this is no greater than $c_{0} \rho^{v}\|\mathfrak{q}\|_{*}^{2}$. The square of the $L^{2}$ norm of both the term $\chi_{H} s_{V} \mathfrak{q}$ and $\mathfrak{r}\left(\chi_{H} \chi_{V} \mathfrak{q}\right)$ that appear in (7-19) are bounded by a uniform multiple of $\mathcal{R}$, and thus also by $c_{0} \rho^{v}\|\mathfrak{q}\|_{*}^{2}$.

Meanwhile, the square of the $L^{2}$ norm of the term $s_{H} \chi_{V}\left(\mathfrak{q}-\mathfrak{q}^{u}\right)$ that appears in (7-19) is no greater than $c_{0} \mathcal{W}$. To bound the latter term, fix $u^{\prime} \in D(u)$ and note that

$$
\rho^{-2} \int_{D_{2 \rho}-D_{\rho}}\left|\chi_{V}\left(\mathfrak{q}-\mathfrak{q}^{u}\right)\right|^{2} \leq c_{0} \int_{D_{2 \rho}-D_{\rho}} \int_{D(u)}\left|d^{H}\left(\chi_{V} \mathfrak{q}\right)\right|^{2}
$$


Indeed, this follows from the fact that $\mathfrak{q}^{u}$ at any given $u^{\prime}$ is the average of $\left.q\right|_{u^{\prime}}$ on $D_{2 \rho}-D_{\rho}$.

What with (7-19) and (7-21), these last observations imply that

$$
c_{0}^{-1}\left\|\nabla^{*}\left(\chi_{H} \chi_{V} \mathfrak{q}\right)\right\|_{2}^{2} \leq \int_{D_{2 \rho}-D_{\rho}} \int_{D(u)}\left|d^{H}\left(\chi_{V} \mathfrak{q}\right)\right|^{2}+\rho^{v}\|\mathfrak{q}\|_{*}^{2}+\left\|\chi_{H} \chi_{V} \mathfrak{D} \mathfrak{q}\right\|_{2}^{2}
$$

Step 6 The analysis of $\left\|\chi_{H} \chi_{V} \mathfrak{D q}\right\|_{2}$ starts with the contribution from $r^{1 / 2} \chi_{H} \chi_{V} \mathfrak{f} * \mathfrak{q}$. Hölder's inequality with (7-20) and the Sobolev inequality from (6-64) finds that

$$
\left\|r^{1 / 2} \mathfrak{f} *\left(\chi_{H} \chi_{V} \mathfrak{q}\right)\right\|_{2}^{2} \leq c_{0} r\|\mathfrak{f}\|_{\mathbb{H}}^{2}\left\|\nabla^{*}\left(\chi_{H} \chi_{V} \mathfrak{q}\right)\right\|_{2}^{2} .
$$

Note that if $r\|\mathfrak{f}\|_{\mathbb{H}}^{2} \leq c_{0}^{-1}$, then the right hand side of (7-27) will be less than $1 / 100$ of the left hand side of (7-24). Such a bound on $r\|\mathfrak{f}\|_{\mathbb{H}}^{2}$ is assumed in what follows. Arguments much like those that lead to (7-27) find $\left\|r^{1 / 2} \mathfrak{h} *\left(\chi_{H} \chi_{V} \mathfrak{q}\right)\right\|_{2}^{2} \leq$ $c_{0} r^{-\sigma / 4}\left\|\nabla^{*}\left(\chi_{H} \chi_{V} \mathfrak{q}\right)\right\|_{2}^{2}$. Given what is assumed, $\left\|\chi_{H} \chi_{V} \mathfrak{u}\right\|_{2}^{2} \leq c_{0} \mathfrak{m}_{*} \rho^{v}$.

To continue the analysis of $\left\|\chi_{H} x_{v} \mathfrak{D q}\right\|_{2}^{2}$, remark that the square of the $L^{2}$ norm of $\chi_{H} \chi_{V} \Pi_{\xi} \mathfrak{D q}$ is bounded by $c_{0}\left(\mathcal{A}_{1}+\mathcal{A}_{2}\right)$ with $\mathcal{A}_{1}$ and $\mathcal{A}_{2}$ as defined in (6-67). It follows from [3, Lemmas 2.9 and 5.4.1] that $\mathcal{A}_{1}$ is bounded by $c_{0}\|\mathfrak{q}\|_{*}^{2} \rho^{v}$. Meanwhile, $\mathcal{A}_{2}$ is bounded by $c_{0} r^{-3 / 4} \mathfrak{n}_{\mathfrak{q}}(2 \rho)$.

The square of the $L^{2}$ norm of $\chi_{H} x_{V} r^{1 / 2} \Pi_{\xi}(\mathfrak{h} * \mathfrak{q})$ is bounded by $c_{0} \mathcal{A}_{3}$ with the latter defined as in (6-70). Arguments much like those used in Step 3 of the proof of Lemma 6.5 bound this by $c_{0} r^{-1 / 2}\|\mathfrak{q}\|_{*}^{2} \rho^{v}$.

The final term to consider is $\chi_{H} \chi_{V} r^{1 / 2} \Pi_{\xi}(\mathfrak{f} * \mathfrak{q})$. The square of the $L^{2}$ norm of this term is bounded by $c_{0} \mathcal{A}_{4}$ where $\mathcal{A}_{4}$ is given in (6-74). Meanwhile, what is written in (6-74) is bounded $c_{0} r\|\mathfrak{f}\|_{\mathbb{H}}^{2}\left(\int_{D_{2 \rho}} f^{2}\right)^{1 / 2}$ where $f(\cdot)=\int_{\pi^{-1}(\cdot)} \theta_{r}|\mathfrak{q}|^{2}$. In particular, it follows from what is said directly after (7-11) that the square of the $L^{2}$ norm of $\chi_{H} \chi_{V} r^{1 / 2} \Pi_{\xi}(\mathfrak{f} * \mathfrak{q})$ is bounded by $c_{0} r\|\mathfrak{f}\|_{\mathbb{H}}^{2}\|\mathfrak{q}\|_{*}^{2} \rho^{v}$.

Step 7 Multiply both sides of each $u \in \Lambda$ version of (7-24) by $e^{-\sqrt{r} \operatorname{dist}(\Sigma, u) / 200}$ and then sum over $\Lambda$. Given that the square of the $L^{2}$ norm of $\chi_{H}\left|d \chi_{V}\right| \mathfrak{q}$ is bounded by $\mathcal{R}$, and given that $\chi_{V}=1$ on $D_{\rho}$, the inequality that results from this sum over $\Lambda$ implies that

$$
c_{0}^{-1} \mathfrak{n}_{\mathfrak{q}}(\rho) \leq\left(1+r^{-1 / 2}\right) \mathfrak{n}_{\mathfrak{q}}(2 \rho)-\mathfrak{n}_{\mathfrak{q}}(\rho)+\left(\mathfrak{m}_{*}+\|\mathfrak{q}\|_{*}^{2}\right) \rho^{v} .
$$

Given that $r \geq c_{0}$, this last inequality finds

$$
\mathfrak{n}_{\mathfrak{q}}(\rho) \leq \lambda \mathfrak{n}_{\mathfrak{q}}(2 \rho)+c_{0}\left(\mathfrak{m}_{*}+\|\mathfrak{q}\|_{*}^{2}\right) \rho^{v},
$$


where $\lambda \leq 1-c_{0}^{-1}$ if $r \geq c_{0}$. Provided $v<c_{0}^{-1}$, this last equation can be iterated by successively $N$ times where $N$ is such that $2^{N} \rho$ is between $T^{-1} r^{-1 / 2}$ and $r^{-1 / 2}$. The resulting bound on $\mathfrak{n}_{\mathfrak{q}}(\rho)$ implies the assertion of Lemma 7.4.

\section{7.d Proof of Proposition 7.2: Part 2}

This part of the proof says more about $\Pi_{\xi}\left(\mathfrak{v}-\mathfrak{v}_{\mathfrak{h}}\right)$. To this end, write $r^{1 / 2} \Pi_{\xi}\left(\mathfrak{v}-\mathfrak{v}_{\mathfrak{h}}\right)$ as $\theta$ with $\left.\theta=\left(\left(\theta_{C}\right)_{C \in \mathcal{C}},\left(\theta_{\gamma-}\right)_{\gamma \in \Xi_{\Sigma_{-}}}\right),\left(\theta_{\gamma+}\right)_{\gamma \in \Xi_{\Sigma+}}\right) \in \mathcal{L}$. The various components of $\theta$ are described in the five steps that follow.

Step 1 This step considers $\theta_{C}$ for the cases when $C \in \mathcal{C}$ is not $\mathbb{R}$-invariant. To start, let $\mathfrak{c}$ denote the section of $\mathfrak{C}_{N, 1} \rightarrow C$ that assigns to each point the symmetric vortex. The component $\theta_{C}$ of $\theta$ is a section of $\left(\mathfrak{c}^{*} V_{1,0} \mathfrak{C}_{N, 1}\right) \otimes T^{0,1} C$ over $C-\left(\bigcup_{\mathcal{E} \in \mathcal{E}_{C}} \mathcal{E}_{2 R}\right)$. As such, it can be viewed equivalently as a section of $N \otimes T^{0,1} C$ over this same part of $C$. It follows from (6-32), (6-35) and what is said in Part 3 of Section 6.d with (2-4) and (2-5) that this section of $N$ is given by

$$
\bar{\partial} \xi_{C}+v_{C} \xi_{C}+\mu_{C} \bar{\xi}_{C}+\mathfrak{e},
$$

where $|\mathfrak{e}| \leq c_{0} r^{-1 / 2}\left(\left|\nabla \xi_{C}\right|+\left|\xi_{C}\right|+1\right)$.

Step 2 Suppose that $C=\mathbb{R} \times \gamma$ is a component of $\Sigma$ and that $q$ is its associated integer. Let $\mathfrak{c}$ denote the constant map from $C$ to the symmetric vortex in $\mathfrak{C}_{q}$. In this case, $\theta_{C}$ can be viewed as a section over $C \cap U_{C}$ of $\mathfrak{c}^{*} T_{1,0} C_{q}$. Given the first bullet of (6-32) and what is said in Step 3 of the proof of Lemma 3.8, it follows (again using (2-4), (2-5) and Part 3 of Section 6.d) that $\theta_{C}$ can be written as

$$
\theta_{k}=\bar{\partial} \mathfrak{c}_{\xi}+\left.\nabla^{1,0} \hbar\right|_{\mathfrak{c} \xi}+\mathfrak{e},
$$

where $|\mathfrak{e}| \leq c_{0} r^{-1 / 2}\left(\left|\nabla \xi_{C}\right|+\left|\xi_{C}\right|+1\right)$. Here, $\mathfrak{c}_{\xi}=\exp _{\mathfrak{c}}\left(\xi_{C}\right)$ and $\xi_{\mathrm{h}}$ is defined as in (1-9) using $\gamma$ 's version of $(\nu, \mu)$.

Step 3 Fix $\gamma \in \Xi_{\Sigma-}$ so as to consider the component $\theta_{\gamma-}$ from $\theta$. The discussion for any given $\gamma \in \Xi_{\Sigma+}$ version of $\theta_{\gamma+}$ is very much the same as what follows and so is not presented. Note that $\theta_{\gamma-}$ is a section over $(-\infty,-R] \times S^{1}$ over $\mathfrak{c}_{\gamma-}^{*} T_{1,0} \mathfrak{C}_{m_{\gamma-}}$. Consider first $\theta_{\gamma-}$ on $\left(-\infty,-R_{*}\right] \times S^{1}$. It follows from (6-38) that this section is bounded here by

$$
c_{0}\left(r^{-2} e^{\lambda w}+\left(\left|\nabla \xi_{\gamma-}\right|+\left|\xi_{\gamma-}\right|\right)\right)
$$

where $\lambda \geq c_{0}^{-1}$. 
Step 4 This step with Step 5 describe $\theta_{\gamma-}$ on $\left[-R_{*},-R\right] \times S^{1}$. To start the story, reintroduce the subsets $X_{2}$ and $X_{3}$ of $U_{\gamma-}$ as defined in Part 4 of the proof of Lemma 6.3. Part 5 of the proof of Lemma 6.3 describes $\mathfrak{v}-\mathfrak{v}_{\mathfrak{h}}$ on $X_{2}$ as a map from $\mathbb{R} \times S^{1} \times \mathbb{C}$

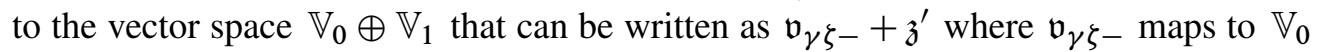
and is given by (6-39). Meanwhile, it follows from (6-40)-(6-42) that

$$
\left|\mathfrak{z}^{\prime}\right| \leq c_{0} r_{\gamma}^{-1 / 2}\left(1+r \rho_{*}^{2}\right)\left(1+\left|\nabla \xi_{\gamma-}\right|+\left|\xi_{\gamma-}\right|\right) \sum_{\left.z^{\prime} \in Z_{\gamma-}\right|_{(w, t)}} e^{-\sqrt{r}\left|z-z^{\prime}\right| / 2} .
$$

Given what is said about $\mathfrak{v}-\mathfrak{v}_{\mathfrak{h}}$ on $X_{3}$ in Part 6 of the proof of Lemma 6.3 and given (2-4) and (2-5), it follows that $\mathfrak{v}-\mathfrak{v}_{\mathfrak{h}}$ can be written here as $r_{\gamma}^{1 / 2} \mathfrak{v}_{\gamma \zeta-}+\mathfrak{z}^{\prime}$ where $\mathfrak{v}_{\gamma \xi-}$ is also given by (6-39), and where (7-33) describes $\mathfrak{z}^{\prime}$

Step 5 Reintroduce the notation from Section 5.c. View $\mathfrak{c}_{\gamma-}^{*} T_{1,0} \mathfrak{C}_{m_{\gamma-}}$ over $I_{k} \times S^{1}$ as isomorphic to $\mathfrak{c}_{k}^{*} T_{1,0} C_{m_{k}} \oplus\left(\bigoplus_{\mathcal{E} \in \mathcal{E}_{\gamma-}-E_{k}} \pi_{*}\left(\left.N\right|_{\mathcal{E}}\right)\right)$ where $\pi$ is the map in (5-18) and $N$ is the normal bundle to $\mathcal{E}$. This isomorphism writes $\theta_{\gamma-}$ as $\left(\theta_{k}, \pi_{*}\left(\theta_{\Sigma k}\right)\right)$. It follows using (2-4), (2-5), (6-9), (6-39) and what was said in the preceding step that $\theta_{k}$ can be written

$$
\theta_{k}=\bar{\partial} \mathfrak{c}_{k, \xi}+\left.\nabla^{1,0} \hat{h}\right|_{\mathfrak{c}_{k, \xi}}+\mathfrak{e}
$$

and where $|\mathfrak{e}| \leq r^{-1 / 2+6 \sigma}\left(1+\left|\nabla \xi_{\gamma-}\right|+\left|\xi_{\gamma-}\right|\right)$. Here, the notation uses $\mathfrak{c}_{k, \xi}: I_{k} \times S^{1} \rightarrow$ $C$ to denote $\exp _{\mathfrak{c}_{k}}\left(\phi_{k}^{\dagger}\left(\zeta_{*}+\xi_{\gamma-}\right)\right)$ with $\zeta_{*}$ as described in Step 5 of Section 3.d. Meanwhile, $\bar{\partial}$ denotes $(1 / 2)(\partial / \partial w+i(\partial / \partial t))$. Finally, $f$ denotes the function on $\mathfrak{C}_{m_{k}}$ that is given by the version of (1-9) that is defined by $\gamma$ 's pair $(\nu, \mu)$. The argument from Step 3 of the proof of Lemma 3.8 can be used to prove this assertion about $\theta_{k}$.

Meanwhile, (2-4), (2-5), (5-12) and (6-9) imply that $\theta_{\Sigma k}$ here is also given on any given end: $\mathcal{E} \in \mathcal{E}_{\gamma_{-}}-\mathcal{E}_{k}$ by the relevant version of (7-30).

\section{7.e Proof of Proposition 7.2: Part 3}

This part of the proof describes the terms $\mathcal{T}_{0}, \mathcal{T}_{1}$ and $\mathcal{T}_{2}$ that appear in (7-2). The discussion has three parts.

Part 1 This part discusses $\mathcal{T}_{0}=\mathcal{T}(0)$. As an element in the Banach space $\mathcal{L}$, it can be written as $\mathcal{T}_{0}=\left(\left(\mathcal{T}_{0 C}\right)_{C \in \mathcal{C}},\left(\mathcal{T}_{0 \gamma-}\right)_{\gamma \in \Xi_{\Sigma-}},\left(\mathcal{T}_{0 \gamma+}\right)_{\gamma \in \Xi_{\Sigma+}}\right)$. It follows from (7-4)(7-6), (7-12) and by (7-30) that $\left\|\mathcal{T}_{0 C}\right\|_{\mathcal{L}} \leq c_{0} r^{-1 / 2+8 \sigma}$ if $C \in \mathcal{C}$ is not $\mathbb{R}$-invariant. Suppose next that $C=\mathbb{R} \times \gamma$ with associated integer $q$. As the constant map from $\mathfrak{c}$ to the symmetric vortex obeys the equation $\bar{\partial} \mathfrak{c}+\mathfrak{c}^{*} \nabla^{1,0} \hat{h}=0$, it follows from (7-31) with (7-5), (7-6) and (7-12) that $\left\|\mathcal{T}_{0 C}\right\|_{\mathcal{L}}$ is again bounded by $c_{0} r^{-1 / 2+8 \sigma}$.

Consider next a given $\gamma \in \Xi_{\Sigma-}$ version of $\mathcal{T}_{0 \gamma-}$. Introduce the notation from Section 5.c to view $\mathfrak{c}_{\gamma-}^{*} T_{1,0} \mathfrak{C}_{m_{\gamma-}}$ over $I_{k} \times S^{1}$ as $c_{k}^{*} T_{1,0} \mathfrak{C}_{m_{k}} \oplus\left(\bigoplus_{\mathcal{E} \in \mathcal{E}_{\gamma-}-E_{k}} \pi_{*}\left(\left.N\right|_{\mathcal{E}}\right)\right)$ 
where $\pi$ is the map in (5-18). Viewed in this way, $\mathcal{T}_{0 \gamma-}=\left(\mathcal{T}_{0 k}, \pi_{*}\left(\mathcal{T}_{0 \Sigma k}\right)\right)$. It follows from (7-4)-(7-6) and (7-12) with (7-30), (7-31) and the formula in Step 4 in Section 5.b that $\left\|\mathcal{T}_{0 \Sigma k}\right\|_{\mathcal{L}}$ is again bounded by $c_{0} r^{-1 / 2+8 \sigma}$. Meanwhile, it follows from (7-4)-(7-6) and (7-12) with (7-32)-(7-34) that $\mathcal{T}_{0 k}$ has the same form as the $\xi=0$ version of (7-34). What is written as $\mathfrak{e}$ in this new, $\mathcal{T}_{0 k}$ version of (7-34) obeys $\|\mathfrak{e}\|_{\mathcal{L}} \leq c_{0} r^{-1 / 2+8 \sigma}$. At the same time, (4-2), Constraint 2 in Section 4.b and (4-9) imply that

$$
\left|\bar{\partial} \mathfrak{c}_{k}+\nabla^{1,0} \mathfrak{h}\right|_{\mathfrak{c}_{k}} \mid \leq c_{0} r^{-1 / 2+5 \sigma} e^{2 \lambda s},
$$

where $\lambda>c_{0}^{-1}$. As a consequence, $\left\|\mathcal{T}_{0 k}\right\|_{\mathcal{L}} \leq c_{0} r^{-1 / 2+8 \sigma}$ also. Thus, $\left\|\mathcal{T}_{0 \gamma-}\right\|_{\mathcal{L}} \leq$ $c_{0} r^{-1 / 2+8 \sigma}$.

An analogous argument proves that $\mathcal{T}_{0 \gamma+} \|_{\mathcal{L}} \leq c_{0} r^{-1 / 2+8 \sigma}$ for each $\gamma \in \Xi_{\Sigma+}$. As a consequence, $\left\|\mathcal{T}_{0}\right\|_{\mathcal{L}} \leq c_{0} r^{-1 / 2+8 \sigma}$. This gives the first bullet in Proposition 7.2.

Part 2 What is written as $\mathcal{T}_{1}$ in (7-2) and Proposition 7.2 is defined as follows: Let $\xi^{\prime}$ denote a section of $\mathcal{K}$. Then $\mathcal{T}_{1}\left(\xi^{\prime}\right)$ is the derivative $\ln$ the direction of $\xi^{\prime}$ at $\xi=0$ of the map that sends $\xi \in \mathcal{B}$ to $r^{1 / 2} \Pi_{\xi}\left(\mathfrak{v}-\mathfrak{v}_{\mathfrak{h}}\right)$. The map that sends $\xi$ to $r^{1 / 2} \Pi_{\xi}\left(\mathfrak{v}-\mathfrak{v}_{\mathfrak{h}}\right)$ is denoted in what follows by $\mathcal{P}$. Note that $\mathcal{P}$ is a smooth map from $\mathcal{B}$ to $\mathcal{L}$.

The second bullet of Proposition 7.2 asserts that $\mathcal{T}_{1}$ is an isomorphism when restricted to the kernel of the map $q$, as yet to be define. It also asserts an upper bound for $\mathcal{T}_{1}^{-1}$. These two assertions about $\mathcal{T}_{1}$ are proved in the next subsection. What follows provides a description of $\mathcal{T}_{1}$ for use in these proofs.

To start, write $\mathcal{T}_{1}(\xi)=\left(\left(\mathcal{T}_{1 C}\right)_{C \in \mathcal{C}},\left(\mathcal{T}_{1 \gamma-}\right)_{\gamma \in \Xi_{\Sigma-}},\left(\mathcal{T}_{1 \gamma+}\right)_{\gamma \in \Xi_{\Sigma+}}\right)$ and consider first $\mathcal{T}_{1 C}$ when $C \in \mathcal{C}$ is not $\mathbb{R}$-invariant. It follows from (7-30) with (6-32) and (6-35) that

$$
\mathcal{T}_{1 C}=\bar{\partial} \xi_{C}+v_{C} \xi_{C}+\mu_{C} \bar{\xi}_{C}+\mathfrak{e},
$$

where $\|\mathfrak{e}\|_{\mathcal{L}^{2}} \leq c_{0} r^{-1 / 2+8 \sigma}\|\xi\|_{\mathcal{K}_{1}^{2}}$ and $\|\mathfrak{e}\|_{L} \leq c_{0} r^{-1 / 2+8 \sigma}\|\xi\|_{\mathcal{K}}$.

Suppose next that $C=\mathbb{R} \times \gamma$ is a component of $\Sigma$. Let $q$ denote its associated integer. Let $\mathfrak{c}$ denote the constant map to the symmetric vortex in $\mathfrak{C}_{q}$. Identify $\left.T_{1,0} \mathfrak{C}_{q}\right|_{c}=\mathbb{C}^{q}$ using the coordinates in (1-5). This identification makes $\mathcal{T}_{1 C}$ a map from the relevant part of $C$ to $\mathbb{C}^{q}$. If $q=1$, then (7-31) with (6-32) and (6-35) can be used to prove that this map is given by (7-36). If $q>1$, then (7-31) with (6-32) and (6-35) can be used to prove that the $q$-th component of $\mathcal{T}_{1 C}$ is

$$
\bar{\partial} \xi_{C q}+\frac{1}{2} q \mathrm{R} \xi_{C q}+\mathfrak{e},
$$

where $\xi_{C q}$ is the $q$-th component of $\xi_{C}$ and where $\mathrm{R}$ is the rotation number assigned to $\gamma$. Meanwhile, $\mathfrak{e}$ again obeys $\|\mathfrak{e}\|_{\mathcal{L}^{2}} \leq c_{0} r^{-1 / 2+8 \sigma}\|\xi\|_{\mathcal{K}_{1}^{2}}$ and $\|\mathfrak{e}\|_{\mathcal{L}} \leq$ $c_{0} r^{-1 / 2+8 \sigma}\|\xi\|_{\mathcal{K}}$. 
Consider next $\mathcal{T}_{1 \gamma-}$. With $\mathfrak{c}_{\gamma-}^{*} T_{1,0} \mathfrak{C}_{m_{\gamma-}}$ over $I_{k} \times S^{1}$ again viewed as the bundle $\mathfrak{c}_{k}^{*} T_{1,0} \mathfrak{C}_{m_{k}} \oplus\left(\bigoplus_{\mathcal{E} \epsilon_{\gamma-}-\mathcal{E}_{k}} \pi_{*}\left(\left.N\right|_{\mathcal{E}}\right)\right.$, write $\mathcal{T}_{1 \gamma-}=\left(\mathcal{T}_{1 k}, \pi_{*}\left(\mathcal{T}_{1 \Sigma k}\right)\right)$. In this regard, $\mathcal{T}_{1 \Sigma k}$ is also given by the relevant version of (7-36); the arguments alluded to in the previous paragraph can be used if augmented with (2-4), (2-5), (2-11), (2-12) and (4-9). Meanwhile, $\mathcal{T}_{1 k}$ can be written as

$$
\mathcal{T}_{1 k}=\bar{\partial}_{\mathfrak{c}_{k}} \xi_{k}+\left.\left(\nabla_{\xi_{R}} \nabla^{1,0} h\right)\right|_{\mathfrak{c}_{k}}+\mathfrak{e}
$$

where the notation is as follows: First, $\bar{\partial}_{\mathfrak{c}_{k}}$ denotes the $d$-bar operator on the space of sections of $\mathfrak{c}_{k}^{*} T_{1,0} \mathfrak{C}_{m_{k}}$ that is defined using the pullback via $\mathfrak{c}_{k}$ of the Levi-Civita connection. Second, $\xi_{R}$ denotes the section of $T \mathfrak{C}_{m_{k}}$ that is defined by the section $\xi$ of $T_{1,0} \mathfrak{C}_{m_{k}}$. Third, the term $\mathfrak{e}$ obeys $\|\mathfrak{e}\|_{\mathcal{L}^{2}} \leq c_{0} r^{-1 / 2+8 \sigma}\|\xi\|_{\mathcal{K}_{1}^{2}}$ and $\|\mathfrak{e}\|_{\mathcal{L}} \leq$ $c_{0} r^{-1 / 2+8 \sigma}\|\xi\|_{\mathcal{K}}$. The identification of $\mathcal{T}_{1 k}$ in (7-38) follows from (7-32)-(7-34).

There is an analogous description of $\mathcal{T}_{1 \gamma+}$.

Part 3 This part considers $\mathcal{T}_{2}$ in Proposition 7.2. This term is equal to

$$
r^{1 / 2} \Pi_{(\cdot)}\left(\mathfrak{D} \mathfrak{q}+r^{1 / 2} \mathfrak{q} * \mathfrak{q}+2 r^{1 / 2} \mathfrak{h} * \mathfrak{q}\right)-\mathcal{T}_{0}+\mathcal{P}-\mathcal{T}_{1}
$$

To say more about (7-39), write $\mathcal{T}_{0}=\left.\mathcal{P}\right|_{0}+\mathcal{R} 0$, and introduce $\mathcal{P}_{2}=-\mathcal{P}-\left.\mathcal{P}\right|_{0}-\mathcal{T}_{1}$. Arguments much like those that imply (7-30)-(7-34) can be employed to prove that

$$
\left\|\mathcal{P}_{2}(\xi)\right\|_{\mathcal{L}} \leq c_{0}\|\xi\|_{\mathcal{K}}^{2} \text { and }\left\|\mathcal{P}_{2}(\xi)-\mathcal{P}_{2}\left(\xi^{\prime}\right)\right\|_{\mathcal{K}} \leq c_{0}\left(\|\xi\|_{\mathcal{K}}+\left\|\xi^{\prime}\right\|_{\mathcal{K}}\right)\left\|\xi-\xi^{\prime}\right\|_{\mathcal{K}}
$$

Now let $\mathcal{R}=r^{1 / 2} \Pi_{(\cdot)}\left(\mathfrak{D} \mathfrak{q}+r^{1 / 2} \mathfrak{q} * \mathfrak{q}+2 r^{1 / 2} \mathfrak{h} * \mathfrak{q}\right)-\mathcal{R} 0$. By construction $\mathcal{R}(0)=0$. Let $\xi \in \mathcal{B}$ and let $\xi^{\prime}$ denote a unit length element in $\mathcal{K}$. The derivative of $\mathcal{R}$ in the direction $\xi^{\prime}$ has two contributions, these precise analogs of the two contributions to the differential of $\mathcal{T}$ that are described in Step 5 of Section 7.b. The first contribution is

$$
-r^{1 / 2} \Pi_{\xi} D \Pi_{\xi^{\prime}} q+r^{1 / 2} \Pi_{\xi}\left(\mathfrak{D} \mathfrak{q}^{\perp}+2 r^{1 / 2} \mathfrak{q} * \mathfrak{q}^{\prime}+2 r^{1 / 2} \mathfrak{h} * \mathfrak{q}^{\prime}+\mathfrak{h}^{\prime} * \mathfrak{q}\right),
$$

where the primes denote directional derivatives, and where $\mathfrak{q}^{\prime}$ is written as $\mathfrak{q}^{\perp}-\Pi_{\xi}^{\prime} \mathfrak{q}$ with $\mathfrak{q}^{\perp} \in \mathbb{H}^{\perp}$. Meanwhile, the $\mathcal{L}$ norm of the second contribution is bounded by $c_{0}$ times the $\mathcal{L}$ norm of $r^{1 / 2} \Pi_{(\cdot)}\left(\mathfrak{D q}+r^{1 / 2} \mathfrak{q} * \mathfrak{q}+2 r^{1 / 2} \mathfrak{h} * \mathfrak{q}\right)$.

Consider first the $\mathcal{L}$ norm of $r^{1 / 2} \Pi_{(\cdot)}\left(\mathfrak{D q}+r^{1 / 2} \mathfrak{q} * \mathfrak{q}+2 r^{1 / 2} \mathfrak{h} * \mathfrak{q}\right)$. It follows from (7-4)-(7-6) that its $\mathcal{L}$ norm is bounded by $c_{0}\left(r^{-1 / 2+8 \sigma}+\|\xi\|_{\mathcal{K}}\right)^{2}$. Meanwhile, Step 6 in Section 7.c argues that the $\mathcal{L}$ norm of $r^{1 / 2} \Pi_{\xi} \mathfrak{D} \Pi_{\xi}^{\prime} \mathfrak{q}$ is bounded by $c_{0}\left(r^{-1 / 2+8 \sigma}+\|\xi\|_{\mathcal{K}}\right)$. Step 7 in Section 7.c explains why the $\mathcal{L}$ norm of what remains in (7-41) is never greater than $\left(r^{-1 / 4+8 \sigma}+\|\xi\|_{\mathcal{K}}\right)$. 
The bounds just derived for the differential of $\mathcal{R}$ imply that

- $\|\mathcal{R}(\xi)\|_{\mathcal{L}} \leq c_{0}\left(r^{-1 / 4+8 \sigma}+\|\xi\|_{\mathcal{K}}\right)\|\xi\|_{\mathcal{K}}$.

- $\left\|\mathcal{R}(\xi)-\mathcal{R}\left(\xi^{\prime}\right)\right\|_{\mathcal{K}} \leq c_{0}\left(r^{-1 / 4+8 \sigma}+\|\xi\|_{\mathcal{K}}+\left\|\xi^{\prime}\right\|_{\mathcal{K}}\right)\left\|\xi-\xi^{\prime}\right\|_{\mathcal{K}}$.

Taken together, (7-40) and (7-41) imply the last two bullets in Proposition 7.2.

\section{7.f Proof of Proposition 7.2: Part 4}

This subsection defines the vector space $V_{0}$ and the map $\mathfrak{q}: \mathcal{K} \rightarrow V_{0}$ that are mentioned in Proposition 7.1 and Proposition 7.2. To start, $V_{0}$ can be written as the direct sum of spaces, these indexed by the elements in $\mathcal{C}$, the elements in $\Xi_{\gamma-}$ and the elements in $\Xi_{\gamma+}$. In this regard, any given $C \in \mathcal{C}$ contributes as follows to $V_{0}$ : If $C=\mathbb{R} \times \gamma$, then $C$ contributes nothing. If $C$ is not $\mathbb{R}$-invariant, then $C$ contributes the summand $\operatorname{kernel}\left(\mathcal{D}_{C}\right)$, where $\mathcal{D}_{C}$ is the operator that appears in (4-5). Here, and below, $\operatorname{kernel}(\cdot)$ refers to the $L^{2}$ kernel.

Consider next the summand in $V_{0}$ that is labeled by any given $(\gamma, m) \in \boldsymbol{\Xi}_{-}$. If $m=1$, then this pair contributes nothing to $\Xi_{-}$. To set the stage for the description of $(\gamma, m)$ 's summand when $m>1$, digress momentarily and suppose that $\mathcal{E} \subset \mathcal{E}_{\gamma-}$. In the case when $\mathcal{E}$ is not the $s \ll-1$ part of $\mathbb{R} \times \gamma$, assign to $\mathcal{E}$ the configuration $\mathfrak{Z}_{\mathcal{E}_{0}} \in \operatorname{Sym}^{q_{\mathcal{E}}}(\mathbb{C})$ that is obtained by replacing $z_{\mathcal{E}}$ in (5-16) with $\varsigma_{q_{\mathcal{E}}} e^{-2 \lambda_{q \mathcal{E}} w}$. Here, $\varsigma_{q_{\mathcal{E}}}$ is the eigenvector that appears in $\mathcal{E}$ 's version of (4-2) and $\lambda_{q_{\mathcal{E}}}$ denotes the corresponding eigenvalue. If $\mathcal{E}$ is the $s \ll-1$ part of $\mathbb{R} \times \gamma$, assign to $\mathcal{E}$ the point in $\operatorname{Sym}^{q_{\mathcal{E}}}(\mathbb{C})$ with all entries 0 .

With these preliminaries out of the way, introduce the notation from Section 5.c. As in Section 5.c, let $N=N_{\gamma-}$ denote the number of distinct elements $\left\{\lambda_{q_{\mathcal{E}}}: \mathcal{E} \in \mathcal{E}_{\gamma-}\right\}$. The $(\gamma, m)$ contribution to $V_{0}$ is itself a direct sum, with the summands labeled by the integers $k \in\{1, \ldots, N\}$. To describe the $k$-th summand, introduce the partition subset $\mathcal{E}_{k} \subset \mathcal{E}_{\Sigma \gamma-}$ as defined in Section 5.c. Define $\left.\mathfrak{Z}_{k 0}\right|_{(w, t)} \in \operatorname{Sym}^{m_{k}}(\mathbb{C})$ by replacing each $\mathcal{E} \in \mathcal{E}_{k}$ version of $\mathfrak{Z}_{\mathcal{E}}$ in (5-17) by $\mathfrak{Z}_{\mathcal{E}_{0}}$ and by replacing each $\mathcal{E} \in \bigcup_{j>k} \mathcal{E}_{j}$ version of $\mathfrak{Z}_{\mathcal{E}}$ by the point in $\operatorname{Sym}^{q_{\mathcal{E}}}(\mathbb{C})$ with all entries equal to 0 . When $k<N$, the configuration $\left.\mathfrak{Z}_{k 0}\right|_{(w, t)}$ consists of $m_{k}-m_{k+1}$ nonzero points and $m_{k+1}$ points at the origin. Meanwhile, $\left.\mathfrak{Z}_{N 0}\right|_{(w, t)}$ has either all points distinct and nonzero or all points at the origin.

Use the configuration $r_{\gamma}^{1 / 2} \mathfrak{Z}_{k 0}$ to define a map $\mathfrak{c}_{k 0}: \mathbb{R} \times S^{1} \rightarrow \mathfrak{C}_{m_{k}}$. Note that the domain of this map is the whole of $\mathbb{R} \times S^{1}$ to $\mathfrak{C}_{m_{k}}$. There is, as usual, an analogous map $\mathfrak{c}_{k 0}: \mathbb{R} \rightarrow \mathfrak{C}_{m_{k}}$ for any given $k \in\left\{1, \ldots, N_{\gamma+}\right\}$ if $(\gamma, m) \in \Xi_{\Sigma+}$. The next lemma refers to the version of $\mathfrak{c}_{k 0}$ that is defined either with $(\gamma, m) \in \Xi_{\Sigma-}$ and $k \in N_{\gamma-}$ or with $(\gamma, m) \in \Xi_{\Sigma+}$ and $k \in N_{\gamma+}$. The lemma also refers to the function $\hbar$ on $\mathfrak{C}_{m_{k}}$ that is defined in (1-9) by taking $\mu=0$ and $\nu$ to be half of $\gamma$ 's rotation number. 
Lemma 7.5 If all but Constraint 4 in Section $4 . b$ are satisfied, then

$$
\zeta \rightarrow \bar{\partial}_{\mathfrak{c}_{k 0}} \zeta+\left.\left(\nabla_{\zeta_{\mathbb{R}}} \nabla^{1,0} h\right)\right|_{\mathfrak{c}_{k 0}}
$$

is Fredholm from $L_{1}^{2}\left(\mathbb{R} \times S^{1} ; \mathfrak{c}_{k 0}^{*} T_{1,0} \mathfrak{C}_{m_{k}}\right)$ to $L^{2}\left(\mathbb{R} \times S^{1} ; \mathfrak{c}_{k 0}^{*} T_{1,0} \mathfrak{C}_{m_{k}}\right)$ with trivial cokernel.

This lemma is proved in Section 7.i. Assume it for the time being.

As any given version of Lemma 7.5's operator is Fredholm, it has finite dimensional $L^{2}$ kernel. The $(\gamma, m) \in \Xi_{\Sigma-}$ and $k \in\left\{1, \ldots, N_{\gamma-}\right\}$ version of this kernel is denoted by $V_{\gamma-, k}$; the $(\gamma, m) \in \Xi_{\Sigma+}$ and $k \in\left\{1, \ldots, N_{\gamma+}\right\}$ version is denoted by $V_{\gamma+, k}$. Granted this notation, $\mathbb{V}_{0}$ can be written as

$$
\begin{aligned}
V_{0}=\left(\bigoplus_{C \in \mathcal{C}} \operatorname{kernel}\left(\mathcal{D}_{C}\right)\right) \oplus\left(\bigoplus_{\gamma \in \Xi_{\Sigma-}}\left(\bigoplus_{1 \leq k \leq N_{\gamma-}} V_{\gamma-, k}\right)\right) \\
\oplus\left(\bigoplus_{\gamma \in \Xi_{\Sigma+}}\left(\bigoplus_{1 \leq k \leq N_{\gamma+}} V_{\gamma+, k}\right)\right) .
\end{aligned}
$$

The norm on $V_{0}$ is the direct sum of the $L^{2}$ inner products for each of the various summands.

The next order of business is to define the map $q: \mathcal{K} \rightarrow V_{0}$. This requires a digression in order to describe a certain useful decomposition of any given element $\xi \in \mathcal{K}$ as a sum

$$
\xi=\sum_{C \in \mathcal{C}} \xi^{C}+\sum_{\gamma \in \Xi_{\Sigma-}} \xi^{\gamma-}+\sum_{\gamma \in \Xi_{\Sigma+}} \xi^{\gamma+}
$$

that are determined by their namesake components of

$$
\xi=\left(\left(\xi_{C}\right)_{C \in \mathcal{C}},\left(\xi_{\gamma-}\right)_{\gamma \in \Xi_{\Sigma-}},\left(\xi_{\gamma+}\right)_{\gamma \in \Sigma_{+}}\right) .
$$

Consider first $\xi^{C}$. As $\xi^{C} \in \mathcal{K}$, it has components

$$
\xi^{C}=\left(\left(\xi_{C^{\prime}}^{C}\right)_{C^{\prime} \in C},\left(\xi_{\gamma-}^{C}\right)_{\gamma \in \Xi_{\Sigma-}},\left(\xi_{\gamma+}^{C}\right)_{\gamma \in \Xi_{\Sigma+}}\right) .
$$

These are as follows: First, $\xi_{C^{\prime}}^{C}=0$ unless $C=C^{\prime}$. In this case, $\xi_{C}^{C}=\left(1-\sum_{\mathcal{E} \in \mathcal{E}_{C}} \chi_{\mathcal{E}}\right) \xi_{C}$ where the sum is indexed by the ends in $C$. By way of a reminder, the cut-off function $\chi_{\mathcal{E}}$ is zero on the complement of $\mathcal{E}$ and defined on $\mathcal{E}$ by $\chi\left(\left(R_{\mathcal{E}}+3 R-|w|\right) / R\right)$. Meanwhile, any given $\xi_{\gamma \pm}^{C}$ is determined by $\left(\xi_{C^{\prime}}^{C}\right)_{C^{\prime} \in C}$ through the homomorphism

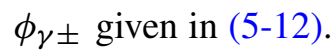

As an element of $\mathcal{K}$, the term $\xi^{\gamma-}$ from (7-44) has only nonzero components $\xi_{\gamma-}^{\gamma-}$ and $\xi_{C}^{\gamma-}$ for those $C$ with $C \cap U_{\gamma-} \neq \varnothing$. In particular, $\xi_{\gamma^{\prime}-}^{\gamma-}=0$ if $\gamma^{\prime} \neq \gamma$, and also $\xi_{\gamma^{\prime}+}^{\gamma-}=0$ for all $\gamma^{\prime} \in \Xi_{\Sigma+}$. The component $\xi_{\gamma-}^{\gamma-}$ is set equal to $\xi_{\gamma-}-\xi_{\gamma-}^{C}$. The component $\xi_{C}^{\gamma-}$ is determined from $\xi_{\gamma-}^{\gamma-}$ by the identifications in (5-12). Note in particular that $\xi_{\gamma-}^{\gamma-}$ has support where $w<-3 R$. Any given $\xi^{\gamma+}$ has a similar description. 
Now fix $(\gamma, m) \in \Xi_{\Sigma-}$ so as to describe a further decomposition of $\xi^{\gamma-}$. To this end, reintroduce again the notation from Section 5.c. With this notation understood, fix $k$ and write $\xi^{\gamma-}$ on $I_{k} \times S^{1}$ as $\left(\xi_{k}^{\gamma-}, \pi_{*}\left(\xi_{\Sigma k}^{\gamma-}\right)\right)$. Note that the term $\xi_{\Sigma k}^{\gamma-}$ is given by $\xi_{\Sigma k}^{\gamma-}=\sum_{\mathcal{E} \in \mathcal{E}_{\gamma-}-\mathcal{E}_{k} \text { and } \mathcal{E} \subset C} \chi_{\mathcal{E}} \xi_{C}$. This means, in particular, that $\xi_{\Sigma k}^{\gamma \underline{\Sigma}} \neq 0$ only at values of $w$ with distance $2 R$ or less from the left endpoint of $I_{k}$; this where $I_{k-1} \cap I_{k} \neq 0$ and where the ends in $\mathcal{E}_{k-1}$ have $\chi_{\mathcal{E}} \neq 0$. With the preceding understood, agree now to decompose $\xi^{\gamma-}$ as

$$
\xi^{\gamma-}=\xi^{\gamma-1}+\xi^{\gamma-2}+\cdots+\xi^{\gamma-N} .
$$

Here $\xi^{\gamma-k}$ has compact support on $I_{k} \times S^{1}$ and is given by

$$
\xi^{\gamma-k}=\left(\chi_{k} \xi_{k}^{\gamma-}, 0\right)
$$

with $\chi_{k}: I_{k} \rightarrow[0,1]$ defined as follows: Let $R_{k}$ denote the value of $R_{\mathcal{E}}$ for the ends $\mathcal{E} \in \mathcal{E}_{k}$. Then $\chi_{k}=\chi\left(\left(R_{k}+2 R-|w|\right) / R\right)\left(1-\chi\left(\left(R_{k-1}+2 R-|w|\right) / R\right)\right.$. There is, as usual, the analogous decomposition of any given $(\gamma, m) \in \Xi_{\Sigma+}$ version of $\xi^{\gamma+}$.

With these preliminaries set, what follows describes the map $q: K \rightarrow \mathbb{V}_{0}$. To start, fix $C \in \mathcal{C}$. Then the image of $q(\xi)$ in the summand $\operatorname{kernel}\left(\mathcal{D}_{C}\right) \subset \mathbb{V}_{0}$ is the $L^{2}$ orthogonal projection of $\xi_{C}^{C}$ onto the kernel of $\mathcal{D}_{C}$. In order to define the image of $q(\xi)$ in any given $k \in\left\{1, \ldots, N_{\gamma-}\right\}$ and $(\gamma, m) \in \Xi_{\Sigma-}$ version of the summand $V_{\gamma-, k}$, it is necessary to first note that the maps $\mathfrak{c}_{k 0}$ and $\mathfrak{c}_{k}$ are very close where both are defined, on $I_{k} \times S^{1} \subset \mathbb{R} \times S^{1}$. Indeed, it follows from Constraint 2 in Section 4.b that the distance in $\mathfrak{C}_{m_{k}}$ between $\left.\mathfrak{c}_{k}\right|_{(w, t)}$ and $\left.\mathfrak{c}_{k 0}\right|_{(w, t)}$ is bounded by $c_{0} r^{-\sigma / 4}$ at each $(w, t) \in I_{k} \times S^{1}$. This implies that the map $\exp _{\mathfrak{c}_{k 0}}$ from Part 8 of Section 2.a can be used to identify the $\mathfrak{c}_{k 0}$ and $\mathfrak{c}_{k}$ pullbacks over $I_{k} \times S^{1}$ of $T_{1,0} \mathfrak{C}_{m_{k}}$ when $r \geq c_{0}$. This identification is used to view $\chi_{k} \xi_{k}^{\gamma-}$ as a section of $\mathfrak{c}_{k 0}^{*} T_{1,0} \mathfrak{C}_{m_{k}}$. Granted this identification, the image of $q(\xi)$ in the summand $V_{\gamma-, k}$ is defined to be the $L^{2}$ orthogonal projection of $\chi_{k} \xi_{k}^{\gamma-}$ onto $V_{\gamma-, k}$. There is an analogous definition for the image of $q(\xi)$ in any given $k \in\left\{1, \ldots, N_{\gamma+}\right\}$ and $(\gamma, m) \in \Xi_{\Sigma+}$ version of $V_{\gamma+, k}$.

The upper bound asserted by Proposition 7.2 for $|q(\xi)|$ follows from the definition of any given component of $\mathfrak{q}$ as an $L^{2}$ projection.

The final task for this subsection is to explain why $q: \mathcal{K} \rightarrow V_{0}$ is surjective when $R$ and $r$ are large. To this end, fix $C \in \mathcal{C}$. Let $o \in \operatorname{kernel}\left(\mathcal{D}_{C}\right)$ and use $o$ to define an element $\xi^{o}=\left(\left(\xi_{C^{\prime}}^{o}\right)_{C^{\prime} \in C}\left(\xi_{\gamma-}^{o}\right)_{\gamma \in \Xi_{\Sigma_{-}}},\left(\xi_{\gamma+}^{o}\right)_{\gamma \in \Xi_{\Sigma+}}\right) \in \mathcal{K}$ as follows: Set $\xi_{C^{\prime}}^{o}=0$ unless $C=C^{\prime}$ and set $\xi_{C}^{o}=\left(1-\sum_{\mathcal{E} \in \mathcal{E}_{C}} \chi_{\mathcal{E}}^{\prime}\right) o$ where $\chi_{\mathcal{E}}^{\prime}$ is the function on $C$ with compact support in $\mathcal{E}$ and given on $\mathcal{E}$ by the restriction of $\chi\left(\left(R_{\mathcal{E}}+3.5 R-|w|\right) / R\right)$. Define $\xi_{\gamma-}^{o}$ for $\gamma \in \Xi_{-}$to equal zero unless $C$ intersects $U_{\gamma-}$. In the latter case, use (6-18) to define $\xi_{\gamma_{-}}^{o}$. Make the analogous definitions of $\left(\xi_{\gamma+}^{o}\right)_{\gamma \in \Xi_{\Sigma+}}$. Let $o_{V} \in V_{0}$ 
denote the element whose $\operatorname{kernel}\left(\mathcal{D}_{C}\right)$ summand is $o$ and whose other summands are zero. Given that any element $o \in \operatorname{kernel}\left(\mathcal{D}_{C}\right)$ obeys $|o| \leq c_{0} e^{-|s| / c_{0}}\|o\|_{\mathcal{L}^{2}}$, it follows that $\left\|q\left(\xi^{o}\right)-o_{V}\right\|_{\mathcal{L}^{2}} \leq c_{0} r^{-1 / c_{0}}\|o\|_{\mathcal{L}^{2}}$. A similar construction starting with an element $o$ from any of the other summands in $V_{0}$ and using a suitably cut-off function defines an element $\xi^{o} \in \mathcal{K}$ with $\left\|q\left(\xi^{o}\right)-o_{V}\right\|_{\mathcal{L}^{2}}$ obeying this same bound.

The assignment $o \rightarrow \xi^{o}$ can be viewed as defining a linear map from $V_{0}$ to $\mathcal{K}$. This map is denoted in what follows by $q^{*}$. It follows from what was just said that $\left\|q q^{*}(o)-o\right\|_{\mathcal{L}^{2}} \leq c_{0} r^{-1 / c_{0}}\|o\|_{\mathcal{L}^{2}}$ and that $\left\|q^{*}(o)\right\|_{\mathcal{K}} \leq c_{0}\|o\|_{\mathcal{L}^{2}}$ for all $o \in V_{0}$.

\section{7.g Proof of Proposition 7.2: Part 5}

This subsection and the next justify the claims that are made about $\mathcal{T}_{1}$ in items (a), (b) and (c) of the proposition's second bullet. This subsection proves items (b) and (c). The next subsection proves that $\mathcal{T}_{1}$ maps $q$ 's kernel onto $\mathcal{L}$. These next two subsections assume implicitly Constraint 4 of Section 4.b.

Consider to start the $\mathcal{L}^{2}$ norm of $\mathcal{T}_{1}(\xi)$ when $\xi \in \mathcal{K}$. Given the description of $\mathcal{T}_{1}$ in the previous subsection, it follows that

$$
\begin{aligned}
c_{0}\left\|\mathcal{T}_{1}(\xi)\right\|_{\mathcal{L}^{2}}^{2} \geq \sum_{C \in \mathcal{C}}\left(\left\|\mathcal{T}_{1}\left(\xi^{C}\right)\right\|_{\mathcal{L}^{2}}^{2}-R^{-2} c_{0}\left\|\xi^{C}\right\|_{\mathcal{L}^{2}}^{2}\right) \\
+\sum_{\gamma \in \Xi_{\Sigma-}}\left(\left\|\mathcal{T}_{1}\left(\xi^{\gamma-}\right)\right\|_{\mathcal{L}^{2}}^{2}-R^{-2} c_{0}\left\|\xi^{\gamma-}\right\|_{\mathcal{L}^{2}}^{2}\right) \\
+\sum_{\gamma \in \Xi_{\Sigma+}}\left(\left\|\mathcal{T}_{1}\left(\xi^{\gamma+}\right)\right\|_{\mathcal{L}^{2}}^{2}-R^{-2} c_{0}\left\|\xi^{\gamma+}\right\|_{\mathcal{L}^{2}}^{2}\right) .
\end{aligned}
$$

To argue for (7-47), digress for the moment and suppose that $f$ and $g$ are functions on a manifold with $|g|+R|d g| \leq c_{0}$. Write $f=g f+(1-g) f$ and set $f_{1}=g f$ and $f_{2}=(1-g) f$. The analog of (7-47) in this context is the claim that $|d f|^{2} \geq c_{0}^{-1}\left(\left|d f_{1}\right|^{2}+\left|d f_{2}\right|^{2}\right)-c_{0} R^{-2}|f|^{2}$. To see why the latter inequality holds, use the fact that $d f_{1}=d g f+g d f$ to see that $\left|\left\langle d f_{1}, d f_{2}\right\rangle\right| \leq c_{0}\left|d f_{2}\right|\left(|d f|+R^{-1}|f|\right)$. This last inequality with the equality $|d f|^{2}=\left|d f_{1}\right|^{2}+\left|d f_{2}\right|^{2}+2\left\langle d f_{1}, d f_{2}\right\rangle$ implies the desired bound on $|d f|^{2}$. The inequality (7-47) follows using a straightforward, multicomponent version of the inequality $|d f|^{2} \geq c_{0}^{-1}\left(\left|d f_{1}\right|^{2}+\left|d f_{2}\right|^{2}\right)-c_{0} R^{-2}|f|^{2}$.

Equation (7-47) is used first to obtain a lower bound for the $\mathcal{L}^{2}$ norm of $\mathcal{T}_{1}$

$$
\left\|\mathcal{T}_{1}(\xi)\right\|_{\mathcal{L}^{2}} \geq c_{0}^{-1}\|\xi\|_{\mathcal{K}_{1}^{2}} \quad \text { when } q(\xi)=0
$$

Note that the assertion made by item (c) in the second bullet of Proposition 7.2 follows directly from (7-48) given what is said about $q^{*}$ in the final paragraph of Section 7.f.

The lower bound (7-48) for the $\mathcal{L}^{2}$ norm of $\mathcal{T}_{1}$ on the kernel of $q$ will be seen to follow from (7-47) given suitable lower bounds for each $\left\|\mathcal{T}_{1}\left(\xi{ }^{C}\right)\right\|_{\mathcal{L}^{2}}$ when $q(\xi)=0$, 
and on each $\left\|\mathcal{T}_{1}\left(\xi^{\gamma \pm}\right)\right\|_{\mathcal{L}^{2}}$ for any $\xi \in \mathcal{K}$. The desired lower bounds are obtained in Part 1 of what follows. Part 2 of what follows explains how to get a lower bound for both parts of the norm that defines $\mathcal{L}$.

Part 1 This part of the subsection derives lower bounds for each $\left\|\mathcal{T}_{1}\left(\xi \xi^{C}\right)\right\|_{\mathcal{L}^{2}}$ when $q(\xi)=0$, and on each $\left\|\mathcal{T}_{1}\left(\xi^{\gamma \pm}\right)\right\|_{\mathcal{L}^{2}}$ for any $\xi \in \mathcal{K}$. These lower bounds make (7-48) a direct consequence of (7-47). There are two steps in what follows.

Step 1 Suppose that $C \in \mathcal{C}$ is not $\mathbb{R}$-invariant. It follows from the description of $\mathcal{T}_{1}$ given in the previous subsection that

$$
\left\|\mathcal{T}_{1}\left(\xi^{C}\right)\right\|_{\mathcal{L}^{2}} \geq c_{0}^{-1}\left\|\mathcal{D}_{C} \xi_{C}^{C}\right\|_{L^{2}}-c_{0} r^{-1 / 2+8 \sigma}\left\|\xi_{C}^{C}\right\|_{\mathcal{K}_{1}^{2}}
$$

Meanwhile, the fourth constraint in Section 4.b implies that $\left\|\mathcal{D}_{C} \xi_{C}^{C}\right\|_{L^{2}} \geq c_{0}^{-1}\left\|\xi_{C}^{C}\right\|_{\mathcal{K}_{1}^{2}}$ when the component of $q(\xi)$ in $\operatorname{kernel}\left(\mathcal{D}_{C}\right)$ is zero. For such $\xi$,

$$
\left\|\mathcal{T}_{1}\left(\xi^{C}\right)\right\|_{\mathcal{L}^{2}}^{2}-R^{-1} c_{0}\left\|\xi^{C}\right\|_{\mathcal{L}^{2}}^{2} \geq c_{0}^{-1}\left\|\xi^{C}\right\|_{\mathcal{K}_{1}^{2}}^{2}
$$

if $R \geq c_{0}^{-1}$.

As an aside, suppose that $o \in \operatorname{kernel}\left(\mathcal{D}_{C}\right)$ and define $q^{*}(o)$ as done at the end of the previous subsection. Very much the same argument that leads to (7-47) and (7-49) finds $\left\|\mathcal{T}_{1}\left(q^{*}(o)\right)\right\|_{\mathcal{L}^{2}} \leq c_{0} r^{-1 / c_{0}}\|o\|_{\mathcal{L}^{2}}$.

Suppose next that $C=\mathbb{R} \times \gamma$ is a component of $\Sigma$ with associated integer $q$. Given what is said in Lemma 2.4, it follows from (7-36) in the case $q=1$ and from (7-37) in the case $q>1$ that (7-49) also holds in this case.

Step 2 This step proves a $\xi^{\gamma-}$ version of (7-49). To this end, introduce once again the notation from Section 5.c. Decompose $\xi^{\gamma-}$ as in (7-45) and it then follows that

$$
\left\|\mathcal{T}_{1}\left(\xi^{\gamma-}\right)\right\|_{\mathcal{L}^{2}}^{2} \geq c_{0}^{-1} \sum_{1 \leq k \leq N}\left(\left\|\mathcal{T}_{1}\left(\xi^{\gamma-k}\right)\right\|_{\mathcal{L}^{2}}^{2}-R^{-1} c_{0}\left\|\xi^{\gamma-k}\right\|_{\mathcal{L}^{2}}^{2}\right)
$$

As is explained next,

$$
\left\|\mathcal{T}_{1}\left(\xi^{\gamma-k}\right)\right\|_{\mathcal{L}^{2}} \geq c_{0}^{-1}\left\|\xi^{\gamma-k}\right\|_{\mathcal{K}_{1}^{2}} \text { when } q(\xi)=0 .
$$

This last inequality, (7-50), their $\gamma_{+}$analogs and (7-49) imply, via (7-47), the desired inequality that is asserted by (7-48).

To start the proof of (7-51), reintroduce the map $\mathfrak{c}_{k 0}: \mathbb{R} \times S^{1} \rightarrow \mathfrak{C}_{m_{k}}$ from the previous subsection. Keep in mind that the distance in $\mathfrak{C}_{m_{k}}$ between $\left.\mathfrak{c}_{k}\right|_{(w, t)}$ and $\left.\mathfrak{c}_{k 0}\right|_{(w, t)}$ is bounded by $c_{0} r^{-\sigma / 4}$ at each $(w, t) \in I_{k} \times S^{1}$. This last remark plus (7-38) imply the following: Suppose that $\zeta$ is a compactly supported, smooth section over 
$I_{k} \times S^{1}$ of $\mathfrak{c}_{k}^{*} T_{1,0} \mathfrak{C}_{m_{k}}$. Use $\exp _{\mathfrak{c}_{k 0}}$ from Section 4.b to view $\zeta$ and the $\mathfrak{c}_{k}^{*} T_{1,0} \mathfrak{C}_{m_{k}}$ component of $\mathcal{T}_{1}(\zeta)$ as a section of $\mathfrak{c}_{k 0}^{*} T_{1,0} \mathfrak{C}_{m_{k}}$ over $I_{k} \times S^{1}$. Meanwhile, the pair $(\zeta, 0) \in \mathfrak{c}_{k}^{*} T_{1,0} \mathfrak{C}_{m_{k}} \oplus\left(\bigoplus_{\mathcal{E} \in \mathcal{E}_{\gamma-}-E_{k}} \pi_{*}\left(\left.N\right|_{\mathcal{E}}\right)\right)$ defines an element in $\mathcal{K}$. Use $\zeta$ to denote the latter also. Then

$$
\mathcal{T}_{1}(\zeta)=\bar{\partial}_{\mathfrak{c}_{k 0}} \zeta+\left.\left(\nabla_{\zeta_{\mathbb{R}}} \nabla^{1,0} h\right)\right|_{\mathfrak{c}_{k 0}}+\mathfrak{e}^{\prime}
$$

where $\left\|\mathfrak{e}^{\prime}(\zeta)\right\|_{L^{2}} \leq c_{0} r^{-\sigma / 4}\|\zeta\|_{\mathcal{K}_{1}^{2}}$.

Granted (7-52), then (7-51) follows as a corollary to Lemma 7.5. Indeed, trivial cokernel or not, the fact that Lemma 7.5's operator is Fredholm has the following implication: Let $\zeta$ denote an $L_{1}^{2}$ section over $\mathbb{R} \times S^{1}$ of $\mathfrak{c}_{k 0}^{*} T^{1,0} \mathfrak{C}_{m_{k}}$

$$
\left\|\bar{\partial}_{\mathfrak{c}_{k 0}} \zeta+\left.\left(\nabla_{\zeta_{\mathbb{R}}} \nabla^{1,0} h\right)\right|_{\mathfrak{c}_{k 0}}\right\|_{L^{2}} \geq c_{0}^{-1}\|\zeta\|_{L_{1}^{2}}
$$

when $\zeta$ is $L^{2}$ orthogonal to kernel of the operator $\zeta \rightarrow \bar{\partial}_{\mathfrak{c}_{k 0}} \zeta+\left.\left(\nabla_{\zeta_{\mathbb{R}}} \nabla^{1,0} h\right)\right|_{\mathfrak{c}_{k 0}}$.

Part 2 This part uses (7-48) to prove that $\left\|\mathcal{T}_{1}(\xi)\right\|_{\mathcal{L}} \geq c_{0}^{-1}\|\xi\|_{\mathcal{K}}$ when $q(\xi)=0$. This bound with the remarks in the final paragraph of Section 7.f imply item (b) of the second bullet of Proposition 7.2.

Start the proof that $\left\|\mathcal{T}_{1}(\xi)\right\|_{\mathcal{L}} \geq c_{0}^{-1}\|\xi\|_{\mathcal{K}}$ when $q(\xi)=0$ by writing $\xi$ as in (7-44). The description of $\mathcal{T}_{1}$ in Section 7.e implies that

$$
\left\|\mathcal{T}_{1}\left(\xi^{C}\right)\right\|_{\mathcal{L}} \geq c_{0}^{-1}\left\|\mathcal{D}_{\Sigma} \xi_{C}^{C}\right\|_{\mathcal{L}}-c_{0} r^{-1 / 2+8 \sigma}\left\|\xi_{C}^{C}\right\|_{\mathcal{K}}
$$

and so an appeal to Lemma 2.7 finds that $\left\|\mathcal{T}_{1}\left(\xi^{C}\right)\right\|_{\mathcal{L}} \geq c_{0}^{-1}\left\|\xi_{C}^{C}\right\|_{\mathcal{K}} \geq c_{0}^{-1}\left\|\xi^{C}\right\|_{\mathcal{K}}$ if $q(\xi)=0$. Meanwhile, decompose each $\gamma \in \Xi_{\Sigma-}$ version of $\xi^{\gamma-}$ as in (7-47). Given (7-52) and (7-53), it again follows using Lemma 2.7 that $\left\|\mathcal{T}_{1}\left(\xi_{k}\right)\right\|_{\mathcal{L}} \geq c_{0}^{-1}\left\|\xi_{k}\right\|_{\mathcal{K}}$ when $q(\xi)=0$. Granted these bounds and (7-48), the argument that led to (7-47) finds that $\left\|\mathcal{T}_{1}\left(\xi^{\gamma-}\right)\right\|_{\mathcal{L}} \geq c_{0}^{-1}\left\|\xi^{\gamma-}\right\|_{\mathcal{K}}$ when $q(\xi)=0$. One more application of the argument for (7-47) and (7-48) finds that $\left\|\mathcal{T}_{1}(\xi)\right\|_{\mathcal{L}} \geq c_{0}^{-1}\|\xi\|_{\mathcal{K}}-c_{0} r^{-1 / 2+8 \sigma}\|\xi\|_{\mathcal{K}}$ when $q(\xi)=0$. This implies the desired inequality $\left\|\mathcal{T}_{1}(\xi)\right\|_{\mathcal{L}} \geq c_{0}^{-1}\|\xi\|_{\mathcal{K}}$ if $q(\xi)=0$ when $R>c_{0}$ and $r>c_{0}$.

\section{7.h Proof of Proposition 7.2: Part 6}

This last part of the proof explains why $\mathcal{T}_{1}$ maps the kernel of $q$ surjectively to $\mathcal{L}$. By way of an overview: The surjectivity is ultimately a consequence of the fact that each $C \in \mathcal{C}$ version of $\mathcal{D}_{C}$, and each $\gamma \in \Xi_{\Sigma \pm}$ and $k \in\left\{1, \ldots, N_{\gamma \pm}\right\}$ version of Lemma 7.5 's operator have trivial cokernel. The details are presented in three steps. 
Step 1 Write a given $\eta \in \mathcal{L}$ as

$$
\eta=\sum_{C \in \mathcal{C}} \eta^{C}+\sum_{\gamma \in \Xi_{\Sigma-}} \eta^{\gamma-}+\sum_{\gamma \in \Xi_{\Sigma+}} \eta^{\gamma+}
$$

where the decomposition here is analogous to that given in (7-44). Meanwhile, each $\gamma \in \Xi_{\Sigma-}$ version of $\eta^{\gamma-}$ is further decomposed as

$$
\eta^{\gamma-}=\eta^{\gamma-1}+\eta^{\gamma-2}+\cdots+\eta^{\gamma-N}
$$

with this one analogous to that given in (7-45). Each $y \in \Xi_{\Sigma+}$ version of $\eta^{\gamma+}$ has an analogous decomposition.

Step 2 It follows from Lemma 2.7 and Constraint 4 of Section $4 . b$ that the component $\eta_{C}^{C}$ of the term $\eta^{C}$ can be written as $\mathcal{D}_{C} \iota_{*} C$ with $\iota_{*} C$ an element in $C$ 's version of the Banach space $\mathcal{K}_{C}$. This is because $\mathcal{D}_{C}$ has trivial cokernel. Moreover, there exists a unique such element with the property that $\left(1-\sum_{\mathcal{E} \in \mathcal{E}_{C}} \chi_{\mathcal{E}}\right) \iota_{* C}$ is $L^{2}$ orthogonal to the kernel of $\mathcal{D}_{C}$. Let ${ }_{l} C$ denote the element in $\mathcal{K}$ whose components $\left.\left(\left(\iota_{C^{\prime}}^{C}\right)_{C^{\prime} \in C},\left(\iota_{\gamma-}^{C}\right)_{\gamma \in \Xi_{\Sigma-}}\right),\left(\iota_{\gamma+}^{C}\right)_{\gamma \in \Xi_{\Sigma+}}\right)$ are as follows: First, $\iota_{C}^{C}=$ $\left(1-\sum_{\mathcal{E} \in \mathcal{E}_{C}} \chi_{\mathcal{E}}^{\prime}\right) \iota_{* C}$ where $\chi_{\mathcal{E}}^{\prime}$ is the function on $C$ with compact support in $\mathcal{E}$ and given on $\mathcal{E}$ by the restriction of $\chi\left(\left(R_{\mathcal{E}}+3.5 R-|w|\right) / R\right)$. Note in particular that $\chi_{\mathcal{E}}^{\prime}=1$ where $d \chi_{\mathcal{E}} \neq 0$. The remaining components of ${ }_{\iota}{ }^{C}$ are determined by $\iota_{C}^{C}$ using (5-12).

Lemma 2.7 and Lemma 7.5 have the following implication concerning any given $\eta^{\gamma-k}$ : This section of $\mathfrak{c}_{k 0}^{*} T_{1,0} \mathfrak{C}_{m_{k}}$ can be written as $\bar{\partial}_{k 0} \iota_{k}+\left.\left(\nabla_{\iota_{k \mathbb{R}}} \nabla^{1,0} \hat{h}\right)\right|_{\mathfrak{c}_{k 0}}$ where $\iota_{k}$ has finite $K$-norm. Define

$$
\iota^{\gamma-k}=\left(\left(\iota_{C}^{\gamma-k}\right)_{C \in \mathcal{C}},\left(\iota_{\gamma^{\prime}-}^{\gamma-k}\right)_{\gamma^{\prime} \in \Xi_{\Sigma-}},\left(\iota_{\gamma^{\prime}+}^{\gamma-k}\right)_{\gamma^{\prime} \in \Xi_{\Sigma+}}\right) \in \mathcal{K}
$$

as follows: First, only the components $\iota_{\gamma-}^{\gamma-k}$ and $\left\{\iota_{C}^{\gamma-k}\right\}_{C \in \mathcal{C}}$ are nonzero, and $\iota_{C}^{\gamma-k} \neq 0$ only if $C$ intersects $U_{\gamma-}$. Second, $\iota_{\gamma-}^{\gamma-k}$ has support on the subcylinder $I_{k} \times S^{1}$ where it is given in the notation from Section 5.c as $\iota_{\gamma-}^{\gamma-k}=\left(\chi_{k}^{\prime} \iota_{k}, 0\right)$, with $\chi_{k}^{\prime}: I_{k} \rightarrow[0,1]$ defined as follows: As in (7-46), let $R_{k}$ denote the value of $R_{\mathcal{E}}$ for the ends $\mathcal{E} \in E_{k}$. Then

$$
\chi_{k}^{\prime}=\chi\left(\left(R_{k}+1.5 R-|w|\right) / R\right)\left(1-\chi\left(\left(R_{k-1}+2.5 R-|w|\right) / R\right) .\right.
$$

Note in this regard that $x_{k}^{\prime}=1$ where the differential of the (7-46)'s function $\chi_{k}$ is nonzero. The component $\iota_{C}^{\gamma-k}$ of $\iota^{\gamma-k}$ is determined from $\iota_{\gamma^{\prime}}^{\gamma-k}$, using (5-12).

Define $\iota^{\gamma-}=\sum_{1 \leq k \leq N} \iota^{\gamma-k}$. Make the analogous construction of $\iota^{\gamma+}$ for each $\gamma \in$ $\Xi_{\Sigma+}$. Then define $\iota=\iota(\eta)$ to be the sum $\iota=\sum_{C \in \mathcal{C}^{l}}{ }^{C}+\sum_{\gamma \in \Xi_{\Sigma-}} \iota^{\gamma-}+\sum_{\gamma \in \Xi_{\Sigma+}} \iota^{\gamma+}$. Note that $\mathfrak{q}(\iota)=0$; this is a consequence of the fact that any given version of $1-\chi_{\mathcal{E}}$ has support where $1-\chi_{\mathcal{E}}^{\prime}=1$, and any given version of $\chi_{k}$ has support where the corresponding $\chi_{k}^{\prime}=1$. 
Step 3 It follows from what just said and from the assertions in Lemma 2.7 and Lemma 7.5 that the assignment $\eta \rightarrow \iota(\eta)$ defines a bounded, linear map from $\mathcal{L}$ to the kernel of $q$ with norm bound $\|\iota(\eta)\|_{\mathcal{K}} \leq c_{0}\|\eta\|_{\mathcal{L}}$. This understood, it is a consequence of the descriptions of $\mathcal{T}_{1}$ in preceding subsections, and from (2-4)-(2-5), that

$$
\left\|\mathcal{T}_{1}(\iota(\eta))-\eta\right\|_{\mathcal{L}} \leq c_{0}\left(R^{-1}+r^{-1 / 2+8 \sigma}\right)\|\eta\|_{\mathcal{L}}
$$

This equation says that $\iota$ is nearly an inverse on the kernel of $\mathfrak{q}$ to the map $\mathcal{T}_{1}$ when $R$ and $r$ are large.

Fix $\eta_{*} \in \mathcal{L}$. To obtain $\xi_{*} \in \operatorname{kernel}(q)$ with $\mathcal{T}_{1}\left(\xi_{*}\right)=\eta_{*}$, define a map $\mathcal{F}: \mathcal{L} \rightarrow \mathcal{L}$ by setting

$$
\mathcal{F}\left(\eta^{\prime}\right)=-\left(\mathcal{T}_{1}\left(\iota\left(\eta_{*}+\eta^{\prime}\right)\right)-\eta_{*}-\eta^{\prime}\right) .
$$

A fixed point, $\eta$, of this linear map obeys $\mathcal{T}_{1}\left(\iota\left(\eta_{*}+\eta\right)\right)=\eta_{*}$ and so $\xi_{*}=\iota\left(\eta_{*}+\eta\right) \in$ $\operatorname{kernel}(q)$ is mapped to $\eta_{*}$ by $\mathcal{T}_{1}$. It follows from (7-57) that the map $\mathcal{F}$ is a contraction mapping when $R$ and $r$ are greater than some constant $c_{0}$. When such is the case, the map $\mathcal{F}$ has a unique fixed point in $\mathcal{L}$.

\section{7.i Proof of Lemma 7.5}

The arguments below treat the case when $(\gamma, m) \in \Xi_{\Sigma-}$. Except for changing some signs and changing "greatest lower" to "least upper", the same arguments will prove the lemma for the cases when $(\gamma, m) \in \Xi_{\Sigma+}$. The details for the latter cases are omitted. This understood, fix $(\gamma, m) \in \Xi_{\Sigma-}$ and $k \in\left\{1, \ldots, N_{\gamma-}\right\}$. The proof that Lemma 7.5's operator is Fredholm with trivial cokernel has four steps.

Step 1 The operator in question has a relatively simple form in the cases at hand when written in terms of the coordinates in (1-5). If $m_{k}=1$, these coordinates identify this operator with that on $C^{\infty}\left(\mathbb{R} \times S^{1} ; \mathbb{C}\right)$ that sends a given function $\zeta$ to $\bar{\partial} \zeta+v \zeta+\mu \bar{\zeta}$. The $m_{k}=1$ version of Lemma 7.5 follows directly from this identification given that (1-2) lacks a zero eigenvalue.

In the case $m_{k}>1$, the operator in question is best viewed by using the functions in (1-5) to identify $\mathfrak{C}_{m_{k}}$ with $\mathbb{C}^{m_{k}}$ and so use the basis $\left\{\partial / \partial \sigma_{q}\right\}_{1 \leq q \leq m_{k}}$ to view a section of $\mathfrak{c}_{k 0}^{*} T_{1,0} \mathfrak{C}_{m_{k}}$ as an element in $C^{\infty}\left(\mathbb{R} \times S^{1} ; \mathbb{C}^{m_{k}}\right)$. Viewed in this light, Lemma 7.5 's operator acts diagonally with the $q$-th diagonal entry given by $\bar{\partial}+(q / 2) \mathrm{R}$ where $\mathrm{R}$ is the rotation number of $\gamma$. The constraints in Section 4.b guarantee that 0 is not an eigenvalue of the operator $(i / 2)(d / d t)+(q / 2) \mathrm{R}$ on $L^{2}\left(S^{1} ; \mathbb{C}\right)$. This implies that $\bar{\partial}+(q / 2) \mathrm{R}$ is Fredholm as a map from $L_{1}^{2}\left(\mathbb{R} \times S^{1} ; \mathbb{C}\right)$ to $L^{2}\left(\mathbb{R} \times S^{1} ; \mathbb{C}\right)$. Even so, the claim asserted in Lemma 7.5 does not immediately follow because the metric on 
$\mathfrak{C}_{m_{k}}$ is not the induced metric from $\mathbb{C}^{m_{k}}$ unless $k=N$ and the one end in $U_{\gamma-}$ is the $w \ll-1$ part of $\mathbb{R} \times \gamma$. Thus, more work need be done.

Step 2 The simplest case to analyze is that where $\mathcal{E}_{\gamma-}=\{\mathcal{E}\}$ and so $m_{\gamma-}=q_{\mathcal{E}}$. Assume that $\mathcal{E}$ is not the $w \ll-1$ part of $\mathbb{R} \times \gamma$. In this case, $k=1$ and $m_{k}=q_{\mathcal{E}}$. The norm that is induced on the trivial bundle $\left(\mathbb{R} \times S^{1}\right) \times \mathbb{C}^{q_{\mathcal{E}}}$ by the isomorphism with the pullback via $\mathfrak{c}_{10}$ of $T_{1,0} \mathfrak{C}_{q_{\mathcal{E}}}$ is diagonal. This follows by virtue of the fact that the Riemannian metric on $\mathfrak{C}_{q_{\mathcal{E}}}$ is invariant with respect to the $S^{1}$ action that is induced by the standard action of $S^{1}$ on $\mathbb{C}$. Meanwhile, it follows from (2-1) and (2-2) that the inner product between the dual basis elements $\left\{d \sigma_{q}\right\}_{1 \leq q \leq q_{\mathcal{E}}}$ for the $\mathfrak{c}_{10}$ pullback of $T^{1,0} \mathfrak{C}_{q_{\mathcal{E}}}$ has the form

$$
\left\langle d \sigma_{q}, d \sigma_{q}\right\rangle=c_{*} \rho^{2 q-2} q^{2}\left|\mathcal{u}_{\mathcal{E}}\right|^{2}+\mathfrak{m}_{q},
$$

where the notation is as follows: First, $c_{*}>0$ is a constant that depends only on $q_{\mathcal{E}}$. Second, $\rho=\rho(w)$ is shorthand for $r_{\gamma}^{1 / 2} e^{-\left(\mathrm{R}-k_{\mathcal{E}} / q_{\mathcal{E}}\right) w}$ where $k_{\mathcal{E}}$ is the smallest integer such that $k_{\mathcal{E}} / q_{\mathcal{E}}>\mathrm{R}$. Note here that the eigenvalue $\lambda_{q_{\mathcal{E}}}$ is equal to $\frac{1}{2}\left(\mathrm{R}-k_{\mathcal{E}} / q_{\mathcal{E}}\right)$. Third, $u_{\mathcal{E}} \in \mathbb{C}-0$ is a constant. Third, $\left|\mathfrak{m}_{q}\right| \leq c_{0}$ and $\mathfrak{m}_{q}$ is asymptotic as $w \rightarrow-\infty$ to a positive constant.

With it understood that $\left(\mathbb{R} \times S^{1}\right) \times \mathbb{C}^{q_{\mathcal{E}}}$ is the $\mathfrak{c}_{10}$ the pullback of $T_{1,0} \mathfrak{C}_{q_{\mathcal{E}}}$, write an element in the $q$-th summand of $\left(\mathbb{R} \times S^{1}\right) \times \mathbb{C}^{q_{\mathcal{E}}}$ as $\eta(1+\rho)^{q-1}\left(\partial / \partial \sigma_{q}\right)$. It follows from (7-59) that this element has norm $\mathfrak{n}_{q}|\eta|$ where $c_{0}^{-1} \leq \mathfrak{n}_{q} \leq c_{0}$ and where $\mathfrak{n}_{q}$ has constant limits as $s \rightarrow \pm \infty$. Meanwhile, the operator $\bar{\partial}+(q / 2) \mathrm{R}$ acts on such an element so as to send $\eta$ to

$$
\bar{\partial} \eta+\frac{1}{2}\left(q \mathrm{R}+(q-1) \rho /(1+\rho)\left(k_{\mathcal{E}} / q_{\mathcal{E}}-\mathrm{R}\right)\right) \eta .
$$

This operator looks like

- $\bar{\partial}+\frac{q}{2} \mathrm{R}$ for $w \ll-1$.

- $\bar{\partial}+\frac{1}{2}\left(\mathrm{R}+(q-1) k_{\mathcal{E}} / q_{\mathcal{E}}\right)$ for $w \gg 1$.

Given the Constraint 1 in Section 6.a, what is written in (7-61) implies that (7-60) is Fredholm from $L_{1}^{2}\left(\mathbb{R} \times S^{1} ; \mathbb{C}\right)$ to $L^{2}\left(\mathbb{R} \times S^{1} ; \mathbb{C}\right)$ when the norm on $\mathbb{C}$ sends $\eta$ to $\mathfrak{n}_{q}|\eta|$. Indeed, this is so because neither of the operators

$$
\frac{i}{2} \frac{d}{d t}+\frac{1}{2} \mathrm{R} \quad \text { or } \quad \frac{i}{2} \frac{d}{d t}+\frac{1}{2}\left(\mathrm{R}+(q-1) k_{\mathcal{E}} / q_{\mathcal{E}}\right)
$$

on $L^{2}\left(S^{1} ; \mathbb{C}\right)$ have eigenvalue 0 . Moreover the fact that $k_{\mathcal{E}} / q_{\mathcal{E}}>\mathrm{R}$ implies that the spectral flow from the left most to the right most operator in (7-62) is positive. This 
implies that (7-60) has nonnegative index. The fact that $k_{\mathcal{E}} / q_{\mathcal{E}}>\mathrm{R}$ also implies that the cokernel is trivial. To see this, note first that the $L^{2}$ adjoint of (7-60) annihilates only linear combinations of functions that have the form $(w, t) \rightarrow e^{u(w)-k(w-i t)}$ where $k \in \mathbb{Z}$, and where the function $u(\cdot)$ obeys

- $u(w)=q \mathrm{R}+o(1)$ as $w \rightarrow-\infty$.

- $u(w)=\left(\mathrm{R}+(q-1) k_{\mathcal{E}} / q_{\mathcal{E}}\right) w+o(1)$ as $w \rightarrow \infty$.

These asymptotics for $u$ follow from (7-61). Meanwhile, it follows from (7-63) that the function $(w, t) \rightarrow e^{u(w)-k(w-i t)}$ is an $L^{2}$ function if and only if $k<q \mathrm{R}$ and $k>\mathrm{R}+(q-1) k_{\mathcal{E}} / q_{\mathcal{E}}$. As there are no such integers, (7-60) has trivial cokernel.

Step 3 The second case to consider is that when all of the ends in $\mathcal{E}_{\gamma-}$ have the same degree. Thus, $q_{\mathcal{E}}=q_{\mathcal{E}^{\prime}}$ for any pair $\mathcal{E}$ and $\mathcal{E}^{\prime}$ from $\mathcal{E}_{\gamma-}$ and $m_{\gamma-}=n q_{\mathcal{E}}$ with $\mathcal{E}$ any end from $\mathcal{E}_{\gamma-}$. Assume that $n>1$. In this case, $\mathcal{E}_{\gamma-}=\mathcal{E}_{1}$ and the metric that is induced on the vector bundle $\left(\mathbb{R} \times S^{1}\right) \times \mathbb{C}^{n q_{\mathcal{E}}}=c_{10}^{*} T_{1,0} \mathfrak{C}_{n q_{\mathcal{E}}}$ is not diagonal. The configuration $\mathfrak{Z}_{\mathcal{E}_{0}}$ that is labeled by any given $\mathcal{E} \in \mathcal{E}_{\gamma-}$ is determined by a function of the form

$$
z_{\mathcal{E} 0}=u_{\mathfrak{E}} e^{-\left(\mathrm{R}-k_{\mathfrak{E}} / q_{\mathfrak{E}}\right) w+i\left(k_{\mathcal{E}} / q_{\mathfrak{E}}\right) t},
$$

where $u_{\mathcal{E}} \in \mathbb{C}-\{0\}$. Moreover, Constraint 3 in Section 4.b asserts that $u_{\mathcal{E}} \neq \eta u_{\mathcal{E}^{\prime}}$ with $\eta$ a $q_{\mathcal{E}}$-th root of unity if $\mathcal{E} \neq: \mathcal{E}^{\prime}$. It follows from (7-64) and (2-1)-(2-2) that the inner product between the $\mathfrak{c}_{10}$ pullbacks of the dual basis elements $\left\{d \sigma_{q}\right\}_{1 \leq q \leq n q_{\mathcal{E}}}$ for $T^{1,0} \mathfrak{C}_{n q_{\mathcal{E}}}$ are such that $\left\langle d \sigma_{q}, d \sigma_{q^{\prime}}\right\rangle=0$ when $q \neq q^{\prime} \bmod \left(q_{\mathcal{E}}\right)$. Meanwhile, the inner product of $d \sigma_{q}$ with $d \sigma_{q^{\prime}}$ when $q=q^{\prime} \bmod \left(q_{\mathcal{E}}\right)$ can be written as

$$
\left\langle d \sigma_{q}, d \sigma_{q^{\prime}}\right\rangle=c_{*} \rho^{q+q^{\prime}-2} q q^{\prime} e^{i\left(q^{\prime}-q\right)\left(k_{\mathfrak{E}} / q_{\mathscr{E}}\right) t} \sum_{\mathcal{E}, \mathcal{E}^{\prime} \in \mathcal{E}_{\gamma-}} \bar{u}_{\mathcal{E}}^{q-1} u_{\mathcal{E}^{\prime}}^{q^{\prime}-1}+\mathfrak{m}_{q q^{\prime}}
$$

Here, $\left|\mathfrak{m}_{q q^{\prime}}\right| \leq c_{0}$. Furthermore, $\mathfrak{m}_{q q^{\prime}}$ limits as $w \rightarrow-\infty$ to zero unless $q=q^{\prime}$. In the latter case, it limits to a positive constant. Identify the pullback by $\mathfrak{c}_{10}$ of the $(1,0)$ tangent bundle as $\left(\mathbb{R} \times S^{1}\right) \times \mathbb{C}^{n q_{E}}$ and define $\rho(w)$ as before so as to write the $q$-th summand of this bundle as $\eta_{q}(1+\rho)^{q-1}\left(\partial / \partial \sigma_{q}\right)$. By virtue of (7-65), the induced inner product between vectors $n=\left(\eta_{q}\right)_{1 \leq q \leq n q_{\mathcal{E}}}$ and $n^{\prime}=\left(\eta_{q}^{\prime}\right)_{1 \leq q \leq n q_{\mathcal{E}}}$ can be written as

$$
\left\langle n, n^{\prime}\right\rangle=\sum_{1 \leq q, q^{\prime} \leq n q_{\mathcal{E}}} g_{q, q^{\prime}} \bar{\eta}_{q} \eta_{q^{\prime}}^{\prime},
$$

where the matrix $g$ with $\left(q, q^{\prime}\right)$ entry $g_{q, q^{\prime}}$ has constant, positive definite limits as $w \rightarrow-\infty$ and also as $w \rightarrow \infty$. Indeed, the $w \rightarrow-\infty$ limit of $g$ is diagonal with $q$-th entry $\mathfrak{m}_{q, q}$. Meanwhile, the $w \rightarrow \infty$ limit has $\left(q, q^{\prime}\right)$ entry zero unless $q=q^{\prime} \bmod \left(q_{\mathcal{E}}\right)$ in which case the entry is $\sum_{\mathcal{E}, \mathcal{E}^{\prime} \in \mathcal{E}_{\gamma-}} \bar{u}_{\mathcal{E}}^{q-1} u_{\mathcal{E}^{\prime}}^{q^{\prime}-1}$. These entries define a nondegenerate, positive definite matrix by virtue of the constraint that $\mathcal{u}_{\mathcal{E}} \neq \eta \mathcal{u}_{\mathcal{E}^{\prime}}$ if $\mathcal{E} \neq \mathcal{E}^{\prime}$ and $\eta^{q_{\mathcal{E}}}=1$. 
In terms of $\eta_{q}$, the operator $\bar{\partial}+(q / 2) \mathrm{R}$ acts as depicted in (7-60) and so (7-61) still holds. The latter implies that Lemma 7.5's operator is Fredholm with nonnegative index and trivial cokernel. The argument is that used for Step 2's version of this assertion.

Step 4 Consider now the general case. Let $n$ denote the number of ends of $\Sigma$ in $\mathcal{E}_{k}$, and write $m_{k}=n q_{\mathcal{E}}+m_{k-1}$ where $q_{\mathcal{E}}$ is the integer degree that is defined by the ends from $\mathcal{E}_{k}$. Introduce the complex number $\boldsymbol{u}_{\mathcal{E}}$ for $\mathcal{E} \in \mathcal{E}_{k}$ as defined in (7-64). These are nonzero numbers, and they are such that $u_{\mathcal{E}} \neq \eta u_{\mathcal{E}^{\prime}}$ if $\mathcal{E} \neq: \mathcal{E}^{\prime}$ and $\eta^{q_{\mathcal{E}}}=1$.

As before, the inner product $\left\langle d \sigma_{q}, d \sigma_{q^{\prime}}\right\rangle$ is asymptotic as $w \rightarrow-\infty$ to zero if $q \neq q^{\prime}$ and to a positive constant if $q=q^{\prime}$. It is a consequence of (2-1) and (2-2) that the $w \gg 1$ version of this inner product has the form

$$
\left\langle d \sigma_{q}, d \sigma_{q^{\prime}}\right\rangle=c_{*} \rho^{q+q^{\prime}-2} q q^{\prime} e^{i\left(q^{\prime}-q\right)\left(k_{\mathcal{E}} / q_{\mathscr{E}}\right) t} \sum_{\mathcal{E}, \mathcal{E}^{\prime} \in \mathcal{E}_{k}} \bar{u}_{\mathcal{E}}^{q-1} u_{\mathcal{E}^{\prime}}^{q^{\prime}-1}+\mathfrak{m}_{q q^{\prime}}
$$

where $c_{*}=0$ when $q \neq q^{\prime} \bmod \left(q_{\mathcal{E}}\right)$ and is a positive constant when $q=q^{\prime} \bmod \left(q_{\mathcal{E}}\right)$. Meanwhile, $\mathfrak{m}_{q, q^{\prime}}$ has bounded norm and it is asymptotic as $w \rightarrow \infty$ to zero unless $q=q^{\prime}$. In the latter case, it has a positive $w \rightarrow \infty$ limit. There is one other crucial point to make here: The matrix $m_{k-1} \times m_{k-1}$ matrix with $\left(q, q^{\prime}\right) \in \times_{2}\left\{1, \ldots, m_{k-1}\right\}$ entry $\mathfrak{m}_{q, q^{\prime}}$ is positive definite at large $w$. This is because the $w \rightarrow \infty$ limit of this matrix gives the inner product on the fiber of $T^{1,0} \mathfrak{C}_{m_{k-1}}$ at the symmetric vortex for the basis $\left\{d \sigma_{q}\right\}_{1 \leq q \leq m_{k-1}}$.

Fix $m \in\left\{1, \ldots, q_{\mathcal{E}}\right\}$. The $n \times n$ matrix $\mathfrak{g}_{m}$ whose $\left(p, p^{\prime}\right)$ entry is

$$
\sum_{\mathcal{E}, \mathcal{E}^{\prime} \in \mathcal{E}_{\gamma-}}\left(\overline{\mathfrak{u}}_{\mathcal{E}^{\prime}} \mathfrak{u}_{\mathcal{E}^{\prime}}\right)^{m-1} \overline{\mathfrak{u}}_{\mathfrak{E}}^{(p-1) q_{\mathcal{E}}} \mathfrak{u}_{\mathcal{E}^{\prime}}^{\left(p^{\prime}-1\right) q_{\mathcal{E}}}
$$

is nondegenerate by virtue of the fact that there are $n$ ends in $\mathcal{E}_{k}$ and no $\mathfrak{u}_{\mathcal{E}}$ can be written as $\eta \mathfrak{u}_{\mathcal{E}^{\prime}}$ where $\mathcal{E}^{\prime}$ is also in $\mathcal{E}_{k}$ and where $\eta^{q_{\mathcal{E}}}=1$. This understood, let $m_{k, m}$ denote the number of integers in $\left\{1, \ldots, m_{k}\right\}$ that equal $m \bmod \left(q_{\mathcal{E}}\right)$. It follows from what was just said about $\mathfrak{g}_{m}$ that the $m_{k, m} \times m_{k, m}$ matrix whose $\left(p, p^{\prime}\right)$ entry is

$$
\sum_{\mathcal{E}, \mathcal{E}^{\prime} \in \mathcal{E}_{\gamma-}}\left(\overline{\mathfrak{u}}_{\mathfrak{E}^{-} \mathfrak{u}_{\mathcal{E}^{\prime}}}\right)^{m-1} \overline{\mathfrak{u}}_{\mathcal{E}}^{(p-1) q_{\mathcal{E}}} \mathfrak{u}_{\mathcal{E}^{\prime}}^{\left(p^{\prime}-1\right) q_{\mathcal{E}}}
$$

has kernel dimension $m_{k, m}-n$. Moreover, this kernel has a basis whose $k$-th element has $k$-th entry in $\mathbb{C}^{m_{k, n}}$ equal to 1 and all $k^{\prime}<k$ entries equal to zero.

Granted this last point, write $m_{k}=m_{k-1}+n q_{\mathcal{E}}$ and then identify $\mathfrak{c}_{k 0}^{*} T_{1,0} \mathfrak{C}_{m_{k}}$ with $\left(\mathbb{R} \times S^{1}\right) \times \mathbb{C}^{m_{k-1}+n q_{\mathcal{E}}}$ in the usual way. Then the vector space $\mathbb{C}^{m_{k-1}+n q_{\mathcal{E}}}$ has a basis of the following sort: The $q$-th basis vector in the case that $q \leq m_{k-1}$ is 
$\iota_{q}=\partial / \partial \sigma_{q}$. Meanwhile, the $q$-th basis vector when $q>m_{k-1}$ has the form

$$
\begin{aligned}
\iota_{q}= & (1+\rho)^{q-1} \\
& \times\left(\frac{\partial}{\partial \sigma_{q}}+\sum_{\substack{q^{\prime}: q^{\prime}<q, q^{\prime}=q \bmod \left(q_{\mathcal{E}}\right)}} c_{q q^{\prime}}\left(1+\rho^{\left(q^{\prime}-q\right)}\right)^{-2} \rho^{\left(q^{\prime}-q\right)} e^{-i\left(q^{\prime}-q\right)\left(k_{\mathcal{E}} / q_{\mathcal{E}}\right) t} \frac{\partial}{\partial \sigma_{q^{\prime}}}\right),
\end{aligned}
$$

where $c_{q q^{\prime}}$ is independent of $w$ and $t$. This basis is such that the numbers

$$
\left\langle\iota_{q}, \iota_{q^{\prime}}\right\rangle=g_{q q^{\prime}}
$$

are the components of a matrix whose $w \rightarrow \pm \infty$ limits define a positive definite matrix.

The operator in Lemma 7.5 does not act diagonally with respect to this basis; it acts as a lower triangular matrix whose entries on the diagonal are such as to send $\eta \iota_{q}$ to what is written in (7-60) in the case $q>m_{k-1}$, and to $\bar{\partial} \eta+(q / 2) \mathrm{R} \eta$ in the case when $q \leq m_{k-1}$. This understood, it again follows directly from (7-61) using the arguments from Step 2 that Lemma 7.5's is Fredholm with trivial cokernel.

\section{7.j Loose ends}

Proposition 6.4 and Proposition 7.1 describe a pair $(\xi, \mathfrak{b}=(b, \eta, \phi))$ that has Properties 1 and 2 from Section 5.d. Thus $(\xi, \mathfrak{b})$ obeys (5-20). Define $(A, \psi)$ using (5-19). The next proposition asserts that $(A, \psi)$ is gauge equivalent to an instanton solution of (1-13).

Proposition 7.6 There exists $\kappa \geq 1$ with the following significance: If $r \geq \kappa$, then Propositions 6.4 and 7.1 can be invoked using any given element from the radius $\kappa^{-1}$ ball in Proposition 7.1's vector space $V_{0}$ to define a pair $(\xi, \mathfrak{b}=\mathfrak{b}(\xi))$ that solves (5-20). Use this pair to define the pair $(A, \psi)$ in (5-19). The latter is smooth, and there is a smooth map $u: \mathbb{R} \times M \rightarrow S^{1}$ such that the assignment $s \rightarrow \mathfrak{d}(s)=\left.\left(A-u^{-1} d u, u \psi\right)\right|_{s}$ defines an instanton solution to (1-14) whose $s \rightarrow-\infty$ limit is the solution to (1-13) that is obtained from the data $\Theta=\Theta_{-}$using the constructions in Section 3. Meanwhile, the $s \rightarrow \infty$ limit is gauge equivalent to the solution to (1-13) that is obtained using the constructions in Section 5 from the data $\Theta=\Theta_{+}$.

Proof of Proposition 7.6 To see why $(A, \psi)$ is smooth, write $(A, \psi)$ as $\left(A^{*}+\hat{a}\right.$, $\left.\psi^{*}+\lambda\right)$. Note that $\left(A^{*}, \psi^{*}\right)$ is smooth. Let $\phi$ denote the $i \mathbb{R}$ component of $\mathfrak{b}$. Equations (2-9), (2-10) and (5-20) imply that $\mathfrak{k}=(\hat{a}, \lambda, \phi)$ obey a first order, elliptic equation with quadratic nonlinearities. Furthermore, $\mathfrak{k}$ restricts to any given radius 1 or less ball in $\mathbb{R} \times M$ as an $L_{1}^{2}$ configuration whose $L_{1}^{2}$ norm is bounded by a constant that is independent of the chosen ball. This understood, the bootstrapping techniques from [3, Chapter 6] can be employed to prove that $\mathfrak{k}$ is smooth. 
The proof that $(A, \psi)$ is gauge equivalent to an instanton solution to (1-14) invokes a result, Theorem 1.1, from Paper III of this series [9]. To start the proof, introduce $\mathfrak{c}_{-}=\left(A^{-}, \psi^{-}\right)$to denote the solution to (1-13) that is constructed in Section 3 using $\Theta=\Theta_{-}$. Write $(A, \psi)$ where $s \ll-1$ as $\left(A^{-}+\hat{a}^{-}, \psi^{-}+\lambda^{-}\right)$. Set $\mathfrak{k}^{-}=\left(\hat{a}^{-}, \lambda^{-}, \phi\right)$. Suppose that the $\mathfrak{c}=\mathfrak{c}_{-}$versions of the operator in (3-8) has trivial kernel. Granted that such is the case, arguments that are cosmetic variations of those used for [7, Lemma 4.6] can be employed to prove the following: There is a constant $\varepsilon>0$ with the following significance: Take $r \geq c_{0}$ and that $k \in\{0,1,2, \ldots\}$. Then

$$
\left|\nabla_{-}^{k_{\mathfrak{k}}-}\right| \leq c_{k} e^{-\varepsilon|s|}
$$

at points where $s \ll-1$. Here, $c_{k}$ is a constant and $\nabla_{-}$denotes the covariant derivative on sections of $i T^{*} M \oplus \mathbb{S} \oplus i \mathbb{R}$ that is defined by the Levi-Civita connection on $T(\mathbb{R} \times M)$ and the connection $A^{-}$. Let $\mathfrak{c}_{+}=\left(A^{+}, \psi^{+}\right)$denote the solution to (1-13) that is constructed in Section 3 using $\Theta=\Theta_{+}$. The same sort of argument proves the $s \gg 1$ analog of (7-72) if the $\mathfrak{c}=\mathfrak{c}_{+}$version of (3-8) has trivial kernel.

Theorem 1.1 in the third paper [9] of this series asserts that both the $\mathfrak{c}=\mathfrak{c}_{ \pm}$versions of (3-8) do indeed have trivial kernel. This understood, (7-72) and its $s \gg 1$ analog imply that the function $\phi$ and all of its derivatives converge to zero as $|s| \rightarrow \infty$ on $\mathbb{R} \times M$ at an exponential rate. This implies that

$$
\mathrm{w}(s, \cdot)=(2 r)^{1 / 2} \int_{-\infty}^{s} \phi(\sigma, \cdot) d \sigma
$$

converges to zero with all of its derivatives as $s \rightarrow-\infty$ and converges as do all of its derivatives as $s \rightarrow \infty$. Set $u: \mathbb{R} \times M \rightarrow S^{1}$ to equal $e^{\mathrm{w}}$. Then $\left(A-u^{-1} d u, u \psi\right)$ defines an instanton solution to (1-13) whose $s \rightarrow-\infty$ limit is $\mathfrak{c}_{-}$and whose $s \rightarrow \infty$ limit is gauge equivalent to $\mathfrak{c}_{+}$.

\section{7.k Proof of Theorem 1.2}

The space $\mathcal{M}_{1}\left(\Theta_{-}, \Theta_{+}\right)$has finite set of components, and each component is a smooth, 1-dimensional manifold, with any given element obtained from a unique centered element by translating a suitable constant amount along the $\mathbb{R}$ factor of $\mathbb{R} \times M$. Let $\Sigma \subset$ $\mathcal{M}_{1}\left(\Theta_{-}, \Theta_{+}\right)$denote a centered element. If $r \geq c_{0}$, then the constructions described in Sections 4-7.j assign to $\Sigma$ a solution, $\Psi^{r}(\Sigma)$ in $\mathcal{M}\left(\mathfrak{c}_{-}, \mathfrak{c}_{+}\right)$. This is the element that is obtained from Proposition 7.1 by taking $\lambda=0$. Let $\Sigma^{\prime}$ denote the result of translating $\Sigma$ by a given amount along the $\mathbb{R}$ factor of $\mathbb{R} \times M$. Define $\Psi^{r}\left(\Sigma^{\prime}\right)$ to be the translate of $\Psi^{r}(\Sigma)$ by the same factor. Given that there are but a finite number of components of $\mathcal{M}_{1}\left(\Theta_{-}, \Theta_{+}\right)$, the map $\Psi^{r}$ is defined on the whole of this space for $r \geq c_{0}$. 
A brief digression is needed to prove that $\Psi^{r}$ is $1-1$. To start the digression, fix a centered element $\Sigma \subset \mathcal{M}_{1}\left(\Theta_{-}, \Theta_{+}\right)$and write $\Psi^{r}(\Sigma)$ as in (5-19). The curvature 2-form, $F_{A}$, of $A$ has the following two properties:

- The $L^{2}$ norm of $F_{A}$ over the $|s| \leq R$ portion (5-3)'s set $U_{0}$ is bounded by $c_{0}$.

- Fix $C \in \mathcal{C}$ and let $s_{0} \in[-R+1, R-1]$. The $L^{2}$ norm of $F_{A}$ over the $s \in\left[s_{0}-1, s_{0}+1\right]$ part of (5-3)'s set $U_{C}$ is greater than $c_{0}^{-1} r^{1 / 2}$.

These points follow from the second item of Proposition 6.4.

Now suppose that $\Sigma$ and $\Sigma^{\prime}$ are distinct, centered elements in $\mathcal{M}_{1}\left(\Theta_{-}, \Theta_{+}\right)$. To see that $\Psi^{r}$ separates these two element, note first that if $r \geq c_{0}$, then there exists a component, $C^{\prime}$, of $\Sigma^{\prime}$ and $s_{0} \in[-R+1, R-1]$ such that the $s \in\left[s_{0}-1, s_{0}+1\right]$ part of $U_{C^{\prime}}$ lies in $\Sigma$ 's version of $U_{0}$. This understood, it follows from (7-74) that the $\Psi^{r}(\Sigma) \neq \Psi^{r}\left(\Sigma^{\prime}\right)$. Given that each component of $\mathcal{M}_{1}\left(\Theta_{-}, \Theta_{+}\right)$is an orbit of the $\mathbb{R}$ action, this last observation implies that the $\Psi^{r}$ image of $\Sigma$ 's component is disjoint from the component of $\Sigma^{\prime}$.

The observations in (7-74) also imply that $\Psi^{r}$ separates the points in any given component. Indeed, if this is not the case for $\Sigma$ 's component, then $\Psi^{r}(\Sigma)$ must be $\mathbb{R}$-invariant. However, this is precluded by (7-74) unless $\Sigma$ is $\mathbb{R}$-invariant.

\section{Index to the notation}

$v$ : the Reeb vector field

$a$ : the contact $1-$ form

$\ell_{\gamma}$ : the integral of the contact form a along the Reeb orbit $\gamma$

$(\nu, \mu)$

$\mathcal{L}$

hyperbolic, elliptic, $n$-elliptic

$2 \pi k$-periodic

$\mathfrak{C}_{n}$

$\sigma_{q}$

h

$\mathfrak{C}_{(\gamma, m)}$

$\mathrm{cl}, \widehat{c}, \psi^{\dagger} \tau \psi$ : Clifford multiplication maps

$\mathbb{S}$ : the spinor bundle

$K^{-1}$ : the inverse of the canonical bundle

$\nabla:$ the covariant derivative for the spinor bundle
Part 1 of Section 1.a

Part 1 of Section 1.a

Part 1 of Section 1.a

Equation (1-1)

Equation (1-2)

Part 2 of Section 1.a

Part 2 of Section 1.a

Part 1 of Section 1.b

Equation (1-5)

Equation (1-9)

Part 4 of Section 1.b

Part 1 of Section 1.c

Part 1 of Section 1.c

Part 1 of Section 1.c

Part 2 of Section 1.c 
$A_{K}:$ a chosen connection on $K^{-1}$

$\mathrm{E}$

$D_{A}$ : the Dirac operator

$B_{A}$ : the Hodge dual of the curvature 2-form a connection $A$

$\mu$ : a given 1 -form with small $C^{k}$ norms

$B_{A_{K}}$ : the Hodge dual of the curvature 2-form of the connection $A_{K}$

gauge equivalence

$\Theta$

$\mathfrak{C} \Theta, \mathfrak{C} \Theta^{*}$

$\mathcal{Z}^{L}, \mathfrak{C} \mathcal{Z}^{L}, \mathfrak{C} \mathcal{Z}^{L *}$

$\mathcal{M}_{1}\left(\Theta_{-}, \Theta_{+}\right)$

instanton

$\mathfrak{d}:$ an instanton

$\mathcal{M}\left(\mathfrak{c}_{-}, \mathfrak{c}_{+}\right)$

$\Theta$ is simple

$c_{0}$ : a constant greater than 1 that is independent of all relevant parameters. It increases between subsequent appearances.

$\vartheta_{\mathfrak{c}}$

$\vartheta_{\mathfrak{c}} \vartheta_{\mathfrak{c}}^{\dagger}$

$\varepsilon_{0}$

$\exp _{\mathfrak{c}}(\zeta)$

$v_{\zeta}$

$\nabla^{1,0} h$

$A_{0}$ in the context of a Reeb orbit

$A_{0}$ in the context of a surface

$f_{\zeta}$ in the context of a Reeb orbit

$f_{\zeta}$ in the context of a surface

$\left(x_{\zeta}, l_{\zeta}\right)$ in the context of a Reeb orbit

$\left(x_{\zeta}, \iota_{\zeta}\right)$ in the context of a surface

$\Pi_{\mathfrak{c}_{* \zeta}(\cdot)}$ in the context of a Reeb orbit

$\Pi_{\mathfrak{c}_{* \zeta}(\cdot)}$ in the context of a surface

$\mathfrak{C}_{E, n}$

$V_{1,0}$

$\|\zeta\|_{\mathcal{K}_{1}^{2}}^{2},\|\zeta\|_{\mathcal{K} *}^{2},\|\zeta\|_{\mathcal{K}}^{2}$
Part 2 of Section 1.c

Equation (1-12)

Part 2 of Section 1.c

Part 3 of Section 1.c

Part 5 of Section 1.c

Equation (1-13)

Part 5 of Section 1.c

Section 1.d

Section 1.d

Section 1.d

Section 1.e

Section 1.f

Section 1.f

Section 1.f

Equation (1-15)

Equation (2-6)

Equation (2-7)

Part 8 of Section 2.a

Equation (2-9)

Equation (2-10)

Equation (2-13)

Section 2.c, Equation (2-18).

Part 1 of Section 2.g, Equation

(2-32)

Equation (2-20)

Equation (2-39)

Equation (2-21)

Equation (2-37)

Part 2 of Section 2.d

Part 3 of Section 2.g

Section 2.e

Section 2.e

Equation (2-27) 
$\mathcal{K}_{\mathfrak{c} *}, \mathcal{K}_{\mathfrak{c}}$

$\mathfrak{L}_{\mathfrak{c}}$ with its norm defined

$\mathfrak{s}$ : the canonical section on the pullback

$\pi^{*} E \rightarrow E$

$\mathfrak{s}$ when $E$ is the normal bundle to a curve

$\theta$ : the canonical connection on $\pi^{*} E$

$\theta$ when $E$ is the normal bundle to a curve

$d^{V}, \partial^{V}, \nabla_{\theta}^{H}$

$\left(A^{\zeta}, \alpha^{\zeta}\right)$

$\mathfrak{J}=\left\{\left(\mathfrak{c}_{\gamma}, \zeta_{\gamma}\right)\right\}_{(\gamma, m)} \in \Theta$

$\rho_{*}$

$U_{0}$ in the context of Theorem 1.1

$U_{0}$ in the context of Theorem 1.2

$\left(A_{0}, \psi_{0}\right)$ in the context of Theorem 1.1

$\left(A_{0}, \psi_{0}\right)$ in the context of Theorem 1.2

$U_{\gamma}$

$\chi$ : a cut-off function chosen for eternity

$\chi_{\gamma}$

$r_{\gamma}=\left(\ell_{\gamma} /(2 \pi)\right) r$

$\hat{r}_{\gamma}$

$\left(A_{\gamma}, \psi_{\gamma}\right)$

$\mathfrak{c}_{\Theta \mathfrak{J}}$

$\mathfrak{L}_{\mathfrak{c} \Theta \mathfrak{J}}$

$\mathfrak{b} * \mathfrak{b}$ in the context of Theorem 1.1

$\mathfrak{b} * \mathfrak{b}$ in the context of Theorem 1.2

$\|\cdot\|_{\mathbb{H}}^{2}$ in the context of Theorem 1.1

$\|\cdot\|_{\mathbb{H}}^{2}$ in the context of Theorem 1.2

$\mathbb{H}, \mathbb{L}$ in the context of Theorem 1.1

$\mathbb{H}, \mathbb{L}$ in the context of Theorem 1.2

$\mathfrak{L}_{\mathfrak{c}}$

$\mathfrak{J}_{0}$

$\mathfrak{L}_{I}$

$\partial_{A_{\gamma}}, \bar{\partial}_{A_{\gamma}}$

$\mathbb{V}_{0}, \mathbb{V}_{1}$

$\widehat{\vartheta}_{\gamma, r}$

$\Pi_{\zeta}$
Section 2.f

Equation (2-29)

Section 2.g

Part 2 of Section 4.a

Section 2.g

Part 2 of Section 4.a

Part 1 of Section 2.g

Part 2 of Section 2.g

Equation (3-1)

Step 1 of Section 3.a. See also

Equation (4-8).

Step 1 of Section 3.a

Step 1 of Section 5.a

Part 1 of Section 3.a.

Step 2 in Part 2 of Section 5.a

Step 2 of Section 3.a

Step 2 of Section 3.a

Step 2 of Section 3.a

Step 2 of Section 3.a

Step 2 of Section 3.a

Equation (3-3)

Step 4 of Section 3.a

Equations (3-5), (3-6) and the associated discussion

Equation (3-6)

Equation (6-1)

Equation (3-7)

Equation (6-2)

Step 1 of Section 3.b

Part 1 of Section 6.a

Equation (3-8)

Step 2 of Section 3.b

Step 2 of Section 3.b

Equation (3-12)

Step 3 of Section 3.b

Equation (3-15)

Step 4 of Section 3.b 
$\mathbb{L}_{\zeta}, \mathbb{H}_{\zeta}^{\perp}$

$\mathbb{T}$

$\mathbb{H}_{\gamma}, \mathbb{H} \frac{\perp}{\gamma *}, \mathbb{L}_{\gamma}, \mathbb{L}_{\gamma *}$

$\Pi_{\gamma *}$

$\mathfrak{L}_{\gamma}$

$\mathfrak{L}_{0}$

$\mathcal{B}$

$\mathcal{F}$

$\mathfrak{b}(\zeta), \mathfrak{b}_{* \zeta}, \mathfrak{e} \zeta$

$w$

$\gamma_{\mathcal{E}}$

$q_{\mathcal{E}}$

$z_{\mathcal{E}}$

$\operatorname{div}_{\mathcal{E}}$

$N$ : the normal bundle to $C$

$e_{C}$ : the exponential map defined on $N$

$\mathcal{D}_{C}$

$m_{\gamma^{-}}, m_{\gamma^{+}}$

$\varsigma_{q_{\mathcal{E}}}$

$\rho_{*}$

$\rho_{\Sigma}$

$R_{*}$

$R_{\mathcal{E}}$

$\mathcal{C}$

$\mathcal{E}$

$\mathcal{E}_{\gamma^{ \pm}}$

$\Xi_{\Sigma^{ \pm}}$

$\left(A^{*}, \psi^{*}\right)$

$N_{1}, N_{1 C}$

$\chi_{C}$

$\left(\mathbb{A}^{C, r}, \alpha^{C, r}\right)$

$\mathcal{E}_{k R}$

$U_{C}, U_{0}, U_{\gamma^{-}}, U_{\gamma^{+}}$

$3_{\Sigma \gamma^{ \pm}}$

$\left(\mathbb{A}^{C, r}, \alpha^{C, r}\right)$

$\alpha^{\gamma^{ \pm}}$

$\left(\mathbb{A}^{\gamma^{ \pm, r}}, \alpha^{\gamma^{ \pm, r}}\right)$
Step 4 of Section 3.b

Equation (3-17)

Step 5 of Section 3.b

Step 5 of Section 3.b

Step 5 of Section 3.b

Step 5 of Section 3.b

Section 3.e

Equation (3-36), Section 3.e

Lemma 3.10 in Section 3.f

Equation (4-1)

Part 1 of Section 4.a

Part 1 of Section 4.a

Equation (4-2)

Part 1 of Section 4.a

Part 2 of Section 4.a

Part 2 of Section 4.a

Equation (4-5)

Section 4.b

Equation (4-2), Constraint 2 in

Section 4.b

Equation (4-8)

Equation (4-8)

Equation (4-8)

Equation (4-8)

Introduction to Section 5.a

Introduction to Section 5.a

Introduction to Section 5.a

Section 5.a

Equations (5-2), (5-8) and (5-9)

Step 1 in Part 1 of Section 5.a

Step 1 in Part 1 of Section 5.a

Step 2 in Part 1 of Section 5.a

Part 2 of Section 5.a

Equation (5-3)

Step 1 in Part 2 of Section 5.b

Step 2 in Part 2 of Section 5.a

Equation (5-6)

Step 2 in Part 2 of Section 5.a 


$$
\begin{aligned}
& \chi_{\gamma^{ \pm}} \\
& \chi_{R \pm} \\
& \chi_{\mathcal{E}} \\
& \phi_{\gamma^{ \pm}} \\
& \xi_{C}, \xi_{\gamma^{ \pm}} \\
& \mathcal{K}, \mathcal{K}_{\text {smooth }}, \mathcal{K}_{*} \\
& \|\cdot\|_{\mathcal{K}} \\
& \|\cdot\|_{\infty},\|\cdot\|_{\mathcal{K}_{1}^{2}},\|\cdot\|_{\mathcal{K}} \\
& \left(\mathbb{A}^{C \xi r}, \alpha^{C \xi r}\right) \\
& \mathfrak{t}^{C \xi} \\
& \zeta_{*} \\
& \left(\mathbb{A}^{\gamma \xi \pm}, \alpha^{\gamma \xi \pm}\right) \\
& \left(\mathbb{A}^{\gamma \xi \pm, r}, \alpha^{\gamma \xi \pm, r}\right) \\
& \mathfrak{t}^{\gamma \xi \pm} \\
& \left(A^{\xi}, \psi^{\xi}\right) \\
& 3^{\xi}
\end{aligned}
$$$$
\mathfrak{Z}_{\mathcal{E}}
$$$$
\mathfrak{Z}_{k}
$$$$
I_{k}
$$$$
\phi_{k}
$$$$
\xi_{k}, \xi_{\Sigma k}
$$$$
\left(\nabla_{A^{\xi}}\right)_{S}
$$$$
\mathfrak{b}_{ \pm}
$$$$
\mathfrak{D}
$$$$
\mathbb{V}_{C 0}, \mathbb{V}_{C 1}
$$$$
K_{C_{\xi}}, K_{\gamma \xi \pm}
$$$$
\mathcal{K}^{2}
$$$$
\hat{\theta}_{C_{\xi}}, \hat{\theta}_{\gamma \xi+}
$$$$
t_{\xi}
$$$$
\Pi_{\xi}
$$$$
\mathbb{H}_{\xi}^{\perp}
$$$$
\mathbb{L} \frac{1}{\xi}
$$$$
\mathfrak{h}(\xi)
$$$$
\mathfrak{v}_{\mathfrak{h}}
$$$$
\chi_{ \pm}
$$$$
\mathfrak{b}_{0}
$$$$
\chi_{0}
$$$$
\mathfrak{v}_{\mathfrak{C}_{\xi}}
$$

Step 2 in Part 2 of Section 5.a

Step 2 in Part 2 of Section 5.a

Step 2 in Part 2 of Section 5.a

Step 3 of Section 5.b

Equation (5-12)

Step 4 in Section 5.b

Equation (5-13)

The paragraph following Equation (5-13)

Step 5 of Section 5.b

Step 5 of Section 5.b

Step 6 of Section 5.b

Step 6 of Section 5.b

Step 6 of Section 5.b

Step 6 of Section 5.b

Equation (5-15)

Equation (5-16)

Equation (5-17)

Section 5.c

Section 5.c

Section 5.c

Section 5.d

Equation (6-1)

Equation (6-2)

Equation (6-5)

Part 5 of Section 6.a

Part 6 of Section 6.a

Part 6 of Section 6.a

Equation (6-9)

Part 6 of Section 6.a

Part 6 of Section 6.a

Part 7 of Section 6.a

Section 6.d, Lemma 6.3

Equation (6-21)

Part 1 of Section 6.d

Part 2 of Section 6.d

Part 2 of Section 6.d

Equation (6-32) 


$\mathfrak{p}_{C}$
$\vartheta_{C, r}^{\dagger}$
$\mathfrak{b}_{C}$
$\mathfrak{v}_{\gamma \xi \pm}$
$\vartheta_{\gamma \xi^{-}, r}$
$\mathfrak{b}_{\gamma^{ \pm}}$
$\|\cdot\|_{*}$
$\mathcal{B}$
$\mathfrak{q}(\xi)$
$\mathfrak{m}_{\mathfrak{f}}(p, \rho)$
$\theta_{r}$
$\hat{\theta}$
$\mathcal{A}_{1}, \mathcal{A}_{2}$
$\mathcal{A}_{3}$
$\mathcal{A}_{4}$
$\xi_{\lambda}$
$\|\cdot\|_{\mathcal{L}}$
$\mathcal{L}$
$\mathcal{T}$
$\mathfrak{n}_{\mathfrak{f}}$
$\mathcal{T}_{0}$
$\mathcal{T}_{1}$
$\mathcal{T}_{2}$
$V_{0}$
$q$
$q^{*}$

Equation (6-33)

Equation (6-34)

Equation (6-36)

Equation (6-39)

Part 5 of Section 6.d

Part 5 of Section 6.d

Equation (6-51)

Proposition 6.4

Proposition 6.4

Equation (6-54)

Part 2 of Section 6.e

Part 3 of Section 6.e

Equation (6-67)

Equation (6-70)

Equation (6-74)

Proposition 7.1

Section 7.a

Section 7.a

Section 7.a

Equation (7-3)

Section 7.a, Part 1 of Section 7.e

Section 7.a, Part 2 of Section 7.e

Section 7.a, Part 3 of Section 7.e

Proposition 7.1, Section 7.f

Proposition 7.1, Section 7.f

Section 7.f

\section{References}

[1] A Jaffe, C Taubes, Vortices and monopoles. Structure of static gauge theories, Progress in Physics 2, Birkhäuser, Boston (1980) MR614447

[2] P Kronheimer, T Mrowka, Monopoles and three-manifolds, New Math. Monogr. 10, Cambridge Univ. Press (2007) MR2388043

[3] C B Morrey, Jr, Multiple integrals in the calculus of variations, Grund. der math. Wissenschaften 130, Springer, New York (1966) MR0202511

[4] CH Taubes, Arbitrary $N$-vortex solutions to the first order Ginzburg-Landau equations, Comm. Math. Phys. 72 (1980) 277-292 MR573986 
[5] CH Taubes, Seiberg-Witten and Gromov invariants for symplectic 4-manifolds, (R Wentworth, editor), First Int. Press Lecture Ser. 2, Int. Press, Somerville, MA (2000) MR1798809

[6] C H Taubes, The Seiberg-Witten equations and the Weinstein conjecture, Geom. Topol. 11 (2007) 2117-2202 MR2350473

[7] CH Taubes, The Seiberg-Witten equations and the Weinstein conjecture. II. More closed integral curves of the Reeb vector field, Geom. Topol. 13 (2009) 1337-1417 MR2496048

[8] C H Taubes, Embedded contact homology and Seiberg-Witten Floer cohomology I, Geom. Topol. 14 (2010) 2497-2581

[9] C H Taubes, Embedded contact homology and Seiberg-Witten Floer cohomology III, Geom. Topol. 14 (2010) 2721-2817

[10] C H Taubes, Embedded contact homology and Seiberg-Witten Floer cohomology IV, Geom. Topol. 14 (2010) 2819-2960

Department of Mathematics, Harvard University

Cambridge, MA 02138, USA

chtaubes@math. harvard.edu

Proposed: Rob Kirby

Seconded: Danny Calegari, Peter Ozsváth
Received: 15 November 2008

Revised: 11 May 2010 